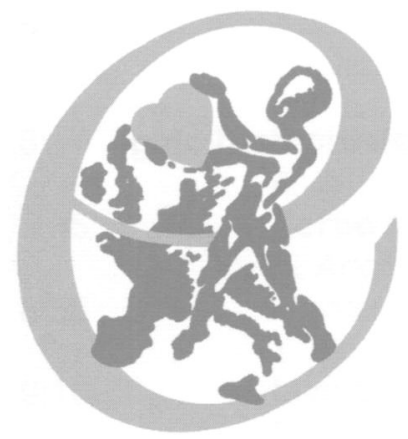

Volume 12 - Supplement 1 - Pages 1-87

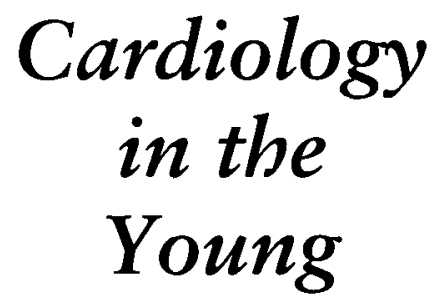

(C) 2002 Greenwich Medical Media Ltd

\title{
Abstracts and Posters of the XXXVII Annual Meeting of The Association for European Paediatric Cardiology, Porto, 15-18 May, 2002
}

\author{
Session 1: Imaging \\ 01 \\ Coronary flow reserve in patients after the Fontan \\ operation \\ A. Eicken, W. Sehening, Th. Genz, H. Kaemmerer, G. Balling, \\ S. Fratz, R. Lange, R. Blasini, R. Busch, J. Hess \\ German Heart Center .Munich, München, D
}

Introduction: Elevated coronary sinus pressure may contribute to late post-operative cardiac failure after a Fontan Operation. The current study was designed to investigate the impact of coronary sinus drainage on coronary flow reserve (CFR) and myocardial function in patients after a modified Fontan operation.

Methods: 36 unselected patients (age 17.6 6.5 y) with univentricular circulation (DILV $=11, \mathrm{TA}=12$, other complex cardiac lesions $=13$ ) 9.74 .2 after a Fontan operation (RA-RV anastomosis $=9$, RA-PA anastomosis $=17, \mathrm{TCPC}=10$ ) were examined. Cardiac catheterization was performed under general anesthesia. Coronary flow reserve (FloWire, Cardiometrics, Inc.) was determined after intracoronary application of papaverine.

Results: Measurement of CFR in the right coronary artery (RCA) was possible in $26 / 36$ and in the left coronary artery (LCA) in 32/36 patients. Mean CFR-RCA was 3.951 .2 and mean CFR-LCA was 4.2 1.6. Coronary sinus drainage into the systemic - or pulmonary venous compartment did not significantly affect CFR. $41 \%$ CFR-values were $<3.5$. There was a significant correlation between CFR-RCA and pulmonary arteriolar resistance $(p<0.05)$. Systolic ventricular function was significantly higher in patients with a morphologic left ventricle $(\mathrm{p}=0.02)$ but was not correlated with CFR-values. "Coronary arterial fistulas" were seen in $33 \%$ of the patients.

Conclusion: Coronary flow reserve is frequently reduced in patients after a Fontan operation. Site of coronary drainage into the "high pressure" systemic atrium or the "low pressure" pulmonary venous atrium did not significantly affect CFR. Elevated coronary sinus pressure may lead to increased Thebesian venous How: This may be an explanation for the high incidence of "coronary arterial fistulas". Explanation of the correlation between CFR and pulmonary resistance demands further evaluation.
02

Myocardial perfusion and coronary flow reserve assessed by positron emission tomography in patients after Fontan-like operations M. Hauser, A. Khn, F.M. Bengel, U. Sauer, S. G. Nekolla, M. Schwaiger, J. Hess

German Heart Centre Munich, Dept. of Paediatric Cardiology, Munich, $D$

Background: Ventricular dysfunction frequently observed in patients after Fontan-like operations (FLO) is a serious complication that might contribute to poor long-term results. Ischemic heart disease will have debilitating consequences on a Fontan heart. Aim of the clinical investigation was the assessment of myocardial perfusion correlated to clinical and hemodynamic parameters in patients after FLO.

Methods: 10 patients (15.8 SD 5.01 years) after FLO had transoesophageal echocardiography and cardiac catheterization 9.3 (SD 4.2) years after surgery. Myocardial perfusion was assessed by NH3-PET at rest and after vasodilatation with adenosine; the results were compared with 10 healthy adults ( 26.1 SD 6.3 years). Results: Ventricular function was normal in 4, and reduced in 6 patients; endsystolic and enddiastolic meridional wall stress was significantly elevated in the FLO group. Selective coronary angiography reveals no stenosis of the subepicardial coronary arteries. Compared to normals, myocardial blood flow (MBF) at rest was significantly higher in the FLO $(0.99$ SD 0.25 vs. 0.77 SD $0.17 \mathrm{ml} / \mathrm{g} / \mathrm{min} . \mathrm{p}<0.026$ ), whereas $\mathrm{MBF}$ after maximal vasodjlatation (2.12 SD 0.78 vs. $3.10 \mathrm{SD} 0.85 \mathrm{ml} / \mathrm{g} / \mathrm{min} . \mathrm{p}<0.023)$ and coronary flow reserve (CFR) was significantly reduced (2.5 SD 0.88 vs. $4.1 \mathrm{SD} 1.01 . \mathrm{p}<0.0035)$, especially in those with impaired ventricular function; coronary vascular resistance after vasodilatation was significantly elevated in the FLO (44.0 SD 18.3 vs. $28.3 \mathrm{SD}$ $8.45 \mathrm{mmHg} / \mathrm{ml} / \mathrm{g} / \mathrm{min} . \mathrm{p}<0.007$ ).

Conclusion: Altered MBF, increased meridional wall stress and impaired CFR are common findings in FLO; attenuated CFR and reduced ventricular function are significantly correlated and may be important risk factors for the long-term outcome. 
03

Safety and reliability of Dobutamine-stress echocardiography for detection of myocardial ischemia in children

S. Di Filippo, M.J. Raboisson, B. Semiond, F. Sassolas, A. Bozio Pediatric Cardiology, Hopital Cardiologique Louis Pradel, Lyon, F

Detection of myocardial ischemia is required in children with congenital, acquired or postoperative coronary artery diseases. The aims of this prospective study were to determine the safety and feasability of Dobutamine-stress echocardiography (DSE) in children, and to assess its reliability for detection of myocardial ischemia and coronary disease.

Methods: DSE was performed according to the standard protocol used in adults. Selective coronary angiography and/or Thallium scintigraphy were performed for comparison. Seventy-five DSEs were performed in 71 patients ( 31 females, 40 males, median age $=11$ years). Cardiac pathologies were: posttransplant monitoring (40 cases), postSwitch operation monitoring (13), abnormal origin of the left coronary artery from the pulmonary trunk (6), Kawasaki aneurisms (6), coronary fistulae (5), left ventricle aneurism (1) and miscellaneous (4). Sixty-two were performed routinely and 13 were motivated by: chest pain (8), dyspnea (2), left ventricle dysfunction (2) or syncope (1). Fourteen cases were receiving antihypertensive medication but none with beta adrenergic antagonistic therapy.

Results: The maximum dose stage of $40 \mathrm{mcg} / \mathrm{kg} / \mathrm{mn}$ was reached in $72 / 75$ cases ( $96 \%$ ) and maximum dose was $30 \mathrm{mcg} / \mathrm{kg} / \mathrm{mm}$ in 3 (chest pain). The most frequent clinical side effect was palpitations (15\%). Maximum heart rate was 90 to $183 \mathrm{bpm}$ ( 30 to $351 \%$ of basal value and 43 to $90 \%$ of theoretical maximum). Maximum blood pressure was 100 to $194 \mathrm{mmHg}$ ( 7 to $109 \%$ of basal value). No sustained arrhythmia, nor significant ST segment change, occured. Twenty (27\%) DSEs were abnormal: 8 posttransplant, 2 Kawasaki, 4 postSwitch, 4 non-operated coronary origin from pulmonary trunk, 1 non-operated coronary fistulae and 1 left ventricle aneurism (15 of them were clinically asymptomatic). DSE sensitivity and specificity were respectively $100 \%$ and $76 \%$ when compared to Thallium scintigraphy (31 cases) and 70\% and $90 \%$ when compared to coronary angiography (40 cases).

Conclusion: DSE is probably the best method of monitoring coronary artery disease in children, combining both reasonable sensitivity and specificity without being invasive, requiring hospitalization or engendering significant morbidity.

\section{4}

The Quebec Child Telehealth Network: implementation and early results

M.J. Bland, A. Cloutier, N. van Doesburg, M. Bellavance

The Montréal Children's Hospital, Montréal, CDN

We present the successful implementation of the Quebec Child Telehealth Network (QCTN) and early results of its first application, telediagnosis in paediatric cardiology.

The QCTN was founded in 1997 by representatives of the 4 university hospitals (UHs) that offer paediatric cardiology services in the province of Quebec, Canada. In addition to the $4 \mathrm{UHs}, 32$ regional centres ( $\mathrm{RCs}$ ) were chosen to participate in the QCTN using the following criteria: locally available resources, local population size and birth rate, distance from a UH, and previously established outreach or satellite clinics. In 1998-9, the 36 QCTN participants were equipped with a Codec device (Cifra Medical SDX-300) and a small camera that allows two-way viewing during transmissions. With this equipment, real-time echocardiographic images were relayed to the
UHs at distances varying from 20 to $1500 \mathrm{~km}$ within Quebec at a bandwidth of 448 or $512 \mathrm{kbps}$ through ATM or ISDN lines. An Internet protocol in $\mathrm{H} 323$ mode was used for these transmissions on the provincial healthcare telecommunications network.

In the year 2000, 114 transmissions were performed: 92 (81\%) for new patients, 22 (19\%) for follow-up. The most active RCs were those located more than $100 \mathrm{~km}$ from the nearest UH $(77 \%$ of transmissions) and those with established satellite paediatric cardiology clinics or a previous referral pattern to a UH $(67 \%$ of transmissions). Telediagnosis avoided immediate transfers in $33 / 114$ $(29 \%)$ and elective visits in $57 / 114(50 \%)$. It confirmed cardiac problems treatable in the RC in 11/114 (10\%) and identified congenital heart defects requiring transfer in $7 / 114(6 \%)$. Studies were judged adequate in all but 2 cases $(2 \%)$. Four children $(4 \%)$ required transfer for other paediatric problems.

In conclusion, the QCTN demonstrates the importance of remoteness from UHs as well as previously established outreach programs or teferral patterns for successful implementation of the network. Our results thus far confirm the clinical and practical value of telediagnosis in pacdiatric cardiology.

\section{5}

Superior quantification of pulmonary blood flow by phase velocity magnetic resonance imaging compared to lung perfusion scintigraphy in patients with Fontan like circulation

H.C. Stem, S. Fratz, M. Schuaiger, J. Hess

Dewtsches Herzzentrum München, München, D

Quantitative evaluation of right/left lung flow ratio in Fontan like palliation is of particular interest. Although lung perfusion scintigraphy is considered to be the golden standard, lung flow ratio is difficult to measure because of preferential drainage of caval flow into either lung side.

We evaluated the feasibility and accuracy of phase velocity cine (PVC) magnetic resonance imaging (MRI) to determine the ratio of right to left pulmonary perfusion compared to lung perfusion scintigraphy.

Methods: The study group comprised 15 patients (age 167.4 yrs.) after atriopulmonary $(\mathrm{n}=9)$ or cavopulmonary $(\mathrm{n}=6)$ connections. Right and left pulmonary blood flow was quantified with PVC-MRI. Right to left flow ratio was also rated by standard lung perfusion $99 \mathrm{mTc}$ scintigraphy. Patients after TCPC palliation recieved on two subsequent days an injection into the upper and lower limb. The right lung flow quota of the two injections were added and set into proportion to the two totalled left lung flow quotas.

12 patients (age: $158 \mathrm{yrs}$.) with pulmonary perfusion abnormalities and biventricular circulation served as controls. They underwent both lung perfusion scintigraphy and MRI flow studies. This group was selected for control, as complete mixing of the radiotracer in the subpulmonary ventricle could be suspected.

Results: In patients with Fontan like circulation there was considerable difference in right pulmonary blood flow as measured by scintigraphy and PVC-MRI. Bland-Altman analysis showed a difference of $7.110 .1 \%$ right pulmonary blood flow in the study group. Upper and lower limits of agreement were $27.2 \%$ and $-13.0 \%$, respectively, and therefore clinically unacceptable.

In control patients, however, correlation between pulmonary blood flow by perfusion scintigraphy and MRI was excellent. There was a negligible difference of $-1.62 .8 \%$ between the two methods. Limits of agreement were $4.0 \%$ and $-7.2 \%$, respectively (Figure 1 ). Conclusion: In biventricular circulation, data of right/left lung flow ratio correlate well, when assessed by lung perfusion scintigraphy 


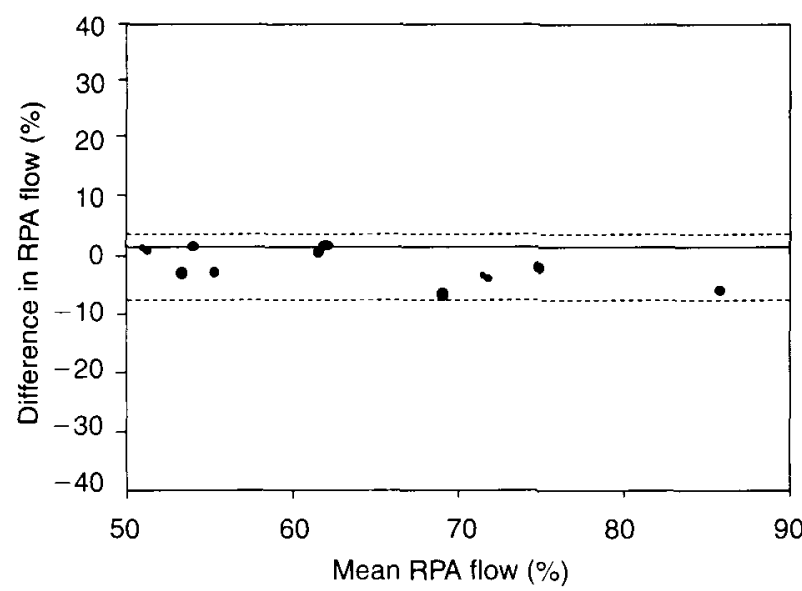

and PVC-MRI. In patients with Fontan like circulation, MRI seems to be superior for quantification of pulmonary blood flow. In these patients lung scintigraphy reveals a considerable error due to preferential caval flow in either lung side depending on the injection site.

\section{6}

Which tissue Doppler echocardiography data are useful in the early diagnosis of hypertrophic cardiomyopathy in children with a familial pre-disposition?

L.B. Pauliks, .M. Vugel, S. Dickie, W. McKema, J.E. Deanfield

Lniversity of Colorado - The Children's Hospital Denver, Denver, USA

In adults with hypertrophic cardiomyopathy (HCM), systolic myocardial velocities at the base of the heart are decreased but little is known about regional function or the pattern in children with HCM. We examined 22 children aged $8.34(0.1-15.9)$ years with a family history of $\mathrm{HCM}(\mathrm{FH})$. Controls were 121 agematched children with normal hearts and 23 patients with LV hypertrophy (LVH) of other aetiology (10 aortic coarctation, 13 aortic stenosis). Tissue Doppler of the LV free wall was performed with a frame rate $>98$ Hertz (GE Vingmed System V). Systolic and diastolic velocities were measured at LV base, middle and apex. The displacement of the myocardiun from base to apex was quantified in a contimuous fashion by integrating the velocity data (tissue tracking). Tissue Doppler data were evaluated blindly without knowledge of clinical or ECG data.

Results: In all normal children and controls with LVH, the highest velocities and the largest displacement were found at the base with a gradual reduction of velocities and displacement towards the apex. In all segments, systolic respectively diastolic velocities were uniformly directed. In the FH group, 11/22 patients had wall motion abnormalities. In $4 \mathrm{FH}$ children, apical velocities were inverted. Moreover, $7 \mathrm{FH}$ children did not show the pattern of gradual decrease from base to apex so that their peak velocities and displacement occurred in the middle segment of the LV rather than at the base. Retrospective correlation with clinical data revealed that $13 / 22$ children with $\mathrm{FH}$ fulfilled WHO criteria of HCM. Wall motion abnormalities were present in $10 / 13$ children with WHO criteria for HCM (77\%) but only $1 / 9$ without HCM $(p<0.0001)$. All children who fulfilled WHO criteria had reduced systolic and diastolic velocities at the base. Yet, in $7 / 13$ patients the lower velocities at the base were at least partly due to a shift of peak velocities and maximum displacement towards the apex. Thus, the presence of an abnormal regional distribution of velocities and displacement was more specific for HCM than reduced peak velocities alone; it may improve early diagnosis and was helpful in differentiating HCM from other forms of LVH.
07

Early results and echocardiographic evaluation of bovine jugular vein (contegra) used for right ventricular outflow tract reconstruction H.K. Najm, M. Almotairi, J. Du Plessis, A. Omran, M. Godman King Abdulaziz Cariac Center, Riyadh, SAU

Objective: Many of operations to correct congenital heart defects require the insertion of a valved conduit between the right ventricle and the pulmonary artery. Currently used conduits are frequently associated with development of stenosis or incompetence of the valve. In the search for the "perfect" conduit, bovine jugular vein has recently been introduced. This valve is a totally integrated valve conduit available in the shelf with sizes 12 to $22 \mathrm{~mm}$. Other advantages include the length of the conduit and resilience.

Patients and method: Twenty children underwent reconstruction of the right ventricular outflow tract using bovine jugular vein at a median age of 8.2 months. Two in the orthotopic position (Ross procedures), and 18 in a variety of congenital cardiac lesions. Seven underwent previous surgical procedures. The duration of cardiopulmonary bypass was a mean of 14646 and a myocardial ischemic time of 7038. Mean conduit diameter was 14.53 (range $12-22 \mathrm{~mm}$ ). All children were evaluated by echocardiography immediately after repair and at 1,3,6,12 months postoperatively. Results: There was one early and one late mortality neither related to conduit replacement. Unique echocardiographic features of this conduit include: 1 . Flow reversal in the conduit with no correlation with significant pulmonary regurgitation. 2 . Reduced or absent valve leaflet motion identified in the pre-discharge echo in some patients. 3 . Improved leaflet motion at later follow-up.

Conclusions: This is a promising conduit. In addition to the versatility of this conduit it performs satisfactorily in the right ventricular outflow tract. Flow reversal in the outflow part of the conduit may reflect the dispensability feature of this conduit. Reduce leaflet motion does not correlate with significant pulmonary regurgitation and will improve after discharge.

08

Relationship between myocardial fibrosis and electrical instability in patients with hypertrophic cardiomyopathy G. Limongelli, G. Pacileo, P. Calabr, F. Cerrato, S. Severino, G. Di Salvo, R. Calabro

Pediatric Cardiology - Monaldi Hospital, Naples, I

Objective: Life threatening ventricular arrhythmias and interstitial myocardial fibrosis represent risk factors for sudden death in patients with hypertrophic cardiomyopathy (HCM). The aim of this study is to evaluate a relationship between interstitial fibrosis and myocardial electrical instability in patients with HCM.

Methods: We studied 48 young subjects: 22 pts (mean age 2113 yrs, $12 \mathrm{M}, 10 \mathrm{~F}, \mathrm{BSA} 1.60 .5$ ) (HCM Group) and 26 age- and BSA matched healty subjects (Control Group). Subjects underwent an echocardiographic examination with myocardial integrated backscatter (MIB) analysis for ultrasonic tissue characterization of myocardial fibrosis. The calibrated myocardial intensity was obtained measuring myocardial reflectivity from the interventricular septum (IVSRf) and from left ventricular posterior wall (PWRf), and measuring the difference of each parameter with pericardium reflectivity (IVSRf-PerRf, PWRf-PerRf). Twenty-four hours Holter monitoring was recorded to evaluate ventricular arrhythmias. Ventricular late potentials (VLP) (QRSD, LAS40, RMS40) were assessed by Signal Averaged ECG (SAECG).

Results: Compared to the control group, the HCM group showed both in the IVS and PW increased myocardial intensity (-2910 
vs. $-354 \mathrm{P}=0.007$ and 298 vs. $335 \mathrm{P}<0.05$ respectively) both at IVS and PW; 5 HCM patients and no controls showed presence of VLP; 4 patients had Lown $4 \mathrm{~b}$ (all with presence of VLP). At regression logistic analysis, patients with ventricular arrhythmias Lown class $4 \mathrm{~b}$ had higher values of myocardial intensity at IVS (IVSRf, $p=0.04$, IC $1.1-6.82, \quad$ OR $=1.27$; IVSRf-PerRf, $p=0.042, \mathrm{IC}=1,006-1,42, \mathrm{OR}=1,19)$ and presence of VLP $(\mathrm{p}=0.026, \mathrm{IC}=0.04-0.82, \mathrm{OR}=3.67)$, independently to age, gender, body surface area and wall thickness.

Conclusions: In HCM group, myocardial reflectivity is significantly higher than in normal subjects (increase of myocardial fibrosis); ventricular arrhythmias are associated with presence of VLP and highest values of myocardial reflectivity, suggesting a potential role of interstitial fibrosis as determinant of electrical non-homogeneity and ventricular arrhythmic events.

\section{9}

Dynamic three-dimensional (3D) colour doppler ultrasound of human fetal intracardiac flow - potential for dynamic diagnosis

J. Deng, R. Yátes, I.D. Sullivan, D. McDonald, A.D. Linney,

W.R. Lees, R.H. Anderson, C.H. Rodeck

University College London, London, UK

Objectives: To develop a ultrasound technique for dynamic 3D (4D) imaging of fetal cardiac flow and for improving prenatal detection of complex malformations.

Methods: Grey-scale and colour velocity Doppler echocardiography was performed on 12 fetuses to provide serial anatomical and rheologic tomograms which were registered in 3D. Additional spectral Doppler ultrasound was performed simultaneously to record umbilical arterial waveforms, thus providing the cardiac cyclical information to facilitate motion artefact removal.

Results: Acquisitions were successful in 8 of 15 attempts. Useful 4D images of the cardiac flow was obtained in 6 of the 8 datasets. In one case with double outlet right ventricle diagnosed on crosssectional images, $4 \mathrm{D}$ views throughout the cardiac cycle revealed the aortic overriding only seen in systole (black arrow) with the narrowed pulmonary trunk. The overall diagnosis is tetralogy of Fallot with associated DORV.

Discussion: The technique has made possible the prenatal visualisation of the spatial distribution and direction of intracardiac flow in 4D. It suggests that diagnosis of malformations can be made on the basis of morphological and haemodynamic changes throughout the cardiac cycle, offering significant additional information to conventional methods.

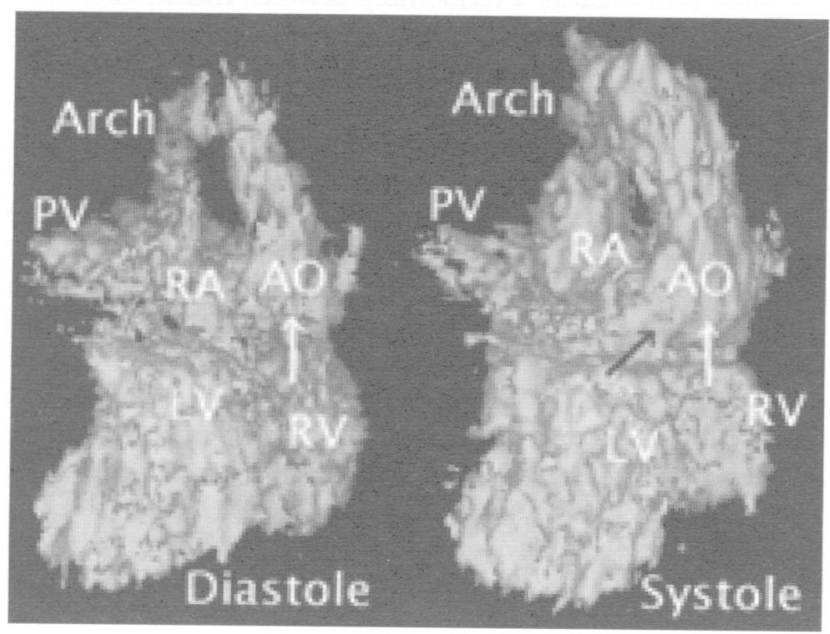

Conclusion: Dynamic three-dimensional ultrasound imaging of the fetal cardiac flow is not only possible but also provides unique information to improve diagnostic precision in complex cardiac malformations and a new means for modelling cardiovasculature and haemodynamics and their interactions.

\section{Session 2: Surgery}

10

Pulmonary artery banding with early and late telemetric adjustment

A.F. Corno, N. Sekarki, M.A. Bernath, M. Payot, P. Tozzi,

L.K. von Segesser

Centre Hospitalier Universitaire Vaudois, Lausanne, CH

Objective. Control of pulmonary blood flow is difficult in palliative procedures for complex congenital heart defects.

A new wireless, battery free, telemetrically controlled, implantable device (FloWatch, EndoArt S.A., Lausanne, Switzerland) allowing for progressive occlusion/reopening of the device through a remote control at the wanted percentage of occlusion (adjustable pulmonary artery banding) had experimental evaluation.

Methods. Eleven Gttingen mini-pigs underwent FloWatch implantation around the main pulmonary artery through left thoracotomy. The first Group $(\mathrm{n}=4)$, mean age 18.20.1 weeks, mean body weight $12.00 .1 \mathrm{~kg}$, underwent FloWatch implantation as device tolerance test.

The second Group $(n=7)$, mean age 8.63 .4 weeks, mean body weight $5.01 .4 \mathrm{~kg}$, underwent functional evaluation: at implantation, $1,3,5,8$ and 10 weeks after implantation, the device was progressively occluded and reopened, with Doppler evaluation of the developed pressure gradient.

Results. At mean interval of 24 weeks after implantation the 4 mini-pigs of first Group were sacrificed (mean age of 42.30 .1 weeks, mean body weight $25.13 .2 \mathrm{~kg}$ ); the device was well functioning and histology revealed minor inflammatory reaction to the device.

The possibility of narrowing/releasing the pulmonary artery was demonstrated in all 7 mini-pigs of second Group at implantation and during follow-up: at last control their mean age was 17.53.7 weeks and the body weight $9.33 .8 \mathrm{~kg}$.

The Table shows the results of all Doppler evaluations (mean SD) of the correlation of 2 nd degree polynomial fit between $\%$ occlusion and Doppler gradient (A) and the Width of FloWatch regulation (Maximum value minus minimum value of Doppler gradient in $\mathrm{mmHg}$ ) (B) and the Figure shows a single example. Conclusion. Complete control of pulmonary blood flow is possible with a new implantable device allowing for pulmonary artery banding with early and late telemetric flow adjustment.

11

Results of surgical repair in patients suitable for primary angioplasty of coarctation of the aorta J.D.R. Thomson, R. Guerrero, C. Van Doorn, J.L. Gibbs, K. G. Watterson Yorkshire heart centre, Leeds, UK

Aims: To assess the results of surgical repair of coarctation in patients greater than 3 months of age with lesions suitable for primary angioplasty.

Patients and methods: Retropective identification of 50 consecutive surgically repaired patients (single surgeon) greater than 3 months of age over a 10 year period $(1 / 1990-2 / 2000)$. All patients had discrete lesions that appeared suitable for primary angioplasty. Mode of repair was patch augmentation $(n=2)$ and extended end to end repair $(\mathrm{n}=48)$. Age and weight at operation was 3.1 
nonths-17.5 years (median 30.2 months) and $3.1-58 \mathrm{~kg}$ median 14) respectively. End points were death, paralysis and ecoarctation. Patients were prospectively assessed for residual or ecoarctation (defined as either a $25 \mathrm{mmHg}$ pressure gradient setween upper and lower limb by blood pressure or Doppler :chocardiography) by a single operator evaluating femoral pulses, upper and lower limb blood pressure and ascending/descending Joppler aortic velocities.

Results: Median time to follow up was 5.1 years $(0.5-10.5)$. There vere no deaths and no neurological injuries. Post operative compliations occurred in $4 / 50$ patients $(8 \%, 95 \%$ CI $0.2-7.8)$ (1 chyothorax, 1 SVT, 1 pneumonia, 1 pneumothorax). All complications esolved without long term consequences. No patient was found to lave residual or recoarctation at prospective follow-up (48/50 $96 \%$ ) of patients were prospectively assessed, data on 2 patients refusal to attend) was taken from the most recent outpatient visit). Conclusion: $100 \%$ of patients with discrete coarctation repaired at over 3 months of age remained alive, neurologically intact and without residual or restenosis at up to 10 years follow-up. Post sperative complications were all minor and self limiting. Surgical epair in the modern era produces superior long term results to salloon angioplasty and should be considered the treatment of :hoice in discrete coarctation.

12 Staged surgical approach of neonates with iniventricular heart, transposition of the great irteries and sub-aortic obstruction

W. Daenen, B. Eyskens, D. Boshoff, L. Mertens, F. Marchau,

M. Geurillig, B. Meyns

University Hospital "Gasthuisberg", Leuven, B

Background: The surgical approach of neonates with Univenricular Heart (UVH), TGA and excessive pulmonary blood-flow remains a challenge. A Norwood operation has been used in this condition with a considerable surgical and neurological risk.

PA banding (PAB) remains a valuable option, but may induce ventricular hypertrophy, restriction of the bulboventricular foramen (especially in cases with coarctation of the aorta) and dysplastic changes of the pulmonary valve. These secondary changes might compromise a later Damus-Kaye-Stansel (DKS) operation and/or a subsequent Fontan repair. Our experience with this approach is the subject of this paper.

Methods: 25 Neonates underwent PAB for UVH, TGA and pulmonary hypertension between 11/1994 and 12/2001. Coarctation repair was associated in 14 patients.

The second stage operation was a bidrectional Glenn in $1 \mathrm{pt}$ and a DKS connection in 18 pts after a median interval of 9.0 months (range 1-108 months). The lenght of this interval was dictated by one or more of the following factors: degree of ventricular hypertrophy, restriction of the bulboventricular foramen and/or increased PA pressure. The DKS connection was constructed without any foreign material using resorbable sutures in the last 14 pts. Associated procedures were: bidirectional Glenn/hemiFontan (14 pts), aortopulmonary shunt (2 pts), homograft reconstruction RVOT (1 pt) and Fontan repair (1pt). 6 pts are waiting for the second stage operation. Ten patients underwent (after a mean interval of $2.7+0.6$ years) a Fontan operation at a third stage. 7 pts are suitable candidates for a Fontan repair.

Results: There was no mortality neither early nor late. The mean follow-up was $3.3+2$ years after the DKS operation and $1.9+1$ years after the Fontan repair. Echocardiography revealed a mean pulmonary regurgitation score of $0.8+0.6$ (on a four grade scale). All patients - except 3 pts with a mild gradient
$<10 \mathrm{mmHg}-$ had laminar flow in the ascending aorta. All Fontan repairs did well. 7 out of 9 fenestrations were closed by catheter device.

Conclusions:

1) A neonatal PAB followed by a DKS connection does not compromise a subsequent Fontan repair.

2) This approach carries a low surgical risk; the clinical and neurological outcome is excellent.

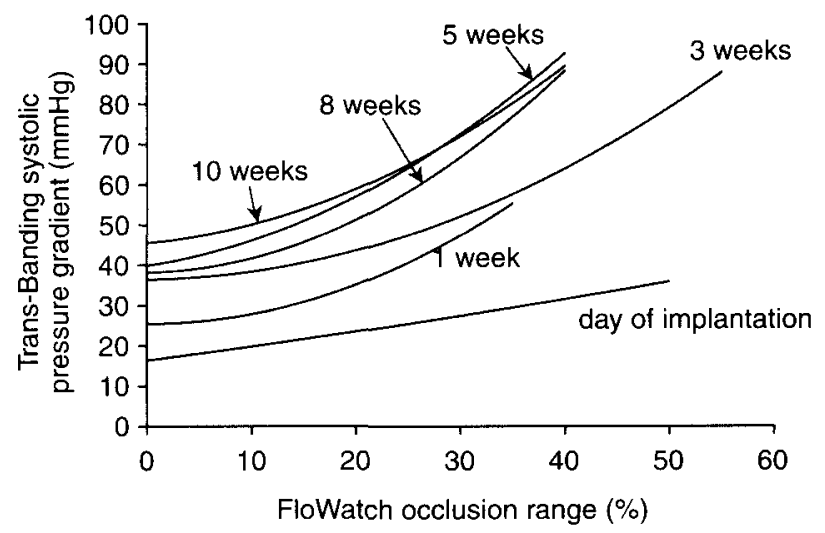

13

Percutaneous versus surgical closure of ostium secundum atrial defect

G. Butera, M. Chessa, R. Youssef, M.R. Bini, A. Giamberti, G. Pome, $M$. Drago, L. Rosti, D. Negura, A. Frigiola, M. Carminati.

Istituto Policlinico San Donato, S. Donato Milanese (MI), I

Background: Surgical atrial septal defect (ASD) closure provides excellent results. With the current rise of percutaneous techniques, a comparison is needed.

Aim: To compare percutaneous and surgical closure of ASD

Patients and methods: Between December 1988 and August 2001, 919 patients were referred to our institution with a diagnosis of secundum ASD. Four hundred and forty consecutive pts underwent percutaneous ASD closure (Group A). Two devices were used: Amplatzer Septal Occluder in 273 pts (62\%) and CardioSEAL/StarFLEX in 167 pts (38\%). Four hundred and ninety consecutive pts underwent ostium secundum ASD surgical repair (Group B). There was a slight difference in mean age between the two groups (Group A: 25.619 yrs vs. Group B: 22.517 .5 yrs; $\mathrm{p}=0.05$ ).

Results: Hospital stay was lower in Group A (3.30.6 days vs. 10.12.6 days; $p<0.0001)$. Total number of complications was higher in Group B (36\% vs. $6 \% ; \mathrm{p}<0.0001)$. In Group A major complications included: (a) device embolisation needing surgical retrieval (11 pts; $2.5 \%$ ); (b) surgical repair of femoral vessels (2 pts; $0.4 \%) ;(\mathrm{c})$ arrhythmias ( 9 pts; $2 \%$ ); (d) trombus formation on the device ( 2 pts; $0.4 \%$ ); (e) others ( 4 pts; $0.8 \%$ ). In Group B complications included: minor transient complications in $23 \%$ of the pts (arrhythmias, respiratory infections, pericardial effusion, anemia, pneumothorax,others); severe transient in $4 \%$ (arrhytmias, severe bleeding, heart failure, transient complete AV block, cardiac tamponade); severe with sequelae in $0.75 \%$ (neurologic, complete AV Block needing PM implantation). Transfusion rate was 3\%. Surgical drainage for pericardial or pleural effusion or for pneumothorax was needed in $4.5 \%$. Chest reopening for severe bleeding was in $0.5 \%$. Re-operation for patch detachement occurred in 2 pts. No early deaths occurred in both groups. Residual shunt at discharge was trivial and there were no differences between the two groups. Five pts of Group A and 5 pts of Group B were treated for a significant residual shunt after surgical ASD closure. 
Conclusions: Percutaneous ASD closure provides excellent results, with lower complication, no sequelae and shorter hospital stay.

14

Use of bovine jugular vein conduit to reconstruct the

right ventricular outflow tract: early results

Y. Boudjemline, D. Bonnet, T. Abdel Massih, G. Agnoletti, F. Jaubert, D. Sidi, P. Vouh

Cardiologie Pdiatrique, Hopital Necker-Enfants-Malades, Paris, $F$

Objective: We evaluate early results of bovine jugular vein valved conduits in the pulmonary outflow.

Methods: Between April 2000 and September 2001, 31 conduits were placed in the outflow of the right ventricle. Patients who received a conduit as a staged surgical procedure were excluded from the present study $(n=3)$. Diagnosis included truncus arteriosus ( $\mathrm{n}=9$ patients), tetralogy of Fallot without and with pulmonary atresia with ventricular septal defect (respectively $n=5$ and 6 patients), aortic valve stenosis ( $n=4$ patients), complex transposition of great arteries ( $n=2$ patients), and absent pulmonary valve syndrome ( $\mathrm{n}=2$ patients). Implantation age ranged from 0 to 21 years (mean 3.4). Conduit diameter ranged from 12 to $20-\mathrm{mm}$ (median 14). Transthoracic echocardiographic and Doppler evaluations were performed at discharge and $\mathbf{3}$ months after surgery. Patients with significant pulmonary regurgitation and/or stenosis underwent cardiac catheterization for precise evaluation.

Results: Four patients died during the follow-up period. Three deaths were unrelated to the conduit. One death was related to the complete thrombosis of the conduit. At the 3 months evaluation, pulmonary valve regurgitation was absent or trivial in 19 , mild in 2 and severe in 3 of 24 survivors. Four patients had non-fatal conduit-related complications. A transient thrombus formation within one leaflet was noted postoperatively in a patient with a moderate pulmonary regurgitation. Three patients required reoperation 3 to 5.8 months after the implantation for conduit failure (mean 4.3). Cardiac catheterization before replacement revealed an anevrysmal dilation of the conduit below a severe stenosis of the pulmonary bifurcation due to important neointimal proliferation. Conclusions: Bovine jugular vein valved conduits function well 3 months after their insertion. However, early failure can occur because of exaggerated intimal proliferation or thrombotic process within the conduit. In regard of these complications, close echocardiographic follow-up is warranted during the first weeks after implantation.

15

The impact of ventricular morphology on outcome following completion total cavopulmonary connection S.P. McGuirk, S.M. Langley, O. Stumper, R. Dhillon, P. Miller, J.V. de Giovanni, J.G. Wright, W.J. Brawn, D.J. Barron

Birmingham Children's Hospital, Birmingham, UK

Objective: To determine the outcome of patients undergoing completion total cavopulmonary connection (TCPC) with either a morphological left or right ventricle in the systemic circulation. Method: Between August 1996 and September 2001, completion TCPC was performed in 105 patients, 59 with a hypoplastic left ventricle ( $R V$ group), and 44 with a hypoplastic right ventricle (LV group). Two with indeterminate morphology were excluded. Fifty-eight patients underwent primary Norwood palliation (RV group $n=46, L V$ group $n=12$ ). Follow-up was complete, median 17 months. Total follow-up was 190 patient years.

Results: There were 2 early deaths (1.9\%), one in each group $(\mathrm{p}=0.85)$. Mean post-operative pulmonary artery pressure
$( \pm 1 \mathrm{SD})$ was $14.2 \pm 2.0 \mathrm{mmHg}$ in the $\mathrm{RV}$ group verses $13.5 \pm 2.0 \mathrm{mmHg}$ in the $\mathrm{LV}$ group $(\mathrm{p}=0.13)$. Median inhospital stay was 19 days (RV group) vs. 16 days (LV group), $\mathrm{p}=0.06$. Four required early reintervention, 3 in the RV group and 1 in the LV group $(p=0.46)$. Five year freedom from reoperation was $92.7 \pm 3.6 \%$ in the RV group and $97.6 \pm 2.3 \%$ in the $L V$ group $(p=0.23$ ). Five year survival was $96.0 \pm 2.8 \%$ in the RV group and $97.6 \pm 2.4 \%$ in the $L V$ group $(p=0.79)$. All survivors are in NYHA class I or II.

Conclusion: Despite concerns regarding outcome of patients with a right systemic ventricle in the fontan circuit, this study has demonstrated low early mortality, low post-operative pulmonary artery pressures, high freedom from re-operation and excellent functional outcome and survival independent of the morphology of the systemic ventricle.

16

The spectrum of native cardiac disease requiring transplantation in pediatric age

D. Mancuso, A. Angelini, C. Frescura, F. Calabrese, O. Milanesi,

A.P. Caforio, P.A. Pellegrino, D. Casarotto, G. Thiene

Dept of Pathology, Padua, I

Cardiac transplantation represents an accepted treatment for infants and children ( $<18$ years old) with congenital and acquired end-stage heart disease. Between March 1987 and December 2000, 37 patients (24 males and 13 females), aged 38 days to 18 years, underwent orthotopic cardiac transplantation at the University of Padua.

Hearts removed at transplantation were investigated through macroscopy, histology, immunohistochemistry and, when indicated, ultrastructural and molecular studies in order to assess the pathologic substrates of end stage cardiac failure and their relevance to the outcome of the operation. They were divided into 4 groups: a) Congenital heart disease ( 9 cases, $24 \%$ ), 7 of them with previous surgery: double inlet ventricle (2), Ebstein's disease (2), non compacted ("spongy") myocardium (2), pulmonary atresia (1), hypoplastic left heart syndrome (1), multivalvar dysplasia (1); b) Cardiomyopathies ( 22 cases, $59 \%$ ): dilated (15) including 4 cases with isolated endocardial fibroelastosis and hypertrophic-restrictive (7); c) Myocarditis (5 cases, 14\%); d) Cardiac tumour (1 case, 3\%). There were 7 deaths (19\%): 4 early within 2 months and 3 late within 36 months. Cumulative survival was about $81 \%$ after 1 year, $77 \%$ at 3,5 and 10 years.

Early fatal outcome was higher in the congenital group with previous surgery and in pts undergoing transplantation in emergency conditions. One pt died because of graft viral myocarditis recurrence.

In conclusion, a wide spectrum of congenital and acquired cardiac disease are amenable to heart transplantation in pediatric age. The results are similar to those achieved in adults. Congenital heart disease with previous surgery and emergency transplantation are particularly at risk of operation failure. Since myocarditis may recur after transplantation, endomyocardial biopsy with molecular investigation is recommended either before or after operation.

\section{7}

Regional low flow perfusion during neonatal aortic arch surgery: relationship to repair

F.A. Pigula, S.K. Gandhi, R.D. Siewers, J. Tamblyn, S.A. Webber

E.M. Nemoto

Children's Hospital of Pittsburgh, Pittsburgh, USA

Introduction: We have previously shown that regional low flow perfusion (RLFP) can provide both cerebral and somatic circulatory 
upport to the neonate undergoing aortic arch reconstruction. We sresent our experience with RLFP as it relates to anatomic (2V) rersus palliative (1V) repair.

Methods: Thirty seven consecutive neonates (median age 6 days, ange 3-50) days) underwent arch augmentation between 998-20101 with RLFP, illustrated below. Twenty eight patients resented with hypoplastic left heart syndrome or variant $(1 \mathrm{~V})$, and 1) with biventricular anatomy (2V). Mean age at surgery was $9 \pm 8$ lays(range $3-5(1)$, mean weight was $3.1 \pm 0.5 \mathrm{~kg}$ (range 2-4.2). Jata are presented as mean(standard deviation).

Results: Refer to chart; $\mathrm{cpb}=$ cardiopulmonary bypass time, $: o x=$ aortic cross clamp time, cat $=$ circulatory arrest time, $2 \mathrm{LFP}=$ regional low flow perfusion time. ${ }^{\star} \mathrm{p}<0.05$, Student's test.

Jonclusion: RLFP is a flexible technique that can provide circuatory support to children requiring $1 \mathrm{~V}$ or $2 \mathrm{~V}$ repair. Children equiring $2 \mathrm{~V}$ repair require longer aortic cross clamp times, probibly as a consequence of intracardiac repairs. RLFP should reduce 1eurologically related morbidity and mortality in neonates requirng aortic arch surgery.

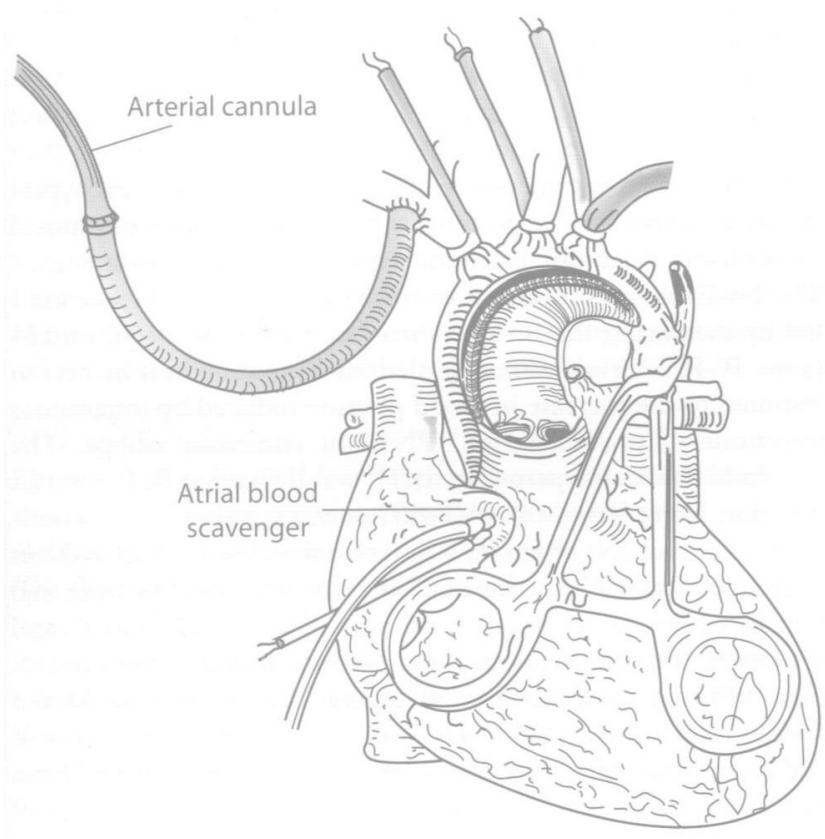

18

RV-PA-conduit versus modified BT-shunt in stage I palliation (Norwood Procedure or Modified Norwood Procedure)

R. Mair, E. Sames, G. Geiselseder, E. Lechner, G. Tulzer

General Hospital Linz, Dept of Surgery I, Linz, A

Background: Despite acceptable results in the Norwood prozedure shunt physiology remains a major problem in recovery after stage I palliation. Diastolic run-off nay lead to congestive heart failure, low diastolic blood pressure in the systemic circulation, impaired coronary, intestinal and cerebral perfusion. A conduit from the systemic ventricle to the pulmonary artery represents a banded physiology and avoids diastolic run-off and it's sequelae.

Methods: Since Nov. 1999, 30 patients underwent a stage I palliation for HLHS (24) or Single ventricle and systemic outflow tract obstruction(6). 21 patients got a RMBTS. In 9 patients a SVPA- conduit with a $5 \mathrm{~mm}$ PTFE prosthesis was constructed. Besides that the procedure was performed equally. Circulatory arrest was avoided or minimized by antegrade cerebral perfusion. There was no significant difference in age or weight between both groups.

Besides survival physiologic parameters as blood pressure, serum lactate, urine output were examined.

Results: Survival was $76 \%$ (16 of 21 pts.) in the BT-shunt group versus $88 \%$ in the SV-PA-conduit group ( 8 of 9 pts.). Diastolic blood pressure was significantly higher in the RV-PAconduit group compared to the shunt group (47 4,7 versus 36 $5,3 \mathrm{mmHg}$ ) $-\mathrm{p}=0,00093$ (unpaired t-test). Systolic bood pressure and urine output were also higher in the conduit group. Serum lactate concentration decreased faster in the conduit group to a level of less than $2 \mathrm{mmol} / 100 \mathrm{ml}(17,48,9 \mathrm{~h})$ compared to the shunt group $(26,411,7 \mathrm{~h})$.

Comments: The use of a SV-PA-conduit instead of a modified BT-shunt leads to a significant raise in diastolic blood pressure. Thus sytemic, cerebral and coronary perfusion are improved. Clinically the postoperative course was strikingly more stable in the conduit group. Basing on this experience we closed the chest primarily in the last 4 patients without any problems.

Avoidance of diastolic run-off after the Norwood procedure might result in lower postoperative morbidity and better survival.

\section{Session 3: Short Abstract Presentations 1}

19

Phase-contrast magnetic resonance imaging measurement of pulmonary venous blood flow in normal and surgically repaired pulmonary veins E.R. Valsangiacono, C. Barrea, C.K. Macgowan, J.S. Sinallhom, J.G. Coles, S.J. Yoo

University Children's Hospital Zurich, Cardiology, Zurich, $\mathrm{CH}$

Background: The pulmonary venous flow pattern (PVFP) is abnormal in presence of pulmonary vein (PV) stenosis or left ventricular filling abnormalities. Magnetic Resonance Imaging (MRI) not only is considered the new gold standard for anatomical evaluation of PV anomalies but can also provide information about blood flow pattern by using Phase Contrast (PC) cine MRI. Objectives: We sought to determine the PVFP by PC cine MRI in normal PV and surgically repaired PV, including unobstructed and stenotic veins, in a pediatric population.

Methods: We prospectively assessed the PVFP by PC cine MRI in 11 healthy children (age range 8-18 years, median 16 years) and 16 patients (age range 2 weeks-14 years, median 12 months) who underwent surgical repair for PV anomalies or presented native PV stenosis. The PVFP were analyzed qualitatively and quantitatively and compared among the following 3 categories: 1) normal; 2) surgically repaired without residual obstruction; and 3) obstructed PV.

Results: Flow velocities were measured by PC cine MRI in 23 normal PV, 51 repaired unobstructed PV and 11 stenotic PV. Normal and unobstructed PV showed biphasic PVFP with a peak in systole, a peak in the diastolic phase and an end-diastolic reversal curve. Repaired PV presented significant decreased peak systolic velocity $(p=0.001)$ and increased peak diastolic velocity $(p=0.005)$ with a decrease of the systolic/diastolic ratio $(p<$ 0.001 ). In presence of severe stenosis, PVFP were abnormal with blended systolic and diastolic peaks or with multiple minor peaks, representing turbulent flow.

Conclusion: PC cine MRI is capable of depicting PVFP changes under various anatomical and hemodynamic conditions, adding important functional information to the anatomical PV assessment provided by MRI. 
20

Cerebral function monitoring patterns during neonatal cardiopulmonary bypass

C.C. Geerts, N.E.M. Sins, A.N.J. Schouten, M.C. Toet, P.A. Hutter, N.M. Turner, A. Dodge-Khatami, G.B.W.E. Bennink, E.J. Meijboom Children's Heart Center, Wilhelmina Children's Hospital/LMC, Utrecht, $N L$

Introduction: Cerebral function monitoring (CFM) with singlechannel biparietal EEG electrodes is of prognostic value while assessing neurologic outcome. Sequences representing normal CFM patterns during neonatal cardiac surgery with cardiopulmonary bypass are as yet undefined. A normal CFM profile is proposed on the basis of satisfactory postoperative neurological outcome.

Methods: CFM was performed from 12 hours preoperatively, during, and up to 24 hours after routine cardiopulmonary bypass in 18 neonates undergoing cardiac repair (arterial switch; $\mathrm{n}=16$, truncus arteriosus, $n=1$, and arch hypoplasia; $n=1$ ). Specifically, different amplitude integrated EEG patterns by means of pattern recognition were looked at: continuous normal voltage $(\mathrm{CNV}=10-50 \mathrm{mV})$, tat tracing $(\mathrm{FT})$, and discontinuous normal voltage $(\mathrm{DNV}=$ predominantly above $5 \mathrm{mV}$ ), amongst others. Circulatory arrest was used in all but one patient.

Results: All patients survived with an uncomplicated postoperative course and satisfactory neurological outcome. Consistently, preoperative CFM showed a CNV pattern, circulatory arrest a FT pattern, and core rewarming a DNV pattern. Several hours after surgery, a CNV pattern with sleep-wake cycling returned. Excluding FT, the same sequence was found in the one patient who did not have circulatory arrest.

Conclusion: A standardised continuous perioperative CFM profile is proposed, defining a "normal" sequence during neonatal cardiac surgery. If validated, CFM anomalies could suggest ongoing intraoperative neurological insult, and potentially allow for appropriate modification of bypass strategy, or otherwise predict neurological morbidity early on. In patients with uneventful cardiac repair sustaining postoperative neurologic lesions, the "normal" intraoperative CFM sequence would exclude cardiopulmonary bypass as the causative factor of poor neurological outcome.

21

A heterozygous SCN5A point mutation in the domain

I-II linker leading to LQT syndrome with $2: 1$

AV block, ventricular hypertrophy, contraction and relaxation abnormalities

M. Geuillig, I. Witters, B. Eyskens, L. Mertens, T. Rossenbacker,

H. Heidbchel, X. Wehrens, R. Jongbloed, R. Kass

Kindercardiologie, Leuven, $B$

A foetus presented with 2:1 AV block, biventricular hypertrophy $(5.5 \mathrm{~mm}, 200 \%$ of normal), and a $5 \mathrm{~mm}$ ventricular septal defect. At birth major QTc prolongation up to $540 \mathrm{msec}$ was observed. The relationship between QT and R-R intervals indicated almost a doubling of the QT/R-R slope compared to an age-matched noncarrier, suggesting the possibility of an LQT-3 lesion. The ventricular wall was significantly hypertrophic with slow contraction ( $\max$ $290 \mathrm{msec}$ after QRS) and relaxation. Digoxin in combination with propranolol shortened the QTc interval, allowing 1:1 AV conduction; mexitil had no effect. Mutational analysis identified a missense mutation (L619F) in the domain I-II linker of the cardiac SCN5A $\mathrm{Na}+$ channel. The mother of the index patient also carried the L619F mutation but showed no QT lengthening. Whole-cell patch clamp analysis of recombinant L619F mutant channels expressed in HEK293 cells showed a persistent TTX-sensitive inward $\mathrm{Na}+$ current, reported as \% peak whole cell current, of $0.3 \%$ at $10 \mathrm{mV}$ vs. $0.1 \%$ in wild-type (WT: $0.2660 .06 \mathrm{pA} / \mathrm{pF}(\mathrm{n}=12) ; \mathrm{L} 619 \mathrm{~F}$
$0.790 .17 \mathrm{pA} / \mathrm{pF}(\mathrm{n}=9) ; \mathrm{p}<0.01)$. In addition, a $5.8 \mathrm{mV}$ positive shift in the steady-state voltage-dependence of channel availability caused an increase in the amplitude (WT: $0.580 .12 \mathrm{pA} / \mathrm{pF}(\mathrm{n}=7)$; L619F: $1.090 .17 \mathrm{pA} / \mathrm{pF}(\mathrm{n}=7) ; \mathrm{p}<0.05)$ and a shift in the peak voltage (WT: $-44.30 .7 \mathrm{mV}(\mathrm{n}=7)$; L619F: $-40.70 .9 \mathrm{mV}(\mathrm{n}=7)$ : $\mathrm{p}<0.01$ ) of $\mathrm{Na}+$ channel window-current.

Conclusions: This is the first genotyping of a patient with the complex cardiac phenotype of LQTS, AVB, hypertrophy and VSD. This study provides evidence that also a heterozygous mutation in SCN5A may cause LQT-3 with functional 2:1 AVB. The L619F mutation is the first LQTS mutation in SCN5A to occur in the cytoplasmic loop connecting domains I and II. The unique biophysical phenotype of the $\mathrm{L} 619 \mathrm{~F}$ mutation (combination of increased non-inactivating $\mathrm{Na}+$ current with a larger window current) may point to a novel role for the domain I-II linker in the $\mathrm{Na}+$ channel inactivation process. The mutation may lead to a complex LQT-3 variant. Unidentified genetic co-factors may modify its phenotypic expression.

22

Genetic transmission of vagal hyperreactivity in rabbits

A. Livolsi, J. Feldman, J. Fcingold, Y. Alembik, L. Itiss,

11. Fischbach, J. Messer, P. Bousquet

Service de Pédiarrie 1 -Chu de Hautepierre, Strasbunrg, F

Syncopal attacks in children are clained to be due to vagal hyperreactivity stimulated by parasympathetic stimuli.Our study aimed to put forward a possible genetic explanation for this pathology:

The bradycardic component of the baroreflex function was studied by measuring the $\mathrm{R}-\mathrm{R}$ intervals in ms on the electrocardiogram. R-R intervals were recorded sinchronously beat by beat in response to the increase in blood pressure induced by intravenous injections of phenylephrine (PNE) in conscious rabbits. The threshold of vagal hyperreactivity $(\mathrm{H})$ was defined as $\mathrm{R}-\mathrm{R}$ interval duration larger than $4000 \mathrm{~ms}$, whereas vagal activity was considered as normal (N) when $\mathrm{R}-\mathrm{R}$ interval was shorter than $4000 \mathrm{~ms}$ after injection of $500 \mathrm{~g} / \mathrm{kg}$ of PNE. We first selected male and female rabbits (Zika strain) with normal and exaggerated vagal responses, then hyperreactive and normal animals were crossed. The offsprings were obtained as follows: 1st group: male $\mathrm{H}$ and female $\mathrm{H}$ (10 pairs), 2nd group: male $\mathrm{N}$ and fenale $\mathrm{H}(6$ pairs). 3rd group: male $\mathrm{H}$ and female $\mathrm{N}$ (19 pairs), th group: male $\mathrm{N}$ and female $N$ (22 pairs). 261 male and 242 female offsprings of 12 weeks old were studied.

The rate of hyperreactive offsprings was higher in the $\mathrm{HxH}$ crossing (males $\mathrm{H}: 20 / 51(39.2 \%)$, females $\mathrm{H}: 20 / 48(41.6 \%)$ ) than in the $\mathrm{N} \times \mathrm{N}$ group (males $\mathrm{H}: 16 / 96$ (16.6\%). females $\mathrm{H}: 3 / 65$ (4.6\%)). Concerning males, the $\mathrm{H}$ rate was the higher when the amount of $\mathrm{H}$ parents was the higher too. In group 2 (males $\mathrm{H}$ : $8 / 36(22.2 \%)$, females $H: 1 / 39(2.5 \%)$ ) and 4 (males $H: 16 / 96$ (16.6\%), females $\mathrm{H}: 3 / 65(4.6 \%)$ ) low rate of $\mathrm{H}$ female offsprings was observed when male parents were $\mathrm{N}$.

In conclusion the functional analysis of the offsprings suggests that the inheritance of vagal hyperreactivity is oligogenic with an X-linked component.

23

Cardiac manifestations and complications in children with the acquired immunodeficiency syndrome at the Chris Hani Baragwanath Hospital, Johannesburg

F.F. Takaunira*, A.M. Cilliers

Chris Hani Baraguanath Hospital, Wibsey, Johannesburg, RSA

Introduction: Cardiovascular dysfunction is an important complication of the acquired immunodeficiency syndrome (AIIDS) that is 
being reported with increasing frequency. The exact prevalence of cardiac involvement in persons with AIDS is uncertain, particularly in the paediatric age group. The cardiovascular sequelae encountered with AIDS may be an expression of either primary cardiac infection or secondary systemic disease.

Materials and methods: A retrospective analysis of the spectrum of cardiac lesions associated with the human immunodeficiency virus (HIV) infection in children referred to the Division of Paediatric Cardiology at Chris Hani Baragwanath Hospital. The records of all HIV positive patients seen from January 1992 to June 1998 were reviewed.

Results: $96 \mathrm{HIV}$ infected patients were identified. Mean age $=$ 17.2 months. Male : Female ratio $=1.2: 1$. Vertical mode of transmission in all, but one patient. Reasons for referral were as follows: $34(35 \%)$ patients for cardiomegaly, 24(25\%) for a murmur, $20(21 \%)$ for CCF, $5(5 \%)$ for tachycardia and $3(3 \%)$ for suspected Infective Endocarditis. The reason for referral was not identified in $14(15 \%)$. The final diagnoses were: Cor pulmonale in 33(34\%), Pericardial Effusion $17(18 \%)$. LV Dysfunction in $11(11 \%)$, Congenital Heart Defects in 22(23\%), Sinus Tachycardia in $25(26 \%)$, Arrhythmias in 2(2\%) and Malignancy in $1(1 \%)$. Five $(5 \%)$ of the patients had documented cardiomegaly on chest X-Ray with no identifiable cardiac parhology. A normal cardiac evaluation was found in the remaining $16(17 \%)$ patients. Seventy one $(74 \%)$ of the patients had at least one documented episode of a chest infection.

Conclusion: There is a higher prevalence of Cor pulmonale in this series compared to other reports from the Western Literature.

Footnote: This research report was submitted to the Faculty of Health Sciences of the University of the Witwatersrand in part fulfilment of the MMed(Paediatrics) degree.

24

Quality of life in children with pulmonary atresia and intact ventricular septum (PA-IVS) in Sweden

B-.M. Ekmanjoelsson, L. Berntsson, J. Sunnegardh

The Queen Silvia Childrens Hospital, Cardiology, Skvde, S

Background: Pulmonary atresia and intact ventricular septum is a lethal congenital heart defect, the baby dying shortly after birth if left undiagnosed or untreated. Most patients undergo several surgical and/or catheter interventions neonatally and later in life. The patients are exposed to various physical and psychological challenges and also, their families are exposed to enormous stress and economic challenges. How does this affect quality of life compared to healthy children?

Methods: Quality of life was measured by a mailed questionnaire, taking into consideration the three life spheres of personal, interpersonal and external living conditions. Objective conditions and perceived subjective satisfaction are included in all dimensions. From January 1980 to December 1999, 84 children with PA-IVS were born in Sweden, the surviving $\mathbf{5} 2$ children received the questionnaire. This group of children, aged 2-20 years was compared to that of a random sample of 1851 Swedish children at the same ages without disabilities.

Results: +2 children and parents (80\%) answered the questionnaire. Compared to the reference group of healthy children the personal quality of life showed a significantly higher level of psychosomatic complaints $(p<0.001)$ and lower satisfaction with own activities and parent-child activities $(\mathrm{p}<0,(05)$. The activity level, self-esteem and peer acceptance were equal to the reference group. The interpersonal sphere showed a higher number of siblings $(p<0.05)$ and more available time in the patient group $(p<0.001)$, but the experience of relatives support was significantly lower $(p<0.05)$. The external sphere showed a higher income level $(\mathrm{p}<0.05)$ and higher satisfaction with work $(\mathrm{p}<0.05)$ than that of the reference group of healthy children, but there were no differences concerning education, profession, or housing conditions.

Conclusion: The children with PA-IVS were found to have more psychosomatic complaints and lower satisfaction with activity as compared to the reference group of healthy children, but the overall quality of life was equal between the groups.

25

Endocarditis prophylaxis in children with congenital heart disease: parent's awareness

J.L. Barreira, M.J. Baptista, J. Moreira, A. Azevedo, J. C. Areias

Department of Pediatrics, Hospital S. Joo, Porto, $P$

Background: Congenital heart disease (CHD) is the most common predisposing cause for childhood infective endocarditis (IE), accounting for $80 \%$ of cases. Knowledge about the disease and its complications is a key factor in promoting adherence to prophylaxis recommendations.

Purpose: To assess the level of understanding of the disease, IE risks and attitudes concerning oral health and antibiotic prophylaxis of IE among caregivers of children with CHD.

Patients and methods: Caregiver's knowledge was assessed during outpatient visit using a 15 -item questionnaire developed for this study. Answers were related with patient's age, cardiac condition and IE risk and respondent's age and level of education.

Results: Among 97 approached caregivers, 83 completed the questionnaire. The mean age of patients was $7,75,9$ years (range 1-24 years). Eleven (13\%) patients were at high risk for IE and $30(36 \%)$ at moderate risk. Most respondents (80\%) alleged to know patients cardiac condition but only $37 \%$ described it correctly. Twenty-nine respondents $(35 \%)$ were aware of IE risk, while only 13 related that risk to dental procedures. Knowledge of IE risk was associated with respondent's level of education ( $p=0,03$ ) but not with respondent age or patient actual risk of IE. Most respondents (76\%) recalled having been advised on oral hygiene measures and declared to brush teeth at least once a day $(92 \%)$. Forty-three children $(52 \%)$ had ever been to the dentist and 27 did so in the previous six-month period. Almost all (42 of 43) informed the dentist about child's CHD, but only $20(48 \%)$ reported having received antibiotics before dental procedures. Antibiotic prophylaxis was associated with patient's age $(p=0,04)$ but not with level of education of the respondent or actual risk of IE. Being aware of IE risk was significantly associated with better oral hygiene $(p=0,001)$ and more frequent dental appointments $(p=0,03)$, independently of the level of education.

Discussion: The results of this survey support the need of reinforcement about IE risk information and prophylaxis recommendations among caregivers of children with CHD.

\section{6}

Serious complications after implantation of SynerGraft valved conduits in the pulmonary position

U. Salzer-Muhar, E. Kitzmller, M. Marx, M. Schlemmer, P. Simon, G. Weigel, G. Amann

Division of Pediatric Cardiology; Department of Pediatrics, Vienna, $A$

We want to report our preliminary experience (04/200112/2001) with SynerGraft valved conduits (CryoLife Inc.) and to draw attention to potential fatal complications. SynerGrafts are constructed from porcine valve leaflets that have been treated to remove all histologically demonstrable leaflet cells and to reduce porcine cell related immunoreactivity.

Patient 1 - a 9 year old boy (PA/VSD) - had developed severe stenosis of an aortic homograft in the pulmonary position within 
16 months. The implantation of a $15 \mathrm{~mm}$ aortic SynerGraft (model 500) was seen as a suitable alternative. Within five weeks the gradient at the distal anastomosis increased from 18 to $77 \mathrm{mmHg}$. Reoperation was scheduled, but had to be postponed because of viral infection. The boy died suddenly of right ventricular failure. Histologic examination of the graft revealed a severe inflammatory response

Patient 2 - a 3 year old boy (critical AS) in whom a Ross-Konno procedure with implantation of an $14 \mathrm{~mm}$ aortic SynerGraft (model 500) had been performed - developed a moderate narrowing of the graft, 7 months postop the gradient is stable at $44 \mathrm{mmHg}$ Patient 3-a 7 year old boy (common arterial trunc) - was reoperated because of homograft stenosis (Doppler gradient $92 \mathrm{mmHg}$ ) and a $17 \mathrm{~mm}$ pulmonary Syner Graft (model 700) was implanted. Postoperative echocardiography confirmed normal flow across the graft. On the 8 th postoperative day while still monitored on the ward, the boy died suddenly during sleep, resuscitation was unsuccessful. Autopsy revealed a rupture in the anterior aspect of the SynerGraft. The rupture was located in the tubular part at the height of the commissures. Histologic examination showed a severe inflammatory response, detailed results are pending.

Patient 4 - a 4 year old boy (critical aortic stenosis) had undergone a Ross procedure with implantation of a $16 \mathrm{~mm}$ pulmonary SynerGraft (model 700) the day before patient 3 expired. After a frank discussion with the parents prophylactic explantation of the graft was performed and an oversized $22 \mathrm{~mm}$ pulmonary homograft was implanted.

The concept of bioengineered xenografts is an attractive one. From our preliminary experience however we would not recommend the use of those prostheses in the young population until there are convincing data concerning graft immunogenicity

\section{Session 4: Fetal}

27

Kartagener syndrome is an heterogeneous condition that may be associated to axonemal dynein intermediate chain gene (DNAI1) mutations

C. Guichard, M-C. Harricane, J-J. Lafitte, J. Godard, M. Zaegel,

V. Tack, G. Lalau, P. Bouvagnet

Universite Claude Bernard Lyon 1, Lyon, F

Kartagener syndrome is a trilogy of symptoms (nasal polyps, bronchiectasis and situs inversus totalis) that is associated with ultrastructural anomalies of cilia of epithelial cells covering the respiratory tracts and eventually flagella of spermatozoa.

We screened for mutation the axonemal dynein intermediate chain gene 1 (DNAI1) in a series of 34 Kartagener patients. This gene which was previously demonstrated to be responsible for a case of Primary Ciliary Dyskinesia (PCD) without situs inversus is composed of 20 exons and encodes a 699 amino acid protein It is localized on 9p13-p21. The gene was screened for mutation by PCR, SSCP and eventually sequencing.

Three cases were found to have mutations in the DNAI1 gene. Each individual was heterozygous for a common loss-of-function: an A insertion at position 3 of intron 1 donor site that results in absence of splicing and peptide truncation. In addition to this mutation, patient 1 had a transition at position $1543(G>A)$ that resulted in changing a highly conserved glycine to a serine. Moreover, patient 1 has a brother who suffers also from recurrent upper and lower respiratory tracts infections and sterility but no situs inversus. Repeated spermograms demonstrated immotile flagellum of spermatozoa. The cilia showed absent or truncated outer dynein arm. The PCD brother had the same compound mutations as the proband.
Patient 2 and her sister who suffers from PCD with no situs inversus have both the same compound mutations as patient 1 . Both of them had children.

Patient 3 who has no affected sibling has a 12 bp deletion that results in shortening DNAI in the 4th WD domain. Patient 3 has in addition to situs inversus totalis, chronic sinusitis, bronchitis, recurrent otitis and aplasia of frontal sinus.

In conclusion, we denionstrate that mutation in DNAI1 may result in Kartagener syndrome and hence laterality disturbance. About $9 \%$ of Kartagener cases are secondary to DNAI1 mutation. By contrast, the other $91 \%$ had no mutations strongly suggesting that Kartagener is as PCD a heterogeneous condition. The A insertion in intron 1 is highly prevalent since it represents $50 \%$ of DNAI1 mutations so far reported in PCD and Kartagener patients.

\section{8}

Genome wide gene expression profile analysis of congenital malformed hearts

S. Sperling, B. Kaynak, A.v. Heydebreck, D. Seelow, R. Pregla, S. Mebus, H. Lehrach

Max-Plnack-Institut for Molecular Genctics, Berlin, D

Congenital heart defects (CHD) are the manifestation of anomalies in embryonic cardiac development. According to the morphologic and hemodynamic features of the different $\mathrm{CHDs}$, a cardiac remodeling process for adaptation to volume and/or pressure overload etc. is taking place. Recent advances of microarray technology enabled us to identify characteristic gene expression profiles for a variety of hemodynamic conditions in human using genome wide microarrays.

Methods: Total RNA from atrial or ventricular myocardial probes $(\mathrm{n}=50)$ of patients with atrial septal defect Type II (ASDII), double outlet right ventricle (DORV), tetralogy of Fallot (TOF), ventricle septal defect with pulmonary atresia (VSD-PAS) and controls (C) have been radioactive labeled and hybridised to Human Unigene II array membranes (Resource Center Berlin, PCR products of 75.000 different IMAGE clones). Images have been analysed using in-house $\mathrm{X}$-digitize software. Normalisation and statistical analysis were carried out using matlab tools with scripts generated in-house. Differential gene expression was assumed with a p-value $<0.001$

Results: Using high density arrays with 75.000 expression informations we could identify $\mathbf{5 8 0 5}$ genes expressed at middle till high levels in all studied probes (diseases and controls), of which 371 genes have been differentially expressed. Additional 2200 genes with low expression levels and characteristic profiles have been identified and will be presented in detail. These genes are differentialy expressed between different CHDs compared with controls (table 1a) and/or genes preferentially expressed in normal myocardium of one of the four heart chambers as well as age and sex specific genes (table $l b$ ).

To our knowledge this is the first study using a genome wide array approach to identify gene expression profiles characterising CHDs with volume and/or pressure overload as well as the normal myocardium of the four heart chambers. The data we will present should contribute to our understanding of cardiac adaptation processes and function.

Table 1a: Example of gene counts showing characteristic profiles.

Table 1b: R - right, L - left, A - atrium, V - ventricle. 
29

Familial recurrence of congenital heart disease in a cohort of 6647 consecutive pregnancies assessed by fetal echocardiography

H. Gill, M. Splitt, G. Sharland, J. Simpson

Kings College London, London, UK

Introduction: There is little data on the recurrence of congenital heart disease (CHD) based on fetal echocardiographic series. Fetal series will include pregnancies which end in intra-uterine death or termination, unlike postnatal series, leading to more complete ascertainment of cases.

Methods: Retrospective analysis of referrals to a tertiary fetal cardiology centre in the UK, between January 1990 and December 1999. Data was obtained from a prospectively acquired computerised database. We reviewed all cases where referral indication included CHD in a first degree relative.

Results: 6647 pregnancies were assessed in view of a first degree family history of CHD. The history was maternal, paternal or sibling in 1121,374 and 5152 cases respectively. 169 cases of recurrence were identified $(2.5 \%)$. For maternal, paternal or sibling $\mathrm{CHD}$ the recurrence risks were $2.2 \%, 2.7 \%$ and $2.4 \%$ respectively. Where the index case was of left heart obstruction $(n=39)$, the recurrence lesions were hypoplastic left heart $(\mathrm{HLH})(\mathrm{n}=9)$, coarctation of the aorta (CoA) $(n=10)$, aortic stenosis $(n=2)$, VSD $(\mathbf{n}=12)$, transposition of the great arteries (TGA) $(n=1)$ and tricuspid atresia $(n=2)$ and others $(n=3)$. Where the index lesion was TGA $(n=6)$ the recurring lesions were TGA $(n=2)$, $\operatorname{HLH}(\mathrm{n}=1), \operatorname{CoA}(\mathrm{n}=1)$ and VSD $(\mathrm{n}=2)$. For AVSD $(\mathrm{n}=4)$ the recurring lesions were AVSD $(\mathrm{n}=3)$ and tetralogy of Fallo $(\mathrm{n}=1)$.

Conclusions: To our knowledge, this is the largest reported prenatal series examining familial recurrence of $\mathrm{CHD}$. It confirms that pregnancies with a first degree history of $\mathrm{CHD}$ are at increased risk of recurrence, regardless of whether the index case is maternal, paternal or sibling. There was significant under-referral of cases with a paternal history of $\mathrm{CHD}$. The recurrence lesions are frequently, but not exclusively of the same type as the index case. Our data does not accord with a recent UK study suggesting a higher recurrence following maternal $\mathrm{CHD}$, nor that TGA is a sporadic condition (Burn et al 1998).

\section{0}

Doppler tissue imaging enables assessment of fetal right ventricular function

H. Gardiner, J. Wolfenden, W. Lei, M. Henein

Royal Brompton Hospital, London, UK

Background: Assessment of right ventricular (RV) function is made more difficult because of its complex morphology. RV free wall amplitude and velocities have been shown to correlate with its overall function (in adults) particularly in systole, as assessed by ejection fraction. Transtricuspid flow velocities, usually taken as a marker of RV diastolic function, are not helpful in the fetus as the early to late filling velocity ratio is normally reversed.

Aim: To examine the feasibility of assessing fetal RV free wall myocardial function at different gestational ages using its long axis amplitude of motion and to compare it with transtricuspid flow velocities.

Methods: We examined 43 fetuses; 13 (20-23 weeks), 4 (24-27 weeks), 7 (28-31 weeks), 11 (32-35 weeks) and 8 (36-39 weeks) using long axis amplitude ( $\mathrm{M}$-mode) and shortening \& lengthening velocities (DTI).
Results: RV systolic velocities correlated with the progressive increase in the free wall amplitude of motion with increasing gestation, $\mathrm{R}=0.60, \mathrm{p}<0.01$. This was associated with a progressive gestational increase in $R V$ outflow tract velocities, $p<0.03$. Early $(p<0.001)$ and late $(p<0.01)$ diastolic free wall velocities increased with age along with the corresponding increase in transtricuspid flow velocities $(\mathrm{p}<0.001 \& \mathrm{p}<0.04)$.

Conclusion: It is feasible to evaluate RV systolic and diastolic function during intrauterine life, particularly in the second half of pregnancy. RV amplitude and velocities increase with fetal maturity up to 32-36 weeks, but appear stable thereafter. These findings form the basis for normal fetal right ventricular data that can be applied in disease states.

Right Ventricular Function

\begin{tabular}{|c|c|c|c|c|c|c|c|c|c|}
\hline \multirow[b]{3}{*}{ Variable } & \multicolumn{9}{|c|}{ Gestation } \\
\hline & \multicolumn{2}{|c|}{$20-23.9$} & \multicolumn{2}{|c|}{$24-27.9$} & \multicolumn{2}{|c|}{$28-31.9$} & \multicolumn{2}{|c|}{$32-35.9$} & $36-39.6$ \\
\hline & Mean & SD & Mean & SD & Mean & SD & Mean & $\mathrm{SD}$ & Mean SI \\
\hline $\mathrm{m}$ & 5 & 1.2 & 7.5 & 0.5 & 6 & 1.6 & 7.2 & 1.1 & 7.2 \\
\hline Am & 10.3 & 2.0 & 11.2 & 2.6 & 10.2 & 2.9 & 11.9 & 1.9 & $11.9 \quad 1$. \\
\hline $\mathrm{Em} / \mathrm{Am}$ & 0.55 & & 0.67 & & 0.59 & & 0.61 & & 0.61 \\
\hline $\mathrm{Sm}$ & 7.2 & 2.8 & 6.4 & 0.9 & 6.9 & 2.8 & 8.2 & 1.8 & 8.2 \\
\hline & 30.2 & 5.0 & 37.6 & 4.5 & 35.6 & 7.1 & 42.4 & 4.3 & 42.4 \\
\hline 9 & 50 & 7.1 & 60.6 & 8.7 & 51.5 & 11.5 & 58.2 & 7.0 & 58.2 \\
\hline$E / A$ & 0.60 & & 0.62 & & 0.69 & & 0.73 & & 0.73 \\
\hline LAX & 5.1 & 1.0 & 6.1 & 0.9 & 7.2 & 1.1 & 8.0 & 1.7 & 8.0 \\
\hline
\end{tabular}

Em: Early diastolic DTI (cm/s), Am: Atrial diastolic DTI $(\mathrm{cm} / \mathrm{s})$ Sm: Systolic DTI $(\mathrm{cm} / \mathrm{s})$, E: Early ventricular filling $(\mathrm{cm} / \mathrm{s})$, A: Atrial ventricular filling $(\mathrm{cm} / \mathrm{s})$, Lax: Long axis function $(\mathrm{mm})$, DTI: Doppler tissue imaging.

\section{1}

Real-time $3 \mathrm{D}$ versus $2 \mathrm{D}$ echocardiography in fetuses with normal and abnormal hearts

G. Tulzer, W. Arzt*

Children's Hospital of Linz, Dept of Pediatr. Cardiol, Linz, A

Real-time 3D echocardiography is thought to be a better method to investigate a complex three-dimensional structure like the human heart. To test clinical feasibility and usefulness of real-time $3 \mathrm{D}$ echo in human fetuses we compared this method to conventional 2D echo in 20 consecutive, unselected fetuses with normal and 10 fetuses with abnormal hearts. Diagnoses encompassed at least one of the following: transposition of the great arteries: $(n=4)$; hypoplastic left heart syndrome $(n=3)$; double outlet right ventricle $(n=2)$; tricuspid atresia $(n=1)$; critical aortic stenosis: $(\mathbf{n}=2)$, tetralogy of Fallot $(n=1)$. After acquisition of a real-time 3D data-set (duration 1 to 5 seconds; 16 frames/second; 4 to 45 megabyte) it was stored on a remote computer for later off-line analysis. Acquisition-time was between 0,5 to 2 minutes. Off-line analysis (done by a different person, not aware of the $2 \mathrm{D}$ examination result) included identification of: venous connections, 4-chamber view, atrio-ventricular and ventricular arterial connections, aortic and ductal arches. In normal fetuses off-line analysis lasted on average 3 to 4 minutes, in fetuses with congenital heart disease 15 to 40 minutes. Assessment of the 4 -chamber view and outflow tracts was excellent and comparable to the $2 \mathrm{D}$ echo in $20 / 20$ normal fetuses, arches in $12 / 20$, venous connections in $10 / 20$. In fetuses with abnormal hearts, the main pathology was clearly detectable in all. Compared to $2 \mathrm{D}$ echo assessment of chamber size, AV valves, papillary muscles and VSD relationship was superior or equal, great vessel anatomy could not be assessed in 1 and was otherwise inferior or equal. New views provided 
additional information in $2 / 10$ fetuses. Real-time 3D echo was reliable for 4-chamber view and outtlowtract screening. Advantages over $2 \mathrm{D}$ examination are that it may significantly shorten examination time, improves patient comfort, provides excellent data storage and enables electronic data transfer to remote experts, who can examine the fetal heart in real-time. In abnormal hearts it may add additional information due to new views and $3 \mathrm{D}$ reconstructions, which can be rotated in space. Limitations were slow frame rate, low lateral resolution and lack of (color) Doppler.

32

Cardiac malformations in fetuses with increased nuchal translucency: ultrasound diagnosis and post-mortem morphology

M.C. Haak, M.M. Bartelings, J.M.G. van Vugt,

A.C. Gittenberger-de Groot

Department of Anatomy and Embryology, Leiden University $M C$, Leiden, $N L$

Introduction: Nuchal translucency (NT) measurement in first trimester pregnancy has become an established method for identifying fetuses at risk for aneuploidy. The high occurrence of heart malformations in fetuses with an enlarged NT, isolated or as result of aneuploidy has led to the theory that impaired cardiac function due to heart malformations might play a role in NT enlargement. In this study the diagnostic accuracy of transvaginal echocardiography (TVEC) in fetuses with a NT $>$ p95 is explored, by comparing the ultrasound diagnosis with the postmortem examination (PME) or the follow-up. The second aim was to determine the type of heart malformations in these fetuses.

Methods: In 45 first trimester pregnancies with a NT > p95, TVEC was performed. Ultrasound diagnosis was compared to PME. Mean NT in the fetuses with and without heart defects was calculated.

Results: In 10 of the 45 fetuses (22\%), heart malformations were suspected by ultrasound. Of these ten, eight had a chromosomal abnormality $(80 \%)$. PME showed minor additional findings in some cases, but in none major discrepancy's occurred. (A)VSD's were the most common heart defects in trisomic feruses. In three fetuses with $45 \mathrm{X}$ a HLHS was diagnosed. Heart defects were diagnosed in all three euploid fetuses in which fetal demise occurred. The sensitivity and specificity of TVEC compared to PME in this study was $88 \%$ and $97 \%$. The mean NT in fetuses with a normal heart was significantly smaller, compared to fetuses with a heart defect $(4.3$ and $7.4 \mathrm{~mm}, \mathrm{p}<0.001)$

Conclusion: TVEC can be performed reliably in fetuses with an increased NT. Heart malformations are diagnosed correctly, which can reduce uncertainty in parents. If a heart defect is diagnosed in these fetuses, the risk on an unfavorable outcome is increased, as fetal demise occurred in all euploid fetuses with a heart malformation. These data do not support the theory of cardiac failure leading to NT enlargement, due to the type of heart defects found. These are unlikely to cause hemodynamic changes. Abnormal endothelial development might be the common pathophysiological pathway leading to cardiac defects, lymphatic jugular maldevelopment and subsequently NT enlargement.

33

The relationship between increased fetal nuchal translucency in the first trimester and second trimester cardiac echogenic foci

F. Prefumo, F. Presti, B. Thilaganathan, J.S. Carvalho

Royal Brompton Hospital, London, UK

Background and objective: Increased nuchal translucency (NT) thickness is strongly associated with chromosomal abnormalities.
Echogenic foci (EF) are possible markers for these anomalies. We have tested the hypothesis that increased first trimester NT is associated with isolated intracardiac EF foci in the second trimester.

Design and methodology: Observational study in a fetal medicine unit. All pregnancies from our local catchment area booked and delivered between January 1997-June 2000 were identified from a computerised database. Institution-based reference range (centiles) from a larger group screened at our centre was used. 7686 singleton fetuses had NT scan and either a subsequent normal anomaly scan at $18-23$ weeks $(\mathrm{n}=7447)$, or an isolated intracardiac $\mathrm{EF}$ $(n=239)$. Aneuploid fetuses and those with structural abnormalities or multiple soft markers were excluded. NT measurements in the two groups (with and without EF) were compared with the reference range and odds ratio for presence of $E F$ in the second trimester based on first trimester NT were calculated.

Results: Among 259 fetuses with NT measurements $>95$ th percentile, 21 (8.1\%; $95 \% \mathrm{CI}, 5.1$ to $12.1 \%)$ subsequently demonstrated cardiac EF but only 218 of 7727 fetuses with normal NT measurements $(2.9 \% ; 95 \% \mathrm{CI}, 2.6$ to $3.3 \%)$ showed this finding on later scans. The odds ratio for displaying EF in the second trimester in fetuses with an increased NT was 2.92 when compared to fetuses with a normal NT measurement $(95 \% \mathrm{CI}, 1.83$ to 4.65$)$. Conclusions: There is a significant association between first trimester raised NT measurements and increased prevalence of isolated intracardiac EF in the second trimester in a large unselected population of otherwise normal fetuses. Previously. increased NT measurements have been reported to be associated only with conditions implying a severe or guarded prognosis such as chromosomal abnormalities, structural defects or a series of genetic syndromes. This is the first description of the association between increased NT measurements and an apparently benign condition without known short or long term problems. This suggests a common pathogenetic mechanism for the occurrence of both increased NT and EF which may be related to transient altered fetal haemodynamics in the first trimester of pregnancy.

\section{4}

Pulmonary venous flow in fetuses with hypoplastic left heart syndrome and restrictive foramen ovale C. Barrea, M. Taketazu, J.F. Smallhom, L. K. Hornberger

Cliniques Universitaires Saint Luc-UCL, Brussels, $B$

Restrictive foramen ovale (FO) with severe left atrial hypertension (LAH) places a newborn with severe left heart obstruction (LHO) at high risk of mortality, but in our experience, direct evaluation of the FO size may be difficult at fetal echocardiography. We hypothesized that prenatal pulmonary venous (PV) flow patterns in $\mathrm{LHO}$ reflect severity of LAH. We reviewed fetal and neonatal echocardiograms and clinical records of all fetuses diagnosed with LHO between May 1999 and November 2001. FO size, PV and pulmonary artery (PA) diameters, and PV flow patterns were assessed. Forty-four fetuses with LHO were identified (initial age 256 weeks), PV spectra were available in 39 . There were 22 pregnancies terminated and 22 live births of whom 21 had follow-up. At initial exam, 3 PV flow patterns were observed (Fig.): (a) continuous forward flow with low velocity a wave reversal $(<0.5$ of forward velocity) $(\mathrm{n}=24)$, (b) continuous forward flow with high velocity a wave reversal $(n=10)$, (c) brief to and fro flow $(n=5)$. All fetuses with (c) flow pattern had significant PV dilatation with $\mathrm{PV} / \mathrm{PA}$ diameter ratio of 1.430 .49 versus (a) 0.750 .19 and (b) 0.720 .17 . Of the 39,20 continued to term: 11 with (a), 6 with (b) and 3 with (c) patterns. All fetuses in group (c) had an intact atrial septum (including 1 whose FO could not be evaluated prenatally). 
were profoundly cyanotic at birth and underwent creation of an atrial septal defect within 1-2 hours of life. Two patients in (a) and (b) had also an intact atrial septum but had significant decompressing collateral veins and did not require urgent atrial septectomy. There was no correlation between the fetal FO size and the neonatal FO size or the neonatal clinical condition but well between the PV flow pattern and the outcome.

Conclusions: Prenatal PV flow patterns in LHO provide insights into the pathogenesis of PV changes found in infant with restrictive FO, and identify the fetuses at risk for severe LAH at birth. This information is critical for prenatal counseling and planning of perinatal management.

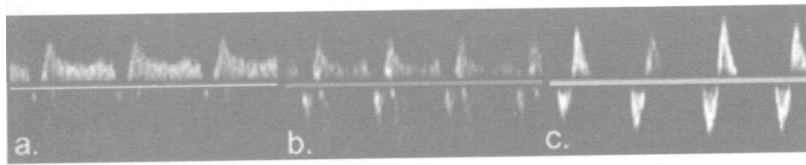

35

Experiences with the AEPC research protocol in fetuses with bradyarrhythmias and complete heart block R. Oberhoffer, M. Tollens, D. Lang, K.T.M. Schneider Pediatric Cardiology, Childrens Hospital Techn. Univ, Munich, D

Complete heart block is claimed to have a neonatal incidence of $1: 22000$. Even if prenatal diagnosis is accurately established by means of fetal echocardiography, outcome with or without therapy remains unclear. We report on our experience in 22 pregnancies referred between 1993 and 2001 for exclusion or diagnosis of fetal bradyarrythmias.

Patients and methods: The median gestational age at the time of referral was 25 weeks (range 19-38w). The first and the follow-up examinations were performed according to the sequential segmental analysis and by means of $\mathrm{M}$-mode-echocardiography for simultaneous registration of atrial and ventricular rates. They were documented on videotape together with signs of cardiac compromise (cardiomegaly, effusions). Maternal anti-Ro-antibodies were measured using ELISA. The AEPC reseach protocol was offered to all women with collagenosis.

Results: Of the 22 fetuses, 14 showed bradyarrhythmias: 3 blocked PACs, 2 intermittend variable blocks (both forms partly proceeding), 9 complete heart block. The remaining 8 fetuses of mothers with collagenosis had normal sinus rhythm; in 5 cases, Ro-antibodies were found, in 3 of them, mothers voted for prophylactic corticoid treatment. All babies survived without any problems. Of the 14 bradyarrythmic fetuses, 7 showed severe concommitant structural heart defects, and 7 a structurally normal heart in the setting of maternal Ro-antibodies. Only one infant of those with abnormal hearts survived, but 5 of those with maternal collagenosis (2 pacemakers). Prenatal treatment with corticosteroids in 6 of these fetuses did neither influence the degree of heart block nor the ventricular rate. Randomization according to the AEPC-protocol was not possible, as mothers voted for immediate medication.

Conclusion: In this study, bradyarrhythmias were equally distributed in fetuses with congenital heart defect and with maternal collagenosis. Outcome was better in the latter group; corticoid treatment did not seem to change the rhythm disturbances. Exact assessment of its efficiacy, however, was not possible, because acceptance of the AEPC protocol for fetuses with complete heart block was poor.

\section{Session 5: General}

36

The value of $\mathrm{QT}$ dispersion and the rate-corrected $Q T$ interval in detecting children with mitral valve prolapse susceptible to ventricular tachycardia: a prospective study

W. Bobkowski, A. Siwinska, J. Zachwieja, B. Mrozinski,J. Maciejeuski Department of Pediatric Cardiology, Poznan, PL

Mitral value prolapse (MVP) is one of the most common form of valvular cardiac disease in children and adolescence. Several studies indicate a high prevalence of ventricular arrhythmias (VA) in these patients. This study had two aims: (1) to compare the ratecorrected QT (QTc) interval and QT dispersion (QTd) in children with MVP with those in age and sex-matched controls, and (2) to examine prospectively the relation between QTc and QTd and ventricular tachycardia (VT) in children with MVP. 151 children with MVP (aged: 12.23.1 years) and 165 healthy subjects (aged: 12.33 .7 years) were examined. All children underwent standard ECG $(50 \mathrm{~mm} / \mathrm{s}$ speed), 24-h ambulatory ECG monitoring and echocardiography. The children with MVP were followed prospectively for a mean of 64 months. Follow-up studies involved clinical examinations, standard ECG and 24-hour ambulatory ECG monitoring at maximum intervals of 6 months. The children with MVP had a significantly higher prevalence of VA than the controls $(42 \%$ vs. $13 \%, \mathrm{p}<0.0001)$. QTd $(3418 \mathrm{~ms}$ vs $227 \mathrm{~ms}, \mathrm{p}<0.00001)$ and QTc interval $(40421 \mathrm{~ms}$ vs. $39318 \mathrm{~ms}$, $\mathrm{p}<0.00001)$ were greater in children with MVP than in controls. A marked increase in QTd was recorded in children with MVP and VA compared with those without VA (4419 ms vs. $1714 \mathrm{~ms}, \mathrm{p}<0.00001)$. During follow-up, 24 children with MVP developed nonsustained VT (3.1/100 subject-years). To identify the children with MVP who developed nonsustained VT the algorithm that combines QTd $\geqslant 50 \mathrm{~ms}$ and QTc interval $\geqslant$ $410 \mathrm{~ms}$ had a sensitivity of $81.3 \%$ and a specificity of $92.4 \%$ (positive predictive value: $68.4 \%$; negative predictive value: $96.1 \%$ ). It was concluded that a simple algorithm that combines QTd with the QTc interval may prove useful in determining which children with MVP are at risk of developing nonsustained VT. A high specificity and high negative predictive value indicate that those children with MVP with a QT dispersion $<50 \mathrm{~ms}$ and a QTc interval $<410 \mathrm{~ms}$ are at low risk of developing VT

37

Impaired response to dobutamine and amrinone of the adult isolated perfused heart following neonatal hypoxia in the rat

C.V. Rohlicek, S. Viau

Montréal Children's Hospital/McGill University, Montréal, CDN

We have asked whether a transient period of hypoxia neonatally $\left(\mathrm{FiO}_{2}=0.12\right.$, days $1-10$ of life $)$ in the rat has long-lasting effects on the cardiac response to inotropic stimulation with the adrenergic agonist dobutamine or the phosphodiesterase inhibitor amrinone in later life. Experiments were conducted on the isolated perfused hearts of 48 adult Sprague-Dawley rats $(4367 \mathrm{~g}, 902 \mathrm{~d}$ (SEM)). Twenty-four animals had experienced hypoxia neonatally (Neonatally Hypoxic) while 24 others had not (Control). Excised hearts from these animals were perfused in the Langendorff mode at a constant pressure of $100 \mathrm{~cm} \mathrm{H}_{2} \mathrm{O}$ with modified KrebsRinger solution warmed to $37^{\circ} \mathrm{C}$. A water filled balloon connected to a pressure transducer was placed in the left ventricle for measurement of left ventricular pressure (LVP), and the maximum 
rate of pressure increase $(+\mathrm{dP} / \mathrm{d}$ tmax $)$. In 14 animals from each group dobutamine was added to the perfusate at an initial concentration of $0.1 \mathrm{M}$ and the concentration increased sequentially to $0.2 \mathrm{M}, 1 \mathrm{M}$, and $10 \mathrm{M}$ every 5 minutes. In 10 further animals from each group amrinone was added to the perfusate at a concentration of $47 \mathrm{M}$. The positive response of peak LVP and $+\mathrm{dP} / \mathrm{dtmax}$ to stimulation with dobutamine and amrinone was substantially impaired in the Neonatally Hypoxic rats compared to the Control animals. Significant differences were found between the two groups at dobutamine concentrations of $1 \mathrm{M}$, and $10 \mathrm{M}$ (LVP: Control 1987\%, 2169\% Neonatally Hypoxic 1728\%, 1878\%; +dP/dtmax: Control 2016\%, 2249\%; Neonatally Hypoxic $1778 \%, 1979 \% \mathrm{P}<0.05)$ as well as during stimulation with $47 \mathrm{M}$ amrinone (LVP: Control 1251\%, Neonatal Hypoxia $1152 \%$; +dP/dtmax: Control 1303\%; Neonatally Hypoxic $1176 \% \mathrm{P}<0.05)$. These results indicate a long-lasting impairment of the cardiac contractile response to inotropic stimulation with dobutamine and amrinone following a transient period of hypoxia neonatally. Our finding of a decreased response to both an adrenergic agonist as well as a phosphodiesterase inhibitor suggests the possibility of persistent alterations in the intrinsic ability of the myocardium to respond to inotropic stimulation. Our results may have important implications for the post-operative management of patients born with cyanotic heart defects.

\section{8}

Pulmonary artery remodeling in transposition of the great arteries: relevance for neo-aortic root dilatation S. Lalezari, M. G. Hazekamp, M.M. Bartelings, P.H. Schoof,

A. C. Gittenberger-de Groot

Liden University Medical Center, Leiden, NL

Background: Transposition of the great arteries (TGA) is currently treated with the arterial switch operation. Dilatation of the neo-aortic root is a late complication. Vascular remodeling might play an important role in this process. So far, no reports have been made about structural differences in the vessel wall and sinus between TGA and normal hearts. Therefore, samples of the aorta and pulmonary artery of patients with untreated TGA were investigated and compared to normally related great arteries.

Material and methods: Aortic and pulmonary artery vessel wall and sinus samples were taken from 20 unoperated human TGAheart specimens and 9 age matched, normal post-mortem human heart specimens. The specimens were divided in two groups according to age. Routine histology was performed as well as immunohistochemical staining for smooth muscle cell differentiation markers $1 \mathrm{~A} 4, \mathrm{a}-\mathrm{SM} 22$ and calponin. The samples were observed using light microscopy.

Results: Routine histology revealed structural differences between early normal aorta and pulmonary artery which became more pronounced in the late group. In the early group in TGA, no striking differences were seen between the aorta and pulmonary artery. With increasing age, a marked downregulation of all smooth muscle cell markers was observed in the pulmonary artery, as opposed to the aorta.

Conclusions: In the pulmonary artery of untreated TGA, there is a dedifferentiation of smooth muscle cells with increasing age. This difference in vessel wall morphology between normal and transposed aorta and pulmonary artery suggests that the vessels in TGA are not only transposed but structurally different. These results provide an explanation for the neo-aortic root dilatation that has been reported as a late complication of the arterial switch operation.
39

Inotropic effects of selective ET-B receptor stimulation and its modulation by the endocardial endothelium

A. Leite-Moreira, C. Brs-Silva, C. Pedrosa

Dept. of Physiology, Faculty of Medicine, Porto, $P$

Background: Endothelin-1 (ET-1) is an endogenous peptide whose effects are mediated by ET-A and ET-B receptors. ET-A are responsible for the vasoconstrictor. positive inotropic and growth promoting properties of ET-1, while ET-B for its vasodilator and anti-mitogenic effects, presumably due to NO release. The effects of selective ET-B receptor stimulation on myocardial contractility were investigated.

Material and Methods: The study was performed in $\mathrm{NZ}$ rabbit papillary muscles ( $\mathrm{n}=48$; Krebs-Ringer: $1.8 \mathrm{mM} \mathrm{CaCl}_{2}, 35 \mathrm{C}$ ). Effects of selective ET-B activation by Sarafotoxin S6c (STRX; $0.2 \mathrm{mM} ; \mathrm{n}=6)$ and non-selective ET-A and ET-B activation by ET-1 ( $1 \mathrm{nM} ; \mathrm{n}=9)$ were analysed. Sarafotoxin S6c effects were also evaluated in the presence of NG-nitro-L-Arginine (L-NA; $90 \mathrm{mM}$; $\mathrm{n}=6$ ), Indomethacin (INDO; $1 \mathrm{mM} ; \mathrm{n}=6$ ) and in muscles without endocardial endothelium $(\mathrm{EE} ; n=6)$, while those of ET-1 were evaluated in the presence of a selective ET-A antagonist $(\mathrm{BQ}-123 ; 0.1 \mathrm{mM} ; \mathrm{n}=9$ ) or a selective ET-13 antagonist (BQ-788: $0.1 \mathrm{mM} ; \mathrm{n}=6$ ). Isotonic and isometric twitches were recorded and analysed. Reported parameters include: active tension (AT) and peak rate of tension development ( $\mathrm{dT} / \mathrm{d}$ max). Results (mean SEM. $p<0.05$ ) are expressed as \% change from baseline.

Results: SRTX-c induced a negative inotropic effect, reducing AT $(8.32 .1 \%)$ and $\mathrm{dT} / \mathrm{dtmax}(8.65 .6 \%)$. This effect was reversed by L-NA (AT increased $22.82 .9 \%$ and $\mathrm{dT} / \mathrm{dtmax} 20.12 .1 \%$ ), by INDO (AT increased $18.04 .4 \%$ and $\mathrm{dT} / \mathrm{dtmax} 18.94 .0 \%$ ) and by EE removal (AT increased 35.211.7\% and dT/dtmax 29.57.9\%). ET-1 increased $64.218 .2 \%$ AT and $58.620 .2 \% \mathrm{dT} / \mathrm{dtmax}$. This effect was enhanced by BQ-788 (AT increased $82.617 .5 \%$ and $\mathrm{dT} / \mathrm{dtmax}$ increased $121.326 .6 \%$ ) and reversed by BQ-123 (AT decreased $12.82 .7 \%$ and $\mathrm{dT} / \mathrm{dtmax}$ decreased $16.13 .0 \%$ ).

Conclusions: This study showed that stimulation of ET-B receptors elicits a negative inotropic effect, modulated by EE danage. This modulation seems to be mediated by $\mathrm{NO}$ and prostaglandins.

\section{0}

Endothelium-dependent vasodilation is impaired in down syndrome: a new target for therapy?

M. Cappelli-Bigazzi, G. Santoro, C. Battaglia, M.T. Palladino, M. Pascotto, C. Morelli, R. Calalr

Division of Pediatric Cardiology, "Monaldi" Hospital, Naples, I

Down's syndrome (DS) is characterized by an increased oxidative stress (OS) leading to degenerative pathologies due to oxygen free radical damage. This could be related to an over-expression of the enzyme $\mathrm{Cu} / \mathrm{Zn}$ superoxide dismutase whose gene is on the chromosome 21, with an increased generation of the more toxic $\mathrm{H}_{2} \mathrm{O}_{2}$. DS pts with congenital heart disease and systemic-to-pulmonary shunt show an increased pulmonary vascular (PV) reactivity with an higher incidence of $\mathrm{PV}$ obstructive disease. We hypothesize that this condition could be related to a reduced NO bioavailability secondary to abnormal OS. In 6 DS pts (age $79.5+39.1 \mathrm{~m}$ ) and 7 euploid pts $(65.3+22.3 \mathrm{~m} ; \mathrm{p}=\mathrm{NS})$, endotheliun dependent vasodilation with acetylcholine (ACH 0.1, 0.2 and $0.5 \mathrm{microg} / \mathrm{kg} / \mathrm{min}$ ) and endothelium independent vasodilation with nitroglycerin (NTG $0.2,0.5$ and $1.0 \mathrm{microg} / \mathrm{kg} / \mathrm{min}$ ) were studied. Brachial artery flow velocity was measured using an intravascular Doppler flow wire, and artery diameter was measured by quantitative angiography. DS pts showed a significant impairment of endothelial function compared 
with the controls. Responses to the 3 doses of $\mathrm{ACH}$ were, in fact, $16.0+5.5 \%, 33.7+11.0 \%$, and $62.6+15.4 \%$ in DS, whereas they were $29.4+5.2 \%(\mathrm{p}=\mathrm{NS}), 73.9+9.8 \%(\mathrm{p}<.05)$ and $107.8+$ $12.8 \%(\mathrm{p}<.15)$ in controls. Conversely, vasodilation to NTG was not different between the two groups, being $31.3+11.2 \%, 56.4+$ $11.9 \%$ and $70.1+11.9 \%$ in DS, and $35.7+11.0 \%$ (p = NS), $60.2+8.5 \%(\mathrm{p}=\mathrm{NS})$ and $78.3+10.1$ in controls.

In conclusion, our study suggests that DS subjects have an impaired endothelial function in childhood. It could be hypothesized that this impairment might be due to increased oxidative stress. If our hypothesis will be confirmed by further studies, new prophylactic and therapeutic perspectives for pulmonary vascular obstructive disease in DS subjects might be available.

\section{1}

Beneficial effect of steroids on cardiac function of patients with Duchenne muscular dystrophy

A. Fournier, J. Thrien, M. Filiatrult, Y. Brousseau, M. Vanasse

Ste-Justine Hospital, Moutréal, CDN

Long term steroids (S) have been proven efficacious in maintaining muscular strength in boys with Duchenne muscular dystrophy (DMD). Significant side effects such as hypertension, obesity, short stature, cataracts, osteoporosis are encountered. The effect of such treatment on cardiac function, a major determinant in the outcome of these patients (pts), has never been reported.

A total of 86 pts with IDMI) were studied. Echocardiographic parameters were compared in 38 boys on $\mathrm{S}$ (Deflazacort) for a period ranging from 3 to 7 years to 48 boys not treated with $S$. At time of evaluation age of pts was comparable, pts receiving S 13.3 t.0 years ws $1+.53 .8$ years in the others. Shortening fraction (SF) and ejection fraction were higher in pts receiving $\mathrm{S} 31.05 \%$ vs 27 6\%: $536 \%$ vs $4610 \%, \mathrm{p}<0.05$. Dilated cardiomyopathy defined as a SF $<28 \%$ and/or LV $>95$ th percentile for height was present in $12 / 38(32 \%)$ pts receiving $S$ vs $30 / 48(63 \%)$ in the others, $p<0.15$. Systolic blood pressure expressed as $Z$ score was not significantly higher in pts receiving $S, 0.250 .91$ vs -0.031 .19 , $\mathrm{p}=0.30$ but ACE inhibitors were given in $19 / 38(50 \%)$ pts receiving $S$ vs $11 / 48(23 \%)$ in the others

In conclusion, pts with DMD) treated with $\mathrm{S}$ have a better cardiac function than pts not receiving $S$. These findings add another argument to pursue $S$ in thesc pts even if side effects are encountered while waiting for a definite genetic treatment.

\section{2}

Neonatal screening for critical congenital

heart defects - low sensitivity for lesions with

duct-dependent systemic circulation

J.Sunnegardh, .M.Mellander

The Queen Silvia Childrens Hospital, Göteborg University, Göteborg, S

Although early detection of duct-dependent congenital heart disease is a prerequisite for survival and optimal treatment, there is a lack of data on the sensitivity of neonatal screening for such defects in Sweden

Material and method. Data was retrieved retrospectively in infants with immediately life threatening heart defects operated before two months of age in our institution from 1993 to 2001. During this time period there were 351843 births in our referral area. Results. 293 patients fulfilled the inclusion criteria. Forty-nine $(16,7 \%)$ with birth weights $<2500 \mathrm{~g}$ and/or gestational age $<37$ weeks were excluded. Duct-dependent systemic circulation was present in $129(52 \%)$ infants. duct-dependent pulmonary circulation in $96(38 \%)$ and in $2+$ patients $(10 \%)$ the defect was not duct-dependent. Median age and range at suspicion of heart disease was 1 day $(0-45)$ in all children, in infants with ductdependent pulmonary circulation $<1$ day $(0-13)$, in children with duct-dependent systemic circulation 2 days $(0-45)$ and in children with no duct-dependent circulation 3,5 days $(0-42)(p<0.001)$. In 51 infants $(20 \%)$ the heart defect was not suspected before discharge from the maternity ward. Such late detection occurred more often in children with duct-dependent systemic circulation $(30 \%)$ and in children without duct-dependent defects $(50 \%)$ as compared to children with duct-dependent pulmonary circulation $(3 \%)(\mathrm{p}<0.001)$. The proportion detected after discharge increased from $13 \%$ in $1993-1995$ and $20 \%$ in $1996-1998$ to $29 \%$ in 1999-2001 ( $p=0.013)$. No difference was found in postoperative mortality comparing children diagnosed before or after discharge from the maternity ward ( $5 \%$ vs $3.9 \%$ )

Conclusion. The proportion of infants with critical congenital heart defects diagnosed after discharge from the neonatal maternity ward is unacceptably high and has increased in recent years The true incidence is even higher as children with late diagnosis who died at home or who did not receive surgery as a consequence of late diagnosis (brain damage) were not included in this study. Neonatal screening failed mainly in children with ductdependent systemic circulation. The worsening results are probably explained by the extended use of early discharge post partum in combination with other changes of maternity ward routines.

\section{3}

Persistent ductus arteriosus and infective endocarditis - a forgotten entity

M. Sadiq, F. Latif, M. Nazir, S.A. Sheikh

Punjab Institute of Cardiology \& Childrens' Hospital, Lahore, Pak

Background: Once a serious complication, infective endocarditis (IE) has become extremely uncommon in patients (pts) with persistent ductus arteriosus (PDA). Early diagnosis \& closure has essentially made it an unheard entity in the West. The only indication of closure of a small PDA being prevention of SBE is often debated.

Objective: To assess the incidence, pattern $\&$ outcome of IE in PDA in an ongoing study on IE in children.

Setting: A tertiary referral center for paediatric \& adult cardiology. Patients \& Methods: All children with IE in PDA admitted to a single center over a period of six-years (April 95-March 01) were analysed. The diagnosis was based on Dukes criteria, which proposed 2 major and 6 minor criteria. Minor criteria were expanded to include raised acute phase reactants and newly diagnosed or increasing splenomegally. PDA were classified as small, moderate and large by standard methods.

Results: Of 2908 hospital admissions, PDA was the major underlying lesion in 368 pts (12.6\%). Of all hospital admissions, 96 fulfilled the diagnostic criteria for IE. Of these, PDA was the underlying lesion in 12 pts $(12.5 \%)$. The mean age was $8.9+4$ years with only one patient under one year of age. Seven patients had small PDA while 3 had moderate and two large. Blood cultures were positive in 5 pts $(42 \%)$ while vegetations on echocardiography were present in 10 pts $(83 \%)$. The duration of treatment was $4-6$ weeks in all but one patient, who needed 10 weeks antibiotics. Surgery for IE was not required in any patient. All 12 patients had subsequent closure, 8 by occlusion devices (Coils, Amplatzer device and Rashkind's umbrella) and 4 by surgery.

Conclusions: The incidence of IE on PDA is $0.4 / 1000$ hospital admissions in a tertiary paediatric cardiology referral center. Majority are small PDA's and an early closure irrespective of the size of duct may have prevented this serious complication in majority of pts. 
44

\section{Histopathological evaluation on late myocardial changes following Kawasaki disease with coronary aneurysm using endomyocardial biopsy}

S.Yonesaka, T. Takahashi, T.Sato, K.Otani, S. Eto, K. Ichinose

Hirosaki University, Hirosaki,J

Purpose: To investigate the late myocardial changes and the relationship between coronary artery lesions (CAL) and myocardial damages, serial coronary arteriography and endomyocardial biopsy (EMB) were performed in the patients with Kawasaki disease (KD), followed up more than ten years after onset.

Patients and method: They included 12 patients with giant coronary aneurysm (G-AN); the male to female ratio was $7: 5$ and 16 patients with CAL; the male to female ratio was 9:7. Age at initial study was 6 months to 12 years. All patients were followed up more than ten years (11 to 20 years) after onset. Epicardial coronary arterial lesions were analyzed semiquantatively with MIPRON and histopathology was evaluated with histomorphometric method to calculate the percent area of myocytes, fibrous tissue and fatty tissue and small vessel changes.

Results: Initial vs. remote myocardial changes on EMB, such as fibrosis, degeneration, disarray and inflammatory cell infiltration, showed $62 \%$ vs. $57 \%, 54 \%$ vs. $38 \%, 32 \%$ vs. $65 \%$ and $23 \%$ vs. $26 \%$, respectively in the patients with G-AN, while $25 \%$ vs. $54 \%, 33 \%$ vs. $38 \%, 50 \%$ vs. $38 \%$ and $17 \%$ vs. $0 \%$, respectively in the KD patients with CAL. In one of the KD patients with CAL, massive inflammatory cell infiltration and myocytolysis was found in the subsequent study, which suggested chronic myocarditis.

Conclusion: Myocardial changes in the patients with G-AN were relatively mild on light microscope but still remained and ultrastractural changes as microangiopathy in late stage. Some cases of KD may develop chronic myocarditis leading to a cardiomyopathy-like state. Further follow-up study should be required to clarify the significance of the myocardial sequelae of $\mathrm{KD}$ with close attention not only to CAL but also to the myocardial changes in long-standing cases of the disease.

\section{Session 6: Short Abstract Presentations 2} 45

The new intratherapeutics doublestrut stent: in-vitro evaluation of stent geometry following overdilation and initial clinical experience in congenital heart disease F. Ing, J. Mathewson, M. Cocalis, S. Kirkpatrick, K. Maginot, J. Perry Children's Hospital of San Diego, San Diego, USA

Background: The new IntraTherapeutics Doublestrut stent (DS) is FDA-approved for biliary use, but was found to have features which may benefit congenital heart disease (CHD) pts. It has an "open-cell system" designed for greater flexibility and can be dilated to $12 \mathrm{~mm}$ diameter, but preliminary studies suggest that it can be overdilated to $18 \mathrm{~mm}$.

Methods: In order to determine if this stent is useful in CHD (dilatable to $18 \mathrm{~mm}$ diameter), in-vitro evaluation was first performed by serially overdilating the DS to $18 \mathrm{~mm}$ diameter. Stent dimensions and cell geometry were evaluated. Clinical trials were started after benchwork testing demonstrated stent expansion up to $18 \mathrm{~mm}$ diameter. Cath lab data were evaluated.

Results: In-vitro testing demonstrated $26-33 \%$ foreshortening when the $26 \mathrm{~mm}$ DS was expanded directly to $18 \mathrm{~mm}$ diameter. However, when serially dilated with progressively larger balloons up to $18 \mathrm{~mm}$, stent foreshortening was not noted due to change in the geometry of each cell. Stent length was maintained by length-wise separation between the rows of "open-cells". Between 4/00 and 10/01,22 pts with CHD underwent implantation of 33 DS. The median age and wt of the pts were $1.6 \mathrm{yr}$ and $10.2 \mathrm{~kg}$ respectively. All stents were implanted with $6-15 \mathrm{~mm}$ diameter balloons via $7-9 \mathrm{Fr}$ sheaths. 32 of 33 stents were implanted successfully. The mean gradient decreased from $27.413 .5 \mathrm{mmHg}$ to $5.65 .3 \mathrm{mmHg}$ and mean minimum diameter increased from $4.72 .5 \mathrm{~mm}$ to $8.73 .3 \mathrm{~mm}(\mathrm{p}<0.05)$. The right ventricle to femoral artery pressure ratio decreased from $65.65 .7 \%$ to $47.911 .8 \%(\mathrm{p}<0.05)$. One stent was partially dilated before circumferential balloon rupture resulting in surgical removal and PAplasty. In the OR, it was felt that the spicules on the heavily calcified homograph rather than the stent was the culprit. Other complications included 4 pinhole balloon ruptures at the end of inflation after the stent was fully expanded. Careful inspection of the angiograms indicated that stent length did not have measureable foreshortening in all cases.

Conclusion: The new DS can be overdilated to $18 \mathrm{~mm}$ diameter Foreshortening was not seen when dilated up to $15 \mathrm{~mm}$ in-vivo and serially up to $18 \mathrm{~mm}$ in-vitro. This $\mathrm{S}$ can be used safely and effectively to treat pts with CHD.

\section{6}

Anti-Ro and anti-La antibodies and their effect on conduction in the absence of overt heart block - a prospective study

P. Gordon, M. Khamashta, G.R.V. Hughes, E. Rosenthal,

J. Simpson, G. Sharland

Guy's and St. Thomas' Hospital, London, UK

Background: Congenital complete heart block occurs in $2 \%$ of fetuses whose mothers are anti-Ro/SSA antibody positive with a recurrence rate of $16 \%$. In animal models varying degrees of congenital heart block have been observed.

Aim: To evaluate prospectively the effect of congenital exposure of the anti-Ro or La antibody on the conduction in the human. Methods: Women with connective tissue disease were recruited at 20 weeks gestation. Anti-Ro and anti-La antibody status were determined by line immunoassay (INNO-LIA ANA Update). ECGs were performed on the child at the postnatal follow up. ECGs were analysed using a vernier caliper (Draper) by a single blinded observer. RR, PR, QRS were measured on three successive beats. Measurements were compared between the anti-Ro or $\mathrm{La}$ antibody positive ( $\mathrm{Ro}^{+}$) group and anti-Ro and La negative (Ro - ve) groups using the t-test.

Results: To date 56 women have participated in the study, 32 (Ro $+v e$ ), and 24 (Ro - ve). Two women had twin pregnancies ( 1 Ro +ve, 1 Ro - ve). No ECG was available in 6 cases, one due to fetal death (Ro + ve, IUGR) and two due to neonatal death (both Ro +ve, no heart block). Three dropped out of the study (2 Ro +ve, $1 \mathrm{Ro}-\mathrm{ve}$ ) but had no overt heartblock. In addition one child developed CCHB (Ro + ve). ECGs were performed in the remaining 51 children postnatally (median 8 weeks, SD 3.59) 27 were born to Ro positive, 24 to Ro - ve mothers. There was no significant difference in RR, PR, QRS between the two groups. Conclusion: In the absence of overt conduction defects, the antiRo antibody has no effect on conduction as measured on ECG suggesting that the effects on anti-Ro antibodies may be "all or none".

\section{7}

Implantation of large Amplatzer devices to close atrial septal defects in children: is it a safe practice?

M.J. Raboisson, M. Bourdages, E. Piette, P. De Guise, M. Brassard, J. Mir Hôpital Sainte Justine, Montréal, CDN

Background and objectives: Concerns have been expressed that devices with large disc diameters and profile, such as the Amplatzer, 
night distort, obstruct or compress various vital structures. These oncerns are particularly justified in children, in whom large levices might interfere with the normal growth of structures such $s$ the A-V annuli or the aortic root. We thus aimed at verifying the hort and mid-term tolerance of large Amplatzer devices implanted $\mathrm{n}$ patients with uncompleted growth.

'opulation and method: From 12/97 to 06/01, 80 patients $<12$ yrs mderwent percutaneous closure of an ASD with the Amplatzer levice. The 20 patients with the largest devices (size of left atrial lisc/size of patient $0,0370,003)$ were reviewed clinically, echocarliographically and electrocardiographically. Mean age, weight and 2p/Qs were 4,681,98 yrs, $15,23,6 \mathrm{~kg}$ and 2,71,0 respectively. The ISD diameter and device size were $17,44.5 \mathrm{~mm}$ and $23,43,3 \mathrm{~mm}$. Zesults: No procedural complication was observed. The short erm follow-up was complicated by $\mathrm{A}-\mathrm{V}$ conduction disturbances $\mathrm{n}=3)$, pericardial effusion $(\mathrm{n}=5)$ and moderate mitral regurgiation $(\mathbf{n}=1)$. All these complications produced no symptoms ind spontaneously recovered by one month. At last follow-up $13,210,2$ months) there was no case of increased valvar regurgitaion (compared to pre-implantation), systemic or pulmonary renous obstruction, significant rhythm or conduction distursances, progressive aortic root distortion or any other complicaion. At immediate follow-up, proto-systolic contact between the eft disc and the anterior mitral leaflet and straddling of the aortic oot by the device were respectively observed in $50 \%$ and $60 \%$ of jatients. By last follow-up, the prevalence of such anomalies was $20 \%$ and $45 \%$, while the mean distance between the left atrial disc and the plane of the mitral annulus increased from $0,92,2 \mathrm{~mm}$ to $2,12,0 \mathrm{~mm}(\mathrm{p}<0,01)$ and the amount of aortic straddling decreased from $2,12,6 \mathrm{~mm}$ to $0,82,3 \mathrm{~mm}(\mathrm{p}<0,01)$.

Results: Large Amplatzer devices produce an increased amount of mmediate complications, which were all asymptomatic and sponaneously recovered in our series. These devices do not seem to zause mid-term complications or progressive distortion of cardiac structures, even in a population of growing children, confirming the safety of this practice.

48

Thrombus formation in patients after Fontan operation detected by transoesophageal echocardiography G. Balling, M. Vogt, H. Kaeminerer, A. Eicken, R. Lange, J. Hess Deutsches Herzzentrum München, München, $D$

Objectives. Despite of any data from a prospective randomized study to adress the issue of anticoagulation versus no routine anticoagulation in patients after a Fontan operation we continued our investigation about intracardiac thrombus formation detected by routine transoesophageal echocardiography ${ }^{\star}$

Methods. We evaluated 99 patients (46 female, 53 male) who had undergone a Fontan-type operation (RA-PA: 39, RA-RV: 27, TCPC: 30 , others: 3 ), using transesophageal echocardiography $(\mathrm{n}=150)$. The patients age at investigation ranged between 1,7 and 41.7 years (median 18,3 years). The age at the definitive Fontan procedure varied between 0,5 and 34 years (median 7,2 years). The mean interval of observation time since the Fontan operation and our study ranged from $0.2-20.4$ years (median 9.9 years).

Results. In 23 patients (23\%; CI 95\%:15,2-32,5\%) a thrombus formation could be found. Neither underlying morphologic disease, nor age at operation, type of Fontan-operation, sex, follow-up interval, arrhythmias, laboratory or hemodynamic findings could be identified as predisposing risk factors.

Conclusion. Because of the high incidence of thrombi we recommend annual transesophageal studies and a life-long oral anticoagulation therapy in all patients after a Fontan procedure.

( $\star$ J Thorac Cardiovasc Surg 2000; 119: 745-52).
49

Spiral CT scan and angiographic correlations of stents implanted in the aortic arch

M. Chessa, M. Carminati, G. Butera, R.M. Bini, M. Drago,

F. Voterrani, F. Bedogni, D. Negura

Istituto Policlinico San Donato, S. Donato Milanese (MI), I

Background: Stent treatment for coarctation of aorta has shown excellent immediate results. However, continuous clinical and image-derived follow-up are required to evaluate possible recurrent stenosis and/or complications as aneurysm formation. The aim of this study was to evaluate the mid-term follow-up results by means of angiography and spiral CT scan.

Methods: Up to December 200128 patients $(9$ pts with native coarctation, mean age at treatment $21,213,6$ yrs; 19 pts with recoarctation, mean age at treatment $16,827 \mathrm{yrs}$ ) underwent stent implantation in the aortic arch. Immediately after stent implantation mean systolic pressure gradient dropped from $39,617.5 \mathrm{mmHg}$ at $34,7 \mathrm{mmHg}$.

All patients underwent echocardiographic, angiographic and spiral CT scan evaluation at the mean clinical follow-up time of 1913.5 months. All patients remain normotensive and symptoms free.

Results: There were no significant changes in systolic pressure gradient $(54,3 \mathrm{mmHg}, \mathrm{p}>0.05)$. No late aneurysm or restenosis were observed. The spiral $C T$ scan visualized stent position, and structure with very good quality images and 3-D reconstruction. Conclusion: Stent repair of aortic coarctation provides excellent immediate and mid-term follow-up results. The spiral CT scan is safe, easy and can be performed several times providing a perfect morphological follow-up.

\section{0}

Influence of hypoxaemia on the function of ATP dependent $\mathrm{K}+$-channels in cardiomyocytes from children with congenital cardiac defect

K. Schumacher, E. Jungling, J.F. Vazquez-Jimenez, G. von Bernuth, M-C. Seghaye, A. Luckhoff

Institute of Physiology, University Aachen, Germany, München, D

Background: ATP-dependent potassium (Katp) channel are thought to play an important role in cardiac protection against tissue hypoxia.

Objectives: To investigate the effect of chronic hypoxia on the function of Katp channels in atrial cardiomyocytes taken from children with congential cardiac defect.

Methods: 6 children (1-59 months) with cyanotic congenital cardiac defect were investigated and 6 adult patients (age: 52-74) with coronary disease served as controls. Cardionyocytes were isolated from atrial samples obtained before institution of extracorporeal circulation. Katp channels were activated by the potassium channel opener pinacidil and inhibited by the sulfonylurea glibenclamide. Katp currents were measured by patch clamp technique using the conventional whole cell mode.

Results: In cells obtained from adult patients, pinacidil evoked a significantly increase of Katp current from $1.390 .17 \mathrm{pA} / \mathrm{pF}$ (mean $\mathrm{SEM})$ to $22.63 .43(\mathrm{p}<0.01)$. In cells obtained from children, pinacidil also evoked an increase of Katp current from 0.730 .12 $\mathrm{pA} / \mathrm{pF}$ to $4.741 .08 \mathrm{pA} / \mathrm{pF}(\mathrm{p}<0.05)$ but this increase was significantly lower than in adult cells $(\mathrm{p}<0.05)$. In both groups, glibenclamide led to the return of Katp current to baseline levels. Conclusion: These results show for the first time that chronic hypoxaemia related to congenital cardiac defect leads to decreased function of Katp channels. This could be the result of the exhaustion of these channels by chronic hypoxaemia and could imply 
reduced endogenous protective mechanisms in the nyocardium of children with cyanotic cardiac defect.

\section{1}

Prevalance and spectrum of cardiac abnormalities in pediatric patients with intestinal malrotation

E. Thomas, A.P. Salmon, B.R. Kecton, J.P. Gnanapragasant,

J.J. Vettukattil

Southampton General Hospital, Southampton, $U K$

Isomerism of the atrial appendages is well known to be associated with malrotation of the gut and complications of intestinal volvulus. However the prevalence association of congenital cardiac defects in this group of patients is not well documented.

We retrospectively analysed the casenotes of the 37 pediatric patients (21 males) age range 1 day to 9.5 years (mean 1.44 years, mode 1 month) who had undergone Ladd's procedure for intestinal malrotation in our institution during the 5 year period from $3 / 96$ to $5 / 00.21(56.7 \%)$ were operated upon in the neonatal period. In 12 patients $(32.4 \%)$ the initial presentation was with volvulus involving the small intestine. $23(62.2 \%)$ had a primary diagnosis of malrotation of the gut. Other associated surgical defects were Meckel's diverticulum (3), saddle liver (3), asplenia (2), right descending colon (1), abdominal situs ambiguus (1), horse-shoe kidney (1), pyloric stenosis (1), appendix abscess (1), dysplastic right kidney (1). Only $10(27 \%)$ of the 37 patients had a cardiac review at the time of surgery. In only one $(2.7 \%)$ the cardiac evaluation was initiated by the pediatric surgeons; in $5(13.5 \%)$ it was requested by the neonatologist or general pediatrician and $3(8.1 \%)$ were referred to the surgeons by the pediatric cardiologist.

The primary cardiac diagnosis amongst the 10 patients reviewed cardiologically was right atrial isomerism (3), left atrial isomerism (3), supracardiac total anomalous pulmonary venous connection (1), scimitar syndrome (1) and hypertrophic obstructive cardiomyopathy (1).

This study thus underscores the importance of cardiac assessment of any pediatric patient presenting with intestinal malrotation. There should also be a low threshold for entertaining the possibility of malrotation of the gut in congenital heart disease patients with GI symptoms, particularly those with suspected or confirmed atrial isomerism.

\section{2}

\section{Interventional catheterization procedures in the} treatment of hypoplastic left heart syndrome

A. Uebing, G. Fischer, J. Stich, J.Scheewe, A. Boening, H.H. Kraner

Department of Paediatric Cardiology, Lniversity of Kiel, Kiel, D

Interventional catheterization procedures are an important constituent of the therapy of hypoplastic left heart syndrome (HLHS) in the context of the three stage surgical palliation.

Since 1996, 71 patients underwent stage one Norwood procedure (N I) (survival rate $85 \%$ ). In 10 of 54 patients who were examined by cardiac catheterization aortic coarctation occurred and was treated by balloon angioplasty. In all patients the initial intervention was successful with a decrease in the pressure gradient from $30.3 \pm 13.2$ to $3.0 \pm 4.0 \mathrm{mmHg}(\mathrm{P}<0.0001)$. In 5 patients a recoarctation occurred and was less successfully treated by balloon angioplasty at a median of 4.6 months after initial intervention (reduction of pressure gradient: $28.3 \pm 8.1$ to $19.5 \pm 7.6 \mathrm{mmHg}$; NS). In 3 patients a surgical enlargement of the aorta had to be performed as part of subsequent operation.

As second stage of the surgical palliation a hemi-Fontan procedure (hFP) was performed in 52 patients (survival rate $94 \%$ ). 32 of 52 patients were examined by cardiac catheterization. Aortopulmonary collateral blood flow could be diagnosed in all patients. Most collateral vessels originated from right sided arteries perfusing parts of the right lung (79\%). In $24 / 32$ patients coil embolisation of collateral vessels was considered to be necessary and successfully performed.

The fenestrated Fontan procedure (fFP) was performed in 18 patients (survival rate $89 \%$ ). In 7 of 10 patients who were examined yet the fenestration was successfully occluded using an "Amplatzer Septal Occluder" ( $\mathbf{n}=5)$, Rashkind-occluder $(\mathbf{n}=1)$ or coil $(\mathrm{n}=1)$. After occlusion arterial oxygen saturation increased $(87.4 \pm 3.4 \%$ vs. $95.3 \pm 1.5 \% ; \mathrm{P}<0.0101)$ whereas central venous pressure remained unchanged $(12.7 \pm 1.4 \mathrm{mmHg}$ vs. $13.6 \pm 1.8$; NS). In 3/10 patients the baffle fenestration occluded spontaneously. Additional interventional procedures in the management of HLHS were coil embolisation of interatrial communications producing right to left shunt via small cardiac ("thebesian") veins $(\mathrm{n}=2$ after hFP and $\mathrm{n}=2$ after fFP) and coil enbolisation of veno-venous collateral vessels $(n=4$ after $f F P$ ).

After N I the most frequent intervention is the balloon angioplasty of aortic coarctation. Coil embolisation of collateral vessels is mostly necessary after hFP. Closure of the atrial baffle fenestration is the final step in the combined surgical and interventional treatment of HLHS.

\section{Session 7: General}

53

Longterm neurodevelopmental outcome after neonatal arterial switch operation (ASO) for transposition of the great arteries (TGA)

H.H. Hvels-Grich, R. Schnitker, K. Konrad, M. Wiesner,

M.-C. Seghaye, W. Huber, F. Kotlarek, B.J. Messmer, G. von Bernuth Department of Paediatric Cardiology, Univ.Hosp, RUTH Aachen, Aachen, D

The purpose of this prospective longitudinal study was to assess neurodevelopmental status of children between 8 and 14 years of age after neonatal ASO for TGA.

60 unselected children operated with combined deep hypothermic circulatory arrest (DHCA) and low flow cardiopulmonary bypass (CPB) underwent, at mean age 10.5 1.6 (mSTD), standardized reevaluation of neurological status, gross motor function. intelligence, academic achievement, language and speech. Results were related to pre-, peri-, and postoperative status and management as well as to neurodevelopmental status at midterm followup (mean age 5.4 years).

Neurological and speech impairment were more frequent $(27 \%$ and $40 \%$, respectively) than in the normal population. Compared to normal children, formal intelligence and socioeconomic status were not different, while motor function, acadenic achievement and expressive language were significantly reduced $(p<0.04$ each). Overall rate of developmental impairment in one or more domains was $55 \%$ compared to $26 \%$ at age 5.4 years. Multivariable analysis showed that severe preoperative hypoxia predicted reduced motor function (mean deficit 52.7 points, $p<0.001$ ). Longer bypass duration predicted both neurological and speech dysfunction (Odds ratio $1.8, \mathrm{p}=0.04$ and $1.9, \mathrm{p}=0.02$, per 10 minutes of bypass duration). Peri- and postoperative cardiocirculatory insufficiency predicted neurological (Odds ratio 6.5, $p=0.04$ ) and motor dysfunction (mean defict 6.8 points, $\mathrm{p}=0.03$ ). Low socioeconomic status was predictive for dysfunction of intelligence (mean deficit 17.3 points, $\mathrm{p}=0.001$ ), academic achievement (mean deficit 17.2 points, $\mathrm{p}<0.001$ ), expressive and receptive 
language (mean deficit 8.9 points, $p<0.01$ and 14.6 points, $\mathrm{p}<0.001$, respectively).

The neonatal ASO with combined DHCA and low flow CPB is, increasing with age, associated with reduced neurodevelopmental outcome, but not with cognitive dysfunction as based on formal intelligence testing. The risk of longterm neurodevelopmental impairment after neonatal corrective cardiac surgery is related to deleterious effects of the global perioperative management as well as to special adverse effects of prolonged bypass duration. Severe preoperative hypoxia and postoperative hemodynamic instability must be considered as important additional risk factors.

54

Comparison of health related quality of life

(MOS SF-36) with exercise testing in adolescents and adults with congenital heart disease

A. Hager, J. Hess

Deutsches Herzzentrum München, TU München, München, D

Objective: To compare the self estimation of the exercise capacity by a questionnaire with the objective exercise test performance in patients with congenital heart disease.

Patients and methods: 29 patients ( 15 female, 14 male, $12-58$ years old) with various congenital heart defects answered a health related quality of life questionnaire (MOS SF-36). Then they performed a cardiopulmonary exercise test with measurement of $\mathrm{O} 2$ uptake (VO2) on a bicycle in sitting position.

Results: VO2max showed better correlation with the subjective estimation of exercise capacity than VO2 at anaerobic threshold and both VO2 measurements normalized to the expected VO2max. There was a significant correlation with the physical scale $(r=0.417, p>0.05)$, but not with the psychological scale of quality of life $(\mathrm{r}=-0.106$, n.s.). Analysis of detailed subscales showed a highly significant correlation to 'physical function' $(\mathrm{r}=0.663, \mathrm{p}>0.001)$. No significant correlation could be found to the subscales of 'general health', 'physical role', 'emotional role', 'social function', 'pain', 'vitality', and 'mental health'.

Conclusions: Patients with congenital heart defects can reliably self estimate their exercise capacity. A standardized pretest questionnaire can help to find an adequate protocol for exercise testing. Correlation of exercise performance to other subscales of quality of life especially to the psychological ones is poor.

\section{5}

Neuropsychological and neurodevelopmental outcome of children with hypoplastic left heart syndrome following the three-stage Norwood Procedure: a British Experience

J. Blyth, E. Wassmer, W. Braun, J. Wright, C. Jones, C. Penrose Clinical Psychology, Birmingham Children's Hospital, Birmingam, UK

Introduction: Approximately 200 children with Hypoplastic Left Heart Syndrome (HLHS) are born in the UK per annum. The majority are managed with terminal supportive care. Improved survival rates following palliation has led to an interest in quantifying the impact of surgery on intellectual functioning and quality of life. Method: Eleven children with HLHS post Fontan repair were assessed. Data relating to possible predictors of neurological and cognitive outcome were retrieved from medical notes. A neurological examination was performed including the Functional Independence Measure (WeeFIM). Cognitive functioning was assessed using Wechsler age appropriate Intelligence tests. Siblings were available to be used as controls in 9 cases and underwent the same neurological and psychometric testing.
Results: 11 children with HLHS were assessed (mean age 5.63 years, range 4.03-7.21). Only one child, had a WeeFIM score that was lower than expected suggesting a mild functional impairment. Nine siblings were assessed (mean age 6.76 years, range 4.72-9.30). One had lateral nystagmus (sib of above), all had WeeFIM scores suggesting normal function capacity. Differences in verbal, performance and full-scale IQ between HLHS child and corresponding sibling were compared using a paired t-test. A significant trend was observed for full-scale $\mathrm{IQ}(\mathrm{p}=0.011)$. The mean full-scale IQ for the siblings was 98.55 ( $\mathrm{SD}=18.22$ ), and for the HLHS children $85.11(\mathrm{SD}=11.98)$. A significant trend was observed for verbal IQ $(p=0.017)$. The mean verbal IQ for siblings was $98.11(\mathrm{SD}=16.03)$ and for the HLHS children 82.44 $(\mathrm{SD})=13.92)$. No significant difference was observed for performance IQ $(p=0.102)$. Regression analysis reveals that the presence of clinical seizures is negatively predictive of full-scale IQ. Two of the eleven HLHS children had seizures. Their mean full-scale IQ was 77.0 (SD 1.41); the remainder of the HLHS children had a collective mean full-scale IQ of 90.78 (SD 12.92). This is statistically significant $(\mathrm{p}=0.013)$.

Conclusion: The mean full-scale IQ for HLHS children in our cohort is similar to that reported by other investigators. However, we found a significant difference between the full-scale IQ of HLHS children post Fontan and their corresponding siblings. Nevertheless, physical and functional prognosis following palliation in HLHS is good.

\section{6}

Modified Norwood procedure and subsequent cavopulmonary anastomoses for classic hypoplastic left heart syndrome (classic HLHS): short-term and intermediate results

B. Nilsson, M. Mellander, T. Gilljam, G. Sdow, H. Berggren

The Queen Silvia Childrens Hospital, Göteborg, S

We evaluated our first 9 years experience of staged surgical palliation for classic HLHS.

Material and methods: From May 1993 to December 2001, 54 neonates with classic HLHS, defined as mitral atresia and/or aortic atresia with hypoplasia of the ascending aorta, and absence or severe hypoplasia of the left ventricle, were admitted to our institution. Twelve patients were not operated ( 7 parental refusal, 2 extracardiac malformations, 2 preterm, 1 ascending aorta $<1 \mathrm{~mm}$ ). Preoperative right ventricular function and tricuspid regurgitation were graded from 2D echo and colour doppler. Other risk factors evaluated included gestational age, age and weight at surgery, preoperative ventilatory support, resuscitation and acidosis, ascending aortic diameter, shunt cross-sectional area to body surface area ratio, time on deep hypothermic circulatory arrest (DHCA) and extracorporeal circulation (ECC). Risk factors for death after stage I (until stage II) were analysed using Coxs proportional hazard regression modeling.

Results: Forty-two patients underwent modified Norwood procedure (stage 1), at a median age of 7 days (range 1 to 19). Hospital survival was $31 / 42(74 \%)$, with 7 late deaths (before stage II). Time on DHCA and ECC were the only significant risk factors for death after stage $I$ ( $p=0.009$, and $p=0.007$, respectively). The last 8 patients were operated in moderate hypothermia without circulatory arrest. Two died before stage II compared to 16 of 34 operated with DHCA (n.s.). Twenty-four patients underwent bidirectional cavopulmonary anastomosis, at a median age of 6.5 months (range 2.0 to 9.5), with 5 late deaths, and one patient had a heart transplant. Nine patients underwent total cavopulmonary connection (TCPC), at a median age of 24.6 months (range 
21.2 to 41 ), with no mortality. Kaplan-Meier survival was $55 \%$, $47 \%$, and $44 \%$ after 6 and 12 months, and 4 years, respectively. Conclusion: These results refer to a complete series of Norwood surgery for classic HLHS and thus includes the initial learning curve. The main mortality occurred after stage I. Time on DHCA and ECC were significant risk factors for stage I mortality. The results in the last 8 patients operated without DHCA encourage us to further evaluate this technique.

\section{7}

Ascending aortic growth following Norwood operation

B.H.P. Nagel, M. Chaudhari ${ }^{\star}$ J.G.C. Wright*, D. Barron $\star$, W.J. Brawn*, O. Stumper*

University Children's Hospital, Essen, D. \& Birmingham, UK

Objective: To investigate the pattern of relative growth of the ascending aorta (AAO), descending aorta (DAO) and pulmonary artery (PA), in patients with hypoplastic left heart syndrome (HLHS) after Norwood palliation.

Methods: 25 randomly selected patients with HLHS after Fontan operation were investigated. Aortic atresia (AA) was present in 17 $(68 \%)$ and aortic stenosis in $8(32 \%)$. Serial angiographic measurements of AAO, DAO and PA were performed. Results at first cath prior to cavopulmonary shunt (age $0.39(0.15-1.39)$ years), and final assessment prior to Fontan operation $(3.5(2.1-4.7)$ years) were compared. Age at assessment did not differ significantly between the 2 groups. Ratios of AAO/PA, PA/DAO and AAO/DAO were calculated to derive age and weight independent values. Statistical analysis was performed using student $t$-test for paired samples.

Results: In AA patients mean values for $\mathrm{AAO} / \mathrm{PA}$ and $\mathrm{AAO} / \mathrm{DAO}$ ratios decreased between first $(0.28$ (SD 0.08) and 0.54 (SD 0.01)) and second $(0.23(0.05)$ and $0.47(0.13))$ assessment $(\mathrm{p}<0.01)$. $\mathrm{PA} / \mathrm{DAO}$ ratios remained unchanged (first: $2.0(0.27)$; second: $2.07(0.35)$ ). In AS patients AAO/PA (first: $0.46(0.13)$; second: $0.49(0.08)$ ), AAO/DAO (first: $0.92(0.19)$; second: $0.96(0.17)$ ) and PA/DAO ratios (first: 2.09 (0.57); second: $2.02(0.4)$ ) did not change significantly with time. AAO/PA and AAO/DAO ratios were significantly higher at first and second assessment in AS patients compared to AA patients $(p<0.0005)$.

Conclusions: Growth of the ascending aorta in patients with aortic stenosis is preserved, whereas it is reduced in patients with aortic atresia. This may have an impact on coronary perfusion and thus long term right ventricular performance.

\section{8}

Complete atrioventricular septal defect (CAVSD): outcome in relation to $\mathrm{LV}$ size

G. Ciotti, R. Pande, R. Holzer, M. Pozzi

Royal Liverpool Children's Hospital, Liverpool, UK

Aim: Study of prognosis of biventricular and univentricular repair of CAVSD.

Population: Since 1992 in our centre the same surgeon operated on 140 patients (pts) with CAVSD.

I group: 67pts (47.9\%), balanced simple CAVSD associated with minor cardiac defects (multiple VSD's, double orifice MV). The other 73pts (52.1\%) had unbalanced or complex CAVSD and were subdivided in three groups in relation of their surgical correction: II group univentricular repair: 40pts (54.8\%) with hypoplastic LV: 33pts (82.5\%) or hypoplastic RV: 7pts (17.5\%); $18 \mathrm{RA}$ isomerism (45\%), 3 LA isomerism (7.5\%), 10 TAPVD (25\%), 1 situs inversus and 1 situs ambigus.

III group biventricular repair: $32 \mathrm{pts}(43.8 \%)$ with the following cardiac associations: 6 TOF $(18.7 \%), 6$ CoAo \pm HAA $(18.7 \%), 4$ RVOTO (12.5\%), 5 small RV (15.7\%) and 11 small LV (34.4\%).
IV group 1 ventricle repair: $1 \mathrm{pt}$ (1.4\%) with TOF and small RV. Results: 5 years median follow-up.

I group: 66pts (98.5\%) had complete repair of CAVSD; 3 (4.5\%) underwent reoperation in a median time of 7 months (1-25). Early mortality: $1 \mathrm{pt}(1.5 \%)$ for severe PHT; late mortality: $2 \mathrm{pts}$ $(3 \%), 1$ subsequently to myocarditis and 1 for congenital bronchial abnormalities. 61pts $(96.8 \%)$ have trivial or non MR.

II group: 25 survivors $(64.1 \%), 13(52 \%)$ had completion of Fontan, $12(48 \%)$ C-P connection.

III group: 26 survivors (81.25\%); in pts with TOF early and late mortality were $0 \%$; in CoAo $+/-$ HAA early mortality: $1 \mathrm{pt}$ (16.6\%), late mortality: 2pts (33.3\%); in RVOTO early mortality: $0 \%$, late mortality: $1 \mathrm{pt}(25 \%)$; in pts with small RV early and late mortality were $0 \%$; in pts with small $\mathrm{LV}$ early mortality: $1 \mathrm{pt}(9 \%)$ for sepsis and 1 late sudden death (9\%).

IV group: no complications.

Conclusions: Early and mid term results are excellent for simple CAVSD repair or for complex CAVSD where a biventricular repair can be performed. The results are less satisfactory when it is necessary to use a single ventricle repair. We should improve the definition of the anatomy preoperatively to better define in which group to place the CAVSD with "borderline" LV.

\section{9}

Inhaled nitric oxide (iNO) and GMPc blood levels: proposal for a new therapeutical strategy in pediatric cardiac surgery

E. Mazza, C. Panzeri, R. Rigolini

Istituto Policlinico San Donato, S. Donato Milanese (MI), I

Our clinical experience with the use of iNO starts in 1993 and includes 68 pts undergoing corrective surgery, mean age 4 mo $(2 \mathrm{wk}-9 \mathrm{mo})$ and mean weight $3.3 \mathrm{Kg}(2.7-6.8 \mathrm{Kg})$. The underlying heart defects were: complete av septal defect $50(90 \%$ with Down's syndrome), truncus arteriosus 6, total anomalous pulmonary venous drainage 6 , single ventricle undergoing modified Fontan operation 2 and 4 pts with $L V$ and RV assisted device (LVAD, RVAD) for LV and RV failure. Mean dose of iNO was $18+8$ ppm, mean duration of treatment was $5+4 \mathrm{~d}$, mean oxygen saturation under treatment was $98+2 \%$, mean $\mathrm{FiO} 20.6$. Mean intubation period $8+4 \mathrm{~d}$. There were 3 deaths, 1 for sepsis the other 2 for tefractory hypertensive pulmonary crisis. Among our study group, $10 \mathrm{pts}$, with the longest period of treatment (mean of $8 \mathrm{~d}$ ), developed hypertensive crisis upon discontinuation of treatment with iNO and required a longer period of endotracheal intubation.

Conclusion: iNO is an indispensable therapeutical tool in the post operative management of pediatric cardiac pts but 1) the use of iNO reduces the production of endogenous NO (normally restored after $48 \mathrm{hr}$ from the ECC; 2) during the weaning from NO low saturations should be accepted until the pulmonary endothelium restores its capacity of producing endogenous $\mathrm{NO}$; 3) the longer the period of iNO treatment, the more difficult is to wean the patients with subsequent prolongation of their endotracheal intubation: this may lead to significant adverse effects.

We confirm our conclusion by dosing GMPc levels as a final messenger of the iNO. The GMPc dosage was effected as follows: Before cardiopulmonary bypass; 2) After cardiopulmonary bypass; 3) During iNO therapy; 4) At INO withdrawal 5) But primarily during "Rebound" episodes after iNO withdrawal. The laboratory and clinical data obtained in 6 pts showed: a direct correlation between iNO, GMPc and endogenous $\mathrm{NO}$, a possible negative feed-back during iNO therapy and an increase of GMPc levels during and after rebound episodes, suggesting to continue this new therapeutical approach. 
;0

Total cavopulmonary connection in patients with unctionally single ventricle and regurgitation of ttrioventricular valves

I. Tlaskal, B. Hucin, V. Chaloupecky, O. Reich, R. Gebauer, J. Marek Cardiocentrum, Lniversity Hospital Motol, Prague 5, CZR

ttrioventricular (AV) valve regurgitation represents an important isk factor of early and late death after total cavopulmonary connecion (TCPC). Between 1991 and 2001, 193 consecutive patients pts) with functionally single ventricle underwent definitive palliaion by TCPC. In $22(11.4 \%)$ moderate to severe AV valve regurgiation was present. These patients underwent repair at a median age If $8,2 \mathrm{yrs}(1,0-29 \mathrm{yrs})$. The diagnosis was set by echocardiography nd heart catheterization with angiocardiography. The main diagnois was DILV in $6 \mathrm{pts}$, DORV in 6, AVSD in 2, MA in 2, TGA in 2, JIRV in 1. EBST in 1, PA/IVS in 1 and CTGA in $1 \mathrm{pt}$. Common $\mathrm{AV}$ valve was found in $11(5(\%)$ pts. In $18(78.3 \%)$ pts one or two revious surgeries had been done earlier. The surgical procedure onsisted in TCPC with lateral tunnel in $15(65.2 \%)$ pts, using :xtracardiac Goretex tube in $7(30.4 \%)$ pts and Kawashima method n $1(+.4 \%)$ pt. Valve repair was possible in 18 pts $(81.8 \%)$ and valve eplacement was necessary in 4 pts $(18.2 \%)$. Fenestration was perormed in $12(5+6 \%)$ pts. Plastic repair consisted in complex annuoplasty in $8(36.4 \%)$, suture or patch closure in $6(33.3 \%)$ and simple commisuroplasty in $4(22.2 \%)$ pts. One $(4.6 \%)$ patient died early and me $(4.6 \%)$ died late, after spontaneous closure of fenestration. This xas one of two patients, in whom moderate to severe regurgitation ersisted, though their clinical condition was transiently good. 20 $90.9 \%$ ) pts are alive and doing well (NYHA class I or II) $0.3-7.0 \mathrm{yrs}$ mean $4.21 \mathrm{yrs}$, SD $1.76 \mathrm{yrs}$ ) after surgery. TCPC with AV valve epair or replacement can be performed with reasonably low early ind late mortality and morbidity even in patients with haemolynamically important $A V$ valve regurgitation.

The study was done with support of a grant VZ FNM 000000 ;4203, No. 6026 .

s1

Jutcome of pregnancy in 147 mothers with zongenital heart disease

V. Fesslova', L. Villa, S. Salmona, B. Acaia

Dpt.Cardiology, Istituti Clinici di Perfezionamento, Milano, I

3ackground: Women with congenital heart disease (CHD) may sresent cardiac complications during pregnancy and labor and heir cardiac state may subsequently deteriorate.

Material and methods: The cardiac state during pregnancy, labor and after delivery was retrospectively analysed in 147 women with CHD (mean age 28 yrs, range 21-41) referred to our Centre for etal echocardiography and maternal condition; 7 women were ollowed during two pregnancies. 131 women had acyanotic CHD, operated in 78, 16 women had cyanotic lesions: 13 Fallot's Tetralogy $(\mathrm{TF}), 1$ transposition of great vessels, 1 pulmonary ttresia and ventricular septal defect (PA + VSD), 1 univentricular reart $(\mathrm{UVH})+$ pulmonary stenosis, all were operated. 31 women were at their second pregnancy and 7 at the third pregnancy.

Results: 14 pts $(10 \%)$ were on specific therapy during pregnancy: 11 for arrhythmias pts. with atrial septal defect with pulmonary aypertension/PHt, TF, Ebstein, aortic coarctation (CoA) and 1ypertrophic cardiomyopathy (HCM); 2 pts were on anticoaguants ( 1 with prosthetic aortic valve and another because of previsus myocardial infarction) and 1 woman continued digoxin for tilated cardiomyopathy (DCM).

The course of pregnancy was complicated seriously in 11 cases $(7.4 \%)$ : in 4 pts by serious arrhythmias and in 4 by worsening of the hemodynamic state (in unoperated ASD $+\mathrm{PHt}$, unoperated $\mathrm{CoA}$ and 2 cyanosed pts); one pt. operated for CoA, with residual isthmic aneurysm, serious arrhythmias and hypertension died at 34 wecks of gestation of rupture of aneurysm, after a hypertensive crisis; one pt. with DCM had acute pulmonary edema postpartum and one pt. on anticoagulants had hemorrhage postpartum. The cardiac state was unchanged during pregnancy and labor in the remaining women, with exception of one intrauterine death due to fetal distress. There were 5 recurrencies of $\mathrm{CHD}$, (3.3\%), of concordant type in 2 .

Conclusions: Our data show a regular course of pregnancy in uncomplicated CHD, while pts. with arrhythmias or important morphofunctional lesions have a more serious prognosis

\section{Session 8: General}

62

Congenital cardiovascular malformations in infants of diabetic mothers; a 6 year prospective study

C. Wren, G. Birrell, G. Hawthome

Freeman Hospital, Newcastle upon Tyne, $L K$

Objective: To examine the prevalence at live birth and the spectrum of structural cardiovascular malformations in infants of diabetic mothers.

Methods: Data on the numbers and types of cardiovascular malformations diagnosed in infancy in babies born to diabetic mothers in 1995-2000 were collected prospectively from the northern diabetic pregnancy survey. Similar data on offspring of non-diabetic pregnancies were obtained from the regional paediatric cardiology database.

Results: During the 6 years of the study there were 192618 live births. 609 babies were born to diabetic mothers and $22(3.6 \%)$ of them had cardiac malformations. $1417(0.7 \%)$ babies from nondiabetic mothers had a cardiac malformation. The odds ratio for congenital heart disease in the offspring of diabetic mothers was 5.0 $(95 \%$ CI 3.3-7.8). Combining our findings with the few small published studies and comparing them with the spectrum of defects in non-diabetic pregnancies shows a substantial excess of transposition of the great arteries, truncus arteriosus and tricuspid atresia.

Conclusions: This first population-based prospective study babies of diabetic mothers shows a five-fold excess of cardiovascular malformations in general and an even greater excess of some specific diagnoses.

63

Increased levels of P-BNP and P-ANP after stage I but not stage II operation in children with single ventricle defects

D. Holngren, A. Westerlind, G. Lindstedt, P.-A. Lundberg, H. Whlander Department of Pediatric Cardiology, Göteborg, $S$

Introduction: Children with single ventricle undergo a stepwise procedure towards a Fontan type of circulation. After stage I the functioning ventricle is responsible for both the systemic and pulmonary circulation resulting in a volume overloaded ventricle. After stage II the pulmonary circulation is achieved by passive venous return from the superior caval vein thereby reducing the volume overload. Brain (BNP) and atrial natriuretic peptide (ANP), produced in the ventricles and atria of the heart, has been shown to be increased in plasma during systolic dysfunction and different types of hemodynamic overload of the heart.

Aim: To study if P-BNP and P-ANP reflect differences in ventricle volume load after stage I and II operation, respectively, in children with single ventricle. 
Methods: Consecutive blood samples for analysis of P-BNP and $\mathrm{P}$-ANP during the regular pre-operative investigation, preceding the stage II and III operations. Cardiac function was evaluated by echo-doppler investigation and blood oxygenation by pulse oxymetry. As controls, blood samples was drawn from children at similar ages with normal hearts.

Results: Blood samples were obtained from 13 controls and 6 patients after stage I and 10 after stage II. One child from the stage I and II groups respectively, were excluded from further analysis due to poor ventricular function. Nine children had mild AV-valve regurgitation. Three in each of the groups stage I and II had a morphologic right ventricle as the systemic ventricle. The median values for P-BNP and P-ANP were higher $46.9 \mathrm{ng} / \mathrm{L}$ (range $20.8-90$ ) and $99.4 \mathrm{ng} / \mathrm{L}$ (range 17.1-203) after stage I than in the controls $4.7 \mathrm{ng} / \mathrm{L}$ (range $0-9.5)(\mathrm{p}=0.01)$ and $32.0 \mathrm{ng} / \mathrm{L}$ (range $11.7-+7.3)(\mathrm{p}=0.02)$ and after stage II $8.1 \mathrm{ng} / \mathrm{L}$ (range $0.6-15.3)(\mathrm{p}=0.01)$ and $28.2 \mathrm{ng} / \mathrm{L}$ (range 17-54.4) $(\mathrm{p}=0.02)$. Median $\mathrm{O}_{2}$-saturation was 76\% (range 73-81) after stage I and $84 \%$ (range 80-86) after stage II $(\mathrm{p}=0.01)$. Conclusion: Plasma levels of BNP and ANP were increased after stage I operation in children with single ventricle defects. After stage II operation P-BNP and P-ANP were similar to control children. Thus, P-BNP and P-ANP seem to reflect the volume overload of the systemic ventricle after stage I operation.

\section{4}

Effect of Idebenone treatment on cardiac parameters in Friedreich ataxia patients

L. Mertens, F. Weidemamm, I. Matthy's, B. Eyskens, M. Geurillig,

G. Di Salvo, D. Boshoff, G. Sutherland, L. Hatle, J. Van Hove, G. Buyse University Hospitals Lemen, Lemen, B

Background: Hypertrophic cardiomyopathy in Friedreich ataxia (FA) is thought to be caused by respiratory chain deficiency due to mitochondrial iron accumulation and oxidative stress. Idebenone, a potent free-radical scavenger, has been used in FA patients in two preliminary studies with conflicting results on left ventricular mass. Aim of our study: Investigate the effect of long-term administration of Idebenone on left ventricular mass index (LVMI) $(\mathrm{g} / \mathrm{m} 2)$ and on regional myocardial function, in FA patients with cardiac hypertrophy, using standard echocardiography and, Strain Rate (SR) (unit S_1) and Strain (e) (\%) imaging, a new non invasive method to quantify regional myocardial function.

Methods: Seven FA patients with hypertrophic cardiomyopathy were studied baseline (B) and after 12 months of therapy (AT) with Idebenone $(360 \mathrm{mg} / \mathrm{d})$ using standard echocardiography and SR and e.

Results: After 12 months of therapy we found a significant improvement in ejection fraction $(\%)(B=7710, A T=864, p=0.02)$ and a significant reduction in LVMI $(B=12629, A T=10126$, $p=0.02$ ). Only in 1 patient we did not find reduction of LVMI. After therapy in FA patients there was an improvement of e and SR in all the studicd segments but it rached a significant value only in SR of lateral wall middle segment $(B=-0.80 .2$ vs. $A T=-1.50 .7$, $p=0.03)$ and an almost significant value in the basal and apical segments of the lateral wall ( $p=0.017$ and $p=0.06$, respectively).

Multivariate analysis showed that septum basal segment SR $(p=0.0099$, Coeff. Stand. 1.283), posterior wall SR $(p=0.02$, Coeff. Stand. -0.866$)$ and septum middle segment e $(p=0.0045$, Coeff. Stand. $=1.221)$ were significantly correlated with LVMI. Conclusion: In our sample long-term administration of Idebenone is able to improve significantly LVMI, ejection fraction and regional myocardial function.

We suggest to use SR and e imaging in follow-up of these patients to evaluate regional myocardial function.
65

Coagulation profile and liver function in patients after total cavopulmonary connection

V. Chaloupecky', I. Svobodova, I. Hadacon, V. Tomek, B. Hucin,

T. Tlaskal, J. Marek, J. Skovranek

Kardiocentrum, Lniversity' Hospital Motol, Prague, CZR

Objective: To examine coagulation factors and liver function tests in patients after the total cavopulmonary connection (TCPC) Method and patients: A prospective study evaluating clinical. echocardiographic, hematology and biochemistry data in $105 \mathrm{pts}$ aged 4 to $24 \mathrm{yrs}$ (median $10 \mathrm{yrs}$ ) at 1 to 8 yrs (median $5 \mathrm{yrs}$ ) after surgery. All pts were on low dose of aspirin, another 23 pts on warfarin therapy were excluded from the study. Coagulation factors were compared with age matched normal values.

Results: $96 \%$ of pts were in a good clinical condition (NYHA I or II) and no thrombi were observed on echocardiography. The global coagulation tests were in general within normal limits. Factor VII was abnormally decreased in $21 \%$ pts, factor V in $13 \%$ pts, protein $\mathrm{C}$ in $10 \%$ pts and antithrombin III in $5 \%$. More than half of pts had increased level of plasminogen. Serum aspartate aminotransferase was increased in $30 \%$ pts, total bilirubin in $27 \%$ and gamma-glutamyltransferase in $55 \%$ pts. Total protein, albumin, and prealbumin were normal almost in all pts. There was a significant correlation between prothrombotic factors (INR. factor VII) and antithrombotic factors (protein C, plasminogen. antithrombin III). There was also a significant correlation between prealbumin and coagulation factors produced by the liver.

Conclusion: Decreased synthesis of pro- and anti-thrombotic factors due to hepatic dysfunction was obscrved in some patients after total cavopulmonary connection. The overall coagulation homeostasis seemed to be in balance in our selected group of patients. Supported by grant NA5968-3, Ministry of Health.

\section{6}

Clinical characteristics and effect of new treatments in pediatric primary pulmonary hypertension

A. Manes, N. Crali, D. Prandstraller, A. Donti, R. Formigari,

G. Bronzetti, M. Aquilina, G. Boggian, F. Pelino, A. Branzi, F.M. Pichio

Istituto di Cardiologia, Lnimersit di Bologna, Bologna, Italy, I

Primary pulnonary hypertension (lPH) is a severe disease that involves predominantly individuals in the fourth and fifth decade of life. Nevertheless, PPH is observed also in pediatric age and the clinical characteristics and the outcome of these subjects are not well known. In addition, the new treatments for PPH has been tested predominantly in adult patients and few data are available in the pediatric group.

The purpose of the present study is the clinical and therapeutic comparison of a cohort of $15 \mathrm{PPH}$ patients less or equal than 15 years of age (P-PPH, mean age 8.4 4.6, range $1-15$ years) with 40 adult $\mathrm{PPH}$ patients (A-PPH, mean age 4416 range $17-71$ vears). Female to male ratio was $0.75: 1$ in $\mathrm{P}-\mathrm{PPH}$ and $1, \overline{5}: 1$ in $\mathrm{A}-\mathrm{PPH}$. Family history was present in $7 \%$ of P-PPH and $12.5 \%$ of A-PPH. The following baseline hemodynamic variables of $\mathrm{P}-\mathrm{PPH}$ and A-PPH are shown in the table: heart rate (HR), right atrial pressure (RAP), pulmonary artery pressure (PAP), mean blood pressure $(\mathrm{mBP})$, cardiac index $(\mathrm{CI})$, pulmonary vascular resistance index (PVRI), arterial $\mathrm{O}_{2}$ saturation $\left(\mathrm{SaO}_{2}\right)$.

$40 \%$ of $\mathrm{P}-\mathrm{PPH}$ were considered responders to acute vaso-reactivity tests as compared to $20 \%$ of A-PPH. Chronic prostanoid treatment (intravenous, inhaled or oral) was administered in $66 \%$ of $\mathrm{P}-\mathrm{PPH}$ as compared to $45 \%$ of A-PPH. Survival of P-PPH treated 
with prostanoids $(\mathrm{n}=10)$ was $80 \%$ after 36 months as compared :o $50 \%$ survival in P-PPH not treated with prostanoids $(n=5)$. In conclusion, P-PPH patients seem to have higher PAP and PVRI and lower MBP at baseline cardiac catheterization as comsared to A-PPH. The prevalence of responders to acute vasoeactivity tests is higher in P-PPH as compared to A-PPH. Vevertheless, more P-PPH patients seem to require prostanoids ind the survival seems to be improved by this treatment.

\section{7}

nhaled iloprost: an alternative therapy to continuous prostacyclin infusion for pulmonary arterial hypertension in children

II. Fasnacht, P.C. Rimensberger, R. Burger, M. Beghett Cinversity Children's Hospital Zurich, Ziurich, $\mathrm{CH}$

ntroduction: Pulmonary arterial hypertension (PHT) is a rare lisease in childhood associated with poor prognosis. Long term nfusion of prostacyclin has somewhat improved its prognosis but equires a permanent central venous catheter often related to seriJus side-effects (infections, thrombosis, interruption of therapy issociated with a pump or catheter dysfunction). Inhaled iloprost, I prostacyclin analogue, has shown beneficial effects in adults and nay offer an alternative approach in children as well.

Method: Since 1998,7 patients aged 34-22+ months (median: 100) were treated with inhaled iloprost with a follow up of $6-45$ months median:14). PHT was primary in 4 and secondary in 3 patients. til had right heart catheterization (RHC) with nitric oxide (7/7) Ind iloprost (2/7) testing before treatment. RHC was performed ifter 1 year of treatment in 4 patients. In $4 / 7$ exercise testing (or imin. walk test) before and after 1 year of treatment was perormed. One patient did not have RHC or exercise test after 1 year of treatment. Iloprost was administered by inhalation using comnercial aerosol devices $5-6$ times per day at a dosage of 50 to $100 \mathrm{~g}$ I day:

Results: All patients being treated with inhaled iloprost are still live. There were no significant changes in pulmonary vascular esistance (Rp) 1 year after treatment, but a tendency to a lower $3 \mathrm{p}$ after trearment, of $1-5 \mathrm{U} / \mathrm{m} 2$, was observed in all patients ested. However, in $5 / 7$, a remarkable clinical improvement xccured, with improved exercise tolerance, reduced cvanosis and setter social integration (back to school). Changes of NYHA Jlass 4 to 2 occured in 1 , from 3 to 2 in 2 patients. In $2 / 7$, there xas no clinical improvement. These 2 are awaiting lung transplant. Vo side effects were observed.

Zonclusions: These preliminary datas show, that inhaled iloprost eads to a stabilisation and often to a remarkable clinical improvenent, with improved exercise tolerance with subsequent better ocial integration. There were no deaths and no local or systemic ide effects under treatment. Inhaled iloprost may offer a safe alterrative to continuous prostacyclin infusion in pediatric patients and sbviates the need of a permanent central venous catheter.

\section{8}

Cardiovascular gene polymorphisms in paediatric jatients with systemic or pulmonary hypertension

: Kreuder, A. Dost, I. Wichel-Behnke, R. Zimmerman,

K.J. Hagel, D. Schranz

Department of Paediatric Cardiology, Justus Liebig-Liniversit, Giessen, D

Jenetic variability has been suggested to significantly contribute to he development of hypertension and vasculoproliferative disorders $n$ the systemic and pulmonary circulation in adults. However, the elevance of cardiovascular gene polymorphisms has not been examined in paediatric patients at risk for or with established hypertension or vasculoproliferative disorders.

Patients: This study included 111 patients with diabetes mellitus type 1 (IDDM) (mean age 11.5 yrs, mean HbA1c 7.4\%), 27 patients with late or persistent systemic hypertension after surgical or interventional correction of aortic coarctation (age 8-20yrs, time after correction 5-19yrs), and 24 patients with pulmonary vasculopathy $(\mathrm{Rp} / \mathrm{Rs}>0.7)$ due to primary pulmonary hypertension $(\mathrm{n}=9)$, congenital cardiac malformations $(n=11)$ and bronchopulmonary dysplasia $(n=4)$.

Methods: Genomic polymorphisms of endothelial NO synthase (eNOS), 2 adrenergic receptor, genes of the renin-angiotensinaldosterone system and the 3 subunit of the $G$ proteins were examined. Alleles were differentiated by RFLP analysis, microsequencing (SnaPshot-assay, $\mathrm{ABI}$ ) and allele specific oligonucleotides (SDS 7700, ABI).

Results: In IDIDM patients, the risk alleles of the angiotensinconverting enzyme (ACE, 279 bp deletion, intron 16) and of angiotensinogen (AGT, $-704 \mathrm{~T}$ ) were found to be significantly associated with the systolic $24 \mathrm{~h}$-blood pressure profile (day, night, day-to-night-variability) $(\mathrm{p}<0.05)$. These alleles were previously found to be associated with higher levels of ACE and AGT. In contrast, blood pressure profiles were independent of $\mathrm{HbA} 1 \mathrm{c}$ values, body mass index and IDDM duration. Patients with pulmonary hypertension showed an increased frequency of the $79 \mathrm{C}=27 \mathrm{Q}$ allele of the 2 -adrenoreceptor (diminished vasodilation in vivo, $\mathrm{p}<0.05$ ) and of the $-786 \mathrm{C}$ variant of eNOS (diminished NO synthesis, $\mathrm{p}=0.072$ ). In systemic hypertension after correction of aortic coarctation, a statistical trend was found for an association with the AGT $-704 \mathrm{~T}$ allele $(\mathrm{p}=0.07)$ and the intron 4-27 bp deletion variant of eNOS $(p=0.079)$.

Conclusions: Also in paediatric patients, associations between distinct cardiovascular gene polymorphisms and hypertension in systemic and pulmonary circulation may be detectable. If proved in larger patient groups, these genetic variants may be important for improved risk stratification and more individualized therapeutic approaches.

\section{9}

\section{Ryanodine receptor gene ( $\mathrm{Ry} R 2)$ mutations in the paediatric age}

B. Bauce, A. Rampazzo, C. Basso, A. Bagattin, L. Daliento,

G. Thiene, G.A. Danieli, A. Nava

Department of Cardiology, Lniversity of Padia, Padova, I

Background: RyR2 mutations have been demonstrated to be linked to effort-induced polymorphic ventricular arrhythmias (PVA) and juvenile sudden death (SD), either in patients with normal heart or with a mild form of arrhythmogenic right ventricular cardiomyopathy (ARVD2). The aim of the study was to define the clinical spectrum of the disease and the efficacy of the ongoing therapeutic strategies in a paediatric population of subjects carrying RyR2 mutations.

Methods and results: 17 subjects with RyR 2 mutations who were younger than 18 years at the time of the first visit comprised the study group. They were $8 \mathrm{M}$ and $9 \mathrm{~F}$, age ranging 6-18 yrs, mean $12 \pm 3.8$. Two subjects were examined due to ventricular arrhythmias (cardiac arrest and PVA respectively), one due to a syncopal episode while the remaining 14 cases were diagnosed as having $\mathrm{RyR} 2$ mutations during familial screening. Ten subjects showed PVA during stress test $(6 \mathrm{M}, 4 \mathrm{~F}$, mean age $13.7 \pm 2.6 \mathrm{yrs})$. Of them, in 5 PVA were present at the first visit (mean age $15.6 \pm 2.4 \mathrm{yrs}$ ), while in the remaining they appeared during follow-up (mean age $17 \pm 2.9 \mathrm{yrs}$ ). Five subjects had syncopal 
episodes before the diagnosis (mean age $14.4 \pm 2.5 \mathrm{yrs}$ ). PVA and/or syncope were never detected in subjects younger than 12 yrs. One subject died suddenly at the age of 20 , without antiarrhythmic treatment. Six subjects are asymptomatic so far (2 M, 4F mean age $8.6 \pm 2.9 \mathrm{yrs}$ ). All young patients with PVA underwent beta-blocker therapy; during follow-up (range 1-15yrs, mean 6.7), none of them experienced syncope or died suddenly.

Conclusions: The lack of PVA and syncope in subjects aged $<12$ yrs carrying RyR 2 mutations supports the existence of a concealed phase during childhood. Follow-up data prove the efficacy of beta-blocker therapy in preventing SD. Since the risk stratification of SD in asymptomatic carriers is a challenge, a preventive strategy consisting of beta-blockers therapy in all subjects $>12$ yrs and repeated stress tests in younger carriers is recommended.

\section{0}

Lung volumes and pulmonary gas exchange at rest and during exercise in patients with Fontan circulation at a long-term follow-up

E. Stömvall-Larsson, B. O. Eriksson, R. Sixt

Dept. of Pediatric Cardiology', Göteborg, $S$

The hemodynamic situation for patients with Fontan circulation is characterised by a reduced pulmonary blood flow. To evaluate if this has any implication on the lung function, we studied the surviving patients operated upon at our institution between 1980-1991. Static and dynamic spirometry, as well as pulmonary gas exchange at rest and during exercise was measured. There were 10 females and 10 males with a mean age of 23.3 years $(11.6-54.4)$ and a mean follow-up time of 13.0 years $(7.8-20.3)$. All patients performed the spirometric studies, while $15 / 20$ fulfilled the exercise tests.

Methods: Lung volumes and flow-volume curves were obtained using a pneumotachograph-based computer system. The diffusion capacity for carbon monoxide (DCLO) was assesed using the single-breath technique. The ventilatory distribution was analysed with the single-breath nitrogen wash-out method (N2-slope). The exercise tests were performed on an electrically braked bicycle ergometer using a steady state protocol with increments in load every 6th minute. Continuous analyses of expired air for determination of ventilation (VE), oxygen uptake (VO2), respiratory rate (RR), carbon dioxide production (VCO2) and heart rate (HR) every twenty second were done. Arterial blood gases were achieved at the end of each work load.

Results: The lung volumes (VC, TLC, FEV1, MEF25 and MEF50) were significantly lower than expected $(p<0.05)$, as well as DLCO and N2-slope $(\mathrm{p}<0.001)$. The alveolar ventilation (VA) increased in a normal way from $8.11 / \mathrm{min}$ at rest to 50.1 at maximal exercise and the VA/VO2 ratio from 29.1 to 34.6 . The alveolar oxygen partial pressure (PAO2) was at rest $14.9 \mathrm{kPa}$ and increased to 16.0 at maximal exercise, while the alveolar-arterial oxygen partial pressure difference (PA-aO2) rose from 5.2 to $8.0 \mathrm{kPa}$. The dead space/tidal volume ratio $(\mathrm{VD} / \mathrm{VT})$ was at rest 0.29 and at maximal exercise 0.26 .

Conclusion: Fontan patients have small lung dimensions, probably due to reduced pulmonary blood flow. This does not influence on their $\mathrm{VD} / \mathrm{VT}$ ratio, VA, VA/VO2 ratio or $\mathrm{PAO} 2$. The $\mathrm{PA}-\mathrm{aO} 2$ is decreased at rest and decreases even more during exercise, partially due to intrapulmonary and/or intracardiac right-to-left shunting.

\section{Session 9: Cathlab - Intervention}

71

Feasibility and efficacy of $R$ wave synchronized atrial pacing in postoperative junctional ectopic tachycardia using a modified commercially available temporary pacemaker

J. Janowsek, P. Vojtovis, R.A. Gebauer

Kardiocentrum, Lniv. Hosp. Motol, Prague, CZR

Atrioventricular (AV) dyssynchrony plays a major role in the development of severe hemodynamic compromise during postoperative junctional ectopic tachycardia (JET). R wave synchronized atrial pacing has a potential for achieving optimal AV synchrony at intrinsic heart rate and spontaneous ventricular activation sequence, but has not been used clinically because of lack of appropriate commercially available equipment.

Methods: The utility of a modified commercially available temporary dual-chamber pacemaker (PACE $203 \mathrm{H}$, software version $1.10 \mathrm{jj}$, Dr. Osypka GmbH, Berlin, Gcrmany, minimum programmable PVARP $=100 \mathrm{~ms}$ ) for $\mathrm{R}$ wave synchronized atrial pacing was evaluated in 10 consecutive pts with postoperative JET (median maximum heart rate 185 , range $130-240 \mathrm{bpm}, 1: 1$ retrograde conduction in $6 / 10$, AV block during atrial overdrive pacing in 3/10) aged $0.3-45$ (median 5.2) months. The pacemaker was programmed to the VAT mode associated with inverse connection of the pacing wires (atrial wires to ventricular channel, ventricular wires to atrial channel, effectively AVT mode). PVARP was set to $100 \mathrm{~ms}$ to enable $\mathrm{R}$ wave sensing shortly after the ventricular (effectively atrial) pacing pulse. AV (effectively ventriculoatrial, VA) delay was initially programmed to tachycardia CL-100 ms to activate atria just before the next spontaneous junctional beat and was finally fine-tuned to achieve maximum increase in arterial pressure. Results: Pacing was successfully applied in all pts over a median period of 29 (range 10-96) hours until tachycardia cessation and led to an immediate increase in systolic, mean and pulse pressure by $8.93 .2(\mathrm{p}<0.001), 8.14 .0(\mathrm{p}<0.001)$ and $11.97 .8 \%$ $(\mathrm{p}<0.005)$, resp. Pacemaker mediated tachycardia developed in 2 pts and was stopped by AV (effectively VA) delay prolongation. Improved hemodynamics allowed for a decrease in inotropic support in all pts with presumed suppressive effect on tachycardia rate. Conclusion: $\mathrm{R}$ wave synchronized atrial pacing, performed with a modified conmercially available temporary dual-chamber pacemaker, carried significant hemodynamic benefit and should be included into the standard management protocol of postoperative JET.

\section{2}

A comparison of conventional mapping versus non-contact mapping of intraatrial reentrant tachycardias in patients after atrial switch procedures for d-transposition of the great arteries

T. Kriebel, B. Franzbach, B. Windhagen-Mahnert, H. Kditz,

H. Bertram, J. Tebbenjohanns*, T. Paul

Department of Pediatric Cardiology, Hannover, $D$

13 patients with d-transposition of the great arteries after Mustard/Senning procedure underwent electrophysiological study for recurrent intraatrial reentrant tachycardia (IARTs). In 8 patients, a 20 -pole electrode catheter and a steerable $7 \mathrm{~F}$ mapping and ablation catheter were placed in the systemic venous atrium, and via the retrograde route in the pulmonary venous atrium, if required. During IART pacemapping and entrainment mapping were performed in order to localize protected areas of atrial tissue 
etween anatomical and/or surgical barriers of electrical isolation. a 5 patients the systemic venous atrium was studied with the on-contact mapping system (Ensite $3000 \mathrm{Tm}$ ).

a patients studied with the Ensite $3000 \mathrm{TM}$ system detailed propgation of the atrial activation front could be assessed including 1e reconstruction of a complete loop of the reentry circuit in 2 atients. Linear radiofrequency current lesions were induced in rder to eliminate conduction through the identified areas of atrial ssue. IART was not inducible in 12 of the 13 patients during speated stimulation ( 7 of 8 patients using conventional mapping nd in 5 of 5 patients using the non-contact mapping technique). 4ean duration of the electrophysiological study was 202 minutes. Aean fluoroscopy time was 21.6 minutes. There were no statistially significant differences in fluoroscopy time $(p=0.46)$ and rocedure time ( $\mathrm{p}=0.12$ ) using the Ensite $3000 \mathrm{TM}$ system comared to conventional mapping. After a mean follow-up of 20 conths 10 of 12 patients with initially successful procedures are -ee of tachycardia.

The use of the modern non-contact mapping system introduced ecently into clinical practice allowed precise assessment of atrial ctivation sequence, the prerequisite for effective radiofrequency urrent application.

3

ixperimental evaluation of a new angled implatzer duct occluder

L. Bass, H. Kong, X. Gu, M. Lrness, J. Titus, D. W. Hunter

iniversity of Minnesota, Mirneapolis, $M N, L S A$

I new Amplatzer device developed for closure of patent ductus rteries (PI)A) was designed. Mesh density of 144 thin 0.004 inch) Nitinol wires allowed elimination of fabric patches. the aortic retention disc is angled at 32 to the cylindrical portion nd concave maximizing approximation with the aorta when leployed. The downstream end of the aortic disc has a platinum narker. Seven dogs had a $6 \mathrm{~mm}$ diameter, $10 \mathrm{~mm}$ length Gore-Tex PDA" surgically created at an angle approximating normal PDA rientation. Right-sided cardiac catheterization was performed inder general anesthesia. Aortography was performed to define he PDA. A device frame was chosen 1-2 mm larger than he PDA; loaded and advanced through a $6 \mathrm{~F}$ venous guiding atheter

Cook) across the PDA. The retention disc was deployed in the lescending aorta, rotated clockwise until the rim maker was riented downstream if necessary, and the cylindrical portion leployed in the PDA. Aortography confirmed good position and he device was released by unscrewing counter-clockwise. tortography was repeated in 30 minutes. Device orientation was maged by cine in profile and en face. Follow-up cardiac catheterzation is planned 1 week, 1 month, and 3 months after device lacement. Sacrifice will be performed at 3 months with pathoogical examination. Complete occlusion occurred in all animals y 30 minutes after placement with no obstruction of the aorta or ulmonary artery. Device orientation varied between 5 and 7 'clock. The aortic retention disc conformed closely to the aorta. The new angled Amplatzer PDA device retains the advantage of woiding aortic obstruction, but is significantly easier to deploy. Jensity of the wire mesh obtained by increasing the wire count to 144 allows elimination of fabric, and deployment through a maller guiding catheter. Rotation into proper orientation is easly performed without a special orienting delivery system. Despite he absence of fabric baffles, complete occlusion occurred in $100 \%$ of experimental animals.
74

European multicentre study on transcatheter ASD closure with the Helex septal occluder

F. Maymone-Martins, H. Sievert, J. Hess, P. Etwert, M. Carminati, M. Sclmeider, A. Salmon, S. Qureshi, J. De Giovanni, N. Wilson

Hospital de Santa Cruz, Carnaxide, $P$

Background: Previous experience suggests that the Helex septal occluder is a good alternative for the transcatheter closure of Ostium Secundum ASDs. This device is a non self-centering double disk of ultrathin ePTFE mounted on a wire frame of nitinol, inserted via a special 9F catheter. After deployment the device assumes the form of a flat, circular, soft and flexible prosthesis. A device/defect ratio of $1.8 / 1$ to $2 / 1$ is recommended. Delivery is technically demanding but the device can be repositioned and retrieved at any time prior to release.

The purpose of this study was to further substantiate the safety and efficacy of this device in a Multicentre Europcan study.

Methods: The study included all patients (pts) in whom implantation was attempted. Pts were catheterised under general anaesthesia and transesophageal echo guidance. They were reviewed 1,6 and 12 months $(M)$ later with ECG, chest X-Ravs and transthoracic echo.

Results: Device implantation was attempted in 110 pts and achieved in $108(98 \%)$. Follow-up data includes $105 \mathrm{pts}$. Their ages ranged from 1 to 75 (mean 22) years and weights from 8 to 102 (mean 43 ) Kg; 68\% were female. Balloon stretched diameters ranged from 5 to 21 (mean 13) $\mathrm{mm} ; 18$ pts had multiple defects and 8 had septal aneurysms. Follow-up times ranged from 9 to 866 (mean 353 ) days. Complete closure was noticed in $66 \%$ of the pts prior to discharge, in $82 \%$ at $6 \mathrm{M}(\mathrm{n}=4 \overline{5})$ and in $90 \%$ at $12 \mathrm{M}(\mathrm{n}=29)$ the remaining having trivial/small shunts. In 5 pts embolization occurred during the procedure or within $24 \mathrm{hrs}$. Percutaneous retrieval was achieved in all, followed by the insertion of a larger Helex in 2 and Amplatzer in 3. The latter are not included in the follow-up. Late wire fracture occurred in one instance.

Conclusions: The Helex septal occluder is safe and efficient for the percutaneous closure of Ostium Secundum ASDs. Its advantages include a low profile with little metal and a flexible ePTFE patch. Its non self-centring mechanism makes it especially convenient for multiple ASDs. There is a low incidence of residual shunts that decreases with time.

75

Interventional closure of patent foramen ovale and atrial septal defects after paradoxical embolic events A. Beitzke, H. Schuchlenz, M. Beitzke, A. Gamillscheg,

J.I. Stein, P. Zartner

Pediatrische Kardiologie, Univi-Kinderklinik, Graz, A

251 patients with a persistent foramen ovale (PFO), mean age $43,312,4$ vears and 17 patients with an atrial septal defect (ASD), mean age 3810,5 years underwent catheter closure between $6 / 1995$ and 6/2001. 149 had an ischemic stroke, 108 a transient ischemic attack or prolonged reversible ischemic neurologic deficit, 5 peripheral arterial embolism, 4 suffered from decompression sickness after diving and 2 had transient global amnesia. 62 of them had multiple events in spite of antiplatelet or anticoagulant therapy. The patients received 5 different devices: 13 Rashkind occluders, 32 Amplatzer septal occluders, 109 Amplatzer PFO-occluders, 78 Cardioseal and 36 Starflex devices. Time of fluoroscopy was $8,34,5$ minutes. In three patients a Cardioseal device embolized and had to be removed from the groin vessels. We saw five inguinal or retroperitoneal venous hematomas with 
the need for operation in one. One early and a late perforation of the left atrium caused by a guide wire and a left-atrial disc of an Amplatzer occluder respectively, also needed urgent surgery. 14 patients had documented late arrhythmias. Seven patients with atrial fibrillation or flutter needed drugs or cardioversion while the other patients with runs of supraventricular tachycardia, atrial flutter and multiple extrasystoles needed no therapy. On TEE 6 months after implantation we found six significant residual leaks. All of these leaks occured with larger CardioSeal or Starflex devices. Four of these patients had the defect closed with a second device.

202 PFO-patients and 12 ASD patients were followed for 6 to 62 $(24,614,2)$ months; two died due to a traffic accident and a myocardial infarction respectively. Four patients had another neurologic event following PFO-closure within the first six months. We now overlook 210 patients with 348,6 symptom-free patient years and have a $1,9 \%$ one-year recurrence rate of neurologic events.

Catheter closure of the PFO and atrial septal defect is a simple, effective and quick method which ensures a high closure rate, avoids life-long anticoagulation and has a low recurrence rate of neurologic events. Amplatzer occluders are our preferred occluders with a low incidence of major complications and residual leaks.

\section{6}

\section{Complications of transcatheter closure of ventricular septal defects - results of an international questionaire J. V. De Giovann}

Heart Unit, Birminghan Children's Hospital, Birmingham, LK

Transcatheter closure of ventricular septal defect (VSD) is being performed with increasing frequency partly as a result of specifically designed devices for different clinical circumstances. However, individual operator experience remains small and appreciation of problems or complications cannot be seen in perspective. A questionnaire was sent to known interventionists worldwide asking about their experience, complications encountered and for any recommendations. Twenty eight replied.

Several types of devices were used for different clinical indications/situations. This, together with the different operators and lack of the total number of cases considered or performed creates a limitation in this study. Nevertheless, the following complications were reported:

Tricuspid Valve Damage $=5$, Death $=4$, Mitral Valve Damage $=$ 2, Device Embolisation $=2$, Aortic Valve Damage $=1$, Air $/ \mathrm{Clot}$ Embolus $=1$, Perforation $=1$, Tamponade $=1$, Complete heart Block $=1$, SVC Avulsion $=1$, Aortic Dissection = 1, Bacterial Endocarditis $=1$.

These are some of the problems/complications encountered during transcatheter closure of VSDs. These are an underestimate partly because not all the centres replied and most produced details from memory rather than detailed audit. Moreover, it is not based on intention to treat.

These results give an idea of potential problems that may arise in the course of transcatheter closure of VSD. The real incidence is unclear as the denominator (number of cases performed) is missing in most replies.

Conclusion: This highlights the urgent need for detailed documentation of problems with all the devices available, perhaps through a Register. Some respondents to the questionnaire made suggestions regarding the hardware and the technique and these will be presented.
77

Outcome of balloon valvuloplasty of congenital aortic stenosis: up to fourteen years follow-up O. Reich, P. Tax, J. Marek, J. Skovmek, J. Gilk, H. Bartkow, V. Chaloupeck

Kardiocentrum, University Hospital Motol, Prague, CZR

The retrospective study was carried out to assess results of valvuloplasty (VPL) of congenital aortic stenosis (AS). All the 247 patients treated with the VPL in our institution were included in the study:

The VPL was performed at the age of 0 to 23 years (median 226 days). Body weight at the procedure ranged from 1.5 to $79 \mathrm{~kg}$ (median $7.5 \mathrm{~kg}$ ). Balloon-to-annulus diameter ratio was (mean SD) 0.980 .08 . Fluoroscopy time was 19.411 .3 minutes. Median follow-up period was 3.8 years and ranged up to 14.0 years.

AS gradients (ECHO, $\mathrm{mm} \mathrm{Hg}$ ) were reduced significantly: peak from 8126 to $459(p<0.001)$ and mean from 4915 to 2413 $(\mathrm{p}<0.001)$. Median ECHO-grade of aortic regurgitation increased from 0 to $1(p<0.001)$.

Total mortality was $10.9 \%$ (early $4.0 \%$, late $6.9 \%$ ). A re-intervention was necessary in $63(25.5 \%)$ patients: re-VPL in 17 , surgery in 33 . and re-VPL with a subsequent surgery in 13 . Seven patients required repeated re-VPL, 3 of them with a subsequent surgery: The cause of initial re-intervention was re-stenosis in $40(16.2 \%)$ re-stenosis + insufficiency in $5(2.0 \%)$. insufficiency in $16(6.5 \%)$ and left ventricular hypoplasia in 2 (0.8\%) patients. At 14 years after the procedure, actuarial probability of survival was $882 \%$ and that of surgery-free survival $516 \%$

Risk factors for the VPL failure (death or need for surgery) were tested by multiple logistic regression. Two significant factors were detected (odds ratio, 95\% bounds): bicuspid aortic valve (7.47 $1.22-45.81), P=0.03$ and left ventricular shortening fraction before VPL $(0.001,0.000-0.004) \mathrm{P}=0.001$. The probability of survival/surgery-free survival differed according to the age at VPL: newborns $686 \% / 428 \%$, infants $943 \% / 6110 \%$, and older patients $991 \% / 5512 \%$

Conclusion: VPL is safe and effective initial treatment of congenital AS, however half of the patients require surgical treatment later in the course. Risk factors for VPL failure are bicuspid aortic valve, poor left ventricular function, and low age.

Acknowledgement: Supported by grant NA5963-3, Ministry of Health, CZ

\section{8}

Transcatheter closure of perimembranous ventricular septal defects with an eccentric Amplatzer device: initial human experience

J.L.Bass, R. Arora, W.R. Torres, H. Sievert, J. Masura, B.D. Thanopoulos University of Minnesota, Minneapolis, MN; USA

Transcatheter closure of a perimembranous ventricular septal defect (VSD) is more difficult than for other intracardiac defects because of proximity to aortic and tricuspid valves. Results with a redesigned eccentric Amplatzer device in animals led to attempted closure of perimembranous VSDs in humans.

Modifications include a thin profile to avoid the tricuspid valve. eccentric design to avoid contact with the aortic valve, and a curved delivery system forcing correct orientation of the device. Patients had clinical evidence of a significant left to right shunt through an isolated perimembranous VSD with $3 \mathrm{~mm}$ or more separation from the aortic valve. The largest diameter determined from color flow Doppler was chosen and a device selected at least . 1-2 $\mathrm{mm}$ larger. 
'atients were heparinized. Through-and-through guidewire ccess was achieved. The delivery sheath was advanced into left entricular (LV) apex. The device was advanced to tip of sheath. chocardiography confirmed the sheath tip free of the mitral pparatus. The LV disc was deployed, withdrawn to the septum nder echo, and the other disc deployed. With no aortic insuffiiency, device position was confirmed by echo, fluoroscopy and sft ventriculography, and the device detached.

ixteen patients in five countries participated. Ages ranged from om 1.25 to 32 vears (mean 13.8 years) and weight 10 to $80 \mathrm{~kg}$ nean $+2.6 \mathrm{~kg}$ ). The average Qp/Qs was $1.6, \mathrm{VSl}$ size $6.9 \mathrm{~mm}$ ange 1.6 to $8 \mathrm{~mm})$, and device size $8 \mathrm{~mm}$ (1-4 mm larger, range to $12 \mathrm{~mm}$ ). Device orientation was between $3: 00$ and 7:30 ) clock in twelve. The other four were deployed from the aorta $r$ with a nonstandard delivery sheath. One device was removed or aortic insufficiency that resolved immediately. Fifteen were dccessful with complete closure on angiography and echocadioraphy: There was no aortic or tricuspid insufficiency, no signifiant arrhythmias, no embolizations.

There are few patients. Most were relatively older, large and did ot have extremely large defects or shunts. Long-term follow-up ; not available.

The eccentric device with curved delivery catheter is effective. Complete and immediate closure of a perimembranous VSD can ie accomplished safely and without complication. Further human xperience is warranted.

\section{9}

itent implantation in children with congenital heart

lisease: acute and non-acute complications

I.B.E. Silneider, A. Will, P.E. Lange

Jept. of Ped. Cardiol. Humboldt University Berlin, Charité, Berlin, D

3ackground: Stent implantation has become a widely accepted nethod for the treatment of vessel stenoses in children with conrenital heart defects (CHD). However, the method of stent mplantation is accompanied by various potential complications.

The purpose of this single centre study is to demonstrate various cute and non-acute complications during and after stent implanation in patients with CHD.

Methods: 153 balloon-expandable stents were implanted in 117 essels of 105 patients at the department of pediatric cardiology Zharit Berlin. The stents were implanted into pulmonary artery tenoses $(n=62)$, coarcatations $(n=27)$, ductus arteriosus $(n=42)$, ystemic vein- $(n=13)$ and pulmonary vein stenoses $(n=9)$.

2esults: The only acute complication noted was embolisation of he stent during implantation $(5 / 153) .1 / 5$ was dislocated due to upture of the balloon used for implantation, $2 / 5$ dislocated durng an emergency implantation into the ductus arteriosus and two :mbolized due to a balloon movement.

ate complications were late stent dislocations of $2 / 153$ because of nadequate stent-position, intimal hyperplasia resulting in in-stent itenoses or occlusions in 15 , respectively 9 stents and damage of the irterial vessel at the puncture side in 6 patients. One patient develsped a small aneurysm close to the stent 3 months after implantajon into a narrowed conduit. In another patient with calcified romograft stenosis one of three implanted stents were noted to be sroken in its longitudinal direction without clinical consequences. jurgical treatment including stent removal was necessary in only wo patients. In-stent stenoses due to intimal hyperplasia were reated by re-dilation. However, 9 stents were noted to be comletely occluded by intimal hyperplasia. One patient died of muliple pulmonary vein stenoses 4 months after stent implantations due to severe intimal hyperplasia and vessel occlusions.
Conclusion: Stent implantation in pediatric patients with CHD carries a considerable risk for early and late complications. Some of those complications might be reduced by the change of interventional techniques. However, some late complications like the development of aneurysm formations or intimal hyperplasia are not akrays predictable. Further investigations, particularly on the long term follow up of stents in pediatric patients are needed for the understanding of the mechanism of such complications.

\section{Session 10: Intervention}

80

Perforation of the atretic pulmonary valve: 10 years

follow-up

G. Agnoletti, T.A. Massih, Y. Bondjemline, P. Bonhoeffer,

D. Bonnet, D. Sidi

Necker Enfants Malades, Paris Cedex 15, F

Interventional perforation of the pulmonary valve is considered the elective first stage treatment for pulmonary atresia with intact septum (PAIS). This procedure is strongly advised when the right ventricle is tripartite and the coronary circulation is not right ventricle (RV) dependent. Since $1 / 1 / 90$ to $30 / 12 / 01,38$ newborns with PAIS underwent attempted perforation of the pulmonary valve (17 guide-wire, 21 radiofrequency). $R V$ was tripartite in 26 and bipartite in 12. Mean tricuspid diameter was $10.6+2.3$, range $6-1+\mathrm{mm}$. Perforation was successful in $32(84 \%)$ patients. Right ventricular pressure fell from $112+24$ to $52+15 \mathrm{mmHg}$. There were $6(16 \%)$ procedural complications (2 deaths, 1 perforation. 3 atrial flutter) and $3(8 \%)$ peri-procedural complications (3 necrotizing enterocolitis). Prostaglandin infusion (PGE) was continued for a mean period of $12+8$ (range 1-30) days. At mean follow-up of $12.9+7$ days $20 / 36$ survivors, all under $P G E$, needed cardiac surgery $(10 \mathrm{~B} / \mathrm{T}$ shunts, $10 \mathrm{~B} / \mathrm{T}$ shunt + right outflow patch). There were + post surgical deaths $(20 \%)$. Three more patients had right outflow patch after $9,13,13$ months and t had a re-dilation of the pulmonary valve. At a mean follow-up of $73+43$, range $1-122$ months, $11 / 38$ (29\%) patients (10 with tripartite right ventricle), did not need any surgery, 6 had a bidirectional cavopulmonary anastomosis, none had Fontan correction. All patients are in NYHA class I, 8 have mild to moderate cyanosis. Our results show that perforation of the pulmonary valve: 1 ) is effective in selected patients with normal sized RV, 2) does not avoid surgery in the majority of patients, 3) has a relevant morbidity: An accurate pre-selection of patients is mandatory.

81

Impact of radio-frequency perforation of the pulmonary valve for pulmonary atresia, intact ventricular septum in the neonate: intermediate-term follow-up and outcome R. Johnson, E.J. Ladisans, K.P. Walsh, M. Pozzi, R. Annold Royal Liverpool Children's Hospital, Liverpool, UK

From November 1993, out of 29 consecutive neonates presenting to our institution with pulmonary atresia and intact ventricular septum (PAIVS), 14 with a patent infundibulum and no contraindication to right ventricular (RV) decompression were treated with radio-frequency (RF) perforation of the pulmonary valve. The mean weight was 3.14 (range, 2.()-4.2) kg. There were 6 males and 8 females. The mean angiographic tricuspid valve diameter was 12.30 .1 .1 (1 SD) mm. Tricuspid z-scores ranged from -0.8 to -2.6 . In all cases the perforated outflow tracts were dilated progressively to a mean maximum diameter of $8(7-9) \mathrm{mm}$. The RV pressure fell from mean $97.9(73-130)$ before dilatation to 
$46(31-66) \mathrm{mmHg}$ after RF balloon dilatation. RV: Systemic pressure ratio fell from $1.3(1-1.8)$ to $0.6(0.38-0.71)$ after dilatation $(\mathrm{P}<0.001)$. One patient underwent Blalock-Taussig shunt before RF perforation, because of concern as to size and stenosis of the tricuspid valve. Three other patients required Blalock shunts early after perforation - In one patient because of extracardiac perforation of the infundibulum and cardiac tamponade. The outflow tract was successfully perforated by repeat RF procedure 2 weeks later. The other patients had a shunt 21 and 23 days after catheterisation because of severe persisting cyanosis. Two further patients with persisting cyanosis despite unobstructed RV outflow, underwent trans-catheter stenting of the arterial duct 18 and 21 days after the initial procedure. There were no deaths however 2 patients developed necrotising enterocolitis and one IVC thrombosis and transient renal failure peri-operatively. On follow-up, mean 4 $(0.25-8.2)$ years, 12 patients are well and asymptomatic with unobstructed RV outflow tracts. One patient has had coil occlusion of a stented arterial duct + years later. One patient has undergone superior cavo-pulmonary anastomosis at 2 years and is awaiting completion of the Fontan circulation. One patient died from unrelated meningitis 6 months after the RF perforation. In conclusion, in our experience, primary RF perforation of the RV outflow tract is the treatment of choice in patients with PAIVS, a patent infundibulum, no coronary-dependent sinusoidal communications and a tricuspid z-score $>-2.5$.

\section{2}

Cellular and molecular studies of inflammatory and proliferative response to biodegradable stents produced from iron-based alloys

C. von Schnakenburg, P. Wuebbolt, A. Poppe, C. Fink, G. Hausdorf, M. Peuster

Dept. of Pediatric Cardiology and Pediatric Intensive Care, Göettingen, $D$

Introduction: In vivo studies after implantation of corrosive degradable stents into porcine coronary arteries and the descending aorta of rabbits have demonstrated minimal inflammatory response and neointimal proliferation. The following study was designed to assess whether differences in gene expression of vascular endothelial derived growth factor (VEGF), transforming growth factor beta (TGF-b) and tumor necrosis factor alpha (TNFa) could be demonstrated in vitro.

Methods: Fibroblasts (FB), vascular smoorh muscle (vSMC) and endothelial cells (EC) were isolated from porcine and rabbit aorta and skin. Defined stent segments of a biostable 316-L (PUVA, Devon Medical, Germany) and a corrosive biodegradable ironstent (NOR-I, Devon Medical, Germany) were incubated in cell cultures for up to 7 days. Cultures without stents served as controls. For mRNA-isolation, cells were harvested after $0 \mathrm{~h}, 2 \mathrm{~h}, 1$ day, 3 days and 7 seven days. Proliferation was assessed by cell counts before trypsination. Gene expression studies were performed after reverse transcription using quantitative real-time PCR (Taqman TM, Applied Biosystems, Germany). PCR cycles to reach the threshold of detection (CT) were determined and normalized to the housekeeping gene GAPDH (delta CT method).

Results: EC and FB were adherent, confluent and increased their cellular density during cultivation with $316-\mathrm{L}$ and degradable iron-stents. In contrast vSMC showed a decreased adherence after 3 days, no vital cells were observed after 7 days in cultures incubated with degradable Fe- stents. Incubation with 316-L and control vSMC showed no growth abnormalities. Gene expression encountered non-significant differences of less than 1 PCR cycle in all cell lines and incubation protocols.
Conclusion: Porcine and rabbit EC, FB and $\mathrm{VSMC}$ show no different molecular response to incubation with $316-\mathrm{L}$ and ironstents. Whether cell death of vSMC may potentially reduce neointimal proliferation requires further investigation.

\section{3}

Outcome of modified technique of stent fenestration of the atrial septum

O. Stumper, .M. Getrillig, B. Nagel, J. Vettukattil, .M. Chessa,

J.G.C. Wright

Children's Hospital, Birmingham, UK

Objectives: To develop a modified technique for stenting of the atrial septum in the treatment of patients with a failing Fontan operation or with pulmonary hypertension.

Setting: Tertiary referral centre.

Study design: Prospective ex-vivo experimental and collaborative clinical study

Methods: A stent was mounted on a standard valvuloplasty balloon catheter which was constricted to a predefined diameter by a loop created from a temporary pacing wire. Full balloon inflation created a diabolo shaped stent configuration.

Patients: The above technique was employed in nine consecutive patients (age 4.0-48.2; mean 13.0 (13.8)years) to relief symptoms of a failing Fontan circulation ( $\mathrm{n}=6$ ) or severe pulmonary hypertension $(\mathrm{n}=3)$.

Results: Ex-vivo studies confirmed that a diabolo shaped configuration of a stent could be achieved using the above technique Transcatheter stent implantation was successful in all nine patients All six Fontan patients had significant clinical improvement (Bronchial casts in 1 and protein loosing enteropathy in 5 ). Right atrial pressure decreased from mean 16.8 (2.5) to $13.7(1.9) \mathrm{mmHg}$ $[p<0.05]$, arterial oxygen saturations decreased from $92.8(1.8)$ to $82.7(3.8) \%[\mathrm{p}<0.01]$. One patient died 3 months post stent fenestration from a respiratory illness. Three patients underwent successful stent fenestration in the treatment of pulmonary hypertension All stents are patent and stable at a mean 17.4 (14.1) months followup. No early or late complications were encountered.

Conclusion: This new technique allows placement of a diabolo shaped stent with a predefined diameter across the atrial septum. This increases stent stability, facilitates re-crossing of the stent during future catheter interventions, and ensures medium-term stent patency.

\section{4}

Biodegradable septal occluder: early experimental results H. Bertram, C. Jux, M. Peuster, P. Wohlscin, M. Brgmann, T. Paul, G. Hausdorf

Pediatric Cardiology, Hannover Medical School, Hannover, D

The feasibility of and the biological response to potentially biodegradable devices were evaluated in the sheep model.

Percutaneous transcatheter closures of interventionally created atrial septal defects were performed in 20 lambs (body weight $32-44 \mathrm{~kg}$ ) using modified CardioSEAL septal occluders. In 14 devices, the springarm wire was replaced by a steel wire with high iron content (group I). Complete pathomorphological investigations and direct magnification radiography were carried out after 6,12 , and 18 months, respectively. In 6 animals, a device with a collagen layer instead of the dacron fabric was implanted for 2,4 , and 12 weeks (group II)

Mechanical stability and handling of all modified occluders during device implantation did not differ from standard occluders. All devices were covered by endothelialized granulation tissue as part 
if a normal healing response with formation of a neoendoardium depending on the follow-up intervals. Degradation and entral fractures of the springarm wire in group I could be lemonstrated radiographically and pathomorphologically, resultng in reduced mechanical stability of the explanted devices. The legradation products induced a moderate granulomateous inflamnatory reaction. In group Il, the collagen layers induced only a nild inflammatory response without cellular infiltration and are redominantly intact after 3 months.

n conclusion, metal as well as dacron parts of standard septal iccluders might be replaced by potentially biodegradable products rithout impairing their functional properties. Healing of the nodified devices is similar to standard occluders in the sheep nodel. These preliminary data support further experimental nvestigations of biodegradable implants.

35

Perventricular closure of muscular and perimembranous rentricular septal defects without cardiopulmonary sypass: application in animals and humans

J.A. Danford, J.M. Berry, J. Foker, J.L. Bass

'N/CU/Children's Hospital, Omaha, USA

Jbjectives: Cardiac catheterization laboratory (CL) closure of ventricular Septal Defects (VSD) is difficult in infants. Small size of the patient decreases the chance to successfully close the defect. jurgical closure is the gold standard, but requires cardiopulmonary yypass, which increases morbidity. This report describes the use of serventricular approach to close muscular VSD (MVSD) in uumans and, perimembranous VSD (PVSD) in a swine model in he operating room, without CPB. Amplatzer muscular and perinembranous VSD devices were used to close all defects.

Methods: A total of 4 patients (mean weight $6 \mathrm{~kg}$ ) underwent perventricular closure of MVSD. Three patients had failed attempt in he CL previously. After performing median sternotomy, and inder echocardiographic guidance, the free wall of the right venricle was punctured with an angiocatheter. A wire was advanced hrough the catheter and advanced across the VSD. The delivery sheath was advanced over the wire. The wire was removed, the device was advanced through the sheath, and deployed. Four Yucatan pigs with naturally occuring PVSD, had closure of PVSD using the same technique.

Results: Technical success rate was $100 \%$ in both groups. In the MVSD patients, follow up (range 0.9 to 3 years) revealed complete closure in 3 and trivial shunt in 1 . In the Yucatan pigs, there was complete closure at 6 months follow-up in all pigs. There was no aortic insufficiency.

Conclusions: The perventricular approach appears feasible in closing MVSD in infants. The results of PVSD closure in pigs are encouraging. Potential complications of $\mathrm{CPB}$ are avoided which reduces postoperative recovery time. Possible complications of CL and fluoroscopy are also avoided.

\section{6}

Transcatheter closure of perimembranous ventricular septal defects with the Amplatzer asymmetric ventricular septal defect occluder: preliminary experience in children G.S. Tsaousis, J. Bass, N. G. Eleftherakis, E. Karanasios,

E. Dragogia, B.D. Thanopoulos

Dept. Cardiology "Aghia Sophia" Children's Hospital, Athens, GR

The design of previously used devices for transcatheter closure of perimembranous ventricular septal defects (PMVSDs) is not ideal for this purpose and their use has been associated with several drawbacks. The aim of this study was to close PMVSDs in children using a new device the Amplatzer asymmetric ventricular septal defect occluder (AAVSDO). Eight patients, aged 2 to 11 years, with PMVSDs underwent attempted transcatheter closure using the AAVSDO. The device is a self-centering and repositionable occluder that consists of two low profile discs made of Nitinol wire mesh with a $1.5 \mathrm{~mm}$ connecting waist. The left-sided disc is $5 \mathrm{~mm}$ towards the apex and only $\mathrm{mm}$ towards the aortic valve. The right-sided disc is $4 \mathrm{~mm}$ larger than the waist. The prosthesis size (waist diameter) was chosen to be $2 \mathrm{~mm}$ larger than the angiographic PMVSD diameter. A 7F or an $8 \mathrm{~F}$ sheath was used for the delivery of the AAVSDO. Fluoroscopy and transesophageal echocardiography were used for the guidance of the procedure. Results: The angiographic PMVSD diameter ranged from 2.2 to $6 \mathrm{~mm}$. The device diameter ranged from + to $8 \mathrm{~mm}$. Qp/Qs varied between 1.5 to 2.2. Device placement was successful and associated with complete closure in all 8 patients. Two patients developed transient complete left branch block. One patient had an episode of non-sustained ventricular tachycardia. No other complications occurred.

Conclusions: The AAVSDO appears to be a promising device for the transcatheter closure of PMVSDs in children. Further studies are required to document its efficacy, safety and long-term results in a larger number of patients.

87

Is there a risk of thrombus formation on ASD and PFO closure devices?

U. Krumsdorf, S. Ostermayer, K. Billinger, T. Trepels, E. Zadan, K. Horvath, H. Sievert

Cardiovascular Center Bethanien, Frankfurt, $D$

Purpose: The purpose of this study was to investigate incidence, morphology and clinical course of thrombus formation on atrial septal defect (ASD) and patent foramen ovale (PFO) closure devices. Methods: In 813 consecutive patients (pts.) with a patent foramen ovale $(n=471)$ or an atrial septal defect $(n=342)$ transcatheter closure of the defect was performed. Routine transoesophageal echocardiography (TEE) was performed after 2-4 weeks and 6 months.

Results: Thrombus formation in the left $(\mathrm{n}=11)$ or right atrium $(n=6)$ or both $(n=3)$ was found in $5 / 342$ ASD pts. $(1.5 \%)$ and in $15 / 475$ PFO pts. (3.2\%). This was observed after $2-4$ weeks in 14 , after 6 months in 3 , after 1 year in 2 and after 5 years in 1 pt. The diameter of the thrombus varied from 5 to $30 \mathrm{~mm}(11.77)$. After 4 weeks the TEE revealed thrombus formation on the Cardioseal-occluder in 7\%, on the Starflex-occluder in 7\%, on the PFO-Star-occluder in $7 \%$, on the ASDOS-occluder in $4 \%$, on the Helex-occluder in $1 \%$, on the Sideris-occluder in $0 \%$ and on the Amplatzer-occluder in $0 \%$. The difference between the Amplatzer-occluder on one hand and the Cardioseal-occluder, the Starflex-occluder and the PFO-Star-occluder on the other hand was significant $(\mathrm{p}<0.05)$.

Prethrombotic disorders were found in 2 PFO pts. (in one thrombocytosis and in one hyperactivity of Factor VIII). Post procedure atrial fibrillation $(n=5)$ and persistent atrial septum aneurysm $\langle\mathrm{n}=5$ ) had been found as significant predictors for a thrombus formation $(p<0.05)$.

Two minor strokes caused by a left-sided mobile thrombus attached to a Starflex-occluder, one minor stroke and one TIA occurred in 2 PFO-Star pts. In 3 pts. the thrombus was removed surgically. $17 / 20$ pts. had a good outcome with disappearance of the thrombus following anticoagulation therapy with heparin or warfarin. 
Conclusion: The incidence of thrombus formation on closure devices is low. In most of the patients the thrombus resolves under anticoagulation therapy without clinical consequences. Surgery is rarely needed and should not be recommended generally.

\section{8}

Animal study of a reexpandable "growth stent" for the treatment of vessel stenosis in infants and small children P. Ewert, E. Riesenkampff, M. Neuss, O. Kretschmar, H. Abdul-Khaliq, N. Nagdyman, P.E. Lange

German Heart Institute, Berlin, D

In small children stent implantations are problematic, because the stents are often not potentially expandable to the size needed for the corresponding adult vessel. Thus, these stents may cause undilatable stenosis when the child grows.

Methods: We evaluated a stent made of two longitudinal halves. These were fixed to each other by resorbable sutures, so that the two halves could be separated from each other after resorption of the sutures, enabling unlimited vessel growth.

We implanted 17 stents in 11 piglets (age $5 \mathrm{wks}$, weight $6-8.5 \mathrm{~kg}$ ) into the descending aorta $(\mathrm{n}=6)$ and the pulmonary arteries ( $\mathrm{n}=11$ ). Prior to sacrificing of the animals after 12 to 16 weeks (weight $42-89 \mathrm{~kg}$ ), re-catheterizations were performed and stents redilated, if indicated. The stented vessels were examined histologically.

Results: Implantation was possible through $5 \mathrm{Fr}$ sheaths and was comparable to treatment with conventional stents. After 12 to 16 weeks a hemodynamically significant stenosis (pressure gradient $>5 \mathrm{mmHg}$ ) could be ruled out in every animal. However, mild morphological stenosis was detected in 5 vessels. In 3 of these cases dilation beyond the diameter of the proximal vessel was possible. Conclusion: The "growth stent" seems to have the potential to be implanted in early childhood without causing restriction during the patient's lifetime. However, long-term results with regard to intimal proliferation are lacking.

\section{Poster Presentations}

\section{P100}

Feasibility and safety of automatic capture control in permanent epicardial pacing in children

J. Janousek, $P$. Vojtovic

Kardiocentrum, Lniv. Hosp. Motol, Prague, CZR

Data on feasibility and safety of automatic capture control in permanent epicardial pacing in children are limited.

Methods: Pulse generators with the AutoCaptureTM feature (Entity $\mathrm{DC} / \mathrm{DR}, \mathrm{N}=13$; Affinity $\mathrm{DR}, \mathrm{N}=2$; Regency SR, $\mathrm{N}=1$; St. Jude Medical, Inc.) were implanted in 16 consecutive pts. (preceding or concurrent cardiac surgery in $13 / 16$ ) aged $1 \mathrm{~d}$. $12.6 \mathrm{y}$. (median $4.3 \mathrm{y}$.) and connected to bipolar ventricular epicardial steroid eluting leads CapSure Epi $4968(\mathrm{~N}=15)$ and 10366 $(\mathrm{N}=1)$; Medtronic, Inc. Pts. were followed-up 1,3 and every $6 \mathrm{~m}$. after implantation for a median of $5.2 \mathrm{~m}$. (19 d. $-36 \mathrm{~m}$.). Evoked response (ER) sensitivity was programmed to $50 \%$ of actually measured ER amplitude.

Results: AutoCaptureTM could be used 14/16 pts. during the follow-up period. In $2 / 16$ pts. evoked response signal was to low or resting heart rate to high to enable capture verification or automatic threshold search, resp. Mean (SD) ER signal $(15.66 .6 \mathrm{mV})$, lead polarization $(0.80 .4 \mathrm{mV})$ and threshold at $0.4 \mathrm{~ms}$ pulse duration $(1.00 .5 \mathrm{~V})$ did not differ significantly between implantation and subsequent follow-up. Mean (SD) maximum decrease in ER signal amplitude between 2 subsequent follow-up evaluations was 20.6 $15.0 \%$, exceeding $50 \%$ and leading to ER undersensing and high output pacing in $1 / 14$ pts. Lead polarization increased to a maximum of 15.9 (mean 6.03 .2 ) \% of ER amplitude measured at preceding follow-up and did not lead to false positive capture detection in any of the 14 pts. Using automatic pulse amplitude adaptation, theoretical generator longevity (mean SD) increased from 13419 to $1559 \mathrm{~m}$. compared to pacing at twice voltage threshold.

Conclusion: The use of automatic capture control by AutoCapture TM is feasible and safe in connection with steroid eluting epicardial pacing leads in children and leads to increase in theoretical pacemaker longevity.

\section{P101}

Cardioverter/defibrillator therapy in children

A. Eicken, S. Lange, Ch. Koll, B. Zremer, C. Schmitt,

S. Brodherr, J. Hess

Department of Pediatric Cardiology and Congenital Heart Dise, München, $D$

Introduction: The indication for cardioverter/defibrillator (ICD) implantation is well defined(Gregoratos et al Circulation 1998). In contrast to adults children often do not present with a normal cardiac anatomy, do not attain adult height and are not able to consciously decide on ICD implantation. This study was undertaken to investigate the results of ICD-implantation in children. Methods: Since November 1995 ten patients (f:2, m:7) received an ICD. Mean age was 11.3 years, range $5.8-15.9$ years. Three pts had complex congenital heart defects (DORV after "Kawashima" operation), two had long QT syndrome, one patient had neonatal PS (after comissurotomy), one had congenital AV block III with ventricular tachycardia (VT) and three had no structural heart disease. Eight of these children had syncope with documented ventricular fibrillation in 7 cases and a VT in one case. The two other children had sustained VT with hemodynamic significance. All ICD leads were implanted transvenously.

Results: Mean follow up was 14.6 months, range 1-68 months. So far all pts are alive. Thirty six episodes of ventricular fibrillation were terminated by defibrillation and 186 VT's were ended by overstimulation. In five pts the implanted ICD's did not show activity. Three of these pts had a positive biopsy for viral mvocarditis. There were two incidents of $\mathrm{T}$-wave oversensing with inadequate shocks in 2 pts. In both the leads had to be replaced $t$ and 68 months after inital implantation.

Conclusion: ICD-Implantation with transvenous leads is possible and effective in children. T-wave oversensing and ICD-activity during sinus tachycardia do occur. Data on the impact of ICD implantation on life quality of these patients will be presented.

\section{P102}

Age-related differences of direct cardiac effects of cisapride: a narrower safety range in the young hearts M-H. Wu, M-J. Su, S. Siu-Man Sum

National Taiwan Lniversity Hospital, Taipei, TAI

Cisapride is widely used to treat the gastrointestinal motility disorders. But, it has been associated with QT prolongation, torsades de pointes and cardiac arrest. Only in children, atrioventricular block after cisapride had been described. This study by using the Langendorff-perfusion defined the direct effects of cisapride $(0.03,0.1,0.3$ and $1 \mathrm{mM})$ on conduction properties in the neonatal ( $<7$ days) and adult ( $>3$ months) rabbit hearts. At clinically relevant dose $(0.03 \mathrm{mM})$, cisapride slowed down the recovery of the His-Purkinje system. At $0.1 \mathrm{mM}$, the refractoriness of 
Iis-Purkinje system and the conduction through this system were rolonged. Corrected $Q T$ intervals and ventricular refractory eriod were also lengthened. The degree of prolongation of these arameters was significantly nore in the neonate than in the adult. The level of atrioventricular block at rapid atrial pacing shifted :om the AV node to the His-Purkinje system with an ED50 0.06 nd $0.52 \mathrm{mM}$ in the neonate and the adult, respectively. In the teonate, cisapride even resulted in infranodal atrioventricular lock rhythm (EI)50 $=0.12 \mathrm{mM}$ ), but not in the adult. 'olymorphic ventricular tachycardia after cisapride occurred in ine out of seven adult hearts $(14 \%, 0.03 \mathrm{mM})$ and one out of even neonates $(14 \%, 0.1 \mathrm{mM})$. Therefore, it is concluded that isapride may affect the refractoriness of cardiac tissue with the ighest sensitivity in the His-Purkinje system. In neonatal hearts, his modification may progress to infranodal atrioventricular lock. Such susceptibility to cisapride indicates a narrower therareutic safety range in the young hearts.

\section{$>103$}

Jompatibility of automatic threshold tracking pacemakers vith previously implanted pacing leads in children

J. Kucukosmanoglu, A. Celiker, S. Ozer, T. Karagoz

Hacettepe Lniversity, Ankara, TR

The Autocapture function controls and optimizes the amplitude ff pacing pulse and provides energy saving. The manufacturer St. Jude-Pacesetter) recommends using a special low polarization, ow threshold bipolar Pacesetter lead for Autocapture function. The purpose of our study was to evaluate the compatibility of 4utocapture with previously implanted non-recommended sacing leads. The study included 15 patients (mean age 13,6 +3,4 .ears) who needed pulse generator replacement and received the VVIR pacemaker Regency SR+ or the DDDR pacemakers Affinity DR or Integrity DR with Autocapture function. The new sulse generators connected to previously implanted ventricular leads. At the time of implantation the pacing threshold was $1.0+0,35 \mathrm{~V}$ at $0,5 \mathrm{~ms}$, the lead impedance was $580+80 \mathrm{ohms}$, and the spontaneous $R$ wave amplitude was $7,89+4,89 \mathrm{mV}$. The polarization signal (PS) was $3,8+3,04 \mathrm{mV}$, and evoked response (ER) was $8,15+4,57 \mathrm{mV}$ at the predischarge testing. Follow-up telemetry was done at months $1,3,6,12$, and 18 . The follow-up duration was $9,4+5$ months (ranging in 1 to 18 months). If the results of PS and ER measurements were acceptable for Autocapture, it turned on at the 1 -month visit. In six patients, (40\%) the results were found acceptable for Autocapture function. Age, lead impedance, pacing threshold, intrinsic $\mathrm{R}$ wave measurement, lead age, fixation mechanism, and ER measurements were not statistically different in Autocapture suitable and not suitable groups. The main reason not to activate Autocapture has been increased PS. Any significant fluctuations were not observed in pacing threshold, lead impedance, ER, and PS during follow-up period. In conclusion, previously implanted non-recommended pacing leads may be compatible with Autocapture function.

\section{P104}

AutoCapture device obtained evoked response and polarisation signals

L. Bauersfeld, O. Przibille, T. Fhraeus, M. Rahm, H. Schller

Lniversity Children's Hospital, Zurich, CH

Although the energy saving and safety of AutoCapture technology has been demonstrated there is little information about the long-term stability of evoked response (ERS) and polarisation (PS) signals.
Methods: In 87 children aged 7266 months an AutoCapture device (Microny, Regency, Affinity, Integrity, St.Jude Medical) was implanted. ERS and PS were obtained from 78 patients $(52$ epicardial (epi) leads Medtronic Capsure 10366 or 4968, 26 various endocardial (endo) leads) at discharge (1)/C) and every 6 months thereafter.

Results: During 1813 months follow-up a decline of ERS that precluded AutoCapture controlled pacing was observed in $3(6 \%)$ epicardial but no endocardial leads. Significant changes of PS were not observed.

Table

${ }^{\star} \mathrm{p}<0.05, \mathrm{p}=0.0005$ vs endo, $\# \mathrm{p}<0.05$ vs endo

Conclusion: Although there is a significant decrease in ERS of epicardial leads during long-term follow-up ERS and PS still allow AutoCapture controlled pacing. Rarely epicardial leads show a decline of ERS which precludes AutoCapture controlled pacing. PS of the investigated epicardial leads are significantly higher than those of the various endocardial lead models implanted in our study

\section{P105}

AutoCapture device obtained pacing threshold trends

L: Bauersfeld, O. Przibille, T. Fhraeus, M. Raln, H. Schweller

Liniversity' Children's Hospital, Zurich, $\mathrm{CH}$

Conventionally pulse generator output is programmed with a 2-3 fold safety margin. AutoCapture devices stimulate only slightly above the actual threshold and provide threshold trends over time. Methods: In 51 children aged 8366 months an AutoCapture device (Microny, Regency, Affinity, Integrity, St.Jude Medical) was implanted. Epicardial (epi) leads (Medtronic Capsure 10366 or 4968) were used in 32 and various endocardial (endo) leads in 19 children. Device stored stimulation threshold trend curves were obtained 1, 3, 6 and every 6 months after device implantation. Differences between maximum and minimum voltage $(\star \mathrm{V})$ at a given pulse width $(\mathrm{PW})$ and pulse energy ( $\left.{ }^{\star} \mathrm{PE}\right)$ were calculated.

Results: During 1912 months follow-up a threshold trend to the maximum of $4.5 \mathrm{~V}$ was observed in 3 children with epicardial leads and was due to evoked response undersensing in 2 and real threshold rise in 1 child. Significant $\star V$ and $\star P E$ differences between epicardial and endocardial leads were only observed at 12 months follow-up.

Table

${ }^{\star} \mathrm{p}<0.05$ epi vs endo

Conclusion: Epicardial and endocardial leads show little pacing threshold variations during long-term follow-up with no significant trend variations between epicardial and endocardial leads. Real pacing threshold rises are rare with modern pacing leads.

\section{P106}

Implantable cardioverter defibrillators in pediatric patients: experience in 7 cases

B. Hennig, F. Heinke, H.J. Husler; I. Dhnert, P. Schneider, H. Kottkanp*

Dept. of Paediatric Cardiology and Cardiology ${ }^{\star}$, Leipzig, D

Objective: Implantable cardioverter defibrillators (ICD) represent an important treatment option for patients with life-threatening tachyarrhythmias. However, the experience regarding the optimal choice of ICD and lead system in children and adolescents is limited. 
Methods: The medical records of 7 children and adolescents with implantable cardioverter defibrillators were retrospectively reviewed. Underlying pathology included arrhythmogenic right ventricular dysplasia, hypertrophic cardiomyopathy, idiopathic ventricular flutter, Emery-Dreifuss muscle dystrophy, ventricular arrhythmia after remote repair of congenital heart disease and long QT syndrome in 2 patients (age 12-18 years, median 15,3). A nonthoracotomy approach and the placement of a transvenous defribillator lead system was feasible in all cases. Due to a high defibrillation treshold one patient required addition of a subcutaneous patch to achieve adequate defibrillation treshold.

Results: There were no postoperative complications and no deaths during the follow-up (9-40 months, median 20,4). During this time 4 of 7 patients received at least one ICD discharge. The leading complication was one or repeated inappropriate defibrillator therapy in 3 patients due to detection of supraventricular tachycardias. The freqency of these events was clearly lower with additional beta blocker therapy.

No lead dislocation occured during the follow up.

Conclusion: The nonthoracotomy approach and transvenous lead placement is possible with a low complication rate in pediatric patients and may be particularly advantageous for patients who have undergone prior thoracotomy. The incidence of inappropriate ICD discharge can be lowered with medical therapy (beta blocker).

\section{P107}

\section{Combined intervention and radiofrequency ablation for} arrythmias in patients with congenital heart disease J.V. De Giovanni, J. Vettukattil, P. Miller, M. Griffith, D. Wilson Heart Unit, Birmingham Children's Hospital, Birmingham, UK

Arrhythmias can co-exist with structural heart disease or they can be iatrogenic following cardiac surgery. Arrhythmia therapy using radiofrequency (RF) has advanced over the past 10 years and catheter intervention has reached new heights due to advanced technology. We describe combined procedures in a group of patients who had arrhythmias as well as a structural defect, both of which were in need of treament; these procedures were carried out during the same session.

Seven patients were treated with combined procedures, 5 were female and 2 male. Their ages ranged from 4 to 26 years (Mean 14.8). The heart defects consisted of Ebsten's anomaly and Atrial Septal Defect in 2, Superior vena cava obstruction following Mustard's operation for Transposition in 2, Islated Atrial Septal Defect in 2 (one with concomitant PDA) and 1 patient with pulmonary atresia who had a coronary fistula.

The arrhythmias that were ablated consisted of atrial flutter in 2 , AV node re-entry tachycardia in 2 and accessory pathways in 4 . The interventional procedures consisted of stent implantation in 2, coil closure for PDA in 1, atrial septal defect closure using Amplatzer ASO device in 3 and coil embolisation of coronary fistula in 1.

There were no complications or deaths but 1 patient with flutter developed a recurrence but was easier to control. One patient with a left sided accessory pathway and ASD had the 2 procedures separately.

Conclusion: Combined RF ablation and Intervention are safe, feasible and desirable. It provides good quality care and it also minimises vessel trauma; it is of particular benefit when vessel access is limited because of congenital anomalies of the vessels or because of previous cannulation.

\section{P108}

Mental stress, different body positions and exercise seem to have no clinically significant influence on the autocapture function

T. Karagoz, A. Celiker

Hacettepe University Faculty of Medicine, Ankara, TR

The autocapture function controls the evoked response signal and lead polarization for the calculation of the appropriate evoked response sensitivity setting and optimizes the output of the ventricular pulse amplitude automatically during follow-up. The present knowledge about the autocapture function lability in some patients is very limited. The purpose of this study was to evaluate whether body position, exercise or mental stress influence the evoked response and polarization signal. The autocapture functions were measured in different body position including supine, sitting and standing, and during mental stress test, and during symptom-limited treadmill exercise test, and during recovery of both tests. A mental arithmetic stress test was applied in all except two patients $(5$ and 8 year of age) who did not have ability to perform this test. The study was performed in 15 patients (mean age $13.44 .1 \mathrm{yr}$, range $5-20 \mathrm{yr}$ ) with the VVIR single-chamber pacemakers $(n=10)$ and the DDD or VDD dual-chamber pacemakers $(n=5)$, who had received ventricular pacing leads including Membrane E $1450 \mathrm{~T}(\mathrm{n}=6)$ and EX 1470T $(\mathrm{n}=2)$, Tendrill DX $1388 \mathrm{~T}(\mathrm{n}=3)$ and Tendrill SDX $1488 \mathrm{~T}(\mathrm{n}=1)$, AV Plus DX $1368(\mathrm{n}=1)$, Telectronics Accufix II DEC $(n=1)$ and Vitatron $(n=1)$. The pacemaker did not recommend activating autocapture in only one patient with Telectronics Accufix II DEC ventricular lead due to high polarization signal. The evoked response signal was $10.56 .3 \mathrm{mV}$ during supine and increased to $11.97 .5 \mathrm{mV}$ during sitting $(\mathrm{p}=0.017)$ and standing 12.17.2 $(\mathrm{p}=0.002)$. However, the evoked response signals remained stable just before, during and after both exercise and mental stress test, which were $12.67 .2 \mathrm{mV}, 12.87 .8 \mathrm{mV}, 13.69 .4 \mathrm{mV}$ $(\mathrm{p}>0.05)$ and $10.55 .5 \mathrm{mV}, 10.96 .7 \mathrm{mV}, 10.45 .5 \mathrm{mV}(\mathrm{p}>0.05)$ respectively. In addition, polarization signals remained unchanged during different body positions, exercise test in upright position and mental stress test in sitting position. As a result recommendation about autocapture did not change during the study. In conclusion, although the evoked response signal was significantly low in supine position, the autocapture function did not effected by different body positions, exercise and mental stress even in patients with unrecommended pacing leads.

\section{P109}

Do not worry about long-term isolated pulmonary

insufficiency: it may never lead to symptomatic RV dilatation

Y. d'Udekem, A. Pasquet, L. Lebreux, C. Onaert, C. Barrea,

P. Shango-Lody, M. Cailteux, T. Sluysmans, JE Rubay, Ph. Noirhomme

Ciniques Universitataires St-Luc, Brussels, $B$

Background: To determine the relative role of pulmonary insufficiency and right ventricular (RV) outflow tract damage in the genesis of the late symptoms related to RV dilatation, we compared the late outcome of patients who had undergone operations known to generate pulmonary insufficiency: transventricular repair of tetralogy of Fallot and isolated pulmonary commissurotomy. Methods and results: In our institution, between 1964 and 1984. 44 pts had an isolated pulmonary commissurotomy and 189 survived a transventricular repair of tetralogy of Fallot: 134 had a patching of the RV and 55 a direct closure of a right ventriculotomy. Follow-up was $94 \%$ complete after a mean of 227 years. On echocardiography, patients with isolated commissurotomy had 
imilar proportion of moderate and severe pulmonary insuffiiency than Fallot patients who had a right ventricular patch $p>0.2$ ). Cardiac death, reoperation for symptoms due to RV lilatation, and NYHA class 2 were investigated as adverse events elated to RV dilatation. Among the pts who had a pulmonary ommissurotomy, there were no cardiac death or reoperation. -he freedom from all adverse events was far better for pts with an solated pulmonary commissurotomy than for pts with a direct losure of the RV (log rank $\mathrm{p}=0.02)$ or a RV patch (log rank ,$<0.001$ ). In pts with an isolated commissurotomy, the ratio etween end-diastolic right and left ventricular dimensions was maller than in pts with a RV patch $(0.560 .14$ vs 0.710 .24 , $,<0.001)$ and similar to the ratio of the pts with direct closure of right ventriculotomy (0.600.17, $\mathrm{p}=0.33$ ).

Conclusion: Pulmonary insufficiency may not be the key factor eading to symptonatic RV dilatation in pts operated of tetralogy if Fallot. Long-term pulmonary insufficiency alone is responsible or slight degree of $\mathrm{RV}$ dilatation but provided that the pts have ormal pulmonary arteries, symptoms may never develop if the ontractility of the pulmonary infundibulum is preserved

\section{$>110$}

?acemaker and lead revision in children with ransvenous pacemaker

4. Eliker, G. Kafali

Hacettepe Lniversity Medical Faculty Department of Pediatric, 4nkara, TR

Jbjective: Transvenous cardiac pacing has become standard pracice for children who require pacing therapy. During long-term ollow-up many of these children may require revision or explanation of the pacing system and leads. Our aim was to evaluate he results of pulse generator and/or lead revision-extraction in shildren with pacemaker.

Material and methods: Between February 1992-October 2001, $59(37 \%)$ of one hundred fifty-eight children underwent redo sacemaker intervention (a total of 233 procedures). The indicaions for pacing were complete atrioventricular block in most of he patients. Passive fixation leads were used in 30 patients (51\%) and active fixation leads in $29(49 \%)$ patients. They were firstly slaced into the right ventricle in 49 , into the right atrium in ight and into the both chamber in two patients.

Thirty-four pulse generator removal procedures were performed in 32 patients due to end of battery life and infection at a median seriod of 72 months. A total of 41 lead required removal in 36 patients. These leads had been implanted for a median period of 30 months (range 1-120 months). In 14 of these patients, the both sulse generator and lead removal procedure were made. Pacemaker explantation was performed in six of 14 patients because of recovery of atrioventricular conduction. In the remaining 8 patients, pulse generator and lead revision procedure were performed. Median age of the patients during lead removal were 12,5 years (range $2,5-18,5$ years). Thirty-six of 41 leads $(88 \%)$ were removed by simple traction method through subclavian vein. The different traction systems (Cook traction system and VascoMed traction system) were used in 3 leads. Abandoned passive lead fragments of two patients was removed by gooseneck snare catheter through femoral vein. Three passive and one active fixation type leads could not extracted and they were retained in the subclavian vein. Lead removal was successful in $37(90 \%)$ of 41 leads.

Conclusion: Pacemaker replacement and lead extraction can be safely and effectively performed in children. In most patients simple traction methods would be satisfactory. However several extraction tools may be used in difficult cases.
P111

Impaired atrio-ventricular conduction in the long QT syndrome: a functional response to extreme prolongation of ventricular refractoriness B. Sarubbi, M. D'Alto, B. Mercurio, R. Calvanese, P. De Gregorio, M. Carrozza, R. Calabr Pediatric Cardiology-Monaldi Hospital-SUN, Naples, I

Background: The coexistence of QT prolongation, torsade de pointes type ventricular tachycardia and impaired AtrioVentricular conduction represents a malignant variant in infants, potentially leading to sudden death. The purpose of the study is to examine the clinical presentation, and outcome of a cohort of 5 pts, all medically treated, with congenital long QT syndrome and impaired AV conduction referred in the period between January 2000 and March 2001 to our Division.

Material and methods: Five pts ( $4 \mathrm{M} / 1 \mathrm{~F})$, referred to our Division within the first week of life for occasionally detection of long QT (>460 ms) and/or life-threatening ventricular tachycardia entered the present study as they showed on the standard ECG and/or 24-hour ECG monitoring different degrees of impaired AV conduction. All the pts underwent medical treatment.

Results: No pts had a familiar history of syncope, long QT syndrome or sudden death. No patients showed major congenital heart defect, but patent ductus arteriosus (4 pts) and/or trivial atrial septal defect (2 pts). During the continuous ECG monitoring through ECG Holter or Telemetry, three patients showed episodes of lifethreatening ventricular tachycardia (mainly torsade de pointes). All the patients showed different degrees of impaired AV conduction, before the use of any antiarrhythmic drug therapy. Two of the patients with the torsades de pointes showed 2:1 AV block, the third showed Wenckebach episodes. The other two patients had episodes of impaired right or left bundle branch conduction. All the patients underwent Propranolol treatment $(1-6 \mathrm{mg} / \mathrm{kg} /$ day per os) with complete disappearance of life-threatening ventricular tachycardia and $\mathrm{AV}$ conduction disturbances in the follow-up (3-10 months). Conclusion: Conduction abnormalities associated with long QT syndrome have to be considered as "pseudo" AV block due to sinus intervals shorter than ventricular refractoriness. The use of beta-blockers, reducing the extreme prolongation of ventricular action potential duration, can be safety used in long QT syndrome with different degrees of AV conduction impairment.

\section{P112}

\section{Long-chain 3-hydroxyacyl-coenzym a dehydrogenase deficiency (LCHADD) rare reason of long QT M. Zuk, J. Rekauek, W. Kawalec, M. Pohorecka, E. Pronicka Children's Memorial Health Institute, Warsaw, $P L$}

Long-chain 3-hydroxyacyl-coenzym A dehydrogenase (LCHAD) deficiency (LCHADD) is an inherited metabolic disorder diagnosed with increased frequency. A clinical picture of LCHADD includes myalgia, hypotonia, weakness, hepatomegaly, hepatic steatosis, pigmentary degeneration of retina, Reye-like syndrome, SIDS and cardiomyopathy, but these symptoms may be present only during catabolic crisis. QT elongation has never been reported before in patients with LCHADD.

The study was performed in 11 LCHADD children. Echo, 12-leads ECG and Holter monitoring were performed in all patients, treadmill test only in one girl older than 5 years with recognized long QT. QT elongation was observed periodically in 5 patients (45\%) in acute life threatening episode (ALTE) and/or in remission. One of them had dilated cardiomyopathy, two had pericardial effusion (severe, needed pericardiotomy and mild), but when the additional 
symptoms disappeared long QT was still present. Children with long QT are on propranolol. Benign ventricular arrhythmia was observed in 1 girl with long QT before beta-blockers therapy. Maximum QTc was 0,52 at ALTE, when she also had pericardial effusion. Now this girl is during remission, still on propranolol, normal in echo. She is without ventricular arrhythmia (in Holter and in treadmill test), but periodically with long QT despite therapy (max QTc 0,48). Ventricular tachycardia (during Holter monitoring) was the cause of death of one other patient (not reported above as a patient with long QT). Echo was normal. 12-leads ECG performed before was without long QT, but he has never had Holter monitoring previously. We could suspect that QT elongation might be a reason of fatal arrhythmia.

Summary: LCHADD may be the rare reason of recurrent long QT. In patients with long QT with unknown etiology metabolic screening should be performed.

\section{P113}

\section{1 years experience of epicardial pacing in}

the paediatric population

J.D.R. Thomson, M.E.C. Blackbum

Department of paediatric cardiology, Leeds, $U K$

Aims: To audit a single centre experience of epicardial pacing in children over 2 decades, specifically looking at system and lead survival and risk factors for failure of both.

Patients: A total of 95 leads ( 6 atrial and 89 ventricular) and 90 generators in 59 patients were implanted between $6 / 1980$ and 8/2001. Median age at implantation was $2.76 \mathrm{yrs}(1 \mathrm{~d}-18 \mathrm{yrs})$. Indications for pacing were congenital complete heart block (37\%), "other" higher degrees of heart block (20\%), post oper-ative heart block $(37 \%)$ or bradycardia related to antiarrhythmic therapy (5\%). 26/59 (44\%) patients had structural heart disease.

Results: Follow up data was available in all patients. A mean of 1.6 pacing leads and 1.5 generators were implanted per patient with total follow up of 326 patient years. $7 / 59$ (12\%) (95\% CI 2.1-11.9) patients died with only 1 death due to possible pacemaker failure. Overall system survival was 80,69 and $60 \%$ at 1,3 and 5 years respectively with mean time to system failure of 3.4 yrs (SD 3.27). Causes of system failure were lead fracture $(50 \%)$, exit block $(27 \%)$, lead displacement $(8 \%)$ and generator end of life $(15 \%)$ (mean generator survival time $4.7 \mathrm{yrs}$ ). Lead survival was 83,71 , and $62 \%$ at 1,3 and 5 years (mean time to failure 3.1 yrs SD 3.3). By multivariate analysis the only significant risk factor for lead failure was earlier decade of implant $(\mathrm{p}<0.03)$. There was a trend towards steroid eluting leads surviving longer than non steroid eluting leads $(p=0.08)$. Site of pacing wire (atrium or ventricle) and presence of congenital heart disease were not significant risk factors for lead or system failure.

Conclusions: Epicardial pacing has offered an effective solution to pacing in the paediatric age range over 2 decades and remains our preferred mode of pacing in order to avoid vascular complications associated with endocardial systems.

\section{P114}

The role of TGFbeta during cardiac morphogenesis: distinct expression patterns of TGFbeta1-3 mRNA and characteristic effects of TGFbeta2-knockout in the embryonic mouse heart

U. Bartram, D. G.M. Molin, K. van der Heiden, C.P. Speer,

T. Doetschman, A.C. Gittenberger-de Groot

University Children's Hospital Wraburg, Wrzburg, D

Background. There is increasing evidence for the importance of the transforming growth factor beta (TGFbeta) in normal cardiac morphogenesis and the pathogenesis of congenital heart disease.
Material and methods. The expression patterns of TGFbeta1-3 mRNA during normal cardiac development were studied in mouse embryos from 9 to 15 days post coitum using in-situ-hybridization. Cardiovascular malformations in TGFbeta2 knockout embryos (11.5-18.5 days post coitum) TGFbeta2 knockout embryos were generated and processed immunohistochemically to study the type of cardiovascular malformations and determine which processes of cardiac morphogenesis are affected by the absence of one isoform. Results. TGFbeta 1 was mainly associated with the inner lining of the developing heart tube. At the time of cushion formation TGFbeta? was present in the endocardium and the underlying myocardium. Epithelial-mesenchymal transformation was associated with dis appearance of the endocardial signal and expression in the mesenchyme which persisted during cushion differentiation. Cardiac expression of TGFbeta 3 started at day 12 and showed a characteristic distribution in the cushions suggesting an additional role in cushion differentiation. TGFbeta2 knockout resulted in malformations of the outflow tract $(87.5 \%)$ and AV canal region $(62.5 \%)$. Anomalies of the aortic branches and semilunar valve thickening were seen in $33.3 \%$ and $4.2 \%$, respectively. Immunohistochemical staining showed failure of myocardialization of the mesenchyme of the atrial septum and the ventricular outflow tract as well as deficient valve differentiation. Conclusions. All 3 isoforms of TGFbeta exhibited distinct expression patterns during normal cardiac morphogenesis. Heart defects in TGFbeta2-null mice showed a characteristic spectrum which correlated well with the structures normally expressing this isoform. These findings support the idea of different, but partially overlapping functions of the TGFbeta isoforms.

\section{P115}

Troponin- $T$ as a predictor for adriamycin-induced cardiomyopathy in rats

E. Koh, T. Nakamura

Kanazawa Medical Lniversity Deparment of Pediatrics, Ishikatua,J

Purpose: To investigate whether ANP, BNP, and Troponin-T (TnT) are predictors for Adriamycin-induced cardiomyopathy (ADR-CM).

Material and methods: ADR-CM was induced in Wister rats by weekly administration $2 \mathrm{mg} / \mathrm{kg}$ of ADR via tail vein for 8 weeks (ADR group). As a control group. physiological saline was injected into rats by same protocol. Serum ANP, BNP, and TnT were serially quantified $6,8,10,12$ weeks after the first administration of ADR and simultaneously echocardiography was performed. The correlations between LV performance and the serum concentrations of ANP. BNP, and TnT were studied.

Result: LV performance (\% fractional shortening) gradually diminished from 6 weeks to 12 weeks after ADR first injection (58.6. 57.750 .9 , and $36.8 \%$, respectively,). TnT increased gradually from 6 weeks to 12 weeks after the ADR first injection $(<0.01$, $0.02,0.10$, and $1.09 \mathrm{ng} / \mathrm{ml}$, respectively), with the deterioration of LV contractility. BNP also increased from 6 weeks to 12 weeks after ADR first injection (81.5 to $173.3 \mathrm{pg} / \mathrm{ml}$ ). ANP wasn't different from control at each week

Conclusion: These results showed that the measurement of the serum concentration of TnT was useful to predict ADR-CM.

\section{P116}

Impaired recovery from hypoxia of the isolated perfused heart following neonatal hypoxia in the rat C.V. Rollicek, S. Viau Montréal Children's Hospital/Me Gill Linversity, Montréal, CD.N

We have asked whether chronic hypoxia neonatally $(\mathrm{FiO} 2=$ (1.12, days $1-10$ of life) in the rat has long-lasting effects on the 
ardiac response to acute hypoxic stress in later life. Isolated hearts if 48 adult Sprague-Dawley rats $(4+87 \mathrm{~g}, 1024 \mathrm{~d}$ (SEM)) were tudied. Twenty-four animals experienced hypoxia neonatally Neonatally Hypoxic) while 24 others did not (Control). The hearts vere perfused in the Langendorff mode with measurement of left entricular pressure (LVP), maximum rate of pressure increase $+\mathrm{dP} / \mathrm{dtmax}$ ) and total coronary flow (CorFlow). Twelve hearts rom each group were perfused at a constant pressure of $00 \mathrm{cmH} 2 \mathrm{O}$ (ConstPress) and 12 others perfused at a constant low (ConstFlow) set to $120 \%$ of the CorFlow under ConstPress $t$ the outset of the experiment. The hearts were perfused with: ) oxvgenated perfusate $(95 \% \mathrm{O} 2+5 \% \mathrm{CO} 2)$ for 30 minutes, I) with hypoxic perfusate $(10 \% \mathrm{O} 2+5 \% \mathrm{CO} 2+85 \% \mathrm{~N} 2)$ for 30 ninutes, III) again for 30 minutes with oxygenated perfusate. At raseline there was no difference between Neonatally Hypoxic and Zontrol groups in LVP and $+\mathrm{dP} / \mathrm{dtmax}$ under ConstPress or ZonstFlow: Perfusion with hypoxic perfusate led to similar lecreases in LVP and $+\mathrm{dP} / \mathrm{dtmax}$ in both groups. However, inder ConstPress reoxygenation was associated with impaired ecovery of LVP and $+\mathrm{dP} / \mathrm{dtmax}$ at 30 minutes in the Neonatally typoxic hearts compared to Controls $(725 \%$ vs. $917 \%$ of baseline, $725 \%$ vs. $947 \%$ of baseline $\mathrm{P}<0.05$ ). Although CorFlow under ZonstPress was the same at baseline in Neonatally Hypoxic and Zontrol hearts ( 5.30 .3 vs. $4.90 .3 \mathrm{ml} / \mathrm{min} / \mathrm{g}$ ) it was less during perusion with hypoxic perfusate in the former than in the latter learts $(6.50 .4$ vs. $8.40 .5 \mathrm{ml} / \mathrm{min} / \mathrm{g}, \mathrm{P}<0.05)$ and in contrast to he Controls decreased to significantly less than the pre-hypoxic evel after 30 minutes of perfusion with re-oxygenated perfusate 3.60 .2 vs. $4.60 .2 \mathrm{ml} / \mathrm{min} / \mathrm{g} \mathrm{P}<0.05)$. In hearts perfused at ConstFlow there was no difference between Neonatal Hypoxia and Control groups in the recovery of LVP and $+\mathrm{dP} / \mathrm{d}$ tmax upon rexygenation ( $991 \%$ vs. $943 \%$ of baseline, $914 \%$ vs. $942 \%$ of baseine at $30 \mathrm{~min}$.). These findings suggest a long-lasting cardiac vulnerability to hypoxic stress after chronic hypoxia neonatally due so impaired coronary vasodilatation during hypoxia and on reoxygenation.

\section{P117}

Effects of acute left ventricular unloading on right ventricular function in normal and chronic right ventricular pressure overloaded lambs

B.P.J. Leemuenburgh, W.A. Helbing, P. Steendijk, P.H. Schoof, J. Baan Leiden Linversity Medical Center, Leiden, $N L$

Background: Right ventricular (RV) pressure overload occurs in several types of (congenital) heart disease as well as in pulmonary disease. Clinical outcome in some of these groups depends in part on left ventricular (LV) loading conditions. The effects of LV unloading by total left heart bypass on RV function have not been studied for the hypertrophied RV. We aimed to study the effects of LV unloading on RV hemodynamics and contractile state in an animal model of chronic RV pressure overload at systemic (aortic) level.

Methods: In lambs, the pulmonary artery was chronically banded to increase RV pressure to systemic levels using a bi-directionally adjustable pulmonary artery constrictor. After 8 weeks, RV contractile state and hemodynamic function were assessed in these lambs as well as in age-matched controls using a combined pressure-conductance catheter in the RV during baseline conditions and during complete bypass of the LV.

Results: In the control group, acute LV unloading decreased LV end-systolic pressure from 7732 to $2938 \mathrm{mmHg}(\mathrm{p}<0.01)$. Both end-systolic and end-diastolic volumes in the RV were increased during LV unloading $(p<0.01)$ while RV end-systolic pressure was maintained. Cardiac output was unchanged despite decreased
RV contractile function. The RV diastolic stiffness constant was not affected $\left(0.180 .1+\right.$ to $\left.0.210 .07 \mathrm{ml}^{-1}, \mathrm{p}=\mathrm{NS}\right)$. In the banding group, LV end-systolic pressure was decreased from 6816 to $2821 \mathrm{mmHg}(p<0.01)$. As in the control group, both endsystolic and end-diastolic volumes were increased $(p<0.01)$. In the banding group, acute LV unloading also depressed RV contractile function but significantly increased cardiac output. The RV diastolic stiffness constant was decreased from 0.300 .09 to $0.200 .07 \mathrm{ml}^{-1}(\mathrm{p}<0.05)$. In both groups, mean aortic pressure was maintained during acute LV unloading.

Conclusions: Both in normal as well as in hearts subjected to chronic RV pressure overload, acute LV unloading results in decreased RV contractile performance. In normal hearts, RV hemodynamic function is maintained during LV unloading. In contrast, in the pressure overloaded RV, pump function is improved during acute reductions in LV pressure, most likely as a result of improved $\mathrm{RV}$ diastolic compliance.

\section{P118}

Preoperative prediction of postoperative complications following paediatric cardiac surgery by immunophenotyping and humoral analysis

A. Trnok, J. Bocsi, P. Osmancik, J. Hambsch, G.K. Valet, P. Schmeider Pediatric Cardiology, Cardiac Center, University of Leipzig, Leipzig, $D$

Children who underwent cardiothoracic surgery frequently suffer from immediate postoperative complications (mild oedema, pericardial and pleural effusions, capillary leak syndrome, multiple organ failure) that needs extended intensive care and thereby substantially increases costs for therapy. Prediction of risk patients at the earliest possible time point could facilitate prophylactics. In the last years the immune response of $>100$ children who underwent cardiothoracic surgery was analysed. It was observed that patients who developed postoperative effusions and oedema (POEE) exhibited altered immunophenotype, antigen expression and serum concentration of various analytes already one day before surgery. Based on these findings a predisposition of risk patients that may allow their preoperative identification by data pattern analysis (SPSS, CLASSIF1) was hypothised. Three different classifiers were constructed and validated: 1 . Clinical data classifier (based on $\sim 40$ clinical and surgical data), 2. Serological classifier (cytokine and adhesion molecule serum levels, routine clinical laboratory data, $>80$ parameters) and 3 . Antigen expression classifier (cell count, adhesion molecule and activation marker expression on granulocytes and monocytes; 122 parameters). Although expectedly children with POEE underwent more complex surgical procedures Classifier 1 provided only poor discrimination (ARI (average recognition index) $<60 \%$ ). Classifiers 2. and 3. both yielded ARIs of $>85 \%$. A test set of patients unknown to the classifiers gave similar results. The number of parameters needed for discrimination was reduced to nine (Classifier 2) or seven (Classifier 3). These parameters indicated atopic/allergic phenotype of risk patients supporting the predisposition hypothesis. Individual risk assessment based on data pattern analysis could in near future aid clinical decision making. For clinical use these classifiers have to be tested and optimised on a larger scale of patients. The final classifiers can be adapted by other hospitals after calibration with a limited number of patients. Because the serological classifier is based on techniques available in most clinical laboratories it could be more easily adapted. However, the antigen expression classifier relies on a lower amount of sample material making it an ideal tool for neonates and infants. (Supported by Schsisches Ministerium fr Wissenschaft und Kultur, and Deutsche Stiftung fr Herzforschung). 


\section{P119}

Allo-/Autoreactive antibodies in patients with protein losing enteropathy (PLE) who underwent Fontan surgery D. Lenz, J. Hambsch, U. Sauer, J. Richter, J. Hess, P. Schneider, A. Trnok

Pediatric Cardiology, Cardiac Center, University of Leipzig, Leipzig, D

PLE is a feared late complication occurring $5-10$ yrs after Glenn/Fontan type of cardiac surgery with $5-15 \%$ of the patients exhibiting a substantial decrease of serum protein by increased secretion of protein into the gut. Mortality among patients with a manifest PLE is $>60 \%$. PLE aetiology is yet completely unknown. Two single case studies indicate that PLE is accompanied by the dramatic loss of T-helper lymphocytes. This loss most possibly causes reduced immunoglobuline (Ig) levels and recently reported opportunistic infections. The reason for the massive T-helper cell loss is until now unknown. In our study we tried to find a rational for this selective cell loss.

In a follow-up study we analysed 20 patients who underwent Fontan surgery over a period of up to five years by flow cytometry (FCM) and serology. One of these patients developed PLE nine months after surgery. Its immune alterations during PLE were compared to that of seven patients with a manifest PLE, PLEfree Fontan patients and healthy controls.

The immune alterations after PLE were comparable for all affected children. It included the dramatic selective loss of $>80 \%$ of the circulating T-helper (CD4 and a,b T-cell receptor positive) cells and the $>70 \%$ decrease of serum Ig levels (both $p<0.05$ ). We tested the serum of the patients for autoantibodies binding to cells and tissues by novel quantitative assays with FCM and Laser Scanning Cytometry. In total $87.5 \%(7 / 8)$ of the PLE patients had autoantibodies that were of heterogeneous specificity: against T-helper cells (3), against myocardial structures (3), against -cells of the pancreas (2). One patient displayed antinuclear antibodies. Serum of PLE free Fontan patients and of healthy controls was negative.

This is the first study showing that auto- and/or alloreactive antibodies are present in the majority of children with PLE. Because of these results we hypothise that autoimmunity is at least associated with if not participates in the aetiology of PLE and may contribute to selective T-cell depletion. Thus, autorectivity should be taken into account as one possible cause of PLE. (Support: Maximilian research award 1997, research grants of Herzkind e.V.).

\section{P120}

Blood-flow dependent concentrations of PTHrP in the pulmonary arterial system in children before and after closure of an ASD

R. Zimmermann, H. Degenhardt, J. Kreuder, I. Michel-Behnke, D. Schranz, H.M. Piper, K.D. Schlter

Paediatric Heart Center, University of Giessen, Germany, Giessen, D

Parathyroid-hormone-related peptide (PTHrP) is a paracrine factor expressed throughout the body. One of the multiple characterised physiological functions of this poly-hormone is vasodilation. In vitro we have been able to show that the endothelial release of PTHrP is blood flow dependent via a mechanosensitive mechanism.

Method: The concentrations of PTHrP were analysed in 12 children (age 5-7) undergoing interventional occlusion of their atrial septal defect (ASD). Blood samples were taken from the left atrium (LA), the left pulmonary artery (LPA) and a femoral artery before and 20 minutes after placing the occluder. PTHrPconcentrations were determined by a quantitative immunoblot and compared by Mann-Whitney-U-test.
Results: Before ASD-occlusion Qp/Qs was 2,1 (1,25, Rp/Rs 0,046 0,025 . The ratio of $\mathrm{PTHrP}$-concentration $\mathrm{LPA} /$ femoral artery and LPA/LA was $1,30,22(\mathrm{p}=0,001)$ and $1,240,26(\mathrm{p}=0,012)$ respectively. After occlusion (Qp/Qs 1,0 in all children) the pulmonary to systemic PTHrP-ratio (LPA/femoral artery) decreased to $1,140,13(\mathrm{p}=0,005)$. $\mathrm{Rp} / \mathrm{Rs}$ increased to $0.0870,04$.

Conclusions: An increased volume-load of the right heart leads to an increased PTHrP-concentration in the pulmonary arterial system. The increased $\mathrm{Rp} / \mathrm{Rs}$ after occlusion suggests a functional relevance of PTHrP. However, it remains unclear, where exactly PTHrP is released and where it is metabolised. Further studies to answer these questions and to examine the effect of an increased in vivo pressure load (pulmonary hypertension) on the PTHrPmetabolism are under way.

\section{P121}

Increased levels of troponin $I$ in chronic right ventricular pressure overload

M. Cappelli-Bigazzi, G. Santoro, C. Battaglia, P. Sorrentino*, L. Romano*, R. Calabr

Division of Pediatric Cardiology, "Monaldi" Hospital, Naples, I

Cardiac troponin I is uniquely expressed in myocardial cells and its release in the systemic circulation reflects the amount of myocardial damage. Indeed, elevated circulating levels of troponin I have been found after myocardial ischemia, open-heart surgery as well as in left ventricular (LV) failure or LV chronic pressure overload. Since only few data have been published about troponin I release in right ventricular (RV) damage, we evaluated its reliability as a marker of severity in chronic RV pressure overload. Arterial troponin I levels were measured in 17 children ( 10 males, 7 females; age $41.7+14.7 \mathrm{mos}$ ) with RV pressure overload due to isolated pulmonary valve stenosis (transvalvular pressure gradient $47.0+$ $4.0 \mathrm{mmHg} ; \mathrm{RV} / \mathrm{LV}$ pressure ratio $0.83+0.07$ ) undergoing pulmonary valvuloplasty and in 11 age-matched control children ( 6 males, 5 females; age $65.5+9.1$ mos; $p=N S$ ) with normal $\mathrm{RV}$ pressure $(\mathrm{RV} / \mathrm{LV}$ pressure ratio $0.26+0.01)$, undergoing percutaneous closure of small patent ductus arteriosus (mean Qp/Qs $1.12+0.05)$. Patients with RV pressure overload showed higher circulating levels of troponin I (Bekmann Access chemiluminescent immunoassay) compared with controls $(0.046+0.08$ vs $0.028+0.004 \mathrm{ng} / \mathrm{ml}, \mathrm{p}<0.05)$ and these values closely paralleled the severity of the RV pressure overload, as expressed by $\mathrm{RV} / \mathrm{LV}$ pressure ratio $(\mathrm{r} 2=0,80)$.

In conclusion, circulating troponin I significantly increases in patients with chronic RV overload, being potentially used as a reliable marker of this condition. Furthermore, due to its close relationship with the RV/LV pressure ratio, troponin I might be used as a sensitive index to guide the indication for RV pressure overload relief.

\section{P122}

Myocardial gene expression analysis using real-time RT-PCR in paediatric patients - methodological considerations and validation of potential reference genes D. Rawer, H. Akintrk*, A. Borkhardt\#, D. Schranz, J. Kreuder Department of Paediatric Cardiology, Justus Liebig Liniversit, Giessen, D

Real-time, fluorescent RT-PCR is a highly sensitive method to quantify gene expression. The choice of a reference gene and the quality of pre-PCR protocols like reverse transcription may markedly influence the validity of this method. Therefore, the different steps of real-time RT-PCR and potential reference genes 
'ere systematically evaluated for their applicability on myocardial ene expression analysis in hearts with congenital malformations. laterial: Right ventricular myocardium was taken from 2 patients with tetralogy of Fallot (age 4 months -18 years) durig primary corrective operation $(\mathrm{n}=18)$ or secondary operation $1=4)$.

lethods: The following steps of real-time PCR were separately camined: 1. RNA extraction using TRIzol(TM) and incubation ith DNAfree(TM) (Ambion) $\rightarrow$ quantification by spectrophometry, 2. real-time PCR for DNA specific single-copy c-Myc $\rightarrow$ measurement of contaminating genomic DNA, 3. reverse tranription $\rightarrow$ quantification of cDNA using OliGreen (TM) Uolecular Probes), a fluorescent nucleic acid stain, after preicubation with RNAse $\rightarrow$ comparison of RNA vs cDNA conentrations (efficiency of RT); 4. real-time PCR using ABI Prism 700 Taqman(TM) $\rightarrow$ determination of copy numbers by stanard curves (ranging between $10^{\wedge} 1$ and $10^{\wedge} 8$ copies per well) of JNA plasmids (Invitrogen). Several genes were analyzed garding their validity as reference genes: abelson $(\mathrm{ABL}), 2-$ icroglobulin (B2M), glyceraldehyd-P-dehydrogenase (GAPDH), spoxanthine-phosphoribosyl-transferase (HPRT), phorphobilinoin deaminase (PBGD), -glucuronidase (GUS), calsequestrin 2 ZASQ2), 185 ribosomal RNA (18S) and large ribosomal protein 0 (RPLPO), the latter two being intronless and thus not mRNAsecific.

.esults: The efficiency of RT varied from $23 \%$ to $104 \%$, emphazing the importance of cDNA measurement for reference gene alidation. PCR proved to have high efficiency (ranging from $3 \%$ for CASQ2 to $105 \%$ for $18 \mathrm{~S}$ ) and low intra-assay variability $1.9 \%$ for ABL to $11.2 \%$ for $18 \mathrm{~S}$ ). Sample-to-sample variation of opy numbers related to cDNA content was lowest for HPRT ;2\%), ABL (35\%) and PBGD (36\%) and highest for GAPDH , $0 \%$ ).

onclusion: For quantitative real-time RT-PCR of myocardial ssue in children and adolescents PBGD, HPRT and ABL appear , be the most suitable reference genes. The simultaneous use of veral reference genes may be useful

123

nesthesia and surgical trauma, but not ardiopulmonary bypass, are responsible for decreased eutrophil activity during pediatric cardiac surgery Hambsch, A.Tarnok, P.Osmancik, P.Schneider

Ieart Center Leipzig, University Leipzig, Leipzig, D

bjective: Pediatric cardiovascular surgery may lead to postoperive complications as postcardiotomy or capillary leak syndrome. he activation of neutrophils by the CPB is still held responsible ir these complications. However, present data do not show evience if surface expression and serum concentration changes of thesion molecules are $\mathrm{CPB}$ or surgery specific. Furthermore, udies were set in a short time range (from surgery up to 1-2 days ter surgery) thus do not include baseline values of the unperurbed immune status of the patients.

1ethods: 47 surgeries with CPB (aged $1-17$ yrs., CPB group) and 7 surgeries without CPB (aged 1-15yrs., control) were studied. lood was sampled $24 \mathrm{~h}$ preoperatively before any in-hospital redication, after anesthesia onset, after connection to $\mathrm{CPB}$, at sperfusion, $4 \mathrm{~h}, 1 \mathrm{~d}, 2 \mathrm{~d}$ after surgery, at discharge and months ostoperatively at the ambulance control. Neutrophil antigen xpression and serum concentration of soluble adhesion moleules were analyzed by flow cytometry and ELISA, respectively. esults: Anesthesia and surgery induced significant and transient ecrease of LFA-1 (CD11a/CD18), Mac-1 (CD11b/CD18) and
CD $5+$ surface expression as well as of ICAM-1 and L-selectin serum level below baseline (all $p<0.001$ ). Except L-selectin the decrease of all measured parameters was independent of the use of $\mathrm{CPB}$. In the $\mathrm{CPB}$ group L-selectin decrease was more pronounced $(p=0.006)$. With CPB CD62L (L-selectin) surface expression increased over baseline and control $(p=0.002)$. Antigen expression did not depend on $\mathrm{CPB}$ duration. Preoperative baseline values were reached $>2$ days to months postoperatively.

Conclusion: Cardiovascular surgery leads to suppression of neutrophil adhesiveness that is further reduced by CPB (see CD62L). Activation of neutrophils caused selectively by CPB can not be exclusively responsible for post-operative complications specific for $\mathrm{CPB}$ supported cardiovascular surgery. Baseline values are reached days or months postoperatively emphasizing the need to extend the time frame analyzed.

\section{P124}

Heart development in congenital diaphragmatic hernia

J. Correia-Pinto, M.J. Baptista, C. Pedrosa, L. Pina-Cabral,

Estvo-Costa, J.C. Areias, A. Leite-Moreira

Department of Physiology, Faculty of Medicine, Porto, $P$

Objective: Cardiac indices to assess left ventricular underdevelopment have been proposed to be predictive of lung hypoplasia and outcome in foetuses and newborns with congenital diaphragmatic hernia $(\mathrm{CDH})$. Preceding studies on cardiac effects in the nitrofeninduced rat model of $\mathrm{CDH}$ have been unclear. The purpose of this study was to investigate whether nitrofen exposure, mechanical compression from $\mathrm{CDH}$, or both, are determinants of cardiac growth abnormalities in the nitrofen-induced $\mathrm{CDH}$ rat model.

Methods: $\mathrm{CDH}$ was induced in pregnant Wistar rats by administration of nitrofen on E9.5. At selected gestational ages (E18, E20 and E22), the lungs and heart were harvested, weighed, and analyzed for DNA and protein contents. Two experimental groups: nitrofen without $\mathrm{CDH}$ (nitrofen), and nitrofen with $\mathrm{CDH}$ $(\mathrm{CDH})$, were compared with normal controls

Results: At E18, both nitrofen-exposed groups presented similar and significant left lung (LL) hypoplasia. As gestation progressed (E20 and E22), nitrofen-induced LL hypoplasia decreased, whereas the CDH-induced LL hypoplasia was exacerbated relative to normal controls. In contrast, at E18 and E20, heart-to-body weight ratios as well as cardiac DNA and protein contents were significantly reduced in all animals exposed to nitrofen, with no significant differences observed between nitrofen and $\mathrm{CDH}$ groups. As gestation progressed, the difference berween cardiac parameters in nitrofen exposed and normal control rats diminished and at E22 no significant differences were recognized.

Conclusion: We conclude that nitrofen exposure rather than mechanical compression from $\mathrm{CDH}$ is the only determinant of foetal heart underdevelopment and that cardiac growth abnormalities are transient and do not correlate with the degree of pulmonary hypoplasia.
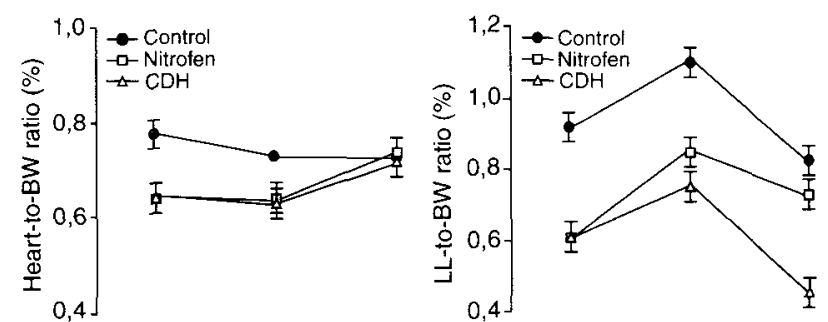


\section{P125}

Myocardial DNA and protein changes in a rat model mimicking cyanotic heart disease: effect of chronic hypoxia on myocardial growth patterns

N. Azar, G. Dbaibo, M. Nasser, M. El Sabban, H. Bitar,

S. Mroueh, M. Obeid M, F.F. Bitar

American University of Beirut, Beinut, $L E B$

Background: Myocardial growth during fetal life is accomplished by proliferation of myocytes. Shortly after birth, normal myocytes largely lose the capability to replicate.

Aim: The aim of this study is to assess the effect of chronic hypoxia on myocardial growth patterns in an animal model of chronic hypoxia, mimicking cyanotic heart disease.

Methods: Sprague-Dawley rats were placed in a normobaric hypoxic environment at birth and oxygen levels were maintained at $10 \%$ in an air-tight Plexiglass chamber. Controls remained in room air. Animals were sacrificed and the hearts were harvested at 1,4 and 8 weeks, respectively.

Results: Significant polycythemia developed in the hypoxic rats. There was significant increase in indexed RV and LV masses as compared to controls. Myocardial DNA concentrations were significantly increased in both hypoxic ventricles. For the RV, the increase in DNA content was $135 \%, 132 \%$ and $112 \%$ that of (C) at 1,4 and 8 weeks, respectively. $\mathrm{RV}$ and $\mathrm{LV}$ myocardial protein/DNA ratios were lower in the 1 and 4 week hypoxic rats, and significantly higher in the hypoxic 8 week old rats. Hydroxyproline concentrations and dry/wet weight ratios were not significantly different in $\mathrm{LV}$ and $\mathrm{RV}$ in both $(\mathrm{H})$ and $(\mathrm{C})$ animals.

Conclusion: Cardiac mass increases in response to chronic hypoxia, more in the RV than LV. This increase in cardiac mass is mainly due to myocardial proliferation in the first 4 weeks of life. Although the three groups of hypoxic rats had significant elevation in DNA concentration compared to controls, there is a shift from proliferation to hypertrophy after week 4 of life. The age of the myocyte appears to be the most important factor in triggering proliferation in our hypoxic animal model, rather than the duration or persistance of hypoxia.

\section{P126}

Versatile computer model of the heart and circulation with ability to evaluate treatment strategies in congenital heart diseases

T. Delhaas, M. Janssen, W. Jennekens, M. Liedenbatm, R. Pijnenburg, R. Vullings, T. Arts

Dept. of Pediatrics, Lniversity' Hospital Maastricht, Mastricht, $\ L$

We developed a lumped-parameter computational model of the heart and circulation to study the effects of valve size, valve insufficiency, septal defect size, ductus arteriosus or systemic-topulmonary shunt diameter and resistance, systemic and pulmonary resistance, preload, and heart rate on cardiovascular dynamics. The model is composed of modules, representing e.g. a cardiac chamber, a vessel segment, a valve or a septal defect. Differences are reflected by defining appropriate parameter values for each module. Active and passive sarcomere properties were used to calculate myocardial fiber stress and strain for each cardiac chamber (left and right atrium/ventricle). Cavity pressure and volume were derived from fiber stress and strain using the following relations: fiber strain $=1 / 3$ Delta $\ln (1+3$ (cavity volume $/$ wall volume $))$ and fiber stress $=$ cavity pressure $(1+3$ (cavity volume/wall volume $))$. Left and right ventricular interaction was simulated. Atrial inlets, atrioventricular and ventriculoarterial valves, as well as the septal defects, the shunt or the ductus were simulated as non-line: resistances. Flow could be regulated quantitatively, and was eithe uni- or bidirectional. Pulmonary and systemic circulations wer composed of non-linear compliances and resistances. Simulation: using morphometric parameter values for an adult $(75 \mathrm{~kg} .1 .75 \mathrm{~m}$ body surface area) as extracted from literature, showed tha 1) physiologically reliable left and right ventricular cavity volum and pressure can be calculated using sarcomere dynamics and a sim ulated left-right ventricular interaction; 2) the model reveals physi ologically reliable values for cardiac output, cavitary pressures an volumes, and arterial and venous pressures under a wide range c heart frequency and pre- and afterload. We conclude that the modt can be tuned to individual patients allowing routinely used indict to be computed, and that it is a promising tool in evaluating opera tive and interventional strategies in congenital heart disease.

P127 (see Abstract 21)

A heterozygous SCN5A point mutation in the domain I-II linker leading to LQT syndrome with $2: 1 \mathrm{AV}$ block, ventricular hypertrophy, contraction \& relaxation abnormalities

. G. Gentillig, I. Witters, B. EYskens, L. Mortens, T. Rossenbacker, H. Heidbchel, X. Wehrens, R. Jongbloed, R. Kass

Kindercardiologie, Lewen, $B$

\section{P128}

Fetal cardiac arrhythmias: Etiology, incidence of structural heart defects, treatment and long term outcom E. Marianne, B. Talvikki, A. Sture

Helsinki University Hospital, Helsinki, SF

The objectives of this follow up study of 292 fetuses with differ ent cardiac arrhythmias were to establish the incidences of struc tural heart defects and fetal compromise, to demonstrate the effec of anti-arrhythmic medication and to evaluate perinatal mortalit and morbidity and long term outcome.

The arrhythmias were differentiated into atrial extrasystoles (AEs $\mathrm{N}=200$ ), atrial tachycardias (AT, $\mathrm{N}=35$ ), atrioventricular bloc (AVB, $\mathrm{N}=36$ ), sinus bradycardia ( $\mathrm{SB}, \mathrm{N}=14$ ) and ventricula extrasystoles (VES, $N=7$ ). The incidence of heart defects $c$ tumors was $13 \%$ in the whole study population; $9 \%$ in AES, $6 \% \mathrm{i}$ AT, $17 \%$ in AVB, $43 \%$ in SB and $71 \%$ in VES. In utero cardiac fail ure (hydrops or pericardial effusion) was noticed in $12 \%, 1.5 \% \mathrm{i}$ AES, $48 \%$ in AT, $33 \%$ in AVB, $14 \%$ in SB and $14 \%$ in VES. Amon fetuses with AES, $1 \%$ developed supraventricular tachycardi (SVT) after birth. Among fetuses with AT SVT was present $\mathrm{i}$ $60 \%$, atrial flutter (AF) in $35 \%$ and intermittent SVT in $5 \%$ During antiarrhythmic therapy, sinus rhythm was achieved in $92^{\circ}$ of non-hydropic and in $63 \%$ of hydropic fetuses. Hydropic fetuse had higher mortality and risk for neurological disorder compare to non-hydropic fetuses; $38 \%$ vs $3.7 \%$ and $40 \%$ vs $12 \%$, respec tively. Among AVB fetuses without heart defect the total surviv: was $83 \%$. Prenatally developed cardiac failure was associated wit mortality of $50 \%$. In AVB fetuses with heart defect prognosis wa poor; $50 \%$ survived. SB and VES were associated with survive rates of $75 \%$ and $67 \%$. SB fetuses had long QT syndrome in $21 \%$ In the follow up of the whole study population of a median of years, $93 \%$ were alive and $4 \%$ had neurological disorder.

In conclusion, all other fetal arrhythmias but AES were associate with risk for cardiac anomalies or fetal compromise. In case of com promise the prognosis was poor and indicates effective perinat: treatment. After the newborn period the prognosis is good: howeve the risk of neurological disorder must be taken into consideration. 
$? 129$

The significance of tricuspid valve regurgitation in etuses between 11 and 14 weeks gestation

'. C. Huggon, T. Ghi, N. Zosmer, L.D. Allan

King's College Hospital, London, $\mathrm{CK}$

Zeasons for an unexpectedly frequent finding of tricuspid valve egurgitation in high-risk fetuses undergoing early fetal echocarliography were investigated.

Fetuses between 11 and 14 weeks gestation were selected for tetailed echocardiography. Referral reasons included increased uuchal translucency (generally more than $4.0 \mathrm{~mm}$ ), a suspected carliac or extra-cardiac malformation or a family history of congenial heart disease. The fetus was imaged transabdominally and pulsed Joppler of the atrioventricular valves was recorded if possible subsequently, the fetal karyotype was ascertained by chorionic illous sampling.

Julsed Doppler recordings of the tricuspid valve were obtained in 262 fetuses and tricuspid regurgitation was detected in $70(27 \%)$ Of these $70,58(83 \%)$ proved to have karyotype anomalies whereas $\mathrm{n}$ the absence of tricuspid regurgitation, 68/192 (35\%) fetuses had caryotype anomalies. The chromosome defect most frequently ound to have tricuspid regurgitation was trisomy 21 but all types of saryotypic anomalies were seen in association. Structural heart defects were detected in $40 / 70$ with tricuspid regurgitation $(57 \%)$ and in 27/192 (14\%) without. Six fetuses with tricuspid regurgitaion had neither karyotype abnormality nor a heart defect on the nitial scan, but in one of these coarctation became evident later and inother pregnancy was terminated for progressive hydrops. Thus, only 4 of the 70 fetuses with detectable tricuspid regurgitation had i normal outcome.

A careful search for tricuspid regurgitation is an important aspect of he evaluation of the early fetus, as this is frequently a marker for shromosomal defects even in the absence of structural heart disease.

P130

Increased nuchal translucency and jugular lymphatic development in the trisomy 16 mouse

X.M.S. wan den Akker, M.C. Haak, D. G. Jackson, S. Webb,

A.C. Gittenberger-de Groot

Dept of Anat and Embr, Leiden Lniversity Medical Center, Leiden, $N L$

Nuchal Translucency (NT) is a widely used screening method for hromosomal abnormalities in the late first trimester of pregnancy. It is also associated with a wide variety of other abnormalities, among which heart malformations. This transient accumulation of fluid in the subcutaneous region of the neck of the fetus can be visualised and measured by ultrasound. The (patho)morphogenesis of increased NT is still unexplained. A plausible hypothesis is that NT is a subcutaneous accumulation of fluid due to a discrepancy between formation and drainage of lymphatic fluid. It has been described that lymphatic development starts with bilateral bud formation from the internal jugular vein (IJV). They fuse and form the jugular lymphatic sacs. Later in gestation, these sacs reconnect with the IJV. Subsequently, the sacs differentiate into lymph nodes. A disturbance in this process could lead to a non-functional or delayed functional lymphatic system and explain the increased interstitial accumulation of fluid.

We studied the development of the lymphatic system using embryonic wild type and trisomy 16 mice (the animal model for Down's syndrome). At several embryonic stages, serial sections were stained for LYVE-1 and VEGFR3, both markers for lymphatic endothelium. The lymphatic development in wild type and trisomy 16 mice was compared. In early embryonic stages lymphatics and veins showed overlapping characteristics. Studying later stages, in trisomy 16 mice the lymphatic sacs were larger, more branches were seen and patches of lymphatic endothelium seemed thickened. Furthermore, an opening of the jugular sac into the IJV was never cncountered in both wild type and trisomy 16 mice. Instead, a membrane consisting of two layers of endothelium between the jugular sac and the IJV was observed in both trisomy 16 and wild type mice on E13 and E14. Similar findings were seen in human trisony 18 and 21 fetuses (12-16 weeks) with increased NT.

In conclusion, it is obvious that the lymphatic development is abnormal in trisomy 16 mice, possibly due to an endothelial differentiation problem. These abnormalities could help to explain the mechanism of increased NT and heart malformations seen in human fetuses with chromosomal abnormalities.

\section{P131}

Does fetal ventricular diastolic function influence the mobility of the primary septum flap valve? P. Zielinsky, C. Firpo, A.M. Aramayo, L.H. Nicoloso, E. Gus, A. Picoli, F. Sattler, J.L. Manica, H. Vaz

Fetal Cardiology Unit - Institute of Cardiology of RS, Porto Alegre, RS, BR

Backyround: Mobility of the fetal septum primum (SP) is a normal diastolic phenomenon. The high-velocity flow from the ductus venosus crosses the foramen ovale and prompts the flap valve to stretch toward the left atrial (LA) cavity. We hypothetized that the degree of the diastolic flap valve excursion could be related to left ventricular (LV) filling function.

This study was designed to test the hypothesis that the diastolic linear displacement of the SP is decreased in the presence of fetal LV hypertrophy (potential impaired diastolic function) and increased during fetal respiration movements (enhanced LV compliance) Methods: Two models were utilized. In the first, we compared by fetal echocardiography the ratio between the linear displacement of the flap valve and the LA diameter ("excursion index" [EI]) in 10 fetuses of diabetic mothers (FDM) with septal hypertrophy (SH) after 32 weeks of gestation, 8 FDM with normal septal thickness and 8 normal fetuses of mothers without diabetes (controls). In the second model, 28 fetuses were studied in apnea and during respiratory movements, comparing the EI of the flap valve in these two behavioral states. Statistical analysis utilized ANOVA and t-test. Results: Comparision of the three groups of the first study showed that, in FDM with $\mathrm{SH}$, the EI was 0.360 .09 , in FDM without SH it was $0.510 .09(\mathrm{p}=0.001)$ and in the control fetuses it was $0.490 .12(p=0.003)$. In the second study, fetuses in apnea had a mean EI of the flap valve of 0.390 .05 and during respiration it increased to $0.570 .07(\mathrm{p}<0.001)$

Conclusions: Mobility of the septum primum is reduced in left ventricular hypertrophy (decreased ventricular compliance) and is increased during fetal respiration (potential increase in ventricular compliance). We suggest that the measurement of the excursion index of the flap valve of the primary septum may contribute to the assessment of fetal diastolic function.

\section{P132}

Identification of impending fetal cerebral hypoxia during placental circulatory insufficiency

M.J. Raboisson, J. C. Fouron, J. Gosselin, C. Fouron, F. Proulx, S. Gamache

Fetal Cardiology Unit, Ste Justine Hospital, Montréal, CDN

Ojective: To assess the value of a new fetal Doppler aortic isthmus index, as marker of cerebral hypoxia during placental circulatory insufficiency (PCI) 
Background: A previous study (Am J Obstet \& Gynecol 2001;184:630-6) has shown that fetuses with PCI and predominant reverse flow in the aortic isthmus presented post-natal abnormal neuro-developmental outcome (NDO), possibly related to contamination of cerebral blood by very low $\mathrm{O} 2$ saturated red cells ejected by the right ventricle. However, there is no available mean of assessing the precise time when failure of compensatory mechanisms against cerebral hypoxia is about to occur.

Methodolgy: An index expressing the continuous changes in flow patterns through the fetal aortic isthmus has been designed: isthmic flow index $(\mathrm{IFI})=($ systolic + diastolic $) /$ systolic flow velocity integrals. The IFI values of 30 singleton pregnancies with abnormal umbilical artery Doppler were retrospectively divided in four groups: group I: IFI $>1$ (antegrade diastolic flow) $n=12$; group II: $0.5<$ IFI $<1$ (mild retrograde diastolic flow and antegrade systolic flow) $\mathrm{n}=9$; group III: $0<$ IFI $<0.5$ (moderate retrograde diastolic flow and systolic flow predominantly antegrade) $n=5$; group IV: IFI $<0$ (predominant retrograde flow) $\mathrm{n}=4$. The last IFI value before delivery was correlated with post-natal NDO. Results: Mean gestational age at delivery was 33.32 .4 weeks and mean birth weight $1568 \mathrm{~g} .4 .11$. The last NDO was assessed at an average of 4.3 years 1 . The NDO was abnormal in $5 / 12$ children of group $1,7 / 9$ of group II and in all children of groups III $(5 / 5)$ and IV (4/4). Incidence of abnormal NDO was significantly higher in groups III and IV compared to groups I and II $(p<0.01)$.

Conclusion: An IFI between 0.5 and 1 (group II) suggests impending fetal cerebral hypoxia.

\section{P133}

Fetal supraventricular tachyarrhythmias: a continuing medical challenge?

A. Benatar, G. Rondia, J. Leuris, H. Dessy, T. Decraene, K. Khaldi, P. Viart

Paediatric Cardiology, AZ VZB, Brussels, $B$

Fetal supraventricular tachyarrhythmias (SVT) may lead to hydrops fetalis and cause fetal death. Materno-fetal treatment strategies remain divergent and controversial. We evaluated our 18 year experience with fetal SVT for efficacy and outcome using different treatment strategies.

Methods: We diagnosed 36 fetuses with SVT, $75 \%$ in the last 10 years. Fetuses were divided into 2 groups: group 1 nonpharmacologically treated and group 2, pharmacologically treated. Results: Group 1: consists of 11 fetuses, gestational age 34-40 weeks, median 36 weeks, 4 presenting with hydrops. All the latter underwent expeditious delivery (by caesarian section). Group 2: consists of 25 fetuses, gestational age 17 to 38 weeks. median 30 weeks. Ten fetuses in this group were hydropic at presentation. Arrhythmia mechanism: AV re-entry tachycardia (AVRT) in 26, atrial flutter in 8, and atrial ectopic tachycardia (AET) in 2.

Maternal antiarrhythmic treatment: For the 10 hydropic fetuses: digoxin alone in 2 , digoxin + flecainide in 6 , amiodarone alone in 1 , and $>$ three drugs in 1 . The latter died at 23 weeks gestation during direct fetal umbilical treatment. For the 15 nonhydropic fetuses: digoxin alone in 10, digoxin + flecainide in 2 . digoxin + verapanil in 1, digoxin, verapamil and beta-blocker in 2. In 18 of 25 fetuses conversion to sinus rhythm was obtained, slowing of tachycardia rate in 1 , failure in 5 and death in 1 (AET). Conversion to sinus rhythm was achieved most rapidly when using digoxin and/or flecainide and have become our first-line drugs. No maternal mortality nor appreciable morbidity has been observed with these drugs in this small series

Conclusion: Flecainide with digoxin is efficacious and rapid in regaining sinus rhythm in haemodynamically compromised fetuses with AVRT SVT and structurally normal hearts who ar too premature to be delivered. The risk-benefit ratio, thus fa appears to be favorable.

\section{P134}

Simultaneous pulsed wave Doppler recording of pulmonary artery and vein in the fetus $-A$ tool to diagnose fetal arrhythmias

J.S. Carvalho, F. Prefuno, V. Ciardelli, E.A. Shinebourne

Royal Brompton Hospital, London, UK

Background to Objective: Fetal arrhythmias are conventionall assessed by $\mathrm{M}$-mode echocardiography (M-mode). This tech nique relies on image quality, is angle dependent and can be time consuming. We therefore, evaluated the potential of pulsed wav Doppler (PWI) in pulmonary vessels as an alternative diagnosti tool.

Methods and Patients: From July 1999, we have routinely use simultaneous PWD recordings of pulmonary artery (PA) an vein (PV) to assess prenatal arrhythmias. Until October 200. 45 patients (11-38 weeks) with arrhythmias were identifies Placement of sample volume was guided by colour flow mappin (4-chamber section) during fetal apnoea, using low velocity an low filter settings at $100 \mathrm{~mm} / \mathrm{s}$.

Results: Simultaneous recordings were obtained in $<5 \mathrm{~min}$ in $\mathrm{mo}$ cases and usually within $1-3 \mathrm{~min}$. It was not attempted in one fett $(11+5$ weeks) and in 6 , data was not available for analysis. In 4 c the remaining 38 fetuses, diagnosis was based on M-mode (atri ectopics) and in 34, PWD pattern was diagnostic (89.5\%). In 20 these, $\mathrm{M}$-mode was available and compared with Doppler. Ther were 25 cases of atrial ectopics (early 'A' wave), including blocke atrial bigeminism leading to ventricular bradycardia. In 3 , th ectopics were predominantly ventricular (early PA signal, compen satory pause). There were 5 supraventricular tachycardias. A showed summation of ' $S$ ' and ' $D$ ' waves and reversal of the ' $A$ ' wav on PV signal. Measurement of the ventriculo-atrial (VA) interv was possible in all. One had long VA interval (intermittent atri tachycardia) and 4 short VA intervals (reentrant mechanism). On fetus presented with atrioventricular (AV) block, ranging from fir to second degree. Interpretation of PV signal is not possible wit active fetal breathing movements and can take longer if the rhytht is very irregular. Audio signal was advantageous over $\mathrm{M}$-mode. Conclusions: Simultaneous PW Doppler recording of PA and P signals was easily obtained during fetal arrhythmias and allowe prompt diagnosis in most cases. It has become our first choice i the assessment of prenatal arrhythmias.

\section{P135}

Poor outcome of prenatally diagnosed Down syndrome fetuses with cardiac anomalies

I.M.E. Frolm-Mulder, M. Wessels, F. Los, P. Willems, M. Niermeyer, J. Wadimiroff

Sophia Children's Hospital/Erasmus MC, Rotterdam, $\mathrm{NL}$

Introduction: The outcome of fetuses who are diagnosed wit Down syndrome because of abnormal prenatal ultrasound finding that lead to the cytogenetic diagnosis of trisomy 21 is not we known.

Material and methods: To deternine the outcome of thes fetuses, 55 women were studied with sonographically suspecte fetal abnormalities, leading to the diagnosis trisony 21 in thei fetus. A detailed scan was performed to determine the nature $c$ the anomalies and possible associated malformations, and out come of the pregnancy was studied. 
Results: Congenital heart defects (CHD) were diagnosed pre- and postnatally in 29 out of these 55 fetuses (55\%), with complete or incomplete atrioventricular septal defects and ventricular septal defects being the most frequent anomalies. The most frequent noncardiac findings were a relative short femur (45\%) and smallfor-gestational age (SGA) (27\%). Termination of pregnancy was carried out in 25 of 55 pregnancies $(45 \%)$, the other 30 pregnancies, mostly beyond 24 weeks of gestation, were continued. Ten of these ended with intrauterine death at a mean gestational age of 35 weeks, of whom 5 were diagnosed with CHD, all presenting with congestive heart failure, and 7 were diagnosed with SGA. Of the 19 cases diagnosed prenatally with $\mathrm{CHD}$ in which the pregnancy was continued, $13(68 \%)$ died in-utero or in the first year of life. In the presence of combined CHD and SGA, 6 out of $7(86 \%)$ died inutero or in the first year after birth, as opposed to only 1 out of 6 infants $(16 \%)$ without $\mathrm{CHD}$ and with normal prenatal growth. Combining intrauterine death and death in the first year indicated that the overall survival rate in fetuses diagnosed prenatally with Down syndrome because of abnormal ultrasound findings was only $40 \%$

Conclusion: This study indicates that in-utero diagnosis of Down syndrome has a poor outcome when they present with CHD and/or SGA. This is important in the counseling of the parents.

\section{P136}

Closing of ductus arteriosus in fetal life analysis of 15 cases

K. Janiak, M. Respondek-Liberska

Institute "Polish Mother's Memorial Hospital", Lodz, PL

Objective: The analysis of clinical course of pregnancies complicated by prenatal closing of ductus arteriosus confirmed by fetal echocardiography at the referral center.

Material and methods: Among 4976 fetuses between January 1994 and September 2001. in 15 cases $(0,3 \%)$ prenatal closing of ductus arteriosus was diagnosed during fetal echocardiography (PI of DA was $<1.9$ ).

Results: Mean gestational age at the time of diagnosis was 33 wks $(30-39 \mathrm{wks})$. In 10 cases $(66 \%)$ maternal pharmacotherapy (Indomethacin, Betamethazon, Dexamethazon) was considered as a cause of the fetal echocardiographic diagnosis, and idiopathic closing was considered in 5 cases (34\%). In addition to abnormal ductal PI other echocardiographic anomalies were present, such as: myocardial hypertrophy, tricuspid regurgitation, abnormal foramen ovale flow, pulmonary regurgitation, disproportion, pericardial effusion. Two newborns presented also hydrops fetalis and died after delivery. There were 13 survivors, who had been delivered mean 3 weeks after the prenatal diagnosis and during the first day of postnatal life presented RV overload, however with no any symptoms at mean of 6 months of age.

Conclusions: Prenatal closing of ductus arteriosus may be due to maternal pharmacotherapy or idiopathic. In addition to ductus arteriosus abnormal flow usually other echocardiographic anomalies are present as well. Prenatal closing of ductus arteriosus may be only temporary and may have no adverse effect for survivor.

\section{P137}

Evaluation of myocardial performance in fetuses with univentricular hearts using the Doppler Tei-index

E. Lechner, G. Tulzer, R. Gitter, R. Mair, J. Hulta

Children's Heart Center Linz, Linz, A

In fetuses with univentricular hearts the remaining cardiac chamber has to take over the work of the missing ventricle. Therefore the whole cardiac output must be pumped by a single ventricular chamber leading to chronic volume overload. Nevertheless in the absence of significant atrioventricular valve regurgitation congestive heart failure rarely occurs. To assess the impact of chronic volume overload on myocardial performance in fetuses with univentricular hearts the Doppler Tei index was used. This index is independent of ventricular geometry and heart rate and can easily be obtained from a Doppler ventricular inflow and outflow trace. Tei index $=($ ICT + IRT $) / E T$, where ICT is isovolumetric contraction time; IRT: isovolumetric relaxation time and ET: ejection time. Published normal values show no difference between right and left ventricles and no change during gestation. A normal Tei index is considered to be less than 0,40 .

Tei index was measured retrospectively in 12 fetuses (Gestational age: $33 \pm 6$ weeks) with univentricular hearts (Hypoplastic left heart (HLHS): $n=7$; tricuspid atresia (TA): $n=4$; double inlet left ventricle: $n=1$ ). None of the fetuses had any other extra cardiac malformation, more than trivial $A V$ valve regurgitation, the venous Doppler measurements were normal and there were no detectable signs of heart failure in utero. All had birth weights within normal limits and underwent surgery within the first week of life. Tei index was $0,52 \pm 0,17$ (mean \pm std. dev). Only $25 \%$ $(3 / 12)$ had a normal Tei index of less than 0,40 (2 fetuses with HLHS, 1 with TA), 2/12 had an index of more than 0,80 ( 2 fetuses with HLHS). Although there was a tendency towards higher Tei indices in fetuses with single right ventricles versus single left ventricles, the difference was not significant.

Fetuses with univentricular hearts may have abnormal myocardial performance. The Tei index might be a sensitive and useful indicator to detect fetuses at risk for congestive heart failure.

\section{P138}

Effect of Idebenone on hypertrophic cardiomyopathy in Friedreich's ataxia

A. Fournier, J. Thrien, M. Vanasse, M. Mond, .M. Pandolfo

Hôpital Sainte-Justine, Montral, CDN

Freidreich ataxia is an autosomal recessive disease characterised by spinocerebellar degeneration and hypertrophic cardiomyopathy. It is caused by deficiency of frataxin, a protein involved in regulation of mitochondrial iron content. The effect of idebenone (a freeradical scavenger who can protect both membrane lipids and mitochondrial respiratory chain enzymes from iron-mediated injury) was assessed in 11 children and adolescent ( 8 girls, 3 boys). Baseline evaluations (mean age; $13.64 \pm 2.30$ years) were compared to evaluations after 6,9 and 12 months of idebenone $5 \mathrm{mg} / \mathrm{kg}$. No patient presented side effects and all were stable on the neurological standpoint. Cardiac evaluations included 12 lead electrocardiogram and echocardiogram where were measured muscle thickness, mass and indices of systolic and diastolic functions. On the electrocardiogram the only parameter that changed significantly was a decrease of $R$ wave in $V 6$ from $1.41 \pm 0.32 \mathrm{mV}$ to $1.25 \pm 0.39 \mathrm{mV}$, $p=0.02$. Thickness of the septum and the posterior wall in diastole and cardiac mass expressed in $\mathrm{Z}$ score decrease significantly throughout the study, respectively: $7.23 \pm 3.95$ to $5.10 \pm 3.56$, $\mathrm{p}<0005 ; 5.21 \pm 1.66$ to $4.13 \pm 2.06, \mathrm{p}<0005 ; 2.32 \pm 2.55$ to $1.40 \pm 2.42, p=0.001$. Shortening fraction and cardiac index did not change significantly. The only patient who necessitated treatment for obstructive cardiomyopathy presented a reduction of gradient despite the reduction of her beta-blockers dosage. In conclusion, idebenone seems very effective in decreasing left ventricular wall thickness and cardiac mass without influence on cardiac function in patient with Friedreich's ataxia. Only a longer follow-up will determine if the effects remain over time. 


\section{P139}

Spectrum of arterial stenoses caused by elastin point mutation ELN $526 \mathrm{~A}>\mathrm{T}$

A. Koch, G. Buheitel, M. Hofbeck, A. Rauch, B. Trusen, G. Hofner, M. Tassabehii, $H$. Singer

Univ.-Klinik fr Kinder und Jugendliche, Pd. Kardiologie, Erlangen, $D$

Supravalvular aortic stenosis (SVAS) is a frequent feature of Williams syndrome associated with a submicroscopic deletion involving the whole Elastin gene. In familial and sporadic cases of SVAS, gross deletions and widespread point mutations in the elastin gene have been described. We report the spectrum of arterial stenosis in two families with the same point mutation.

Family 1: At age 2.3 years, a long segment severe SVAS was diagnosed in a boy by cardiac catheterisation. The aortic arch distal to the innominate artery was of normal size. Moderate peripheral pulmonary artery stenoses were also seen. Surgical repair by patch aortoplasty was performed. At 7.1 years of age aortic angiography now revealed severe obstructions at the takeoff of the carotid and subclavian arteries and moderate stenosis of the left pulmonary artery. Elastin gene mutation ELN $526 \mathrm{~A}>\mathrm{T}$ was detected in the patient, his mother (history of surgical repaired SVAS), and his younger brother (up to now without cardiac evaluation). Fanily 2 : A 5.5-year-old girl had mild SVAS but significant peripheral stenosis and hypoplasia of both the right and left pulmonary artery (cardiac catheterization ?P $55 / 60 \mathrm{mmHg}$ ). Her brother underwent surgery because of severe SVAS at 5 months of age. Cardiac catheterisation at the age of 6.7 years showed no circunnscript narrowing but hypoplasia of the ascending aorta, and severe bilateral peripheral pulmonary stenosis. Elastin gene mutation ELN 526 A $>\mathrm{T}$ was confirmed in both.

Intrafamilial and interfamilial phenotypic variability in elastin point mutation ELN $526 \mathrm{~A}>\mathrm{T}$ is high. A single point mutation causes the complete spectrum of arterial stenoses known from Williams syndrome.

\section{P140}

\section{Epidemiology of paediatric heart disease in}

a developing country - an unchanged pattern

M. Sadiq, R. Bakht, F. Latif, I. Bashir, S.A. Sheikh

Punjab Institute Of Cardiology \& Childrens' Hospital, Lahore, Pak

Background: In the West, paediatric heart disease essentially means congenital heart disease (CHD). In our part of the world, however, rheumatic fever, rheumatic heart disease (RHD) and post-viral myocardial disease continues to be an important cause of hospital admission in children.

Objective: To assess the pattern, age distribution \& relative incidence of heart disease in all new paediatric patients (pts) aged 16 years \& less.

Design: A five-year (May 96-April 01) analysis of all children undergoing detailed echocardiography in a single tertiary referral center.

Patients \& methods: Data of all children was reviewed for type of lesion, age at presentation \& gender. Heart disease was divided into CHD \& acquired heart disease (AHD).

Results: Of 7400 new pts undergoing echocardiography, 6620 were found to have a cardiac lesion while 780 were normal \& excluded. Of these $6620 \mathrm{pts}, 4184(63.2 \%)$ had CHD while 2335 (35.3\%) had AHD. Of CHD, VSD was the most common lesion (32\% of CHD), followed by ASD (13.2\%) \& PDA (12.8\%). Majority were males ( $65 \%)$. Mean age of presentation was $5.8 \&$ 4.8 for acyanotic \& cyanotic heart defects. TOF was the commonest cyanotic lesion (16.06\%) with mean age of presentation
4.2 years. Only 586 pts $(14 \%)$ were under the age of one year \& $127 \mathrm{pts}(3 \%)$ were less than one month of age at presentation. Amongst AHD, 1670 pts $(71.5 \%)$ had RHD while 572 pts $(24.5 \%)$ had post viral myocardial disease. The mean age of presentation for myocarditis was 2.3 years and majority were clustered to the months of March, April and September, October. Amongst RHD, MR was the commonest lesion, $681 \mathrm{pts}(40.8 \%)$ followed by mixed lesion of MR \& AR, 382 pts (22\%) and mitral stenosis, 264 pts (15.8\%).

Conclusion: CHD forms the largest group of pts presenting to a tertiary referral center. Majority of children present late with a bias towards less severe heart defects. RHD continues to be an important cause of suffering among children in this part of the world. Myocardial disease especially myocarditis is also fairly common and has a seasonal variation indicating possible viral etiology:

\section{P141}

Congenital heart defects in monochorionic twin gestations

J. Dumontier, L. Fermont, J. Le Bidois, E. Villain, G. Agnoletti, D. Sidi, D. Bonnet

Hopital Necker Enfants Malades, Paris, $F$

Monochorionic twin gestations constitute a paradigm for the role of epigenetic factors and "chance" in the causes of congenital heart defects (CHD) as the monozygotic twins frequently have different hearts. Over a period of 6 years, we reviewed all twin sets issued of monochorionic twin gestations in which one or both twins had a proven CHD. There were 34 sets of twins (29 monochorionic diamniotic and 5 monoamniotic). Forty-four foetuses had a CHD: 14 tetralogies of Fallot, 10 right outflow tract obstructions (ROTO) with intact septum, 4 right isomerisms, 3 situs inversus. 4 coarctations with or without ventricular septal defect, 6 ventricular septal defects (VSD), 1 Taussig-Bing anomaly with interrupted aortic arch, 1 persistent ductus arteriosus, 1 hypoplastic left heart syndrome. Concordant CHD were observed in 6 pairs of twin (4 tetralogies of Fallot, 1 coarctation, I VSD with malalignment$17.6 \%$ ) and discordant defects in 28 pairs of twin (82.4\%). Among discordant pairs, we observed 10 ROTO of which 7 were in the recipient twin of a twin-twin transfusion syndrome, the other twin being normal in 8 cases and harboring a small muscular VSD. These ROTO ranged from mild pulmonary stenosis to pulmonary atresia. Discordance was also observed in 8 conotruncal defects (6 tetralogies of Fallot, 2 coarctations with ventricular septal defect), in 2 VSD, and 1 hypoplastic left heart syndrome; the other twin being normal. Finally, in 7 sets of twin, we observed situs anomalies with 4 right isomerisms (the other foetus being normal) in 4 monochorionic monoamniotic gestations and 3 situs inversus with structurally normal heart in 2 cases and double discordance in one case. Discordant heart structures is the most frequent situation in monochorionic gestations. Defects observed might be acquired such as ROTO in twin-twin transfusion syndrome. However, structural defects determined from the early gestation are frequent and also mostly discordant suggesting that in monochorionic twins, non genetic factors causes heart malformations.

\section{P142}

Epidemiology of congenital heart disease a multicentric national study in Croatia, 1995-2000

N. Rojnic Putarek, I. Rudan, H. Kniewald, M. Jelusic, D. Sudar, I. Malcic

Lniversity Hospital Rebro, Zagreb, CRO

Congenital heart diseases (CHD) comprise a group of aetiologically and phenotypically different conditions seen in $0,6-1 \%$ of 
live births. There is a tendency to classify CHD's according to Clark's pathogenic classification, which assumes that relatively few pathogenic mechanisms cause a wide spectrum of phenotypic forms of $\mathrm{CHD}$, which is in line with recently acquired knowledge from embryonic and genetic investigations. The aim of this study was to classify CHD in Croatian population according to Clark's classification, to calculate incidence rates of $\mathrm{CHD}$ in the total Croatian population and compare the results with other populations. We formed the Registry including all children with CHD treated in all 16 Departments of Paediatrics in Croatia, born between 1995 and 2000. Diagnosis of CHD was confirmed by echocardiography and in some cases by cathetherisation or operation. The data on total number of livebirths were obtained from the Croatian Institute for Public Health. All patients and liveborn children were divided by counties of the Republic of Croatia. Basic information and calculated incidence according to the year of occurrence is presented in Table 1.

Statistically significant difference was found between CHD incidence rates in several counties $(0,42-1,41 / 1.000$ live births). The incidence of all main pathogenic groups of CHD was constant in time, except for cell death defects where threefold increase in incidence of muscular ventricular septal defect (VSD) was noted $(0,5-1,5 / 1.000$ live births). This can be explained by better diagnostics of smaller muscular VSD. The most common diagnosis was perimembranous VSD (19.1\%), followed by atrial septal defect (ASD) type II $(14,4 \%)$, muscular VSD $(11,3 \%)$, pulmonary stenosis without VSD $(8,5 \%)$, patent arterial duct $(6,3 \%)$, subarterial VSD $(5,3 \%)$ and Tetralogy of Fallor $(3,9 \%)$. The incidence and diagnostic possibilities in our population are not significantly different from those presented in the literature. However, the differences in incidence of $\mathrm{CHD}$ between counties warrant the need for further investigation into the development and etiology of CHD.

\section{P143}

Morbidity and mortality in pediatric

heart-lung-transplantation - a single center experience

S. Reichel, L. Roemer, R. Kozlik-Feldmann, M. Buss, H. Netz,

S. Dbritz, B. Reichart

Department of Pediatric Cardiology, . Munich, D

Introduction: Heart-lung-transplantation (HLTX) is regarded as therapeutical option in persisting pulmonary hypertension. By now our center has exsperience in pediatric patients for over 9 years.

Patients and outcome: Since 199213 children (8 with primary pulmonary hypertension, 5 with secundary pulmonary hypertension) were heart-lung-transplanted. Mean age at transplantation was 9.5 years (range $2-15 \mathrm{y}$ ). Time after HLTX ranges from 1.5 to 88 months (mean 42.6 months). Immunosuppression is based largely upon Tacrolimus (TAC) and Mycophenolat Mofetil (MMF). 2 patients died, representing a mortality of $16 \%$. Cause for death were in both cases severe pulmonary infections. During surveillance of 520 patient months 7 patients suffered from acute graft rejection in 15 cases. Chronic rejection occured in 4 cases. Malignancys were seen in 2 patients as lymphoproliferative diesease. One case retreated after switch of immunosuppression. The second patient suffered from a malignant lymphoma, which had to be treted with chemotherapeutics. Sever infections, necessitating hospital admission, were seen in 34 cases during surveillance, thus corresponding to a rate of 0.05 severe infections per patient month. After HLTX patients were on average 19 days/year hospitalised, $54 \%$ of this because of infectious disease. 10 of 11 living patients are at present able ot attend regular school or kindergarten.
Conclusion: In pediatric HLTX by now exist exsperiences for over 5 years. It is a promising therapeutical option for children with persisting pulmonary hypertension. Patients can reach a very good quality of live after this procedure. Immunosuppression based on TAC and MMF leads to a good outcome in regards of rejections and infections.

\section{P144}

Influence of reparative surgery on the risk and prognosis of infective endocarditis in congenital heart diseases

S. Di Filippo, B. Semiond, F. Sassolas, M. Celard, A. Bozio

Paediatric Cardiology, Hopital Cardiologique Louis Pradel, Lyon, F

This aim of this retrospective study was to determine whether outcome and prognosis of infective endocarditis (IE) was influenced by previous corrective or palliative surgery in patients with congenital heart diseases (CHD)

From 1966 to 2000, 167 IEs occurred in 153 patients, aged 3.6 days to 78.5 years (y) (median $10 \mathrm{y}: 125$ children, 42 adults). Cases were divided in Groups: $\mathrm{A}=87$ unoperated, $\mathrm{B}=38$ palliated and $\mathrm{C}=42$ repaired. Ventricular septal defect (VSD) and tetralogy of Fallot (TOF) were the commonest underlying CHD in group $\mathrm{C}$, complex cyanotic CHD (CCC), TOF, complex acyanotic CHD (ACCC) and aortic valvulopathy in B, VSD, mitral valvulopathy, bicuspidia, $\mathrm{CCC}$ and $\mathrm{ACCC}$ in $\mathrm{A}(\mathrm{p}<0.0001)$. Recurrence was more frequent in $\mathrm{B}(\mathrm{P}=0.02)$. Cardiac surgery and dental causes were commoner in $\mathrm{C}$, dental and cutaneous causes in $\mathrm{B}$ and $\mathrm{A}(\mathrm{P}<0.0001)$. Unoperated patients (group A) had more clinical complications cardiac ( $38 \%$ vs $18 \%$ in $\mathrm{C}$ and $10.5 \%$ in $\mathrm{B}, \mathrm{p}=0.028)$ and extracardiac $(60 \%$ vs $23 \%$ in $\mathrm{C}$ and $27 \%$ in $\mathrm{B}, \mathrm{p}<0.0001)$ and higher incidence of embolias ( $45 \%$ vs $14 \%$ in $C$ and $18 \%$ in $B, p=0.0003)$. Early cardiac surgery was needed in $31 \%$ of the cases: $45 \%$ in group $A, 5 \%$ in $B$ and $26 \%$ in $C(p<0.0001)$. Freedom from cardiac surgery during follow-up (FU) was lower in group A $(20 \%$ at 10 y FU, vs $40 \%$ in B and $50 \%$ in $\mathrm{C}, \mathrm{p}=0.004)$. Thirty-four patients died $(21 \%), 1$ day to $19.5 \mathrm{y}$ after diagnosis (median $6 \mathrm{mths}$ ), from post-IE (48.5\%), cardiac nonIE $(39 \%)$ or unknown $(12 \%)$ causes. The incidence of death was higher in group B (35\% vs $19 \%$ in C and $15.3 \%$ in A, p $=0.043)$ Survival was $93 \%, 89 \%, 87 \%, 76 \%$ and $56 \%$, respectively at $1 \mathrm{mth}$, 6 mths, $1 \mathrm{y}, 10 \mathrm{y}$ and $25 \mathrm{y}$. Long-term survival did not differ between the 3 groups $(p=0.1)$

Our results show that reparative surgery does not prevent IE, but risk of cardiac complication, embolia and early surgical treatment is higher in unoperated patients; mortality is increased in palliated patients with underlying complex cardiopathies.

\section{P145}

Early surgery of infective endocarditis in congenital heart disease; outcome and prognosis S. Di Filippo, B.Semiond, F. Sassolas, M. Celard, A. Bozio Paediatric Cardiology, Hopital Cardiologique Lonis Pradel, Lyon, $F$

The aim of this retrospective study was to determine the prognostic significance of early surgical treatment for infective endocarditis (IE) in patients with congenital heart diseases (CHD).

From 1966 to 2000, 167 IE occurred in 153 pts, aged 3.6days to 78.5 years (y) (median $10 \mathrm{y}$ ); 125 were $<18 \mathrm{y}$ of age and 42 were adults. Underlying CHDs were: ventricular septal defect isolated (15\%: repaired $24 \%$, unoperated $76 \%)$ or not $(8 \%$ : repaired $60 \%)$, complex cardiopathy cyanotic (18\%: palliated $77 \%$ ) or acyanotic (7\%: palliated $33 \%$, non-operated $41 \%)$, Tetralogy of Fallot $(8 \%$ : repaired $50 \%$, palliated $36 \%$ ), aortic ( $9 \%$ including bicuspidia $2.4 \%$ and prothesis $4 \%$ ) or mitral ( $7 \%$ including MVP $2.4 \%$, non-operated 
$100 \%$ ) valvulopathies, and ductus arteriosus ( 2 non-operated), atrial septal defect + mitral anomaly $(3=2$ repaired, 1 non-operated), pulmonary stenosis (1 non-operated). Route was unknown (31\%), dental (25\%), post cardiac surgery (19\%), cutaneous ( $9 \%)$, ENT $(6 \%)$ or other. Blood cultures were negative in $14 \%$; streptococcus and staphylococcus were the commonest agents. Clinical cardiac complications occurred in $26 \%$, embolias in $31 \%$. Vegetations were the most frequent echographic lesion (42\%). Early surgery $(<3 \mathrm{~d}$ month after diagnosis) occurred in $31 \%$ of the cases and was indicated for haemodynamic instability, uncontrolled sepsis or multiple embolias. Risk factors for early surgical treatment were: underlying CHD (mitral and/or aortic valvulopathy, $\mathrm{p}=0.0008$ ), clinical cardiac failure $(p<0.0001)$, embolias $(p=0.0014)$, extracardiac complications due to embolia ( $\mathrm{p}=0.0026)$, site of IE (mitral and/or aortic, $\mathrm{p}<0.0001$ ), echographic lesions (vegetations, $\mathrm{p}<0.0001$ ). Age, period, time to diagnosis, bacterial agent were not significant factors. Early surgical treatment included mitral and/or aortic prosthesis $(50 \%)$, mitral repair (8\%), valvular homografts (8\%), Ross operation ( $8 \%$ ), interventricular patch (11\%), ductus ligation (2\%) and miscellaneous. Thirty-four patients died (21\%), 1 day to $19.5 \mathrm{y}$ after diagnosis (median $6 \mathrm{mths}$ ), from post-IE (48.5\%), cardiac nonIE (39\%) or unknown (12\%) causes. Survival was $93 \%, 89 \%, 87 \%$, $76 \%$ and $56 \%$, at $1 \mathrm{mth}, 6 \mathrm{mths}, 1 \mathrm{y}, 10 \mathrm{y}$ and $25 \mathrm{y}$. Early surgical treatment did not influence mortality nor survival.

Our results show that left heart cardiopathies and locations, clinical complications and echographic vegetations lead more often to early surgery without increase in mortality

\section{P146}

Global enddiastolic volume measured by transpulmonary indicator dilution adequately reflects changes of cardiac preload in children after corrective cardiac surgery Ch. Pauli, J. Wandel, L. Fakler, *.M. Hennig, J. Hess

Deutsches Herzzentrum München, Paediatric Cardiology, München, D

Management of intravascular blood volume is a major principle and of vital importance in critically ill patients. Our aim was to test whether the indexed Global Enddiastolic Volume (GEDVI) measured by Transpulmonary Indicator Dilution (TPID) reflects changes in cardiac preload in infants and children after corrective cardiac surgery.

The data of 105 consecutively examined patients we retrospectively observed. Median age was 46.5 months $(0.3-339)$, median bodyweight was $13.95 \mathrm{~kg}(3-88)$. We studied the relation of the percental change between two following measurements of GEDVI and indexed Stroke Volume (SVI). The same percental differences were calculated for central venous pressure (CVP) and SVI. Mean number of TIPD measurements per patient was 10 measurements (SD 4.3 , range $3-27$ ). We excluded 4 patients with less than 4 TIPD measurements and one patient with obvious data error. To avoid the risk of an interindividual bias between the patients with high and low numbers of measurements we performed regression and correlation analysis in each patient for GEDVI/SVI and CVP/SVI.

The pearsons correlation coefficients for CVP/SVI followed a normal distribution, so the mean average of the r-values was calculated for the 99 patients. The correlation coefficients of GEDVI/SVI did not follow a normal distribution, therefore the median of the $r$-values was generated. The median correlation coefficient $r$ (delta GEDVI/delta SVI) was 0.77 . The mean correlation coefficient r (delta CVP/delta SVI) was 0.17 .

We conclude that changes in GEDVI adequately reflect changes in cardiac preload in children after corrective cardiac surgery. We also conclude that changes in CVP do not sufficiently reflect changes of cardiac preload in these patients.

\section{P147}

Impact of an extended hemodynamic monitoring after corrective cardiac surgery in infants and children

Ch. Pauli, N. Egermann, U. Fakler, T. Genz, M. Hennig, J. Hess

Deutsches Herzzentrum München, Paediatric Cardiology, München, D

Our aim was to test whether an extended hemodynamic monitoring using the Transpulmonary Indicator Dilution (TPID) method is able to raise cardiac index after 48 hours postoperatively and may improve the course after cardiac surgery in infants and children.

In this prospective, stratified, randomised, controlled and blinded study we enrolled 30 patients. 16 patients in the study group, median age 24 months (1-210), median bodyweight $11.4 \mathrm{~kg}$ $(3.6-51.3)$ and 14 patients in the control group, median age 25.5 months (0.3-275.3), median bodyweight $11.1 \mathrm{~kg}(3.9-74.1)$. The operative treatments were comparable between the groups. We found no differences pre- and intraoperatively. After the admission on the ICU and then every 6 hours until 72 hours postoperatively. TPID measurements were performed in both groups by an independent examiner. Only the results in the study group for cardiac index (CI), global enddiastolic volume index (GEDVI), intrathoracical blood volume index (ITBVI), systemic vascular resistance index (SVRI) and extravascular lung water index (EVLWI) were presented to the responsible physician additionally to the routine hemodynamic monitoring data. Together with the clinical presentation the treatment was managed following standardised flow charts for augmentation and optimisation CI also taking into account the other parameters of TPID.

$\mathrm{Cl}$ after $48 \mathrm{~h}$ postoperatively did not differ between both groups. The median CI in the study group was $3.381 / \mathrm{min} \mathrm{m} 2(2.30-5.95)$ vs. $3.441 / \mathrm{min} \mathrm{m} 2(2.53-4.54)$ in the control group, $\mathrm{p}=0.85$. The median duration on the ICU was different. In the study group median $65 \mathrm{~h}(42-162)$ vs. $123.5 \mathrm{~h}(51-308)$ in the control group, $\mathrm{p}=0.008$. The cumulative dosis of vasodilators (nitrate) was higher in the study group ( $467 \mathrm{mg} / \mathrm{kg}$ vs. $203 \mathrm{mg} / \mathrm{kg}$ ), $\mathrm{p}=0.029$. We found no difference in other parameter.

We conclude that an extended hemodynamic monitoring with the additional measurement of CI, GEDVI, SVRI and EVLWI by TPID after corrective cardiac surgery in infants and children fails to raise cardiac index after $48 \mathrm{~h}$ postoperatively but reduces the stay on the intensive care unit significantly.

\section{P148}

Acute rheumatic fever in children: an 8-year experience

N. Ozbarlas, O. Canan, O. Kucukosmanoglu, S. Erdem,O.K. Salih University of Cukurova, Adana, TR

We reported our experience with acute rheumatic fever (ARF) during the years 1993-2001. Diagnosis was based on the modified Jones criteria. Of the 206 patients, 97 were girls while 109 were boys. The patients were between 5 and 16 years old with a mean of 11 years during the first attack. Manifestations included polyarthritis $(15 \%)$, carditis $(16.5 \%)$, chorea $(3.9 \%)$, polyarthritis + carditis $(58.3 \%)$, carditia + chorea $(3.9 \%)$, polyarthritis + carditis + chorea $(2.4 \%)$, fever $(73.8 \%)$, arthralgia $(76.2 \%)$. Of the patients with carditis, $61(36.5 \%)$ were considered to have severe carditis with congestive heart failure, $76(45.5 \%)$ were moderate carditis and $30(17.9 \%)$ were mild. Silent carditis was seen $7.0 \%$ of patients. Pericardial effusion occurred in $6.58 \%$ of patients. Mitral insufficiency and aortic insufficiency occurred in $63 \%$ and $5 \%$ respectively. Both mitral and aortic valves were involved $32 \%$ of cases. Tricuspid insufficiency developed in $1.7 \%$. Benzathine penicillin for the prophylaxis of streptococcus was recommended to all patients. 
The follow up period was 1-84 months. Carditis resolved completely in three patients $(1.7 \%)$ at the follow up and the recovery time was within 11-24 months. Mitral stenosis developed in $6.5 \%$ of patients. Recurrence was seen in $1.9 \%$ once, $0.9 \%$ twice. Nine patients underwent surgical intervention; mitral valve replacement was performed on seven patients and both aortic and mitral valve replacement in two patients. One patient died with congestive heart failure.

Acute rheumatic fever and rheumatic heart disease continue to be a major health problem in developing countries as Turkey.

\section{P149}

\section{Clinical and surgical findings in idiopathic right} atrial dilatation

M. Witsenburg, P.L. De Jong, A.J.J.C. Bogers, E. Meijboom

Sophia Children's Hospital/Erasmus MC, Rotterdam, NL

Background: Idiopathic right atrial (RA) dilatation is a very rare diagnosis with a variable clinical presentation.

Objective: We studied clinical and surgical findings in 3 patients (pts) whon were treated in recent years.

Results: Idiopathic right atrial dilatation, or giant right atrium, was diagnosed in one child and two adults. The diagnosis was made on prenatal echo in the child, on routine $\mathrm{X}$-ray at age five in one of the adults and because of arrhythmias in the other. In $2 / 3$ pts an erroneous diagnosis of Ebstein's disease was suspected at first. Limited exercise tolerance and palpitations were the major complaints in both adults. The ECG showed sinus rhythm in two and atrial fibrillation in one pt. Echocardiography and MRI/CT revealed massive dilatation of the RA with compression of the right ventricle in all. Doppler flow tracings showed abnormal tricuspid inflow patterns in $2 / 3$ pts. The patient with atrial fibrillation presented with two large thrombi in the RA. Surgical reduction of the giant RA was performed in both adults because of complaints, and electively in the non-symptomatic child. Follow-up ranged from $0.5-5$ years. Surgical treatment resulted in major clinical improvement of both adults and disappearance of the palpitations in one of them. Histology did not show a consistent morphological pattern. Doppler tricuspid flow after surgery showed a restrictive inflow in one pt.

Conclusion: Surgical reduction of idiopathic RA dilatation results in major improvement in symptomatic pts. Timing of surgical intervention in pts without complaints remains unclear but likely contributes in the prevention of arrhytmias and thromboembolic events.

\section{P150}

\section{Clinical signs of heart failure associated with} increased levels of P-BNP and P-ANP in children with congenital heart defects

A. Westerlind, D. Holmgren, G. Lindstedt, P. Lundberg, H. Whlander The Clinic of Internal Medicine, Lidkping, $S$

Introduction: Brain (BNP) and atrial natriuretic peptide (ANP), produced in the chambers of the heart, have been shown to increase in plasma during systolic dysfunction and different types of hemodynamic overload of the heart. Coarcatio aortae (CoA) leads to a pressure overload of the left ventricle, ventricular septal defect (VSD) to a volume overload of both ventricles while dilated cardiomyopathy (DCM) represents an overall decreased function of the left ventricle. Strain on the heart overwhelming compensatory mechanisms may result in clinical signs of heart failure (CSHF).
Aim: To study if P-BNP and P-ANP reflect CSHF in children with congenital heart defects and different types of hemodynamic overload.

Methods: Blood samples were obtained for analysis of P-BNP and P-ANP from 26 children (9 CoA, $11 \mathrm{VSD}, 6 \mathrm{DCM}$ ) during regular investigation preceding cardiac intervention. Reference values for the laboratory: $0-18.4 \mathrm{ng} / \mathrm{L}$ for $\mathrm{P}-\mathrm{BNP}$ and $0-43 \mathrm{ng} / \mathrm{L}$ for P-ANP. CSHF were evaluated from information obtained from the case records.

Results: $0 / 9$ children with CoA, 5/11 with VSD and 6/6 with DCM had CSHF. The median values for P-BNP and P-ANP were $7 \mathrm{ng} / \mathrm{L}$ (range 4.8-24.4) and $42.2 \mathrm{ng} / \mathrm{L}$ (range 13.7-63.2) in the children with CoA, $30.8 \mathrm{ng} / \mathrm{L}$ (range 10.7-81.5) and $166 \mathrm{ng} / \mathrm{L}$ (range 31.8-346) in children with VSI) and 639 $\mathrm{ng} / \mathrm{L}$ (range 263-1300) and $412 \mathrm{ng} / \mathrm{L}$ (range 198-466) in children with DCM respectively.

In children with CSHF, P-BNP and P-ANP were higher, $263 \mathrm{ng} / \mathrm{L}$ (range $47.5-1300$ ) and $303 \mathrm{ng} / \mathrm{L}$ (range 168-466), than in those without CSHF, $12,3 \mathrm{ng} / \mathrm{L}$ (range $4.8-30.8$ ) and $42.9 \mathrm{ng} / \mathrm{L}$ (range 13.7-189), respectively $(p<0.001)$ (Mann-Whitney $\mathrm{U}$ test), irrespective of the diagnosis. The same relationship was also found within the group of children with VSD.

Conclusion: Both P-BNP and P-ANP were increased in children with CSHF. The highest values were seen in those with DCM. An isolated pressure or volume overload of the heart was associated with a certain increase in P-BNP and P-ANP which was significant when CSHF was present. Thus, P-BNP and P-ANP seem to reflect CSHF in children with congenital heart defects.

\section{P151}

Quantification of regional right and left ventricular function by ultrasonic strain rate and strain indices after surgical repair of tetralogy of Fallot

L. Mertens, F. Weidemann, B. Eyskens, D. Boshoff, C. Dommke, M. Kowalski, B. Bimens, L. Hatle, G.R. Sutherland, M. Geurillig University Hospitals Leuven, Leuven, $B$

Background: The quantification of regional myocardial function in Tetralogy of Fallor (TOF) by conventional M-mode and 2-D echocardiography is difficult because of the complex morphology of the right (RV) and the altered geometry of the left ventricle (LV) Methods: In 30 asymptomatic post-operative TOF patients (4-16 years) with low pressure RV's and with varying degrees of pulmonary regurgitation and in 30 aged-matched healthy children, the ultrasonic derived regional deformation parameters peak systolic strain rate (SR) and systolic strain (e) were acquired from both ventricles and compared.

Results: In TOF, RV free walls, SR and e were reduced in the basal, mid and apical segments and averaged $-1.50 .6 \mathrm{~s}^{-1}$ for SR and $-228 \%$ for e respectively ( $p<0.001$ vs normals). Peak systolic SR of the basal RV free wall correlated significantly with the QRS-duration of the ECG $(r=0.81 ; p<0.0001)$. Abnormalities in RV deformation were more marked in those patients with transannular patches versus infundibular patches. In the septum there was a homogeneous reduction in SR/e in the basal, mid and apical segments. These averaged $-1.40 .3 \mathrm{~s}^{-1}$ for SR and $-194 \%$ for e respectively ( $\mathrm{p}<0.01$ vs. normals). Longitudinal SR/e values of the three LV lateral wall segments (averaged $\mathrm{SR}=-1.60 .4 \mathrm{~s}^{-1}$, averaged $\mathrm{e}=-205 \% ; \mathrm{p}<0.05$ vs. normals) and radial $\mathrm{SR} / \mathrm{e}$ of the $\mathrm{LV}$ posterior wall $\left(\mathrm{SR}=3.30 .9 \mathrm{~s}^{-1} ; \mathrm{e}=5114 \% ; \mathrm{p}<0.05\right.$ vs. normals) were significantly reduced.

Conclusions: In this study, abnormalities in regional systolic myocardial function in the $\mathrm{RV}$ and $\mathrm{LV}$ in asymptomatic 
post-operative TOF patients were quantified by the deformation parameters $\mathrm{SR}$ and $e$. The RV deformation abnormalities are associated with electrical depolarization abnormalities. The ultrasonic measurement of regional deformation parameters may add to our understanding of the progression of residual abnormalities of myocardial function in this patient group.

P152 (see Abstract 47)

Longterm follow-up after Fontan operation for tricuspid atresia: The Dutch Experience

R. Aydinli,J. Ottenkamp, A. Cromme-Dijkhuis, T. Hoorntje,

R. Tanke, N. Veeger, J. Strengers

Academisch Medisch Centrum, Amsterdam, NL

\section{P153}

Effect of cardiopulmonary bypass on myocardial function, damage and inflammation after cardiac surgery in newborns and children

S. Hammer, M. Loeff, S. Daebritz, H. Reichenspumer,

R. Kozlik-Feldmann, B. Reichart, H. Netz

Department of Pediatric Cardiology, Klinikum Grohadern, Munich, $D$

Children are at risk for developing myocardial injury associated with cardiac dysfunction after cardiac surgery with cardiopulmonary bypass and prolonged aortic cross clamping time (CCT). This clinical symptom usually manifests in impaired cardiac function with low cardiac index because of reduced myocardial contractility and compliance. The assessment of the degree of myocardial damage is difficult to ascertain in the postoperative period. To clarify these issues we evaluated cardiac Troponin I (cTnI), Creatine Kinase (CK) and Procalcitonin (PCT) levels in infants experiencing uncomplicated repair of congenital heart disease. The purpose was to establish patterns of elevation and to assess the degree of myocardial damage and inflammation after cardiac surgery with cardiopulmonary bypass in children.

The elevation of cTnI, CK and PCT was evaluated in relationship to duration of aortic cross-clamping, incisional trauma and cardiac bypass temperature in 37 pediatric patients. CTnI and PCT both peaked on the day of surgery and decreased postoperatively in case of an uncomplicated course. The median values of both parameters differed significantly from the day of surgery until the fourth postoperative day in children with CCT longer than 80 minutes or after ventriculotomy in comparison to patients with shorter clamping times or atriotomy only. $\mathrm{CK}$ values showed similar results but were less significant than $\mathrm{C} T \mathrm{nI}$. No relationship was found between cTnI, CK or PCT and body temperature during cardiopulmonary bypass.

In summary, perioperative measurement of $\mathrm{CTnI}$, CK and PCT reflects myocardial damage and systemic inflammatory response and allows an improved peri- and postoperative management.

\section{P154}

\section{Is cardiorespiratory capacity reduced after corrective} surgery for tetralogy of Fallot?

A. Heusch, M. Reitz, M. Deuen, K. G. Schmidt

Pediatric Cardiology, Heinrich-Heine-University, Duesseldorf, D

After corrective surgery for tetralogy of Fallot (TOF) many patients present with impaired RV-function which may lead to reduced cardiorespiratory capacity. The objective assessment of cardiorespiratory capacity is possible by exercise testing with continuous $\mathrm{O}_{2}$-uptake measurement. Using this approach we studied cardiorespiratory capacity in 38 patients after TOF repair.
Maximal oxygen uptake $\left(\mathrm{VO}_{2}\right.$ max), carbon dioxide output $\left(\mathrm{VCO}_{2}\right)$, anaerobic threshold ( $\left.\mathrm{AT}\right)$, heart rate (HR), and blood pressure (BP) were measured during treadmill testing using a modified Bruce-protocol.

22 male and 16 female TOF patients (mean age 12.3 years. range 6-18) were studied after a mean postoperative follow-up of 9.83.4 years. The results, subdivided by type of surgical intervention (sub- or transannular patch) were compared with those of an aged-matched control group $(n=47)$.

TOF patients had a shorter exercise time (12.12.4 vs. $14.32 .3 \mathrm{~min}$ : $\mathrm{p}<0.001)$ and a lower $\mathrm{VO}_{2} \max (29.77 .0$ vs. $41.98 .5 \mathrm{ml} / \mathrm{kg} / \mathrm{min}$. $\mathrm{p}<0.01)$ than control subjects. AT was reduced in TOF patients $(17.45 .1$ vs. $23.62 .3 \mathrm{ml} / \mathrm{kg} / \mathrm{min}, \mathrm{p}<0.01)$. There was no difference between groups in response to heart rate or blood pressure to exercise. With respect to type of surgery patients with transannular patch showed shorter exercise time $(10.41 .5$ vs. $12.52 .3 \mathrm{~min}$. $\mathrm{p}=0.01$ ) than those with transannular patch but no difference in $\mathrm{VO}_{2}$ max and AT.

We conclude that that TOF patients show a normal rise in heart rate and blood pressure but have a reduced cardiorespiratory capacity in mid-term follow-up after repair. Reduction of cardiorespiratory capacity appears to be similar in patients with sub- and transannular patch.

\section{P155}

Specific features of cardiac manifestations in malignant lymphoma in child

G. Dimitriu, I. Miron, T. Condurache, D. Mihails, A. Mad

I-st Clinic of Pediatrics, Iasi, Romania, $R$

The aim of the study was to present the most important cardiac manifestations, other than those caused by the side effects of the specific therapy, in children with a malignant lymphoma (ML).

Methods: Patients: 14 children aged between 2 and 16 years diagnosed with a malignant lymphoma with cardiac involvement: 12 of them with a nonHodgkin lymphoma (NHML) and 2 cases with Hodgkin disease. The cardiac clinical signs were correlated with the ECG, chest X-ray and echocardiographic (Echo) findings.

Results: Cardiac involvement was observed in $5 \%$ of NHML indifferent of the inital localisation of the tumor and the stage of the disease. The clinical signs were often non-specific and usually were attributed to the malignant disease: chest pain, dyspnoea, superior vena cava syndrome. The main cardiac manifestations proved by Echo were: pericardial effusion (10 cases), serousfibrinous ( 4 cases) or haemorrhagic fluid $(6$ cases) up to cardiac tamponade ( 3 cases), with the presence of malignant lymphomatous cells in the pericardial fluid; pericardial tumour ( 2 cases): myocardial diffuse infiltration by lymphoma (1 case), but without clinical signs. The specific therapy leaded, in general, to the improvement of the cardiac disorders, improving, at the same time, the immediate evolution of these patients, without improving the prognosis of the main disease, though.

Conclusions: Cardiac involvement is frequently present in children with $M L$, independent of specific therapy, but clinical signs are often non-specific and cardiac manifestations may be severe and may worsen the prognosis. This impose a systematic cardiological investigation, especially by Echo in all the stages of the disease and during the evolution. Spotlighting of malignant cells in the pericardial liquid, correlated with the clinical and radiological findings, can establish the diagnosis of NHML in those cases in which the biopsy could not be performed (mediastinal location), allowing to start the specific therapy. 
P156

Immunosuppressive drug combination therapy in the treatment of recurrent pericarditis

L. Zavota, G. Ricciardi, B. Teliana, A. Agnetti, N. Carano,

F. de Leva, L. Squarcia

Parma Hospital - Department Of Pediatrics, Pama, I

Aim of the study: The recurtent pericarditis in pediatric age group are due in the majority of instances to an autoimmune mechanism. The aim of our communication is to present a rational polypharmacologic treatment protocol, already used in our two centers, for cases resistent to the standard anti-inflammatory therapy, in order to increase the effectiveness and reduce the risks of side effects.

Methods: Our experience is based on 10 children ( 7 boys and 3 girls) enrolled at the second episode of acute pericarditis, with the follow-up ranging from 1 to 6 years. At the time of enrolment, all the patients where test to exclude secondary forms.

Methylprednisolone was given e.v. bolus $(3-5 \mathrm{~m} / \mathrm{kg} / \mathrm{dose})$ and repeated, if necessary, 2-3 times every other day at the time of the first episode and at the first signs of relapse, event if only clinical. If there was a positive effect, the treatment was continued with low doses of steroids p.o., prednisolone or fluocortolone $0,5-1 \mathrm{mg} /$ $\mathrm{kg} /$ week up to a maximum dosage of $40 \mathrm{mg} /$ week, in 4 non consecutive days. In case of negative response or when the relapses are more than once a month at the low dose steroid therapy we add cyclosporin and if necessary methotrexate as third drug.

The treatment was gradually tapered after six months without relapses until complete suspension of the therapy.

Results: The age of the first diagnosis varies from 18 months to $1+$ years. The number of relapses ranges from 0 to 6 (mean 2,5). Autoantibodies were found in 5 cases $(50 \%)$. In $3(30 \%)$ the treatment with only steroids was effective; in 5 cases $(50 \%)$ two drugs were used; in other $2(20 \%)$ the association of three drugs (steroids + cyclosporin + methotrexate) had been necessary. Conclusions: The treatment according to this new protocol had been effective in all of our cases and without remarkable side effects. At this time all our patients are in remission.

\section{P157}

Intelligent system for cardiac diseases decision

making in the young

P. Kokol, M. Zorman, M. Molansiglic

Lniversity of Maribor, Maribo, SLO

As in many other areas, predictions play an important role in health care. What will be the status of the patient after the selected treatment, will the healing process end with the success or not are just some of the possible predictive questions faced by both patients and medical staff. Statistics can be of great help in many real world situations, but sometimes its use is limited. As an alternative we can use so called intelligent data analysis techniques like neural networks, case based reasoning, logic programming, or decision trees. The greatest weakness of the majority of these techniques is that they are black box approaches - normally giving good predictions but without explanation. One of the techniques overcoming this fault is the decision tree approach, which in addition to immense prediction power also explains the decisions leading to the predictions using a simple two-dimensional graphical model.

We used the decision tree approach in the intelligent analysis of cardiac diseases in the young. We analysed the pediatric record for 147 patients with our intelligent system and compared the results with the decisions of pediatricians. We got excellent results, with the overall diagnostic accuracy over 91 percent. In addition some new diagnostic rules and guidelines have been extracted.
Thereafter our claim is that intelligent decision support systems can help medical stuff in the decision making process, especially if they are not specialised in cardiology or pediatrics.

\section{P158}

Effects of heart surgery in school-aged children on neurocognitive and perceptual-motor functioning R. Vand de Rijken, I. Hulstijn-Dirkmaat, M. Bos, B. Maassen, A. Nijueld, O. Daniels

Nimegen, $\mathrm{NL}$

Introduction: Recent studies on the effects of complex heart surgery demonstrated that cardiac surgery in school-aged children will not have measurable effects on global indices of intelligence but might have a negative impact on neurocognitive and perceptual-motor functioning. To identify the distinctive contribution of pre-, peri-, and post-operative risk factors to these adverse effects, we started a prospective study in patients aged 6-16 yrs. with CHD and indicated for heart surgery. Here we present the results of a pilot study.

Patients: Twelve patients indicated for elective surgery (10 boys, 2 girls) met the inclusion criteria (open-heart surgery, no comorbidity). The mean age was $11.7 \mathrm{yrs} .(6.3-15.4)$. The type of disease and surgical procedure varied from simple $(\mathrm{n}=2$; ASD) to complex $(n=10$; univentricular and cyanotic condition, arch anomalies, reoperation with major involvement of the circulatory system).

Methods: Two assessments were performed: T1: pre-operative, T2: 1 yr. post-operative. The cognitive status was assessed by the WISC-R; neurocognitive functioning by the Stroop and Bourdon-Wiersma-Vos tests for children; perceptual-motor functioning by the Visual Motor Integration task and by special drawing tasks using hardware and software (OASIS) to record and analyse the kinematic aspects of the movements.

Results: Cognitive functioning in the pre-operative assessment was at a subnormal level (mean total IQ 94.4). No significant differences were found in the post-operative condition, although two patients improved considerably and two patients showed a remarkable decrease. Neurocognitive functioning: the Stroop test showed a decrease in the post-operative measurement in 3 patients. On the Bourdon task half of the patients were slow, but all improved slightly from T1 to T2. Perceptual-motor functioning: the VMIstandard scores in the pre- and post-operative assessments were normal. The drawing tasks showed a significant impaired performance in the pre-operative condition and a slight improvement in 8 patients post-operatively. With regard to medical risk factors a poor pre-operative condition (low $\mathrm{pH}$ and low $\mathrm{Hb}$ ) and a long duration of ECC were only noticed in the two patients with a post-operative decrease on several tests.

Conclusion: The neurocognitive and perceptual-motor tasks did provide important information to assess the effects of the operation.

P159 (see Abstract 23)

Cardiac manifestations and complications in children with the acquired immunodeficiency syndrome at the Chris Hani Baragwanath Hospital, Johannesburg

F.F. Takawira, A.M. Cilliers

Chris Hani Baragwanath Hospital, Johamesburg, RSA

P160 (see Abstract 46)

Anti-RO and anti-LA antibodies and their effect on conduction in the absence of overt heart block - a prospective study

P. Gordon, M. Khamashta, G.R.V. Hughes, E. Rosenthal, J. Simpson, G. Sharland

Guy's and St. Thomas' Hospital, London, $U K$ 
P161 (see Abstract 51)

Prevalance and spectrum of cardiac abnormalities in pediatric patients with intestinal malrotation.

E. Thomas, A.P. Salmon, B.R. Keeton, J.P. Gnanapragasam,

J.J. Vettukattil

Southampton General Hospital, Southampton, UK

\section{P162}

Longterm cardiological outcome after neonatal arterial switch operation (ASO) for transposition of the great arteries (TGA)

H.H. Hvels-Grich, M. - C. Seghaye, D. Kunz, M. Miskova,

B.J. Messmer, G. von Bernuth

Department of Paediatric Cardiology, Univ.Hosp., RWTH Aachen, Aachen, D

The purpose of this prospective longitudinal study was to assess cardiological status and exercise capacity of children between 8 and 14 years of age after neonatal ASO for TGA.

60 unselected children with intact ventricular septum (78.3\%) or ventricular septal defect $(21.7 \%)$ without or with aortic isthmic stenosis (5.1\%) underwent, at mean age 10.51.6 (mSTD) and 5.3 years after midterm evaluation, clinical examination, standard and 24 h Holter electrocardiogram (ECG), echocardiography, Bruce walking treadmill test and determination of cardiac serum markers troponin I and T, creatin kinase and myoglobin at rest and $4 \mathrm{~h}$ after exercise. Results were compared to normal values and to midterm follow-up results.

Rates of reoperation and second operation to correct coarctation were $3.3 \%$ and $5.1 \%$, respectively. $90 \%$ had normal exercise capacity, reduction in $3.3 \%$ was not due to cardiac disease, $6.7 \%$ could not be staged. No patient needed medication. $31.6 \%$ had abnormal thoracic configuration after median sternotomy.

Most patients (91.7\%) were in sinus, the others $(8.3 \%)$ in ectopic atrial or junctional rhythm. Compared to midterm follow-up, I and intermittent II heart block increased to $6.7 \%$ and $3.3 \%$, respectively. Incidence of complete right bundle branch block $(10 \%)$ and ectopic activity (atrial 20.0\%, ventricular $21.7 \%$ ) were unchanged. During and after exercise, ECG remained normal in $90 \%$; coronary artery stenosis was excluded by coronarography in $3.3 \%$ showing ST-T segment depression or frequent ventricular ectopy. Levels of cardiac markers were normal in all patients.

Left ventricular function did not deteriorate over time. Diameters of aortic valve annulus and root were in $10 \%$ and $63 \%$, respectively, beyond normal values; both diameters did not increase, compared to midterm follow-up. Aortic insufficiency was correlated to root dilatation, its incidence $(13.3 \%)$ was unchanged. Compared to midterm follow-up, incidence $(41.6 \%)$ and degree of supravalvular pulmonary stenosis increased.

The study points out persistent good cardiological results 10 years after neonatal ASO for TGA. Preservation of left ventricular function, low incidence of rhythm disturbances, normal exercise capacity, lack of further aortic root dilatation and unchanged incidence of aortic insufficiency, compared to midterm follow-up, are encouraging. Increased incidence and degree of supravalvular pulmonary stenosis are of concern.

\section{P163}

Progressive dilation of the ascending aorta in patients with Turner Syndrome

J.B. Thambo, A. Fournier, J.L. Bigras, J. Thrien, C. Deal

Hôpital Sainte-Justine, Montral, CDN

Cardiac anomalies are a well known feature of patients with Turner Syndrome (TS). These include dilation of the ascending

aorta, with dissection leading to unexpected sudden death in rare cases. Current AAP guidelines (Pediatrics 1995; 96:1166) recommend cardiac ultrasounds yearly, however no longitudinal data on aortic dilation exist, and no recommendation on the timing of medical or surgical treatment are available. We undertook a retrospective review of the cardiac ultrasounds in patient with TS seen in our institution by the Endocrinology Service between 1992 and 2000

Of 105 girls, $25 \%$ came with report of a normal ultrasound performed elsewhere. Seventy-nine girls $(75 \%)$ underwent at least one cardiac evaluation at our center, $64 / 79$ were seen more than once. Mean age (SD) at initial evaluation was 9.45 .9 years (range 4 days to 22.11 years). Abnormal cardiac findings included: bicuspid aortic valve (9), aortic stenosis (6), mitral stenosis (2), mitral insufficiency (3), coarctation of the aorta (8), secundum atrial septal defect (3). Dilated ascending aorta was seen on at least 1 ultrasound in 53 patients $(67 \%)$ ( $Z$ score $>2$ compared to normal, size-matched controls), 35/79 (44\%) without associated cardiac anomaly. Mean $Z$ score of the aorta at initial examination was 1.98 1.0. At last evaluation (mean duration of follow-up 5.53 .2 years), the $Z$ score was 2.50 .96 , an increase of $5292 \%$. Treatment with growth hormone did not influence the progression of the dilation. Patients had a higher blood pressure with a mean $\mathrm{Z}$ score of systolic blood pressure of $1.301 .18, p<0.0001,10 / 59(17 \%)$ Z score $>2$.

In conclusion, aortic dilation is a frequent finding in TS who have a known cardiac anomaly but it can also be encountered as an isolated finding. Dilation is progressive with time. Close follow-up of these patients is mandatory.

\section{P164}

Isolated noncompaction of left ventricular myocardium in children

R. Grillo, S. Pipitone, M. Mongiov, T. Cipolla, G. Giudice,

S. Gagliano, V. Sperandeo

Ospedale Casa del Sole - Palermo, Palermo, I

Isolated noncompaction of the left ventricular myocardium, a rare congenital disorder, is thought to be due to an arrest of myocardial morphogenesis. It is characterized by excessively prominent trabecular meshwork and deep intertrabecular recesses. In this report we describe the clinical, electrocardiographic and echocardiographic findings on 5 paediatric cases, with age ranging from 1 month to 10 years. These patients have been observed with a mean follow-up of 4,5 years (range 1-11 years). Life-threatening ventricular arrhythmias and systemic embolic events never occurred. Four patients with heart failure have been treated with digoxin, diuretics, angiotensin-converting enzyme and anticoagulant therapy, and one of these is still asymptomatic and show good physical fitness. One patient died while waiting for cardiac transplantation. Early diagnosis and appropriate treatment of this disorder can improve the outcome, even though further study will be need to elucidate its long-term prognosis. At the same time owing to high incidence of family recurrence, evaluation of relatives is warranted.

\section{P165}

Plasma concentrations of $\mathrm{N}$-terminal pro-Brain Natriuretic Peptide (N-BNP) in healthy children and in children with heart diseases

T.S. Mir, S. Marohn, S. Laer, M. Eiselt, C. Haun, M. Flato, J. Weil

University Hospital Hamburg, Hamburg, D

Objective: To determine plasma levels of N-BNP in children with or without heart disease to support guidance of clinical therapy in 
uture. Reference values and the clinical importance of N-BNP in hildren has not yet been studied.

Methods: In 23 children ( 2 months-14 years) with congestive teart failure (CHF) plasma levels of N-BNP were correlated with he ejection fraction and a clinical score for children with CHF. In ddition N-BNP was measured before and up to 15 days postsperatively in 20 children ( 4 month-11 years) undergoing cardiac urgery. Dosage and duration of catecholamines, vasodilators and espiratory therapy were documented. For comparison, plasma evels of N-BNP were measured in 133 children and adults of diferent age groups ( 11 days to 32 years) without heart disease using validated enzyme immunoassay.

2esults: The 23 children with heart failure had increased N-BNP evels of $762(219-2008 ; \mathrm{p}<0.05) \mathrm{fmol} / \mathrm{ml}$. N-BNP levels correated significantly with the heart failure score $(r=0.57 ; p<0.01)$. $\mathrm{n}$ addition the ejection fraction showed a negative correlation to $\checkmark$-BNP values $(r=0.55 ; p<0.05)$. We found a positive corelation $(r=0.77 ; p<0.01)$ between maximal N-BNP levels and losage of vasodilators in children undergoing cardiac surgery. Josage and duration of catecholamines and respiratory therapy vere not correlated to pre- or perioperative N-BNP levels. $\checkmark$-BNP levels in children, adolescents and adults without heart lisease did not show any significant age related differences. Levels selow $430 \mathrm{fmol} / \mathrm{ml}(90 \%$ percentile) were regarded as normal for Jaediatric patients beyond the first 10 days of life.

nterpretation: N-BNP reflects the extend of symptoms of heart ailure and the impairment of heart function in paediatric patients with congestive heart failure. The value of N-BNP for guiding serioperative therapy on a peadiatric cardiac intensive care unit ieems to be low. In children without heart diseases reference valles for N-BNP were defined.

\section{P166}

Evaluation of bone mineral density in infants with zyanotic congenital heart disease

Dr. M. K. Lenk, Dr. V. Okutan, Dr. S. mit sars, Dr. S. Ilgan,

Dr. D. Gkay

Glhane Military Medical Academy Dept. of Ped. Cardiology,

Ankara, TR

Aim: Malnutrition and the resultant growth retardation are common problems in children with congenital heart disease (CHD). In this study growth retardation was clinically evaluated in infants with CHD by recording growth parameters including body weight and length, and head circumference and by making bone mineral density (BMD) measuruments in cyanotic, acyanotic and healthy (control) infants; and the relationship between the BMD values and the growth retardation, and hypoxemia was investigated.

Methods: BMD measurements were performed at L2-L4 lumbar vertebrae by "Dual Energy X-R ay Absorbtiometry" in 20 cyanotic, 25 acyanotic and 30 healthy infants with ages ranging between 2 to 12 months.

Results: Thirteen (28\%) of the 45 cases with CHD were found to have body weight below third centile whereas $9(20 \%)$ of these cases had both body weight and length below third centile. Cyanotic and acyanotic patient groups had significantly less body weight when compared to control group, and body length was significantly shorter in cyanotic patient group when compared to the other two groups. BMD measurements of the acyanotic CHD group did not differ significantly from those of the control group whereas BMD values of the cyanotic CHD group were significantly lower than those of the other two groups. This difference was more prominent in those cases with body weight and length below the third centile.
Investigation of the relationship between BMD values and severity of hypoxemia revealed that $\mathrm{BMD}$ values were significantly lower in cases with $\mathrm{PaO}_{2}$ of $<40 \mathrm{mmHg}$ and $\mathrm{SaO}_{2}$ of $<80 \%$, and that $\mathrm{BMD}$ values increased with the increasing values of oxygen pressure and saturation

Conclusion: We conclude that hypoxemia may cause growth retardation and may lower $\mathrm{BMD}$ values in infants with $\mathrm{CHD}$, and that palliative or corrective measures should be performed in these cases to prevent growth retardation reducing the severity of hypoxemia.

\section{P167}

The efficacy of IVIG in prevention of giant coronary artery aneurysm in Kawasaki disease

V. Jamiureeruk, W. Tantiprasit, C. Sangtaweesin, T. Layangool, T. Kirawittaya

Bangkok Heart Institute, Bangkok General Hospital, Bangkok, T

Kawasaki disease can cause cardiovascular complication especially coronary artery aneurysm which is the main cause of death. Up to now, the standard treatment is intravenous immunoglobulin (IVIG) with aspirin. This regimen can reduce both the morbidity and mortality including the giant coronary artery aneurysm.

The purpose of this study is to evaluate the efficacy of the appropriate dose and time of IVIG and aspirin can prevent the giant coronary artery aneurysm or not.

Material and method: The retrospective study was performed from January, 1992 to December, 1999 at the Queen Sirikits National Institute of Child Health. There were 71 cases who had received the appropriate dose of IVIG, either $2 \mathrm{gm} / \mathrm{kg} / \mathrm{single}$ dose or $400 \mathrm{mg} / \mathrm{kg} /$ day for 4 consecutive days with aspirin 80 $100 \mathrm{mg} / \mathrm{kg} /$ day before the ninth day of illness were enrolled in this study. There were 52 boys and 19 girls. The ages ranged between 4-73 months (mean 20 months). 65 cases had received IVIG $2 \mathrm{gm} / \mathrm{kg}$ as a single dose and 6 cases had received $400 \mathrm{mg} / \mathrm{kg} / \mathrm{day}$ for 4 consecutive days with aspirin $80100 \mathrm{mg} / \mathrm{kg} /$ day within the ninth day of disease.

Results: 7 cases $(9.8 \%$ ) had cardiac complication (coronary artery aneurysm). 3 cases (4.2\%) had small coronary artery aneurysm, 1 case $(1.4 \%)$ had a moderate coronary artery aneurysm and 3 cases (4.2\%) had giant coronary artery aneurysm

The incidence of giant coronary artery aneurysm was $4.2 \%$ of Kawasaki disease patients or $42.8 \%$ of Kawasaki disease patients with coronary artery aneurysm. This seemed to be very high when compared to other reports. Two of these 3 cases of giant coronary artery aneurysm were treated with IVIG $2 \mathrm{gm} / \mathrm{kg}$ (one case had IVIG two doses) and another one was treated with $400 \mathrm{mg} / \mathrm{kg} / \mathrm{day}$ for $4 \mathrm{con}-$ secutive days. One case had a massive clot in the LAD aneurysm. Conclusion: The appropriate dose and time of IVIG with aspirin treatment of Kawasaki disease may not prevent giant coronary artery aneurysm.

\section{P168}

Impact of right ventricular size on ECG after ASD device closure

S. Di Bernardo, F. Berger, M. Fasnacht, U. Arbenz, U. Bauersfeld Kinderspital Zrich/Kardiologie, Zrich, $\mathrm{CH}$

Prolonged QRS duration may predict adverse arrhythmic events in patients after tetralogy of Fallot repair. The different impact of cardiac surgery and right ventricular (RV) dilation on QRS duration is unknown. We studied the influence of RV size on QRS duration after nonsurgical atrial septal defect (ASD) device closure 
Methods: In 24 children aged $8.9+3.5$ years an ASD was closed with an Amplatzer device (mean device size $18+4 \mathrm{~mm}$ ). ECG and 2-D echocardiography were obtained before ASI) closure, 1 day and 1 month after intervention respectively. As measure for $R V$ size changes the percentage of right compared to left ventricular dimension was calculated. All children had an enlarged RV before intervention.

Results: While a significant decrease in $\mathrm{RV}$ size was even observed one day after ASD closure significant shortenings of QRS and QTc duration were seen just 1 month after intervention.

Conclusion: ASD device closure results in a fast and significant decrease of RV dimension and QRS duration. The decline of RV dimension precedes the alleviation of QRS duration after ASD device closure. Thus, QRS prolongation may be a consequence of $\mathrm{RV}$ dilation in children and may be used as a predictor for $\mathrm{RV}$ size in long term follow-up.

\begin{tabular}{lccc}
\hline & Before & 1 day & 1 month \\
\hline QRS (ms) & $87+13^{\star}$ & $83+11$ & $81+10^{\star}$ \\
QRS axis $\left(^{\circ}\right)$ & $72 \pm 32$ & $67 \pm 36$ & $6+ \pm 29$ \\
QTc (ms) & $437+15^{\star}$ & $424+20^{\#}$ & $+27+25^{\#}$ \\
RV/LV (1\%) & $96 \pm 11$ & $87 \pm 11^{\#}$ & $78 \pm 7^{\#}$ \\
\hline
\end{tabular}

${ }^{\star} \mathrm{p}<0.05$ before vs. 1 month, ${ }^{*} \mathrm{p}<0.05$ before vs. 1 day and 1 month.

\section{P169}

Long term heparin treatment of protein losing enteropathy in a child with heterotaxy after Fontan procedure

H. Knicuald, M. Jehusic, \. Rojnic Putarek, W.M. Novick, I. Malci University Hospital Rebro, Zagreb, CRO

Protein-losing enteropathy is a serious complication after Fontan procedure with high mortality rate and, by now, unknown pathogenesis. Several successfully treated cases of PLE with high molecular weight heparin have been reffered to in literature. We present the case of a 10-year old girl who developed PLE and has been treated with HMWH for a period of 50 months.

We present a girl with the initial diagnosis of heterotaxia syndrome (dextrocardia, single inlet ventricle of undetermined type, single AV valve, pulmonary atresia, hemyazygos vein continuity). The first palliative operation of Blalock-Taussig shunt on the right side was performed in early infant age, followed by bidirectional Glenn anastomosis at the age of 1 . At the age of 3 , Fontan procedure with fenestration was performed. Four months later the child developed PLE with typical clinical and laboratory signs: chronic pleural effusions, hepatomegaly, edema, hypoalbuminemia, hypocalcemia, lymphopenia and enteral losing of proteins (elevated levels of alfa1-antitripsin in stool). Clinical condition was improved after reoperation on cardiopulmonary bypass due to stenosis of pulmonary arteries, but after 3 months the PLE developed again. In June 1997 the therapy with HMWH was started with the initial dose of $8000 \mathrm{i} . \mathrm{j} . / \mathrm{m} 2$ with gradual increasement of the dose until clinical achievement and normalization of laboratory findings were achieved after 5 months with a dose of $15000 \mathrm{i} . \mathrm{j} . / \mathrm{m} 2$. Due to HMWH therapy and PLE, the child developed sacrodynia with the possibility of lumbal vertebrae fracture. Bone densitomerty showed a significant reduction of bone density, and Rocaltrol and elementary calcium were introduced in therapy. Every attempt to lower HMWH dose ended in worsening of clinical condition so, the therapy of 15000 i.j. $/ \mathrm{m} 2$ has been continued. Total protein scrum level is $45-50 \mathrm{~g} / \mathrm{L}$, with albumin fraction of $20-25 \mathrm{~g} / \mathrm{L}$. Serum calcium level is kept inside normal values.
Conclusion: Long-term PLE treatment after Fontan (TCPC) procedure is possible with high doses of $\mathrm{HMWH}$, with prevention of bone demineralization with vitamine $\mathrm{D}$ and calcium. Besides adequate high level protein, low fat diet is required.

\section{P170}

Hemodynamic monitoring by pulse contour analysis after cardiac surgery in infants and children

M. Loeff, S. Hammer, S. Daebritz, R. Kozlik-Feldmann,

B. Reichart, H. Nerz

Department of Pediatric Cardiology, Munid, D

The aim of our study was to optimize the minimal invasive management in children in the first 48 postoperative hours after cardiac surgery.

We measured cardiac index (CI), intrathoracic blood volume index (ITBVI) and systemic vascular resistance index (SVRI) with PiCCO-technique (pulse contour cardiac output, PULSION) in children with a mean age of 4932 months and a mean weight of $13+\mathrm{kg}$.

The continuous monitoring of these parameters led to precise administration of either catecholamines or fluid to maintain normal cardiac indices $(3,5-4,5)$.

There was a high correlation between CI and IT BVI $(r=0,9)$ as well as CI and SVRI $(r=0,8)$. In contrast the central venous blood pressure was not suitable for measurement of deviation from normovolemia.

$\mathrm{PiCCO}$ is a minimal invasive assessment of high clinical value for continuous monitoring of volume status and hemodynamics after cardiac surgery.

\section{P171}

QTe interval prolongation and QTc dispersion in

type 1 diabetic children and adolescents

B. Suys, D. De Wolf, S. Huybrechts, R. Rooman, B. Van Overmeire,

D. Mathly's

Linversity Hospital Antwerp, Edegen - Autuerp, B

Background: Several recent studies describe a relation between QTc prolongation, diabetic complications and an increased mortality in adults with diabetes.

Objective: To evaluate whether QT, QTc and QTc dispersion changes are already present in diabetic children and adolescents.

Study design: QT, QTc and QTc dispersion were measured on a 12 lead surface ECG in 60 children and adolescents with stable type 1 diabetes, and in 63 sex and age matched controls. Differences were evaluated using the Kolmogorov-Smirnov Z test. The number of patients with QTc $>4+0 \mathrm{~ms}$ was compared in the two groups. The possible influence of age, gender, diabetes duration and $\mathrm{HbAlc}$ was examined using Spearman correlation analysis.

Results: Diabetic children had significantly longer QTc intervals and a significantly larger QTc dispersion. The number of individuals with a $Q T c>440$ ms was significantly higher in the diabetic group $(14 / 60)$ than in the control group $(2 / 63)$. The effect of age on $\mathrm{RR}$ interval and $\mathrm{QT} \mathrm{C}$ dispersion in normal children was less pronounced in diabetic children. HbA1c values did not significantly correlate with any of the parameters.

Conclusion: QTc prolongation and a larger QTc dispersion are already present in a significant proportion of children and adolescents with diabetes. Prospective follow-up is needed to determine if these findings implicate a higher risk of arrhythmias or sudden death, as mentioned in adult diabetic patients. 


\section{P172}

Does prematurity predispose to pulmonary vein stenosis? R.S. Bader, S.Y. Ho, M. Josen, E.A. Shineboume, M.N. Shepperd, M.L. Rigby

Hospital for Sick Children, Toronto, Ontario, CDN

Pulmonary vein stenosis (PVS) in normally connected veins is rare, and leads to progressive pulmonary hypertension and cardiac failure in infancy. 8 consecutive patients with this condition presented between June 1982 and July 2000.

Age at diagnosis ranged from 1 day to 2.5 years. Initial diagnosis was made by echocardiography (and confirmed by catheterisation) in $4(50 \%)$ patients. The diagnosis was made at the initial cardiac assessment in 2 patients but was identified subsequently in 6 at a median of 3 months after referral. 3 patients were born prematurely, age at referral from 5-6 months, at a corrected age of 2 to 3.2 months, and weight from 2 to $4.3 \mathrm{~kg}$. 2 of the 3 premature infants died 1.5 to 3 months after diagnosis and operation. 5 were full term infants (FTIs); age of referral 4 hours -2.5 years. Associated cardiac anomalies in 3 FTIs were more complex than those associated with prematurity. Of the 5 FTIs 2 had surgical procedures aged 3 to 7 months. 3 died less than 1 month after diagnosis or operation. 3 of 8 had stenosis of all 4 PVs (1 patient was premature). They tended to be referred earlier than those with 1 or 2 PVS but not significantly. In our study 5 of 8 patients died at a range of 0.75 to $1 . \overline{3}$ months after diagnosis or operation, 3 survived but are severely symptomatic

The diagnosis depends on a high index of suspicion. Crosssectional echocardiographic examination of all PVs should be mandatory inpatients with pulmonary hypertension, particularly those with a structurally normal heart. Both the number of PVs involved and severity of stenosis influence outcome, which is poor. Our study, indicates an association with prematurity.

\section{P173}

Cardiac surgery during infancy and adolescence: impact on long-term psychological and social development J.S. Sachweh, J. Rixen, B.J. Messmer, S.H. Daebritz

L.ML München, Klinikum Grosshadem, Dept. of Cardiac Surgery, Munich, $D$

Objective: Only little is known about the psychological and social development of children after open heart surgery. The aim of this study was to evaluate the psvchological effects of heart disease and heart surgery in the long-term course after cardiac surgery during infancy and adolescence.

Methods: Between 1978 and 1981, 63 patients $(19 \%<1$ year, $35 \%<6$ years, $19 \%<12$ years, $27 \%>12$ years) were operated on for correction of different congenital heart disease. At the age of 16-42 years, these 29 male and 34 female patients were classified according to the underlying malformation and to their ability index (Somerville und Warnes, 1992). The patients and their physicians were sent questionnaires with 86 questions concerning school/profession, family life, social integration, personality traits and physical situation.

Results: Almost all patients underwent a regular school education. Most patients were satisfied with their job. Family bounding was high; $40 \%$ considered themselves as overprotected, in $40 \%$ the mother quit her job due to the cardiac disease of the child. The majority had fixed pear groups and lived in stable relationships; 18 had children (four with cardiac malformations). In childhood, physical restrictions, in adolescence and adulthood medical and professional worries were regarded as limiting quality of life.
The severity of malformation influenced the result in 19/67 fields of questions significantly (profession, independence, physical limitations), a decreased ability index in 38/67 (profession, family life, social/physical situation, personality traits).

Conclusions: The majority of patients live a normal life Psychological development in long-term follow-up after cardiac surgery in infancy is closely related to physical limitations. Selfassessment of the patients is depending on the social environment. Social acceptance, medical information and individual support help to feel 'normal.

\section{P174}

The investigation of the natural course of interatrial communications diagnosed in 585 newborns O. Bostan, E. Cil

Cludag Lniversity, Medical Faculty, Pediatric Cardiology, Bursa, TR

The aim of this study was to evaluate the rate of spontaneous closure of interatrial communications (IAC), to determine the relationship between the closure and the size and other factors. Between January 2000 and October 2001, a total of 700 asymptomatic and term newborn infants were evaluated. Those infants who had other congenital heart diseases and did not attend on the follow-up were excluded from the study. The remaining 585 newborn infants were followed up for 10.67.1 months (range 1 to 21). The mean age at the diagnosis was 1.71 .4 days (range 1 to 7 ). All of the cases had a detailed examination and in addition electrocardiographic and echocardiographic evaluation. The newborn infants were classified into four groups based on the initial size of IAC. The follow-up controls were performed on the 1st month, 3rd month and on every 3 months for a maximum time of 21 months.

In a period of 21 months, IAC were closed spontaneously in $98.1 \%$ of the cases. In the IAC, which were smaller than $3 \mathrm{~mm}$, the incidence of spontaneous closure was $100 \%$. There was significant correlation between diameter of the IAC and timing of the closure $(p<0.01)$. The closure time in the cases with septal aneurysm is significantly longer than the cases with valve like opening and multiple fenestrations. In the girls, the defects remained open significantly longer than the boys $(p<0.05)$. There was no significant correlation between mitral valve prolapse and interatrial septal aneurysms and also atrial arrhythmias $(\mathrm{p}>0.05)$.

In conclusion, these results suggest that the cases with IAC smaller than $3 \mathrm{~mm}$ may not need the follow-up. Those with atrial septal aneurysm and multiple fenestrations should be followed up because these defects can remain open. Spontaneous closure occurs significantly earlier in cases whose IAC were valve-like opening. We think that these results are important to determine natural course of IAC and to inform the parents about the future of the defect

\section{P175}

The use of angiotensine-converting enzyme inhibitors in the newborn. A $5 \frac{1}{2}$ year experience

S. Ferreira, G. Pereira, A.J. Macedo, S. Kaku

Servio Cardiologia Peditrica Hospital Santa Marta, Lisbon, $P$

Although the use of angiotensine-converting enzyme inhibitors (ACEI) in newborn with congestive heart failure has been suggested, small experience with these drugs in this age group and fear of it's secondary effects, have probably restricted their prescription. The aims were to evaluate the effects of ACEI's use in the newborns admitted to a Pediatric Cardiology Center, from January 1995 to June 2001. Retrospective analysis of the data of 23 newborn treated $(5,6 \%$ of all newborns). Median age was 
9 days and 13 were male $(56 \%)$. Fifteen had a gestational age of 37 weeks or more, 35 weeks being the lowest age. Birth weight ranged from 2000 to $4030 \mathrm{gr}$. Median weight on admission was $3010 \mathrm{gr}$. All had cardiac disease, namely left-to-right shunt lesions, cyanotic heart disease and left heart obstructive lesions. Heart failure and or systemic hypertension were the main clinical manifestations. Median age on the first day of therapy was 18 days. Thirteen received Captopril (initial dosage: $0,6 \mathrm{mg} / \mathrm{Kg} / \mathrm{d}$; maximum: $1,4 \mathrm{mg} / \mathrm{Kg} /$ day $)$ and ten received Enalapril $(0,18 \mathrm{mg} / \mathrm{Kg} / \mathrm{d}$; $0,28 \mathrm{mg} / \mathrm{Kg} /$ day). Median duration of treatment was 21 days with Captopril and 6,5 days with Enalapril. All patients were also treated with diuretics and in some digitalis was added. Evaluations of blood pressure, blood count, kidney function and serum electrolytes, before and after the administration of ACEI were performed.Treatment was suspended in a newborn treated with Enalapril. In 3, dosage was reduced (2 on Enalapril and one on Captopril), due to hypotension. Impaired renal function was recorded in 1 newborn, but no serious electrolyte abnormalities were noticed. There were 5 deaths: 3 due to surgical complications and 2 because of severe septic shock. Thirteen of the 18 -surviving patients had good results with ACEI. We conclude that in this group of newborns, good results were achieved, with improvement of congestive heart failure and with no serious side effects. Although the number of patients does not allow drawing sound conclusions, we could notice better tolerance with Captopril than with Enalapril, especially concerning hypotension.

\section{P176}

\section{Splenic function and immune status in patients with} atrial isomerism

B.H.P. Nagel, J. Paul, J. North, M. Drayson, T. Robertson, O. Stumper University Children's Hospital, Essen, D

To determine functional and anatomical splenic status and B cell function in survivors with left (LAI) and right atrial isomerism (RAI). The practice of penicillin prophylaxis (PP) and pneumococcal vaccination (PV), and the parental knowledge of splenic status was assessed.

In 37 patients with atrial isomerism (LAI: $\mathrm{n}=22$; age 6.8 (1.7-15.1) years; RAI: $n=15$; age $7.5(3.2-15.9)$ years) abdominal ultrasound (US), blood films for Howell Jolly Bodies (HJB) and tests for functional pneumococcal antibodies (PAB) were performed.

US: Asplenia was documented in 12 patients (LAI $\mathrm{n}=1(5 \%)$ vs RAI $\mathrm{n}=11(73 \%))$, polysplenia in $16(\mathrm{n}=15(71 \%)$ vs. $\mathrm{n}=1$ $(7 \%))$, and single spleen in $8(n=5(25 \%)$ vs. $n=3(20 \%))$. One patient could not be scanned. HJB: These were found in $11 / 12$ patients with asplenia, $2 / 16$ with polysplenia, $1 / 8$ with single spleen, and in the one patient without US. In 12 patients blood films could be compared with postnatal films. 3 positive postnatal films for $H J B$ turned into negative (polysplenia $n=2$, single spleen $n=1$ ), 5 negative turned into positive (polysplenia $n=2$, asplenia $n=1$, single spleen $n=1$, no US $n=1$ ). None of the latter had PP or PV. PAB: Of the 15 patients with HJB 5 had low $(<\mathrm{P} 3)$ total $\operatorname{IgG}$ and $\operatorname{IgG} 2$ levels (all on $\mathrm{PP}, 2$ without $\mathrm{PV}$ and 3 up to 2 years after PV). Of those without HJB $(N=22) 6$ had abnormally low PAB, 3 with low IgG and IgG2 and 3 with low IgG2 only. None had PV, only 1 patient had PP. Parental knowledge: Of 28 patients with abnormal splenic status on US only 13 parents knew about the splenic abnormality and the risk of infection.

Conclusions: HJB in patients with single spleen and polysplenia are indicative for functional hyposplenia. The presence of $\mathrm{HJB}$ can change with age. Regular blood films should be obtained. Low PAB (IgG total or IgG2) in patients without HJB may indicate patients at risk for sepsis (PV recommended). PAB should therefore be assessed in all patients. Response to PV is often insufficient. Parental knowledge of splenic status and potential risk of infection and thus compliance for PP and PV is unsatisfactory.

\section{P177}

Coagulation and fibrinolysis profile in children with complex cardiac defect undergoing cardiac surgery R. Heying, S. Wilhelm, K. Schumacher, R. Grabitz, W. van Oeveren, B.J. Messmer, G. von Bernuth, M.-C. Seghaye Children's Hospital Dusseldorf, Dusseldorf, D

Background: Cardiac surgery leads to the production of inflammatory mediators which also have pro-coagulant activity and could therefore be responsible for postoperative coagulation and fibrinolysis disorders.

Objective: To investigate the effect of cardiac surgery on coagulation and fibrinolysis in children undergoing cardiac surgery for complex cardiac defect and to assess the influence of the univentricular palliation on these findings.

Methods: 18 children (age: 3-163 months) undergoing total cavopulmonary connection (TCPC) $(\mathrm{n}=10)$ or biventricular repair $(n=8)$ were enrolled. Coagulation and fibrinolysis were evaluated by measuring plasma levels of prothrombin fragment $1+2(\mathrm{~F} 1+2)$, thromboxane $\mathrm{B} 2(\mathrm{TBX})$, tissue factor pathway inhibitor (TFPI) and monocyte chemoattractant protein-1 (MCP-1) before, during and after cardiac surgery.

Results: In all patients, cardiac surgery was associated with a significant but transitory increase of $F 1+2$, of TBX, TFPI and MCP-1. Maximal values of F1 $+2, T B X$ and MCP-1 were found at the end of bypass while those of TFPI were observed at the beginning of bypass. Markers of coagulation and fibrinolysis returned to preoperative values from the first postoperative day on. Early postoperative TFPI levels were significantly lower and TBX levels significant higher in patients with TCPC than in those with biventricular repair.

Thromboembolic events were not observed in this series.

Conclusion: Our data demonstrate that children undergoing cardiac surgery have profound but transitory alterations of the coagulation and fibrinolysis which could lead to thromboembolic complications. These alterations are more important after univentricular palliation than biventricular repair for complex malformations.

\section{P178}

Long term outcome after correction for congenital vascular compression of trachea and main bronchi M. Buss, E. Mueller, T. Nicolai, C. Doehlemann, G. Balling, H. Netz Depr. of Ped. Cardiology Grohadern, University Munich, Minchen, $D$

We examined 21 patients aged 9-19 years, who had suffered from a significant tracheal or bronchial obstruction by vascular compression in infancy.

Cause of the obstruction was a double aortic arch $(\mathrm{n}=1)$, right descending aortic arch with ductal ligament $(n=1)$, pulmonary sling $(n=3)$ and truncus brachiocephalicus $(n=16) .10$ patients suffered an acute life threatening event and 16 needed mechanical ventilation preoperatively. Corrective surgery took place during infancy, in two cases in the third year of life. On presentation a thorough clinical examination was followed by ECG, echocardiography, X-ray of the thorax, lung function and exercise testing with monitoring of blood pressure, oxygen saturation and capillary blood gas analysis. At the time of examination 10 patients complained of exercise related symptoms. 12 patients had scelettal or muscular anomalies of the thorax. 
Cardiac function as measured by echo was regular in all patients. 8 patiens had abnormal lung function with limited peak expiratory How, 4 showed a variable degree of restriction. No obstructive dysfunction was observed. 8 patients had a limited exercise capacity, 6 showed an in- or expiratory stridor on exercising. 3 patients needed treatment for skoliosis.

Despite severe and in some cases life threatening symptoms in infancy only 4 patients demonstrated exercise related symptoms on examination. In conclusion even severe stenosis of trachea and main brochi by vascular structures has a good prognosis when surgical decompression is carried out early in life.

\section{P179}

Value and limitations of 3 different criteria for the diagnosis of infective endocarditis (IE) in children

P. Tissires, A. Gervaix, M. Beghetti, E. Jaeggi

Pediatric Cardiology Unit, University Children's Hospital, Geneva, CH

Objective: To compare the sensitivity in diagnosing IE by 3 different criteria: 1) von Reyn (VR; Ann Int Med 1981), 2) Duke (DC; Am J Med 1994), and 3) modified Duke (MD; Clin Infect Dis 2000).

Methods and Results: The 3 classifications were applied in 39 consecutive children $(7.6+5.1$ years) diagnosed with IE between 1985 and 2001. IE diagnosis was based on echocardiographic and microbiological/pathological exams. Initial echocardiography suggested IE in 95\% (33 vegetations; 4 new valvar leaks). Blood cultures or specimen bacteriological exams were positive in $74 \%$. PCR or serology were essential in 5\%. Most culture negative cases had prior antibiotic therapy. Sensitivities for the diagnosis of IE were $38.5 \%, 76.9 \%$ and $84.6 \%$ for von Rein, Duke and modified Duke criteria. In 20.5\% IE was misclassified as rejected by VR, but was definite or possible by DC and MD. In 3 cases the diagnosis of IE was possible by DC but definite by $\mathrm{MD}: 2 / 3$ had 1 major and $>3$ minor symptoms and $1 / 3$ had $Q$-fever.

Conclusion: MD has a higher sensitivity than VR and DC for diagnosing IE in children. However, still 15\% remained classified as possible IE in our series.

\section{P180}

Delayed sternal closure in neonatal cardiac surgery; additional risk for postoperative intensive care? I. Daehnert, J. Bratanow, J. Theile, J. Hambsch, A. Moeckel, M. Kostelka Klinik fuer Kinderkardiologie, Herzzentrum Leipzig, Leipzig, D

Objective: Delayed sternal closure is a widely accepted procedure after complex cardiac surgery in neonates, but often also regarded as complication and significant risk. Aim of this study is the comparison of the postoperative course of neonates with or without delayed sternal closure to identify possible risk factors.

Methods: Retrospective analysis of 114 consecutive operations on newborns in one institution by one surgeon from $8 / 98$ to $8 / 01$. Results: There were 39 operations without cardiac bypass (group A). From 75 procedures with cardiac bypass, 26 had immediate sternal closure (group B) and 49 delayed sternal closure (group C). At admission on the pediatric intensive care unit the groups varied significantly in diagnoses, duration of operation, bypass time, lactat level and dosis of inotropic support. In hospital mortality was $11 / 114(10 \%)$ in total with $1 / 39(2,6 \%)$ in $\mathrm{A}, 0 / 26$ in $\mathrm{B}$ and $10 / 49(20 \%)$ in C, resp. Group C contained 15 patients with hypoplastic left heart, $5(33 \%)$ of them died. Delayed sternal closure was performed in 39 patients on day 1 to 17 (median 2) after surgery. Median intubation time was 2 days $(0-23)$ in $A, 3$ days $(1-16)$ in $B$ and 6 days (1-28) in C, resp. There were no significant differences in the rates of infection, coagulation disorder, renal insufficiency, nitric oxid requirement and renal failure between the groups $\mathrm{B}$ and $\mathrm{C}$.

Conclusion: Newborns requiring cardiac surgery are at high risk. Delayed sternal closure is applied only in critically ill patients or after the most complex procedures. It is not an independent additional risk factor for in hospital death or major complications.

\section{P181}

Succesful interferon Alfa-2A therapy for severe cyanosis secondary to pulmonary diffuse hemangiomatosis C. Michel, C. Barrea, C. Ovaer, M. Cailteux, C. Debauche, T. Sluysmans Cliniques Lniversitaires Saint-Luc, Bruxelles, B

Introduction: Pulmonary hemangiomatosis is a rare proliferative pulmonary process which affects children and voung adults. This microvascular disorder progressively leads to pulmonary hypertension, cyanosis, bleeding, congestive heart failure and progress rapidly, causing death in most cases within one to four years.

Case report: In a one-year-old girl presenting with severe cyanosis (aortic $\mathrm{O}_{2}$ sat: $80-85 \%$ ), we diagnosed a diffuse pulmonary hemangiomatosis with intrapulmonary right-to-left microshunts. The shunt was predominent in the right lung on pulmonary angiography and pulmonary scintigraphy. The right-to-left shunt was calculated at $30 \%$ by scintigraphy and oxymetry $\left(\mathrm{O}_{2}\right.$ sat: right pulm. veins $64 \%$ and $83 \%$, left pulm. veins $96 \%$ and $99 \%$ ). Based on the potential inhibition of endothelial proliferation by interferon in heterogeneous localisation of hemangiomatosis, and a beneficial effect of interferon therapy in one case of pulmonary hemangiomatosis reported in the literature, interferon Alfa-2A therapy ( 1 to 3 million U/m2 BSA subcutaneously per day) was started for a period of 4 months. No complication occured and a beneficial effect was observed: aortic $\mathrm{O}_{2}$ sat increased from $80 \%$ to $96 \%$ At 4 years follow-up, the child evolution is excellent and aortic $\mathrm{O}_{2}$ sat remains above $95 \%$.

In conclusion: Pulmonary hemangiomatosis is usually a fatal disease. Its diagnosis requires a high index of suspicion and an aggressive therapeutic approach. We observed a beneficial and long term effect of Interferon Alfa-2A in this rare and severe pulmonary disorder.

\section{P182}

Long-term time-domain heart rate variability in patients with Rett syndrome

T. Karagoz, M. Cinbis, A. Celiker, M. Topcu

Hacettepe University Faculty of Medicine, Ankara, TR

Although patients with Rett syndrome may survive into middle and old age, their life expactancy is reduced and the incidence of sudden death is greater than that of the general population. Heart rate variability indicating either increased sympathetic activity or reduced baroreflex sensitivity has been shown to be strongly predictive for sudden cardiac death in the general population and in patients with myocardial infarction and some other diseases. Corrected QT interval prolongations, $\mathrm{T}$-wave abnormalities and decreased frequency-domain short-term heart rate variability were demonstrated in a few studies and suggested to be as a possible explanation for sudden death. The goals of this study was to examine time-domain long-term heart rate variability indices (RMSSD, pNN50, HRV triangular index) which provide an index of parasempatethic function on 24-hour ECG recordings in 25 patients (mean age $7.03 .8 \mathrm{yr}$ ) with Rett syndrome and to correlate this to electrocardiographic and echocardiographic variables and compare the results with 23 similar aged healthy controls (mean age $7.64 .1 \mathrm{yr}$ ). Heart rate variability was measured by standard 
time-domain techniques (Rozinn Electronics, Inc., New York). Electrocardiographic indices including QRS duration, QT, QTc and QT dispersion were measured by an computerized ECG analyzer (Cardio Control N.V.). 2-D, M-mode and Doppler echocardiographic study including left ventricular systolic and diastolic functions were performed by Vingmed System Five. All RMSSD, pNN50 and HRV triangular index were reduced in patients $(5731 \mathrm{~ms}, 16.612 .5 \%, 28.19 .2 \mathrm{~ms})$ when compared with controls $(9247 \mathrm{~ms}, 32.321 .3 \%, 3715.5 \mathrm{~ms})$. Athough we did not find significant difference between the two groups with respect to QTc, other electrocardiographic and echocardiographic indices, the QTc was greater than $440 \mathrm{~ms}$ in four of the patients. Reduced heart rate variability indices were correlated with increased $Q T c$ (RMSSD-QT $\mathrm{c}: \mathrm{r}=-0.443, \mathrm{p}=0.030 ; \mathrm{pNN50-QTc:} \mathrm{r}=-422$, $\mathrm{p}=0.040 ; \mathrm{HRV}$ triangular index: $\mathrm{r}=-0.441, \mathrm{p}=0.045)$. Reduced heart rate variability was also related with increased heart rate and decreased total diastolic filling. In conclusion, time-domain HRV indices assessed from 24-hour ECG monitoring showed decrease in parasympathetic tone in patients with Rett syndrome, which was related to increased QTc and suggest a possible cardiac basis for sudden death.

\section{P183}

Oxidized low density lipoprotein in patients with Kawasaki disease

Y.C. Fu, B. Hwang, S.L.Jan, C.H. Kao, C.S. Chi

Taichung Veterans General Hospital, Taichung, TAI

Background: Recently, more and more studies have demonstrated that oxidized low density lipoprotein (ox-LDL) played a key role in the pathogenesis of adult coronary artery disease. This study was to investigate the ox-LDL and general lipoprotein profiles in patients with Kawasaki disease.

Methods: Forty consecutive patients with Kawasaki disease who underwent cardiac catheterization were studied prospectively. We classified the patients into two groups: Group A consisted of 12 patients with coronary artery disease, coronary stenosis or aneurysm. Group B consisted of 28 patients with normal coronary angiography. Blood sample was tested for the levels of ox-LDL autoantibody, total cholesterol, high-density lipoprotein cholesterol (HDL-C), low-density lipoprotein cholesterol (LDL-C), and triglycerides (TG).

Results: There were no significant differences between two groups in gender, age of onset of disease, TG, cholesterol, HDL-C, LDL-C, and TC/HDL. There was significantly higher level of ox-LDL in group A than that in group B ( $\mathrm{p}=0.008)$.

Conclusions: There is significantly higher level of ox-LDL in patients of Kawasaki disease with coronary artery disease. The role of ox-LDL needs further studies.

\section{P184}

Aortic dissection in children and young adults; Canadian/Israeli experience

E. Zalzstein, R. Hamilton, S. Diamant, G. Webb

Soroka Medical Center, Pediatric Cardiology, Beer Sheva, IL

Background: Aortic dissection in the pediatric age group is rare. The main challenge in managing acute aortic dissection is to suspect and thus diagnose the disease as early as possible.

Objective: The aim of this study is to heighten the awareness for this potentially dangerous medical diagnosis.

Methods and Results: We reviewed retrospectively the charts of all patients age $0-21$ years who were diagnosed during the period of 1970-2000 as having Aortic dissection in 4 medical centers.
(Toronto - 2, Beer Sheva - 1, Tel Aviv - 1). 13 patients were identified. There were 7 male patients and 6 females. Mean age at diagnosis was 12.3 years (age range: 5 days-21 years). In 12 patients the dissection was acute and in one chronic. Congenital heart defects were present in 5 patients, Marfan syndrome in 4 patients. In 3 patients aortic dissection developed as a complication during medical procedures (in 2 - cardiac catheterization, and 1 - cannulation during cardiac surgery). In 3 patients - following blunt trauma to the chest during road accident. Presenting symptoms were: chest pain - in 4 patients, abdominal pain and signs of ischemic bowel -2 , non palpable femoral pulses in 1, SVC obstruction in 1 . The main diagnostic tool was aortic angiography and MRI. Outcome: overall mortality was $38 \%$, and only 6 patients underwent successful surgical procedures.

Conclusion: Due to the high mortality rate and as a consequence of its rarity, high index of suspicion should be raised in any young patient with chest pain who is known to have Marfan syndrome, aortic valve and arch anomalies, and blunt trauma to the chest Careful physical examination and use of new diagnostic tools may reduce the mortality.

\section{P185}

Patients with aortic coarctation operated during the first year of life, different surgical techniques and prognostic factors; experience of 20 years

A. Carrio, C. Moura, J. Monterroso, R. Sonsa, J. Casanova, P. Bastos, J.C. Areias

Pediatric Cardiology Service, Hospital S. Joo, Porto, P

Authors reviewed clinical files of patients with Coarctation of the Aorta (CoAo) operated during the first year of life, in order to access the outcome of different surgical techniques.

Material and methods: The study included patients operated since 1st June 1980 till 1st June 2001. Patients were divided in three groups according to the diagnosis: Group 1-isolated CoAo (with or without Persistent Ductus Arteriosus (PDA), group 2-CoAo plus Ventricular Septal Defect (VSD), group 3-CoAo plus complex heart defect. Surgical techniques were divided in four groups: Group A-resection of the CoAo with a end-to-end anastomosis. group B-Waldhausen (subclavian flap), group C-enlargement of the aorta with a patch, group D-Waldhausen and resection of the CoAo with a end-to-end anastomosis. Parameters analysed: sex, age at surgery, other surgical procedures during surgery, re-operation and outcome.

Results: 156 patients were operated of whom $84,5 \%$ since 1989 , $61,5 \%$ were male. Surgery was performed between 3 and 336 days of life (average-154). 60,9\% patients were included in group 1 . $32,7 \%$ in group 2 and $6,4 \%$ in group $3.25 \%$ patients had a group A surgery, $60,2 \%$ had a group $B, 7,0 \%$ had a group $C$ and $7,7 \%$ had a group D surgery. PDA was sectioned in $48 \%$ patients. Banding of the pulmonary artery was performed in $19,9 \%$ cases during the first surgery. $18,6 \%$ needed a second surgery. Total mortality was $19,2 \%$ from which $56,7 \%$ died during the period they were admitted for surgery. $53.3 \%$ died between 1989 and 1995 and $36.6 \%$ after 1995 . The mortality per groups was: Group $1-15,8 \%$, group $2-21,5 \%$, group $3-30 \%$, group A-18\%, group B-19,1\%, group C$27,2 \%$ and group D-16,7\%. In $11,5 \%$ of the patients a Re-CoAo was found during follow-up, $16,6 \%$ of which had surgical repair. $5,1 \%$ were submitted to balloon angioplasty and $3,9 \%$ were considered to have no indication for treatment. $40 \%$ of the patients are doing well. $29,4 \%$ were lost for follow-up.

Conclusions: Total mortality has been decreasing. We found the best results when the CoAo appears isolated and the highest mortality was found in patients with associated complex heart defects. 
1 surgical techniques showed similar results except group C. sng-term results were generally good with few Re-CoAo and od quality of life.

186

lidated method to assess quality of life in vildren and adolescents with heart disease Birkeland, A. Rydberg

ept. of Clinical Sciences, Paediatrics, Sect. of Paed.Cardiol., Lme, S

diatric cardiology has undergone extensive development given sult in a new group of surviving patients with complex congeniI heart disease and a complex psychosocial situation. Along with edical development, there is a need for specific, validated psylosocial instruments to measure and describe the psychosocial mplexity and quality of life (QoL).

udy group: Ninety-seven patients, 47 boys and 50 girls were aded into three categories (I-III) with respect to complexity of sngenital heart disease (CHI)). Group I comprised 42 patients, oup Il 20 patients and group III 35 patients. An age- and sexatched control group consisting of 73 healthy children/youths id the patient group were evaluated with a new inventory ethod for psychosocial symptoms.

iethods: The inventory method is based on a life quality model vided into 3 spheres: personal, interpersonal and external where ch sphere is composed of a part of symptoms. The personal sphere sscribes the childs individual psychological distress symptoms. he interpersonal sphere evaluates symptoms/problems in the mily and in school. The external sphere describes the need for tedical, social and economic support related to the heart disease. he severity of the symptoms is evaluated using a 4 point Lickert ale ranging from 0 to 3 .

esults: The most frequent symptoms in the patient group xcept for somatic symptonss) were anxiety-personal (13/97), mily symptoms-interpersonal $(68 / 97)$ and health care related zeds-external (71/97). In the control group only one child iowed symptoms of anxiety, family symptoms were less frequentterpersonal $(8 / 73)$ and only $11 / 73$ families showed health care lated needs-external.

I the patient group family symptoms constituted the most severe parate interpersonal variable. As expected the group of patients ith the most complex $\mathrm{CHD}$ showed the most common and vere needs of support.

onclusion: This validated method invent psychosocial symptoms patients with complex congenital heart disease and elucidates e psychosocial complexity and aspects of quality of life.

187

valuation of hematologic and hemodynamic arameters in patients with Eisenmenger syndrome Semizel, D. Alchan, A. Grgey

acettepe Lniversity Faculty of Medicine, Ankara, TR

ims: To evaluate the hematologic and hemodynamic parameters children with Eisenmenger sydrome (ES).

iethods: We prospectively studied 23 patients with ES. 20 healthy iildren and 14 patients with congenital heart disease (CHD), 7 anotic and 7 acyanotic, were also studied as control groups. Blood idy for hematologic assesment were performed for all groups. ardiac catheterization records were analysed in patients with ES. esults: In patient with ES, $\mathrm{Hb}, \mathrm{Hct}$ and red blood cell counts (BC) were found to be higher ( $\mathrm{p}$ values were $0.001,0.001$ and 001, respectively), whereas platelet counts were found to be wer $(p=0.001)$ than normal controls. It was also found that prothrombin time activity (PTA) were decreased, prothrombin time (PT), PTINR and activated partial thromboplastin time (aPTT) were increased in patients with ES. In patients with ES, antithrombin III (ATIII) and protein C (PC) activities and free protein S (FPS) levels were also found to be lower ( $\mathrm{p}$ values were $0.001,0.002$ and 0.004 , respectivcly) than in normal controls. Measurenents of bleeding time showed higher values for patients with ES, compared to patients with $\mathrm{CHD}$. For patients with ES, there was a positive correlation between systemic resistance (Rs) and PTA values, a negative correlation between $R s$ and PTINR values, and a positive correlation between Rs and ATIII activities. A positive correlation between pulmonary-to-systemic resistance ratio ( $\mathrm{Rp} / \mathrm{Rs}$ ) and Hct $(p=0.018, r=0.489), \operatorname{RBC}(p=0.029, r=0.456)$, aPTT $(p=0.011, r=0.534)$, trombin time $(p=0.013, r=0.537)$, and $a$ negative correlation between right atrial pressure (RAP) and PTA $(p=0.005, r=-0.574)$, and a positive correlation between RAP and PT $(p=0.002, r=0.627)$ and PTINR $(p=0.006, r=0.571)$ were also found in patient with ES

Conclusion: Polycythemia is seen in patients with ES, and the severity of polycythemia is directly related to the degree of $\mathrm{Rp}$. There is also a tendency towards bleeding in patients with ES and this is also directly related with Rp. But for evaluation of bleeding tendency and possible consumption coagulopathy, which can be seen in patients with ES, each child must be assessed individually.

\section{P188}

Need for closure of isolated secundum atrial septal defect (ASD II) in infancy

A. Lammers, A. Eicken, M. Hauser, R. Lange*, J. Hess

Dept. of Pediatric Cardiology and Congenital Heart Disease, Munich, $D$

Introduction: Isolated ASD II in asymptomatic children is generally closed at the age of $\overline{5}-6$ either by catheter or surgery.

Rarely closure at a younger age is considered because of symptoms like failure to thrive, pulmonary hypertension or cardiac failure even in infancy

Purpose: This study was initiated to assess which conditions led to the decision of early closure and whether this resulted in a better performance and growth of these children.

Patients: Since 1.1.1990, 23 infants ( $\mathrm{f}=17, \mathrm{~m}=7$ ) underwent surgical closure of an ASD Il at a mean age of 6.7 months (range: 1.3-11.6 months). 10 infants were ex-prematures, of whom 7 had bronchopulmonary dysplasia, 3 after longstanding mechanical ventilation preoperatively. In total 12 patients had defined syndromes. All infants were symptomatic (tachydyspnoea, failure to thrive). All patients except one had preoperative invasive hemodynamical evaluation.

Results: Mean PAP was $48 \mathrm{mmHg}$ (range: $19-91 \mathrm{mmHg}$ ), in 16 patients there was pulmonary hypertension, defined as PAPsyst. $>30 \mathrm{mmHg}$. In 10 patients pulmonary vascular resistence was greater than 3 WUxm. Mean Qp/Qs-ratio was 3.6:1 (range: $1.07: 1 \rightarrow 10: 1)$.

The follow-up time was 48.5 months (range: $4-123$ months) There was no early mortality. One patient died years after surgery from persisting pulmonary hypertension.

In $1+(88 \%)$ children the PAP proved to be normal at follow-up. In nearly all patients growth and development returned to normal except in those in whom syndrom associated retardation was present.

Conclusion: If lungs are compromised, either by mechanical compression or any preexisting damage of the pulmonary vascular bed, even a minor left to right shunt might be poorly tolerated in infancy. Then early surgical closure of an isolated ASD II is indicated to support thrive and growth and may prevent the onset of 
irreversible changes of the pulmonary vasculature. Infants with primary pulmonary hypertension and isolated ASD II should be identified and defined as a specific patient group in whom closure of the ASD II should not be performed.

P189 (see Abstract 24)

Quality of life in children with pulmonary atresia and intact ventricular septum (PA-IVS) in Sweden

B-M. Ekmanjoelsson, L. Berntsson, J. Sunnegärdh

The Queen Silvia Childrens Hospital, Cardiology, Skvde, S

P190 (see Abstract 25)

Endocarditis prophylaxis in children with congenital heart disease: parent's awareness

J.L. Barreira, M.J. Baptista, J. Moreira, A.Azevedo, J.C. Areias

Department of Pediatrics, Hospital S. Joo, Porto, P

P191 (see Abstract 48)

Thrombus formation in patients after Fontan operation detected by transoesophageal echocardiography

G. Balling, M. Vogt, H. Kaemmerer, A. Eicken, R. Lange*, J. Hess

Deutsches Herzzentrum München, D

\section{P192}

Mutation analysis of human INV gene in a series of cases with lateralization defect and various congenital heart defects

P. Schn, K. Tsuchiya, D. Lenoir, T. Mochizuki, C. Guichard, S. Takai, A.K. Maiti, J. Weil, T. Yokoyama, P. Botwagnet

Universite Claude Bernard Lyon 1, Lyon, F

Determination of left-right axis is a precocious embryonic event and all phenotypic anomalies resulting from a disruption of the normal lateralization process are collectively referred to as lateralization defect. A transgenic mouse with lateralization defect, hepatic, kidney and pancreatic anomalies resulted from a disruption of the INV gene by insertion of a transgene. The human ortholog gene is thus a good candidate for lateralization defect in humans in particular in cases with associated hepatic anomalies. Here, we identified, mapped and characterized the INV human gene and screened a series of $6 \overline{3}$ heterotaxic patients (with or without biliary anomalies) for mutation in this gene: 19 individuals are born to consanguineous parents, 19 are familial cases. In addition to lateralization defect, 44 have various types of cardiac malformations, 14 have ciliary dyskinesia of the respiratory tracts and 7 have extrahepatic biliary atresia with polysplenia. This later group is phenotypically very similar to the INV transgenic mouse. In a German family of Turkish origin, two children had lateralization defect: a girl presented with situs inversus, ventricular septal defect and L-transposition of the great arteries. Her younger brother had situs inversus, atrial and ventricular septal defects, and partial anomalous pulmonary venous return. Another child deceased from unknown reason. We found that in this family all available affected and unaffected individuals were heterozygous for a mutation in the splicing donor site of intron 12 in the INV gene resulting in 2 different aberrant splicing isoforms. This result could be explained either by randomization of lateralization defects or as suggested earlier, di- or trigenic inheritance although in this family we were unable to detect a mutation in genes already involved in human lateralization defect - (LEFTY1, LEFTY2, ACVR2B, NODAL, ZIC3 and CFC1). By contrast to the mouse, the affected individuals have no biliary anomalies, and absence of mutation in a series of 7 cases with lateralization defect and biliary anomalies demonstrates that INV is not frequently implied in such phenotype in humans.
P193 (see Abstract 22)

Genetic transmission of vagal hyperreactivity in rabbits

A. Livolsi, J. Feldman, J. Feingold, Y. Alembik, L. Weiss,

M. Fischbach, J. Messer, P. Bousquet

Service de Pdiatrie 1-Chu de Hautepierre, Strasbourg, $F$

\section{P194}

Ventricular dyssynchrony affects biventricular function

late after repair of tetralogy of Fallot

J. Janousek, R.A. Gebauer, O. Reich, J. Marek, P. Vojtovic

Kardiocentrum, Univ. Hosp. Motol, Prague, CZR

Influence of right bundle branch block (RBBB) induced ventricular dyssynchrony on biventricular function was studied in 184 long-term survivors 7.95 .4 years after surgical correction of tetralogy of Fallot. Systolic and diastolic right ventricular (RV, functional part only) and left ventricular (LV) function was assessed by radionuclide ventriculography. A total of 241 radionuclide data sets ( 57 patients had 2 evaluations) were correlated with concurrent QRS duration, aortic cross-clamp time, presence of transannular patch, age at surgery, interval from surgery and grade of pulmonary regurgitation assessed by echocardiography.

Results: QRS duration (mean $14826 \mathrm{~ms}, \mathrm{RBBB}$ in all) did not differ between patients with absent, moderate or severe pulmonary regurgitation $(14528,14824$ and $15027 \mathrm{~ms}$, resp.). QRS duration correlated negatively with $R V$ ejection fraction (RVEF, $R=0.259$, $\mathrm{p}<0.001$ ), RV peak ejection tate (RVPER, $\mathrm{R}=0.320$, $\mathrm{p}<0.001)$, $\mathrm{LV}$ peak ejection rate $(\mathrm{LVPER}, \mathrm{R}=0.307, \mathrm{p}<0.001)$, $\mathrm{LV}$ peak filling rate (LVPFR, $\mathrm{R}=0.374, \mathrm{p}<0.001)$ and positively with $R V$ preejection period (RVPEP, $R=0.350, p<0.001$ ) and interval between $R V$ and $L V$ enddiastole and peak ejection rate $(\mathrm{R}=0.416, \mathrm{p}<0.001$ and $\mathrm{R}=0.234, \mathrm{p}<0.001$, resp.). In a multivariate analysis, QRS duration was the strongest independent negative predictor of RVEF, RVPER, LVPFR and positive predictor of RVPEP and interval from RV enddiastole to peak ejection rate $(\mathrm{p}<0.001$ for all), whereas grade of pulmonary regurgitation reached significance only for RVPER ( $p=0.002)$. Subanalysis confirmed similar influence of QRS duration in the subgroups without. with moderate and with severe pulmonary regurgitation, resp. Conclusions: RBBB affects negatively right and left ventricular function parameters presumably due to dyssynchronous ventricular activation and ventricular contractile discoordination. This effect is independent of right ventricular volume overload and contributes to impaired ventricular function late after repair of tetralogy of Fallot.

\section{P195}

Cardiac output, pulmonary and systemic pressures up to maximal exercise in patients operated long time ago for VSD with increased pulmonary pressure

B.O. Eriksson, D. Holmgren, G. Sdow

Departments of Pediatric Cardiology, Göteborg, $S$

Increased pulmonary pressures is an essential indication for surgery of VSD. Nowadays this operation is performed before 6 months of age to avoid pulmonary hypertension. However, before surgery was possible there were patients, who had lived long time with their increased pulmonary pressures. It is under debate whether this situation might have caused any sequaele later on in life. We therefore wanted to study the hemodynamic regulation at rest and during exercise in such a group of patients. Material: We selected those patients operated at our institution who had had a preoperative systolic pulmonary pressure $>49 \mathrm{mmHg}$ and where long time had elapsed since their operation. Out of 
19 patients fulfilling the criterias 12 volunteered to this study, 7 women and 5 men. They were as a mean 21.1 years and 14.2 years had elapsed since their operations.

Methods: All performed exercise tests up to maximal level to achieve their aerobic power. In the hemodynamic study two to three submaximal loads were used before the maximal load. Cardiac output was measured with the dye dilution method. Oxygen uptake was achieved with the Douglas bag technique and gas analyses with the micro-Schollander method. Catheters were placed in the pulmonary artery and in a brachial artery to get simultaneous pressure recordings during exercise

Results: Three of the patiens reached max. $\mathrm{VO}_{2}$ at the study. As a mean the patients highest load represented $85.3 \%$ of their maximal $\mathrm{VO}_{2}$. Cardiac output at the maximal load was as a mean $12.1 \mathrm{l} / \mathrm{min}$, stroke volume $67.5 \mathrm{ml}$ and $\mathrm{aVO}_{2}$-diff. $14.0 \mathrm{ml} / 100 \mathrm{ml}$. The pulmonary pressures were $34.5 / 11.0, \mathrm{~m}=20.3 \mathrm{mmHg}$ at rest and increased to $66.7 / 24.1, \mathrm{~m}=41.5$ at maximal exercise. In two men their pulmonary artery pressures exceeded their systemic pressures. They did also fall in cardiac output and stroke volume at their highest load as an indication of right ventricular failure. Summary: Long-term after surgery patients with VSD and increased pulmonary pressures preoperatively show marked alteration in their hemodynamic response to exercise.

\section{P196}

\section{Left atrial appendage obliteration in piglets}

E. B. Sideris, S. Toumanides, M. Petrou, S.D. Moulopoulos

Athenian Institute of Pediatric Cardiology, Athens, GR

The left atrial appendage (LAA) is the most common site of thrombus formation in adults with atrial fibrillation. Its obliteration could prevent thromboembolic cerebrovascular episodes. Covered stenting rings (CSRs) were introduced in the LAA in 6 piglets, $10-12 \mathrm{Kg}$. The covered stenting ring is a polyurethane foam covered nitinol device, collapsible in the introducing sheath and automatically re-expandable in the LAA. The device is retractable in the introducing sheath prior to release. CSRs can automatically expand in diameters $3-25 \mathrm{~mm}$ and accomodate LAAs of different sizes and shapes; they require $11 \mathrm{~F}$ introduction. The method involves transvenous introduction of the device, through a Mullins type sheath into the LAA, through the foramen ovale. Transeptal puncture can be performed in case of closed foramen ovale. Left atrial angiography was performed before, right after and a week after device implantation. Pathology was performed a week after implantation in 5 animals and two months later in one. All CSDs were released in LAAs without any incidents of embolization or other complication. The immediate post implantation angiogram demonstrated only partial LAA obliteration; however full LAA obliteration was noticed a week later. Pathology confirmed the occlusion of the LAA mouth and LAA appentage obliteration. In conclusion CSRs can obliterate the LAA in piglets. The device is easily applicable and effective. The safery of the method needs to be established by further testing, prior to human use.

\section{P197}

\section{Peripheral vascular resistance and cardiac output in} children after aortic coarctation repair

U. Giordano, G. Crosio, L. Di Pangrazio, S. Giannico, A. Turchetta,

A. Calzolari

Bambino Ges Children's Hospital, Rome, I

Long term follow-up studies still show a limited life expectancy in patients $(\mathrm{P})$ following repair for aortic coarctation (AC); in particular, hypertension is a frequent complication in these $\mathrm{P}$.
Aim: To assess, at rest (r) and at peak (p) exercise, the blood pressure $(\mathrm{BP})$ behavior and the cardiac output $(\mathrm{CO})$ to determine the peripheral vascular resistance (PVR) in a group of $20 \mathrm{P}$ after $\mathrm{AC}$ repair (mean age $13.74 .2 \mathrm{yrs} ; 12 \mathrm{~m}-8 \mathrm{f}$ ), not receiving antihypertensive therapy. Treadmill exercise testing (ET) was performed (Bruce prot.) and $\mathrm{CO}$ determined (acetylene rebreathing method) at $\mathrm{r}$ and at $\mathrm{p}$ exercise; cardiac index $(\mathrm{CI}=\mathrm{CO} / \mathrm{BSA})$ was also considered. Parameters considered: systolic (S) BP, diastolic (D) $\mathrm{BP}$ and pulse pressure $(\mathrm{PP}=\mathrm{SBP}-\mathrm{DBP}) \mathrm{r}$ and $\mathrm{p}$ in $\mathrm{mmHg}$, time of exercise (TE) in minutes, heart rate changes (difference berween $r$ to $p$ ) (DHR) in bpm, CO $r$ and $p$ in $L / m i n$, mean arterial pressure $(\mathrm{MAP}=\mathrm{SBP}+2 \mathrm{DBP} / 3)$ and the total PVR calculated using the formula: PVR $=\mathrm{MAP} / \mathrm{CO} \times 80 \mathrm{dyne} / \mathrm{cm} / \mathrm{sec}^{-5}$ $\mathrm{P}$ data were compared with those obtained in a group of 20 healthy $(\mathrm{H})$ controls of the same age $(12 .+2.1 \mathrm{yrs} ; \mathrm{p}=\mathrm{n} . \mathrm{s}$; $14 \mathrm{~m}-6 \mathrm{f})$. Student's t test was used and $p$ value was set at $<0.05$; linear regression analysis was used when appropriate.

Data obtained are shown in table. $\mathrm{P}$ when compared with $\mathrm{H}$ showed no significant differences for $\mathrm{CIr}$, a higher PVRr, reduced TE with higher SBPp and reduced increment in DHR. Significant differences were found for SBPr, PP $r$ and $p$, CIp and reduced decrease in PVRp. No differences were found for DBP both $r$ and $p$. Linear regression analysis showed positive relationship between $\mathrm{PPr}$ and $\mathrm{COr}$ only in the $\mathrm{P}$ group $(\mathrm{p}=0.028$; $\mathrm{r}=0.490) . \mathrm{P}$ after $\mathrm{AC}$ repair have an altered peripheral vascular tone associated with an increased $\mathrm{PP}$, both at $\mathrm{r}$ and $\mathrm{p}$ of exercise. These findings could be due to an altered baroreflex control mechanism associated to a progressive increase in resistance during exercise at the site of correction repair due to a local loss of elasticity. Although not overtly hypertensive these $\mathrm{P}$ are at high risk for development of hypertension because of their increased PVR and PP.

\section{P198}

Functionally single ventricle: long term results in adolescents and young adults after Fontan procedure

A. Silvinska, O. Trojnarska, W. Bobkouski, M. Woitalik, B. Mrozinski, A. Szyszka, H. Gorzna-Kaminska, M. Pawelec-Woitalisk,

J. Maciejeuski

Department of Pediatric Cardiology, Poznan, PL

Background: In long-term results in patients (pts) with functionally single ventricle (SV) after Fontan procedure, the improvement of quality of life in the majority of pts is remarkable. Serious complications of treatment are remarkable as well.

Patients and methods: The clinical evaluation of $21 \mathrm{pts}$ with functionally single ventricle was performed $15-19 \mathrm{yrs}$ ( $16.42 .1 \mathrm{yrs})$ after Fontan procedure. The age of pts ranged from $17-21 \mathrm{yrs}$ (18.22.2 yrs) with majority of male $(M, n=14 ; F, n=7)$. The study group consists of 10 pts $(47,6 \%)$ with single inflow to ventricle, 8 pts $(38 \%)$ with double inflow to left ventricle and 3 pts $(14.3 \%)$ with double inflow to right ventricle. The tricuspid atresia was diagnosed in $9 \mathrm{pts}(42.9 \%)$, the mitral atresia in $1 \mathrm{pt}(4.8 \%)$, the transposition of the great vessels in $14 \mathrm{pts}(66,7 \%)$. In $12 \mathrm{pts}$ pulmonary artery banding in infancy, and in 9 pts $(42.9 \%)$ pulmonary-systemic anastomosis were performed. Ages at operation ranged from $3.3-5.5 \mathrm{yrs}(4.10 .7 \mathrm{yrs})$.

Results: The majority of patients (19 pts; $90.5 \%)$ had no any remarkable symptoms, except slight effort intolerance (NYHA I/II). In 2 pts $(9,5 \%)$ exudative protein-losing enteropathy was diagnosed, in 5 pts $(23,8 \%)$ systolic ventricular dysfunction with $E F=40-45 \%(422 \%)$, in 18 pts $(87.7 \%)$ diastolic ventricular dysfunction and in 7 pts (33.3\%) progressing atrioventricular valve insufficiency were diagnosed. In 2 pts $(9.6 \%)$ serious cardiac 
arrhythmia including atrial fibrillation and atrial flutter were observed.

Conclusions: After Fontan procedure the great majority of adolescents and young adults with SV are leading good quality lives, and have no any remarkable complaints, but they have significant disturbances of SV diastolic and systolic function. Serious cardiac arrhythmia occur in about $10 \%$ of them. The progress in surgical techniques and performing operation before occur irreversible myocardial damage may influence improvement of prognosis in Fontan procedure.

\section{P199}

Hypertrofic adaptation of the right ventricle seems important for exercise performance in Mustard patients H. Andersen, J.C. Nilsson, B.A. Groenning, B. Larsson,

J.R. Jacobsen, L. Sondergaard

Dep of Paediatrics, $H$ :S Rigshospitalet, Copenhagen, DK

Background: Previously, transposition of the great arteries (TGA) was treated by atrial switch procedures after which the right ventricle served the systemic circulation. Although patients who have undergone such interventions are generally considered asymptomatic, it is well established that their exercise performance is below normal values. It is uncertain, whether the ability of the right ventricle to adapt to the systemic blood pressure by myocardial hypertrophy influences exercise performance. The aim of the present study was to compare right ventricular myocardial mass (RVM) with maximal oxygen consumption (VO2max) in patients with TGA corrected by the Mustard procedure.

Patients and methods: Seventeen unselected patients (16 males and one female), age (median (range)) 19 (10-29) years, were examined 18 (9 25) years after Mustard's procedure for TGA (age at operation $14(10-48)$ months). RVM was measured by magnetic resonance imaging using breath-hold cinematographic gradient-echo technique with the imaging plane in the true short axis of the right ventricle (slice thickness $6 \mathrm{~mm}$, no inter-slice gaps, TR 55 msec). VO2max was determined by an individualized incremental bicycle ergometer test with continuous measurement of metabolites. Criteria for maximal exercise were exhaustion, $\mathrm{VO} 2$ plateau reached or $\mathrm{VCO} 2 / \mathrm{VO} 2>1.1$.

Results: RVM index was (mean (SD)(range)) $94.5 \mathrm{~g} / \mathrm{m} 2$ (16.9) (56.3-120) and $\mathrm{VO} 2 \max$ was $1.90 \mathrm{l} / \mathrm{min}(0.73)$ $(=31.6 \mathrm{ml} / \mathrm{min} / \mathrm{kg}(6.95))$. Furthermore, we found a positive correlation between RVM index and VO2max $(r=0.57, p=0.02)$. Discussion: In contrast to left-sided ventricular systolic dysfunction, where the degree of myocardial hypertrophy correlates with the severity of heart failure, this study indicates that increased RVM index after atrial switch procedures for TGA results in better ventricular function as measured by exercise performance. However, it is uncertain what causes the large range in RVM index in Mustard operated patients. One explanation could be the angiotensin-converting-enzyme genotype, which is known to be related to the degree of myocardial hypertrophy in other settings of increased ventricular workload.

\section{P200}

\section{Lung function and aerobic capacity in adolescents and} adults with various congenital heart diseases

F.M. Pichio, S. Specchia, G.V. Coutsoumbas, M. Bonvicini,

A.M. Boiani, G. Bronzetti, R. Formigari

Pediatric Cardiology Bologna, Bologna, I

Objective: At present particular interest is addressed to evaluate cardiopulmonary performance in patients (pts) with congenital heart diseases. The ain of our study was to assess static lung function and cardiopulnunary performance in a selected series of pts examined in our laboratory.

Patients and methods: 111 untrained pts, 50 male, 61 female, age $25.6 \pm 16.8$ yrs (range $6-68 \mathrm{yrs}$ ), all in I-II NYHA functional class, undergone maximal cardiopulmonary exercise test, were subdivided into 5 major diagnostic groups, including 29 pts with corrected atrial septal defect (ASD), 10 unoperated Ebstein's anomaly, 43 operated Tetralogy of Fallot (TdF), 18 transposition of great arteries repaired with atrial switch (Mustard/Senning), and 11 Fontan (7 "classic" and 4 "modified" procedures). The data expressed as (\%) were compared with predicted normal values.

Results: The data obtained in the different groups of pts (mean \pm SD), compared by one way ANOVA analysis are reported in the Table.

Conclusions: 1. Lung function was normal in our Ebstein and corrected ASD pts, diminished in other diagnostic groups, particularly in Fontans (both at rest and in dynamic conditions) but the exercise capacity was reduced in all groups (less in ASD, maximally in Fontan); 2. Fontan pts also showed evident chronotropic incompetence; 3. "Asymptomatic" unoperated Ebstein's pts showed better, but not statistically significant, performance than operated Mustard/Senning and Fontan ( \pm modified) pts; 4 . No functional differences we observed between "classic" and "modified" Fontan pts; 5 . Diminished values of lung function and peakHR contribute to the reduction of exercise capacity specially in pts with Fontan;

The significance and the clinical implications of our results will be discussed with reference to other recent reports.

\section{P201}

The current status of adult congenital heart disease: a retrospective study of an International Society of Adult Congenital Cardiac Disease database M. Sijbom, M.E. King, M. de Moor, A. Marelli

Massachusetts General Hospital, Boston, USA

Aim: The International Society of Adult Congenital Cardiac Disease created a database to assess the current status of adults with congential heart disease. A total of 1280 patients were registered from 10 centers ( 9 in the United States and 1 in Japan).

Methods: Information from a standardized questionnaire was obtained on patients seen in participating centers in 1996 and entered into an Ingress database. The data were abstracted into an Excel database for further analysis. The questionnaire addressed demographics, cardiac anatomy, operative status, complications, NYHA functional class, medications and social history.

Results: The median age was $30+1-9.4$ years (range $17-93 \mathrm{yrs}$ ). There were 623 males and 657 females. The most common lesion was a septal defect (VSD $\mathrm{n}=346$, ASD $\mathrm{n}=268$ ), followed by Tetralogy of Fallot $(n=149)$, aortic coarctation $(n=96)$ and patent ductus arteriosus $(n=79)$. Many of the patients had corrective $(n=685)$ and/or pallitative $(n=226)$ surgery; 404 patients were unoperated. The majority of the complications included heart failure $(n=106,8.3 \%)$, endocarditis $(n=57,4.5 \%)$, and arrhythmia $(n=293,22.9 \%)$ which included 37 patients with complete heart block. Many patients were employed $(n=594)$. married $(n=451)$ and had children $(n=314)$. Of the 657 females, 181 had a total of 382 pregnancies of which 68 were aborted, and 261 were delivered at term.

Conclusions: This data would suggest that adults now followed in adult congenital cardiac programs have had the benefit of surgical interventions, have good functional status and lead constructive lives. Although there is a significant incidence of complications. 
current progress in early diagnosis and treatment of children with congenital heart defects may further improve the longterm outlook for adults with these disorders.

\section{P202}

Neurohumoral activation at rest and during exercise in adult patients with repaired tetralogy of Fallot

G.R. Veldman, C. Silversides, G.D. Webb, M. Mullen, S. Hechter,

I. Howe, N. Merchant, S. Siw, P. Liu, B.W. Mc Crindle

Hospital for Sick Children Toronto, TCCCA, Rochester, LSA

Background. Neurohumoral activation in the setting of pulmonary RV dysfunction is poorly understood. We sought to characterize resting and maximal voluntary exercise proANP (atrial natriuretic peptide), proBNP (brain natriuretic peptide) and ET I (endothelin) levels in adult patients with repaired Tetralogy of Fallot.

Patients \& methods. 20 consecutive patients $>18$ years, were prospectively evaluated with clinical assessment, 12-lead ECG, 2D-echo, MRI and graded bicycle cardiopulmonary exercise testing. Tests were done in a standardized fashion.

Results. Median age was 30) years (range 20-48), and $10(53 \%)$ were female. Median age at repair was 7.5 years (range 3-21).

Exercise induced proANP elevation correlated well with longer cardiopulmonary bypass time $(r=0.46, p=0.004)$, harder work of breathing $(r=0.35, p=0.04)$, higher VO2 peak $(r=0.40$, $p=0.016)$ and greater exercise workload $(r=0.42, p=0.013)$. Exercise related increases in proBNP correlated well with higher VO2 peak $(r=0.31, p=0.1) 35)$, and ECG markers of right heart hypertrophy i.e. QRS axis $(r=0.40, p=0.029)$ and greater $\mathrm{R}$-wave height in V1 $(\mathrm{r}=0.35, \mathrm{p}=0.04)$. ET I levels at rest rended to correlate with greater exercise workload $(r=0.34$. $p=0.06)$, and reduced work of breathing $(r=0.31, p=0.09)$.

Conclusions. There is significant neurohumoral activation in some adult patients with repaired tetralogy of Fallot both at rest and during exercise. Perioperative factors, current hemodynamic burden, and exercise stress may influence neurohormonal profiles in these patients.

\begin{tabular}{|c|c|c|c|c|c|}
\hline & $\begin{array}{l}\text { Resting } \\
\text { Mean } \pm \text { SD } \\
(\text { trmol/l) }\end{array}$ & $\begin{array}{l}\text { Exercise } \\
\text { Mean } \pm \mathrm{SD} \\
(\text { fnol } / \mathrm{l})\end{array}$ & $P$ & $\begin{array}{l}\text { Normal } \\
\text { Range } \\
(\text { fmol/l) }\end{array}$ & $\begin{array}{l}\text { Rest vs. } \\
\text { Exercise } \\
\text { Abnormal } \\
\text { counts } \%\end{array}$ \\
\hline วroANP & $2867 \pm 1930$ & $3510 \pm 1805$ & $0.001^{\star \star}$ & $103-1.359$ & 68 vs. 79 \\
\hline rroBNP & $350 \pm 125$ & $4+7 \pm 254$ & $0.075^{\star \star}$ & $126-366$ & 47 vs. 68 \\
\hline Endothelin I & $1.5 \pm 1.3$ & $1.5 \pm 1.4$ & 0.58 & $0.12-0.7$ & 47 vs. 47 \\
\hline
\end{tabular}

\section{P203}

Partial and total anomalous pulmonary venous connection in the fetus: two-dimensional and spectral doppler echocardiographic findings

E.R. Valsangiacomo, L.K. Hornberger, C. Barrea, J.S. Smallhorn,

S.J. Yoo

Lniversity Children's Hospital, Zurich, $\mathrm{CH}$

Background: Prenatal diagnosis of total (TAPVC) or partial PAPVC) anomalous pulmonary venous connection in isolation or associated with other cardiac disease is important for appropritte prenatal counseling and perinatal management.

Jbjectives: We sought to assess the echocardiographic clues to the etal diagnosis of TAPVC and PAPVC in a cohort of affected fetuses. Methods: We retrospectively reviewed 29 fetal echocardiograms perormed in 16 pregnancies with fetal TAPVC or PAPVC, systematisally analyzing heart chamber size, presence of a confluence behind he left atrium or of a vertical vein, and Doppler flow patterns.
Results: Prenatal diagnosis was made at a mean gestational age of 297 weeks. TAPVC of supracardiac or infracardiac types was found in 5 cases each and of mixed type in one. PAPVC was diagnosed in 5 fetuses, 4 of whom had scimitar syndrome. Ten fetuses had an additional major cardiac defect, including hypoplastic left heart syndrome and right atrial isomerism. In 3 cases the prenatal diagnosis was only made at follow-up assessment. Among TAPVC cases, visualization of a confluence behind the left atrium (10/11) and a vertical vein $(11 / 11)$ were the most consistent echocardiographic clues. Dextrocardia and a small right pulmonary artery suggested scimitar syndrome. The diagnosis was confirmed postnatally or at autopsy in 12 cases. In 6 fetuses with TAPVC and obstruction confirmed postnatally, continuous turbulent flow in the vertical vein and monophasic continuous flow in the pulmonary veins were demonstrated by color and spectral Doppler. Conclusions: Fetal echocardiography permits prenatal diagnosis of TAPVC or PAPVC. Spectral and color Doppler provide clues to the presence of obstructed pulmonary venous pathway.

\section{P204}

Assessment of myocardial perfusion by magnetic resonance first-pass perfusion imaging after the arterial switch operation: a preliminary assay M.P. Magalhaes, M.A. Sampaio Nunes, E. da Cruz, N. Jalles Tavares, A. Cidraes, M. Ferreira, E. Silva, L. Rosario, J. Fernandes Hospital da Cruz Vermelha Portuguesa, Lisbon, $P$

Purpose: Coronary disease after Jatene's switch operation is traditionally assessed by effort tests, ecocardiogram, angiography, nuclear imaging or Positron Emission Tomography. Magnetic Resonance First-pass Perfusion Imaging (MRFP) appears to have an encouraging potential in adults. Aiming to develop a noninvasive tool for follow-up of coronary hypoperfusion, we studied myocardial blood flow by MRFP and angiography in children.

Materials and methods: Eighteen patients, 11 male (61.1\%) and 7 female $(39.9 \%$ ), aged between 40 and 99 months (mean $=75.4$ months; median $=76$ months), with weights between 14 and $32 \mathrm{~kg}($ mean $=21.6 \mathrm{~kg} ;$ median $=21 \mathrm{~kg}$ ) were prospectively studied with ecocardiogram, ECG, cardiac catheterisation and MRFP. Cardiac defects included: Transposition of the Great Arteries (TGA) (9, 50\%), TGA with ventricular septal defect (VSD) (5, $27.8 \%)$, Taussig-Bing complex (2,11.1\%), TGA with VSD and aortic coarctation $(1,5.5 \%)$, and TGA with VSD and subpulmonary obstruction $(1,5.5 \%)$. Two patients (11.1\%) had had a later left coronary internal thoracic mammary grafting. Magnetic Resonance images were performed with a $1.5 \mathrm{~T}$ magnet (CV GE Medical Systems) using axial and sagital planes. After assessment of resting cardiac function, myocardial hyperaemia was induced by IV Dipiridamol, followed by the injection of Gadoliniumdiethylenetriamine pentaacetic acid bismethylamide (Omniscan, Nycomed Imaging AS). Myocardial first-pass kinetics and detection of signal enhancement in areas of irreversible damage were monitored. MR images were reviewed by two authors unaware of any other data.

Results: Ventricular colume, mass and ejection were normal in all patients. Eight patients $(44.5 \%)$ had a subendocardial hypoperfusion area in the mediobasal segment: in all but one, this hypoperfusion did not correlate with any segmentary abnormalities or with coronary disease, and in 6 of them, this fact might be explained by the presence of a VSD patch.

Conclusion: In this small series we cannot draw definitive conclusions; however, we can state that MRFP is a feasible, safe, nontoxic, non-invasive and very attactive technique in children, that seems to be highly sensitive but poorly specific for early detection 
of myocardial hypoperfusion in patients after the switch operation. We are undergoing a learning curve experience by studying more patients in order to elucidate the potentials of this technique.

\section{P205}

Myocardial blood flow and coronary flow reserve in children with "normal" subepicardial coronary arteries after Kawasaki disease assessed by positron emission tomography

M. Hauser, A. Khn, F.M. Bengel*, K. Lohner, S. G. Nekolla* M. Schwaiger ${ }^{*}$, J. Hess

German Heart Centre Munich, Dept. of Paediatric Cardiology, Munich, D

Objectives: Myocardial blood flow (MBF) was investigated quantitatively in children (13.3 SD 4.02 years) with "resolved" coronary involvement and angiographically normal epicardial coronary arteries. Background: Kawasaki syndrome is an acute inflammatory disease, affecting the arterial walls in form of a panvasculitis; the long-term integrity of the coronary arteries remains a matter of concern. Methods: 7 asymptomatic children with Kawasaki syndrome had ECG (rest/exercise), echocardiography and positron emission tomography 9.2 SD 5.01 years after the acute illness. Myocardial perfusion was assessed by NH3-PET at rest and after vasodilatation with adenosine; the results were compared with 10 healthy volunteers (26.1 SD 6.3 years). Results: No patient had signs of myocardial ischaemia; ECG rest/exercise was normal without signs of coronary insufficiency; on echocardiography ventricular function was normal without dyskinetic areas or signs of enlargement or stenosis of the proximal coronary arteries. There was no statistical significant difference between patients and healthy volunteers in $\mathrm{MBF}$ at rest $(0.84 \mathrm{SD} 0.22$ v.s. $0.77 \mathrm{SD} 0.17 \mathrm{ml} / \mathrm{g} / \mathrm{min})$, whereas MBF after vasodilatation (2.56 SD 0.90 vs. $3.10 \mathrm{SD} 0.8 \mathrm{ml} / \mathrm{g} / \mathrm{min}$ ) and coronary flow reserve (CFR) (2.99 SD 0.36 vs. 4.09 SD 1.01) was significantly attenuated in the Kawasaki group. No stress induced perfusion defects could be detected within the myocardium. Conclusion: In children with a history of Kawasaki disease and angiographically normal subepicardial coronary arteries there is a significant attenuation of $\mathrm{MBF}$ after vasodilatation and a significant reduction of CFR. Impairment of the vasoreactive ability may indicate a residual damage of the coronary arteries and may be a risk factor for atherosclerosis in adulthood.

\section{P206}

Microcirculation and myocardial blood flow in patients late after atrial repair of transposition of the great arteries

M. Hauser, A. Khn, A. Lammers, F.M. Bengel, S. G. Nekolla, H. Kaemmerer, M. Schwaiger, J. Hess

German Heart Centre Munich, Dept. of Paediatric Cardiology, Munich, D

Background: Operative palliation of children with transposition of the great arteries (TGA) using a rerouting of systemic and pulmonary venous return was widely practiced. The occurrence of systemic ventricular dysfunction after atrial repair of TGA has been well described and symptomatic right ventricular dysfunction is evident in at least $10 \%$ of patients at ten years. Little attention has been given to myocardial perfusion and the possible role of myocardial ischemia in the pathogenesis of right ventricular dysfunction.

Methods: We investigated 22 adolescents with TGA (age: $22.6 \mathrm{SD}$ 4.7 years), 21.2 SD 3.8 years after atrial repair (Mustard/Senning). All patients had cardiac catheterization; myocardial perfusion was assessed by NH3-positron emission tomography (PET) at rest and after vasodilatation with adenosine; the results were compared with 10 healthy adults (26.1 SD 6.3 years)
Results: Angiography excludes any stenosis of the subepicardial coronary arteries. Compared to normals myocardial blood flow (MBF) after maximal vasodilatation (TGA: 1.70 SD 0.59 vs. normals: $3.10 \mathrm{SD} 0.85 \mathrm{ml} / \mathrm{g} / \mathrm{min}$.) and coronary flow reserve (CFR) (TGA: 2.44 SD 0.60 vs. normals: 4.1 SD $1.01 \mathrm{p}<0.01$ ) were significantly attenuated in the TGA group; no persistent or reversible perfusion defects could be detected in any of the patients.

Conclusion: Attenuated myocardial blood flow parameters and impairment of microcirculatiuon are common findings in patients with TGA after atrial repair; this may be important in the pathogenesis of late ventricular dysfunction and deterioration of the systemic ventricle.

\section{P207}

Non-invasive quantification of stent stenosis using MRI

T. Kuehne, S. Weiss, M. Gutberlet, C. Lilje, J. Weil

University Hospital Hambug, Pediatric Cardiology, Hamburg, D

Purpose: Development of stent stenosis in the lumen of endovascular stents occurs frequently. The noninvasive application of MRI for the assessment of stent patency would be desirable but is limited, because artefacts of clinically available stents can be misinterpreted as stent obstruction or occlusion. The purpose of this study was to (1) develop a design concept for a stent which is characterized by minimal artefacts and to (2) test the feasibility to quantify non-invasively stent stenosis using MRI.

Material and methods: The stents evaluated had a diameter of 8 and $18 \mathrm{~mm}$. The wire mash of the stent alloy was composed of a weakly paramagnetic metal and designed in a fashion that minimizes shielding of MR radio frequency pulses.

The stents were integrated in a well-controlled flow phantom. The lumen of the stents were partially occluded with a water gel to create an artificial vascular stenosis of 50 and $75 \%$. The degree of stent stenosis was assessed on T1 weighted 3D-Gradient echo (T1-GRE) images. Pressure gradients over the stenosis were measured using velocity encoded cine (VEC) MRI. All imaging was performed using an 1.5 Tesla imager (Intera, Philips, Best).

Results: On T1-GRE images a negligible (10.4 pixel wide) ring of artefact was noted at the borders of the stents. The obstructed section of the stent could be unequivocally differentiated from the non-obstructed section on T1-GRE. The agreement between MRI and the known degree of stenosis was excellent $(r=96$, $\mathrm{p}<0.01)$. Pressure gradients as measured with VEC MRI were 481.9 and $791.4 \mathrm{mmHg}$ compared to pressure gradients of 50 and $80 \mathrm{mmHg}$ as calculated from the known output of the pump and the surface area of the used tubing of the flow phantom.

Conclusion: The results of this study imply that the evaluated stents seem to be well suited to determine accurately, reliably and non-invasively in-stent stenosis using MRI. The findings of this in-vitro study will have to be validated in animal studies, which are currently underway.

\section{P208}

Balloon angioplasty for aortic recoarctation: age related aspects

G. Agnoletti, A. Borghi ${ }^{\star}$, T. A Massih, P. Bonhoeffer, D. Bonnet, D. Sidi Necker Enfants Malades, Paris, $F$

Balloon angioplasty (BA) is widely accepted as the elective technique to relieve aortic recoarctation, as it has low mortality, low morbidity and excellent results at follow up. Some concern exists on its possible increased risk in small children. We retrospectively examined 58 children undergoing consecutive BA of aortic recoarctation: 30 had 1-12 months (group A) and $28>1$ year (group B). The presence of VSD or other cardiac anomalies was 
highly associated with younger age $(p<0.001)$. The morphology of recoarctation was similar in the two groups. Systolic hypertension was present in $70 \%$ of group A and 32\% of group B ( $p<0.001)$. Systolic blood pressure was highly correlated with initial gradient $(r=0.81, p<0.01)$, whilst age was inversely related with initial gradient $(r=-28, p<0.05)$. The procedure was successful (final gradient $<20 \mathrm{~mm} \mathrm{Hg}$ ) in $87 \%$ of group A and $86 \%$ of group B. Age was not related with residual gradient nor with the percentage increase in diameter of the coarcted segment. After BA systolic hypertension was still present in $37 \%$ of group $A$ and $25 \%$ of group $B(p<0.05)$. Three complications occurred in group A and 7 in group B ( $p<0.05)$. Conclusions: Our results show that 1$)$ earlier recoarctation occurs in complex forms, 2) young children are more likely to be hypertensive, either before or after $\mathrm{BA}, 3$ ) $\mathrm{BA}$ has the same success rate in children aging $<$ or $>1$ year, 4) BA has not an higher risk in children $<1$ year.

\section{P209}

Brain perfusion assesed by $99 \mathrm{mTc}$-ECD imaging in patients with vasivagal syncope during tilt test R. Olgunturk, L. Turan, FS. Tunaoglu, N. Gokcora, N. Ilgin, S. Kula Gazi University, Medical Faculty, Pediatric Cardiology Dpt., Ankara, TR

Background: Brain perfusion may be impaired in patients with vasovagal syncope (VS). A previous study has reported predominantly frontal cortical perfusion abnormality in brain perfusion following the tilt provocation test in VS patients.

Objective: To assess the brain perfusion in patients with VS using by $99 \mathrm{mTC}-\mathrm{ECD}$ SPECT imaging of the brain and search for regional selectivity in perfusion pattern following tilt testing in patients with VS. Design: $99 \mathrm{mTC}$-ECD SPECT was carried out in 18 patient with VS after injection of the tracer immediately following syncope in 13 and pre-syncope in 5 during a $45 \mathrm{~min} 60$ degree head up tilt test. Data was acquired $1 \mathrm{hr}$ after the injection and 64 frames were obtained in $128 \times 128$ matrix with high resolution parallel hole collimation. Data storage and reconstruction of transverse images were performed by use of a computer system (Optima NX GE) coupled with the SPECT camera using a Butterworth smoothing filter. Attenuation corrections were performed using $M=0.12$. A total number of 12 sector regions of interest were placed on the two hemispheres and region/cerebellum ratios were calculated from three consecutive ( 2 pixel thick) slices (upper, middle, lower) for semiquantative estimation of the cerebral blood flow.

Results: While there were no statistically significant reductions were observed in a single dominant region compared to the other regions examined using ANOVA, area of lowest perfusion reserve were found most frequently in the prefrontal-frontoparietalanterior parieto-orbito frontal and anterior temporal regions. Region cerebellum ratios were ranging from 0.85 to 1.15 in different cortical areas.

Conclusion: Patients with tilt positive have relatively normal global brain perfusion index with reduction of flow predominantly involving the prefrontal-frontoparietal-anterior parietalorbito frontal and anterior temporal regions in this group of tilt positive patients.

\section{P210}

Rapid noninvasive shunt quantification by magnetic resonance imaging in children

P. Beerbaum, H. Krperich, P. Barth, J. Gieseke, H. Esdorn, H. Meyer

Clinic for Congenital Heart Disease, Heartcenter NRW,

Bad Oeynhausen, D

Background: Noninvasive left-to-right shunt quantification in children by phase-contrast cine magnetic resonance imaging
(PC-MRI) is accurate, but time-consuming and spatial resolution is limited. We sought to evaluate rapid and high-resolution PC-MRI techniques using sensitivity encoding (SENSE).

Methods and results: In 20 children with an atrial septal defect, blood flow rate in the pulmonary artery and aorta was determined by cine phase-contrast MRI combined with parallel k-space readout by use of sensitivity enconding (PC-MRI/SENSE). VectorECG was used in stead of standard ECG to improve phase reordering during retrospective gating. The stroke volumes and the ratio of pulmonary to aortic flow $(\mathrm{Qp} / \mathrm{Qs})$ were compared with results from a standard retrospectively-gated cine phasecontrast PC-MRI pulse sequence that was recently evaluated in children with left-to-right shunt. Mean acquisition time for PCMRI/SENSE was 15-30 sec., depending on heart rate, spatial resolu-tion and SENSE factor, and 2.5-3.5 minutes by standard cine PC-MRI, respectively. Pulmonary and aortic stroke volumes and the Qp/Qs ratio obtained by PC-MRI/SENSE as well as highresolution PC-MRI with and without SENSE agreed well with the standard cine PC-MRI protocol. The interobserver variability was low for all pulse sequences. Accuracy of research and standard PC-MRI protcols were confirmed by in vitro experiments using a calibrated pulsatile flow phantom constructed by use of a ventricular assist device (MEDOS[R]).

Conclusions: In pediatric patients with left-to-right shunt, rapid and accurate shunt quantification is possible by PC-MRI combined with SENSE, compared with standard PC-MRI measurements. High-resolution PC-MRI with SENSE may be adaequate for smaller children and infants, pending confirmation in a larger population.

P211

Wall stress misrepresents afterload in ventricles with abnormal chamber geometry

T.L. Gentles, S.D. Colan

Green Lane Hospital, Auckland, NZ

Background: Wall stress (WS), although commonly used as an index of LV afterload, fails to take into account forces generated within the wall that oppose fiber thickening during contraction.

As a result, WS may misrepresent average fiber stress (FS) - the actual shortening force exerted by individual fibers - particularly in the presence of an abnormal thickness:dimension ratio.

Methods: Echocardiograms were obtained in 4 groups of children and young adults (age 12.76 .0 years) with a wide range of values for LV mass and/or end-diastolic thickness:dimension ratio (h/D). Diagnoses were: aortic stenosis (AS), $\mathrm{s} / \mathrm{p}$ coarctation (COA), $\mathrm{s} / \mathrm{p}$ adriamycin treatment (ADR), and severe aortic and/or mitral regurgitation (AR/MR). End-systolic WS and FS, LV mass, and $\mathrm{h} / \mathrm{D}$ were calculated and expressed as $\mathrm{z}$-scores relative to a normal population $(\mathrm{n}=305)$.

Results. There were significant differences between WS and FS in all groups excepting ADR (Table - mean z-scores values. ${ }^{\star} \mathrm{p}<0.05$ compared to normal population. \#p $<0.05$ WS compared to FS.)

The difference between WS and FS z-scores was extreme when $\mathrm{h} / \mathrm{D}$ was elevated (WS $<<\mathrm{FS}$ ) or reduced (WS $>>\mathrm{FS}$ ) (WS $\left.\mathrm{FS}=0.6^{\star}(\mathrm{h} / \mathrm{D})-1.04-4.16, \mathrm{r}=0.82, \mathrm{p}<0.001\right)$

Conclusions: Fiber stress, the force opposing fiber shortening, is overestimated by WS in thin walled ventricles and underestimated by WS in thick walled ventricles. Because of this, results of stressshortening analysis based on WS will be invalid in patients with abnormal ventricular geometry. 
P212

Echo-doppler assessment of the biophysical properties of the aorta in young patients with Marfan Syndrome T.J. Bradley, J.E. Potts, A. DeSotiza, M.T. Potts, T. Hishitani, G.G.S. Sandor

Division of Cardiology, B.C.'s Children's Hospital, Vancowver, BC, CDN

Introduction: Abnormal aortic root stiffness has been demonstrated in Marfan Syndrome, even without dilation of the ascending aorta. Vascular impedance best describes the load imposed on the heart in a pulsatile flow system. We have previously described an Echo-Doppler method to measure pulse wave velocity (PWV), aortic input $(\mathrm{Zi})$ and characteristic $(\mathrm{Zc})$ impedances, the arterial pressure-strain elastic modulus (Ep) and the arterial wall stiffness index (index). We report the results of a study assessing the biophysical properties of the aorta in young Marfan patients using this method. Methods: 16 Marfan patients (median age 15.4 [range 9.5-18.6] years) and 19 healthy controls (age 13.2 [7.4-19.4] years) were studied. After measuring the aortic annulus, pulse Doppler was recorded with the sample volume just above the aortic valve $(\mathrm{A} \circ \mathrm{V})$ and distally in the descending aorta. The length (L) between these two points was measured. The time to onset of the two Doppler tracings from the ECG q-wave was measured and the difference used to determine the transit time (TT) of the pulse wave. Ascending aorta diameters in systole (Ds) and diastole (Dd) were measured using $\mathrm{M}$-mode. Aortic peak flow = CSA $\times$ AoV; $\quad$ PWV $=\mathrm{L} / \mathrm{TT} ; \quad \mathrm{Zi}=(\mathrm{BPs}-\mathrm{BPd}) / \mathrm{CSA} \times \mathrm{AoV} ;$ $\mathrm{Zc}=(\mathrm{PWV} \times \mathrm{p}) / \mathrm{CSA} ; \mathrm{Ep}_{\mathrm{p}}=[(\mathrm{BP} \mathrm{s}-\mathrm{BPd}) /(\mathrm{Ds}-\mathrm{Dd})] \times \mathrm{Dd} ;$ and index $=\ln \{[(\mathrm{BPs} / \mathrm{BPd}) /(\mathrm{Ds}-\mathrm{Dd})] \times \mathrm{Dd}\}$ were calculated. The data were analysed using univariate analysis and non-parametric analysis of variance. Results: There were no differences in the median age, weight or BSA, but Marfan patients were taller (174 vs $161 \mathrm{~cm} ; \mathrm{p}<0.009)$. Systolic and diastolic blood pressures were lower in Marfan patients (101 vs $114 \mathrm{mmHg}$; $p<0.007$ and 65 vs $78 \mathrm{mmHg}$; $<0.001$, respectively), but pulse pressures were similar. Aortic diameters, Ds and Dd, were similar (2.6 vs 2.6 and 2.3 vs 2.2 , respectively). PWV ( 495 vs $365 \mathrm{~cm} / \mathrm{s} ; \mathrm{p}<0.02)$, Ep (310 vs 216 dynes $^{\star_{\mathrm{s}} / \mathrm{cm} 5}$ ) and the index $(2.61$ vs $2.11 ; \mathrm{p}<0.003)$ were greater in Marfan patients. $\mathrm{Zi}$ and $\mathrm{Zc}(119 \mathrm{vs} 131$ and $148 \mathrm{vs}$ 143 dynes $^{\star} \mathrm{s} / \mathrm{cm} 5$, respectively) were similar. Conclusions: This echo-Doppler study demonstrated abnormal PWV and aortic stiffness indices and normal vascular impedances in young Marfan patients. Even though the ascending aorta was not dilated in this cohort of Marfan patients, the biophysical properties of their aorta are abnormal.

\section{P213}

Time-resolved 3D-magnetic resonance angiography in the assessment of pulmonary vascular anomalies J. Eichhom, C. Fink ${ }^{\star}$, M. Bock*, S. Delorme*, K. Brockmeier, H.E. Ulmer

Department of Pediatric Cardiology, University Heidelberg, Heidelberg, $D$

Introduction: The standard procedure for the reliable visualisation of pulmonary vascular anomalies in pediatric patients is conventional catheter angiography. Contrast-enhanced 3D-MRA with scan times of usually over 20 seconds is often of limited diagnostic value due to breathing artefacts and the venous overlay. The results of a time-resolved 3D-magnetic resonance angiography in 5 pediatric patients $(8-15 \mathrm{y})$ with suspected anomalies of the pulmonary vessels are demonstrated: pulmonary artery sling (1), pulmonary arteriovenous malformation (3), ectasy of pulmonary trunc (1).

Method: For the evaluation of the pulmonary vasculature a timeresolved contrast-enhanced magnetic resonance angiography (MRA) was performed on a $1.5 \mathrm{~T}$ whole-body scanner (Magnetom
Vision, Siemens). The imaging protocol included an ultrafast 3D FLASH sequence with asymmetric $k$-space sampling and a partially self-refocussing radiofrequency pulse (TR/TE $=2.3 / 0.9 \mathrm{msec}$, flip angle $=40$, scan time $=6.22 \mathrm{sec}, 0.2 \mathrm{mmol} / \mathrm{kg}$ body weight $\mathrm{Gd}-$ DTPA i.v.). In total 4 to 5 data sets were acquired in a single breathhold. Three-dimensional reconstructions (volume-rendering) of contrast-enhanced MRA dynamic acquisitions demonstrated different phases of the contrast bolus flowing (pulmonary artery phase, arteriovenous phase, aortal phase).

Results: Time-resolved 3D-magnetic resonance angiography showed high image quality and was of diagnostic value in all patients. In one patient $(8 \mathrm{y})$ an aberrant left pulmonary artery passing posteriorly to the trachea to the left (pulmonary artery sling) was diagnosed. In three patients pulmonary arteriovenous malformations and in one of them an aortopulmonary collateral artery were demonstrated. In the fifth patient (16y) MRA confirmed the diagnosis of an ectasy of pulmonary artery trunc (diameter: $4,5 \times 3,6 \mathrm{~cm}$ ).

Conclusion: Time-resolved contrast-enhanced 3D-magnetic resonance angiography in future may becames the modality of choice as a minimal invasive and nonionizing technique for the diagnosis pulmonary vascular anomalies. These findings contributed essentially to the decision for an interventional or surgical approach. In this limited number of patients the diagnostic value was comparable to conventional catheter angiography.

\section{P214}

Closure of patent ductus arteriosus in patients with pulmonary hypertension and bodyweight $<8 \mathrm{~kg}$ using the Amplatzer occluder

W. A. K. Radtke, M. Dilaurar, V. Bandisode

Medical University' of South Carolina, Charleston, LSA

In order to determine safety and efficacy of transcatheter occlusion of patent ductus arteriosus (PDA) using the Amplatzer Duct Occluder in small patients, we report the single center experience in patients weighing $<8 \mathrm{~kg}$. Device occlusion of the PDA was attempted in 10 patients, aged 3-15 months (median 7 months), weighing $3.8-7.5 \mathrm{~kg}$ (median $6.0 \mathrm{~kg}$ ) and accomplished in all. $\mathrm{QP} / \mathrm{QS}$ was 2.0-5.7 (mean 3.4) and the smallest ductus diameter was $3.0-5.4 \mathrm{~mm}$ (mean $4.5 \mathrm{~mm}$ ). Left ventricular end diastolic pressure was elevated in most patients, ranging from 8 to $25 \mathrm{mmHg}$ (mean $15.3 \mathrm{mmHg}$ ). Pulmonary artery pressure ranged from $40-100 \%$ systemic (mean 59.6\%). We used $4 / 5 \mathrm{~mm}, 4 / 6 \mathrm{~mm}$ and $6 / 8 \mathrm{~mm}$ occluders. Median procedure time was $120 \mathrm{~min}$ (42-153 min) with a median fluoroscopy time of $19.2 \mathrm{~min}$ (13.5-28.4 min). There were no device embolizations or other major complications. One parient had protrusion of the device into left pulmonary artery and two patients had protrusion into the distal aortic arch with pressure gradients of $10 \mathrm{mmHg}$ or less. Complete occlusion was achieved in $5 / 10$ pacients $(50 \%)$ after $10 \mathrm{~min}$, in $9 / 10$ $(90 \%)$ after $12-24$ hours, in $8 / 8$ patients $(100 \%)$ after 6 months and in $3 / 3$ patients $(100 \%)$ after one year. There were no late complications. We conclude that transcatheter occlusion of PDA is safe and effective in patients under $8 \mathrm{~kg}$ using the Amplatzer Duct Occluder.

\section{P215}

Functional assessment of postsurgical coarctation by magnetic resonance imaging

C. Rose, A. Wessel, E. Castillo, W. Rusicheuski

Klinik und Poliklinik fr Kinder- und Jugendmedizin, Dresden, D

Purpose: To compare three methods of non-invasive functional assessment after coarctation repair and to evaluate their reliability. $\mathrm{CW}$-Doppler, oscillometric blood pressure gradient and flow 
quantification by velocity encoded MRI were contrasted to the results of morphologic measurements.

Methods: 54 patients (aged 6 to 36 years) were assessed by MRI, CW-Doppler and oscillometric pressure gradient 3 to 31 years after coarctation repair. CW-Doppler measurements were taken from the suprasternal position. MRI was performed to assess the morphologic result of the aortic arch and to quantify flow distal to the narrowest segment. This volume flow divided by the cross-sectional area of the narrowest segment equals the mean cross-sectional velocity. The diameter of the narrowest segment were compared to growthdependent normal values for calculation of the degree of stenosis. Results: Linear correlation of CW-Doppler and oscillometric pressure gradient with the morphologic degree of stenosis were weak. $(r=0,4)$. Mean cross-sectional velocity correlates much better by using a function with negative exponent $(r=0,78)$. The differentiation between low-grade and moderate stenoses was significantly better by mean cross-sectional velocity( $p<0,0001)$ than $C W-$ Doppler $(p<0,01)$ or blood pressure gradient (not significant). Conclusion: Mean cross-sectional velocity measured by MRI is superior to $\mathrm{CW}-\mathrm{I}$ oppler and oscillometric pressure gradient in moderate coarctation.

\section{P216}

Three-dimensional echocardiographic evaluation of atrioventicular valves enhances surgical decision making in pediatric patients.

C. Barrea ${ }^{\star}$ J.G. Coles, W.G. Willians, G.S. Van Arsdell, J.F. Smallhorn Cliniques Lniversitaires Saint LuC - UCL, Brussels, B. \& Toronto, CND

Background: The function and competency of atrioventricular valves (AVV) are important prognostic factors in patients with congenital heart disease. Assessment of the AVV by the surgeon is difficult on an empty and non-beating heart. Two-dimensional echocardiography is a good diagnostic tool, it however has limitations and requires three-dimensional mental reconstruction. Three-dimensional echocardiography (3DE) permits the creation of surgical views and provides physiologic information.

Methods: Over a 13-month period, 11 patients (median weight $20.3 \mathrm{Kg}$, range $4-66 \mathrm{Kg}$ ) underwent a $3 \mathrm{DE}$ evaluation of their AVV either by TEE $(n=9)$ or by TTE $(n=2)$ prior to and after surgery with peroperative confirmation using a head-mounted camera. This study evaluates the accuracy of dynamic assessment of AVV and compares the $3 \mathrm{D}$ reconstructed images with the surgical findings. We looked at the mobility and the integrity of each individual leaflet and assessed the sites and mechanisms of AVV regurgitation.

Results: The left AVV was assessed in 8 cases (7 AVSD, 1 vegetation) and the tricuspid valve in 3 cases ( 1 Ebtsein's, 1 vegetation, 1 iatrogenic tear). 3D reconstruction was possible for all patients for at least 1 dataset. Mainly enface views from the AVV were obtained. Better quality information was obtained by TEE. In all cases, information obtained by $3 \mathrm{DE}$ was superior to that obtained by $2 \mathrm{DE}$. We found a good correlation between the 3D reconstructed images with the surgical findings, however, one

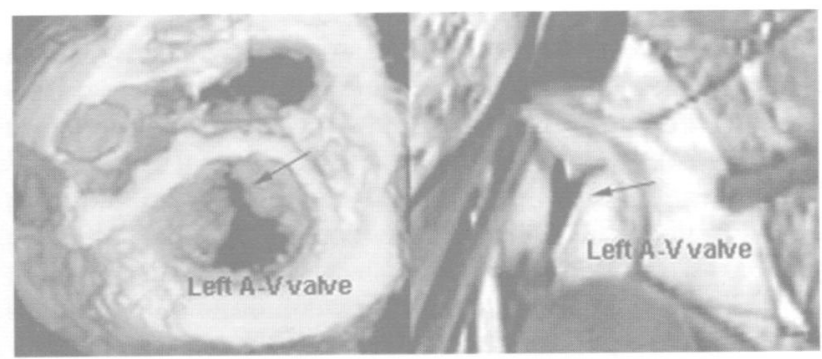

double-orifice LAVV was missed by $3 \mathrm{DE}$. The figure illustrates a $3 \mathrm{D}$ reconstructed image of a left AVV viewed from the left atrium with surgical comparison (arrow: cleft AVV).

Conclusions: 3D reconstructed images allowed precise and accurate dynamic assessment of the anatomy of the AVV as well as insights into the exact mechanisms of atrioventricular valve regurgitation and enhances surgical decision making.

\section{P217}

Corticosteroid treatment during cardiac surgery does not stimulate interleukin-10 release but reduces integrin expression on neutrophils and monocytes

J. Hambsch, A. Tarnok, M. Richter, P. Schneider

Cardiac Center Leipzig, University Leipzig, Leipzig, D

Introduction: Cardiac surgery with cardiopulmonary-bypass induces substantial release of the immunosuppressive cytokine interleukin10. $\mathrm{IL}-10$ has been found to be a key cytokine in propagating immune paralysis and leading to septic shock or multi organ dysfunction syndrome (MODS). A recent study (Surgery 1996; 119:76) indicates that corticosteroid administration elevates IL-10 release more than 10 -fold. Thus, corticosteroid could enhance trauma induced immune paralysis and the risk for shock and MODS.

Study: A prospective study was performed in children (age: 3 to $16 \mathrm{yr}$.) who underwent surgery for atrial septum defect (ASDII, $\mathrm{n}=22, \mathrm{ASDI}, \mathrm{n}=3$ ). 16 of them received intra-operatively Methylprednisolone $(3-16 \mathrm{mg} / \mathrm{kg})$. Surgical data, medication, intra- and post-operative care were not different between the groups. Blood samples were drawn pre-, peri- and post-operatively. Serum concentration of cytokines and soluble adhesion molecules was determined by ELISA. Expression of leukocytes adhesion molecules (LFA-1, Mac-1) was quantified by flowcytometry

Results: Patients who underwent surgery showed significant sequestration of IL-10 with maximum values at the end of surgery (peak: $20-540 \mathrm{pg} / \mathrm{ml}$ ) but no significant difference was found with and without Methylprednisolone. By Methylprednisolone IL-6 release was reduced by $>50 \%$, neopterin, TNF-alpha and histamine release was reduced by $>10 \%$ (all $\mathrm{p}<0.05$ ). Post surgical neutrophil and monocyte count, LFA1 and MAC-1 expression and serum levels of ICAM-1 and L-selectin were significantly down regulated $(\mathrm{p}<0.03)$.

Conclusion: Perioperative administration of methylprednisolone does not elevate IL-10 release but reduces infiltration and adhesives of circulating leukocytes. Therefore, intraoperative treatment with corticosteroids seems not to increase the risk for immune paralysis. Migration of monocytes and neutrophils to the sites of inflammation in part accounts for post perfusion injury. The beneficial effect of corticosteroids might be the reduction of this migration ability. (Supported by the Deutsche Herzstiftung).

\section{P218}

Assessment of right ventricular function in children with HLHS using the Myocardial Performance Index A. Sysa, A. Mazurek-Kula, E. Makoniecka, K. Ostrowska, J. Binikouska, T. Moszura, J.A. Moll, J.J. Moll

Polish Mother's Memorial Hospital Research Institute, Lodz, PL

The function of right ventricle is crucial for children with hypoplastic left heart syndrome. The echocardiographic assessment of right ventricular function is difficult and subjective because traditional indexes are based on geometric assumptions that are frequently invalid in these patients.

The Doppler myocardial performance index is independent of geometric assumptions. It measures the ratio of isovolumic time 
intervals to ventricular ejection time derived from Doppler curves.

The aim of this study was to compare MPI in patients with HLHS pre and postoperatively (after Norwood procedure). The same index was calculated in healthy neonates. Echocardiograms of patients with HLHS before and after Norwood procedure between January 1999 and December 2000 were reviewed. Fifteen normal newborns served as control group. The MPI was calculated from Doppler tracings of right ventricular inflow and outflow. Of the 31 patients with HLHS who underwent Norwood procedure only 12 had adequate studies to allow calculation of MPI pre and postoperatively.

In neonates with HLHS before Norwood procedure RV MPI was high $(0.4890 .05$, mean SD). We observed decrease of RV MPI in postoperative course to 0.4500 .03 but this diminution was statistically insignificant. The significance of the MPI decrease after Norwood procedure in children with HLHS should be confirmed in larger group of patients. Myocardial performance index was significantly lower $(0.3100 .06)$ in healthy neonates than in neonates with HLHS.

Conclusion: The MPI seems to be a very useful and easy to perform index for assessment of right ventricular function in patients with HLHS prior and after operative procedures.

\section{P219}

\section{Realtime Magnetic Resonance (MR) - guided}

interventional closure of the atrial septal defect in the porcine model

R. Grabitz, A. Buecker, E. Spuentrup, F. Freudenthal

Dept. of Pediatric Cardiology, Kiel, $D$

Background: Percutaneous closure of atrial septal defects is usually performed under X-ray in combination with ultrasound guidance. We tested the feasibility to apply realtime Magnetic Resonance guidance for percutaneous closure of atrial septal defect in the porcine model in order to avoid the disadvantage of ionizing radiation.

Methods and results: Real-time MR imaging with radial or spiral $\mathrm{k}$-space filling ( 15 frames per second) on an interventional $1.5 \mathrm{~T}$ high field whole body system was exploited to examine the feasibility for MR-guided closure of patent foramen ovale in 7 piglets weighing about 12 to $16 \mathrm{~kg}$. A specially designed prototype MR-compatible closure device was introduced via the femoral vein. The short bore of the magnet and in-room monitors allowed for visualization and steering of the catheter with the loaded occluder. Catheterization of the left atrium and finally correct placement of the device was possible in all cases. Deployment of the device was depicted by real-time $M R$ and initial misplacement, which occurred in two cases, was easily detected and corrected. Measurement of the septal defect size was possible by inflating a sizing balloon over the patent foramen with an aqueous Gadolinium solution of either $1: 5$ or $1: 20$, yielding either a dark or bright signal intensity balloon.

Conclusion: Real-time MR-guidance of atrial septal defect closure is feasible in our porcine model without use of ionizing radiation.

\section{P220}

Tissue Doppler Imaging shows decreased myocardial velocities in children with Fontan circulation A. Mller, V. Razek, J. Tille, J. Weil

Dep. of Pediatric Cardiology, University-Hospital Hamburg, Hamburg, D

Background: Myocardial failure is an important long term complication in patients with Fontan circulation. Tissue Doppler Imaging (TDI) has proved beneficial for the assessment of left ventricular (LV) systolic and diastolic function. It is unclear whether TDI is helpful in assesssing single ventricle physiology with Fontan circulation.

Methods: 13 patients 8,6 years old (4 to 18 years old) with Fontan circulation were examined, as were 14 control subjects with normal hearts. Function was evaluated with standard echocardiography and tissue Doppler imaging of the right ventricular (RV) and left ventricular (LV) free wall and the septum (IVS); respectively right and left free wall in single ventricle morphology. Myocardial velocities were sampled from apex to base at the AV-valve annulus in apical four chamber view.

Results: The Fontan patients showed no signs of cardiac failure in clinical or standard 2D- or M-Mode Echocardiography. The myocardial peak velocities of the functional single ventricle were significantly decreased in systole compared to $\mathrm{LV}$ in normal hearts (right free wall $=5 \mathrm{~cm} / \mathrm{sec}$ vs. $\mathrm{RV}$ free wall $=8,5 \mathrm{~cm} / \mathrm{sec}, \mathrm{p}<0.01$; left free wall $=5 \mathrm{~cm} / \mathrm{sec}$ vs. $7,7 \mathrm{~cm} / \mathrm{sec}$ in LV, p $<0,025$ ). The diastolic velocities of the AV-annnulus in Fontan patients were significantly lower than those of $\mathrm{MV}$-annulus in normal $\mathrm{LV}$ (right free wall $5,9 \mathrm{~cm} / \mathrm{sec}$ vs. $11,7 \mathrm{~cm} / \mathrm{sec}$ in normal $\mathrm{RV}, \mathrm{p}<0,01$; left free wall $7 \mathrm{~cm} / \mathrm{sec}$ vs. $12,1 \mathrm{~cm} / \mathrm{sec}$ in normal LV and IVS, $\mathrm{p}<0.01)$.

Conclusion: Tissue Doppler imaging shows differences in myocardial function between asymptomatic Fontan patients and healthy controls. Abnormal systolic and diastolic myocardial velocities of the right and left free wall were seen in patients with Fontan circulation. This may develop early after establishing Fontan circulation. Further studies are necessary to determine how these changes can help predicting long-term prognosis in Fontan circulation.

\section{P221}

Ventricular morphology as a risk factor for outcome after the cavopulmonary connection

S. Valen, P.B.G. Spronk, A. Dodge-Khatami, G.B.W.E. Benmink, J.F. Hitchoock, E.J. Meijboom

Wilhelmina Children's Hospital/UMC Utrecht, Utrecht, $\mathrm{NL}$

Introduction: Univentricular heart disease is currently treated by the cavopulmonary connection technique. Conflicting results have been reported concerning the influence of ventricular morphology on survival. This study evaluates the influence of ventricular morphology (dominant left/right ventricle) on survival after a partial and/or total cavopulmonary connection (PCPC/TCPC). Methods: A retrospective analysis was performed on 65 patients after a cavopulmonary connection (PCPC/TCPC) performed between July 1969 and September 2001. The influence of ventricular morphology on survival was determined, recognizing hearts with a dominant left or a dominant right ventricle. Start point of follow-up was date of operation and endpoints were death or takedown of CPC. In addition, we assessed the mean age at time of operation and how this changed over time.

Results: 37 patients were diagnosed to have a dominant left ventricle (male/female ratio $=1,31$ ), 28 a dominant right ventricle $(\mathrm{male} / \mathrm{female}$ ratio $=1.5)$. In these two groups, 8 and 10 patients died, respectively. One patient was lost to follow-up and one patient underwent a CPC-takedown. Mean length of follow up was 5.795 .57 years (range: $0-27.5$ years).

The influence of ventricular morphology on survival was tested in a univariate model in patients treated before January 1996 $(p=0.0911)$, after ' $96(p=0.3199)$ and for the entire cohort $(\mathrm{p}=0.0624)$.

The mean age at time of surgery decreased significantly for: $\mathrm{PCPC}$ in dominant right ventricle patients (from $5 \mathrm{y} 1 \mathrm{~m}$ to $1 \mathrm{y}$; $p=0.044$ ), TCPC in dominant left ventricle patients (from $4 \mathrm{y} 7 \mathrm{~m}$ to 2 y $8 \mathrm{~m} ; \mathrm{p}=0.029)$ and in dominant right ventricle patients (from 8 y $11 \mathrm{~m}$ to $3 \mathrm{y} 11 \mathrm{~m} ; \mathrm{p}=0.023$ ). 
Conclusions: In this study ventricular morphology was not found to be a risk factor for survival. This means that patients with a dominant left ventricle do not have a significantly better outcome after $\mathrm{CPC}$ than patients with a dominant right ventricle. Although for the entire cohort the influence of ventricular morphology approached significancy, it only decreased after 1996. This could be explained by improved surgical techniques and a significant decrease of the mean age at the time of CPC after 1996.

\section{P222}

The role of stress echocardiography in the follow up of patients with Transposition of the great arteries (TGA) after Arterial Switch operation (ASO)

I. Spadoni, M. Terrazzi, G. Kraft, S. Redaelli, M. Carminati, V. Vanini, S. Giusti

Paediatric Cardiology, CNR, G. Pasquinucci Hospital, Massa, I

The diagnostic tools routinely employed in the follow up evaluation of patients (pts) with TGA and ASO are often inadequate to identify myocardial ischemia caused by abnormalities of the coronary arteries. Fifty-five patients with TGA and ASO, mean age 64 years (range 4 months-21 years), mean weight $2515 \mathrm{~kg}$ (range 6-89 kg) underwent Dypiridamole echo and selective coronarography 4 months-18 years (mean 63 years) after operation. Dypiridamole echo was performed under general anesthesia immediately before coronarography and was well tolerated without complications. In 53 patients $(96 \%)$, included 2 cases with ischemic basal ECG and 13 with rest abnormalities of segmental motility, no ischemic regional dyssinergies were induced by Dypiridamole. In these 53 patients no coronary lesions were detected by coronarography. Dypiridamole echo was positive for ischemia in 2 pts (2\%): $1 \mathrm{pt}$ did not show stenosis of the coronary arteries at coronarography; Dypiridamole scintigraphy was then performed confirming segmental perfusion alterations in the same area as Dypiridamole echo, probably caused by microcirculation abnormalities. In the other patient with ischemia induced by Dypiridamole, occlusion of the ostium of the right coronary artery and severe stenosis of the anterior descending were identified.

Dypiridamole echo is a simple and repeatable tool that shows good correlation with coronarography for the diagnosis of coronary abnormalities.

In our opinion stress echocardiography is the method of choice for the evaluation of inducible ischemia in the follow-up of patients with TGA and ASO.

\section{P223}

Increase in left ventricular torsion to shortening ratio in valvular aortic stenosis detected by Magnetic Resonance Tagging

T. Delhaas, J. Kotte, A. van der Toom, G. Snoep, T. Arts

Dept. of Pediatrics, University Hospital Maastricht, Maastricht, NL

The prognosis in asymptomatic severe valvular aortic stenosis (AS) is widely regarded as too good to justify prophylactic surgery, especially in children. However, as soon as symptoms develop, preand early post-operative mortality rise sharply. While left ventricular (LV) hypertrophy is initially an adaptation to normalize wall stress in pressure overload, it leads ultimately to irreversible myocardial fibrosis. If operated on, these patients have incomplete LV recovery. It was hypothesized that even in asymptomatic AS patients, hampered perfusion of the LV subendocardium leads to reduced subendocardial myofiber contraction. The subendocardial to subepicardial contraction imbalance leads to an increase of $\mathrm{LV}$ torsion (difference in rotation of two cross-sections) relative to ejection, and, hence, to an increase of the Torsion to Shortening
Ratio (TSR). We investigated whether the TSR is a useful index to detect LV systolic subendocardial dysfunction in the presymptomatic stadium in AS patients.

Six asymptomatic children with valvular AS were investigated using echo doppler, ECG, exercise test, and Magnetic Resonance Tagging (MRT). Tags applied to two cross-sectional slices of the LV by MRT were followed in time. Wall shortening and torsion were quantified using tag displacements. Patient age ranged from 6 to 17 years, body weight from 28 to $74 \mathrm{~kg}$, and maximum flow velocity (Vmax) over the aortic valve from 2.8 to $4.8 \mathrm{~m} / \mathrm{s}$. All ECGs showed signs of LV hypertrophy. Whereas only one patient showed ischemia related ECG changes during exercise, in all patients TSR was increased $(0.310 .02$; mean SD) as compared to controls $(0.230 .04 ; n=10)$, indicating subendocardial dysfunction. Vmax over the stenotic aortic valve was not related to TSR. In conclusion, TSR as detected using MRT proves to be a sensitive parameter for subendocardial dysfunction in children with asymptomatic valvular AS. With other techniques hitherto used this dysfunction cannot be detected, underscoring that foreknowledge about regular cardiac motion can be used to enhance sensitivity on detecting motion disorders. The result inevitably leads to questions as which TSR value indicates beginning of irreversible subendocardial damage and whether the TSR can be used as a parameter to guide prophylactic surgery in AS patients.

P224 (see Abstract 19)

Phase-contrast magnetic resonance imaging measurement of pulmonary venous blood flow in normal and surgically repaired pulmonary veins

E.R. Valsangiacomo, C. Barrea, C.K. Macgouan, J.S. Smallhom, J.G. Coles, S.J. Yoo

University Children's Hospital Zurich, Cardiology, Zurich, $\mathrm{CH}$

P225 (see Abstract 20)

Cerebral function monitoring patterns during neonatal cardiopulmonary bypass

C.C. Geert, N.E.M. Sins, A.N.J. Schouten, M.C. Toet, P.A. Hutter, N.M. Turner, A. Dodge-Khatami, G.B.W.E. Bennink, E.J. Meijboom Children's Heart Center, Wilhelmina Children's Hospital/UMC, Utrecht, $N L$

P226 (see Abstract 49)

Spiral CT scan and angiographic correlations of stents implanted in the aortic arch

M. Chessa, M. Carminati, G. Butera, R.M. Bini, M. Drago,

F. Voterrani, F. Bedogni, D. Negura

Istituto Policlinico San Donato, S. Donato Milanese (MI), I

P227 (see Abstract 50)

Influence of hypoxaemia on the function of ATP

dependent $\mathrm{K}+$-channels in cardiomyocytes from children with congenital cardiac defect

K. Schumacher, E. Jungling, J.F. Vazquez-Jimenez, G. von Bernuth, M-C. Seghaye, A. Luckhoff

Institute of Physiology, University Aachen, Germany, München, D

\section{P228}

Transcatheter closure of patent ductus arteriosus using the swivel-disk and the plug occluders. Initial clinical applications in children

B.D. Thanopoulos, G.S. Tsaousis, E. Basta, A. Zarayelyan

Dept. Cardiology, "Aghia Sophia" Children's Hospital, Athens, GR

Background: In spite of recent advantages in transcatheter management the occlusion of certain anatomic types of patent ductus 
arteriosus (PDA), especially in infants and small children, remains a challenge. The aim of this study was to report initial clinical experience with transcatheter closure of PDA using the Swivel-Disk (SD) and Plug (P) occluders. Methods. Patient (pt) population was divided in to two groups (Gps): GpI, 8 pts, aged 1 to 2 years with an A type PDA who underwent attempted transcatheter closure using the SD occluder. This device is a modified Amplatzer duct occluder with a very low profile retention disc that can swivel (turn) and adapt itself at different angles of PDA insertion. GpHI, 9 pts, aged 0.5 to 3 years, with a $C$ or E type PDA who underwent attempted transcatheter closure with the $\mathrm{P}$ occluder. The $\mathrm{P}$ occluder is a tubular occluder without retention disc made of Nitinol wire mesh. Both occluders are delivered through a $5 \mathrm{~F}$ to $7 \mathrm{~F}$ delivery sheath. Results: GpI. The mean PDA diameter was $3.81 .2 \mathrm{~mm}$ (range, 2.5 to $5 \mathrm{~mm}$ ). All PDAs but one had acute aortic insertion. The mean device diameter was 6.5 to $1.2 \mathrm{~mm}$ (range, 6 to $8 \mathrm{~mm}$ ). Complete angiographic closure was seen in all pts. In 1 pt repositioning of a misplaced device was possible after its readvancement into the aorta. GpII. The mean PDA diameter was $4.23 .5 \mathrm{~mm}$ (range, 1.5 to $10.5 \mathrm{~mm}$ ). The nean device diameter was $6.81 .9 \mathrm{~mm}$ (range, 4 to $12 \mathrm{~mm}$ ). All devices were placed within the PIDA. Complete angiographic closure was observed in $7 / 8$ pts. Placement of $2(5 \times 5)$ coils was required in order to abolish a residual shunt associated with hemolysis in a pt 5 months old with a very large $(11 \mathrm{~mm})$ type E PIDA. No complications were observed. Conclusions: The SD and the P occluders are important adjuncts for transcatheter closure of certain anatomic types of PDA. Further studies are required to document their efficacy, safety and long-term results.

\section{P229}

Heart function and hemodynamic changes after secundum ASD occlusion with the Amplatzer septal occluder during short and long-term FU

A. Zarayelyan, B. Thanopoulos

Yerevan State Medical Lniversity, Yerevan, AR.M

Objectives: Although transcatheter closure with the use of microdevices has been increasly used in the management of Secundum Atrial Septal Defect (SASD), there are no detailed studies examine the impact of such an intervention to the left and right heart mechanical function, especially during long-term Follow-Up (FU). Our study determined LV, RV and LA Volumes and function in a group of ASD patients from 24 hours to 6 months follow implantation of the Amplatzer Septal Occluder (ASO).

Methods: 62 pts with ASD (age $8.5 \pm 3.8$ years) successfully treated with ASO (device size 12 to $30 \mathrm{~mm}$, mean 19,5 $\pm 3.8 \mathrm{~mm}$ ) were studied with $\mathrm{M}$-mode and 2D EchoCG.

Results: Before ASD occlusion there is a type B paradoxical motion of the IVS due to RV volume overload. This causes decrease of LV end-diastolic and of LV end-systolic diameter and volume. As a result the Stork Volume and Ejection Fraction are decreased. 24 hours after occlusion the paradoxical motion of the IVS disappear. In addition due to the decrease RV overload there is increase of LV end-diastolic $(111,9 \%)$ and decreasing of the LV end-systolic $(87,86 \%)$ diameter and volume. This is result increasing of the SV and EF (168\% and $164 \%$ respectively). All before closure data were considered as equal to $100 \%$ for normalization of the age, weight, height, body surface of the different ages children. After occlusion of the SASD it was significant changes also in the LA function: the Volume of the Fast Filling, Volume of Slow Filling and Volume of Atrial Systole increase comparatively with their initial level $(122,6 \%, 205,4 \%$ and $264,1 \%$ respectively). In carly following SASD closure there is significant decrease of RV initially dilated diameter $(76,37 \%)$. After occlusion of SASD the Mitral
Flow Velocity are significantly increase $(124,1 \%)$ and return to its normal level at the 3 months FU.

Conclusion: Transcatheter device closure of SASD has a positive impact on left and right heart function, which is observed early following SASD closure with ASO. At the FU 3 months all indexes became at the normal age-corresponding level and do not changed in long-term FU.

\section{P230}

Interventional closure of residual interatrial communications after modified Fontan operations

A. Gamillscheg, J.I. Stein, P. Zartner, Ch. Suppan, A. Beitzke

Lniversittsklinik fr Kinder- und Jugenheilkunde, Kardiolog, Graz, A

Introduction: After modified Fontan operations (FO) various communications between the systemic and pulmonary venous returns may cause persistent or increasing postoperative cyanosis. Interventional closure of these right-to-left shunts may be necessary to eliminate hypoxemia and to reduce the risk of paradoxical embolic complications.

Patients and methods: 18 patients (pts) with a mean age of $5,6 \pm 4,1(2,5-17,5)$ years underwent interventional closure of a right-to-left shunt $17,4 \pm 15,8(3-60)$ months after a modified FO. After test balloon occlusion fenestrations were closed in $13 \mathrm{pts}$ using an Amplatzer Septal occluder $(\mathrm{n}=7)$, a Rashkind PDA occluder $(\mathrm{n}=3)$, a CardioSeal umbrella $(\mathrm{n}=1)$ and detachable coils $(n=2)$. Residual leaks at the suture lines between the interatrial patch and the right atrial wall were closed using detachable coils and a Rashkind PDA occluder in 2 and 1 pts, respectively. In 3 pts intracardiac venous collateral channels were closed by means of detachable coils.

Results: The mean aortic oxygen saturation increased from $85 \pm 4,5(70-89) \%$ to $91,4 \pm 2,8(83-95) \%(\mathrm{p}<0,001)$ breathing room air and the mean tunnel pressure rose from $10.7 \pm 1.8$ $(6-14) \mathrm{mmHg}$ to $12,1 \pm 2,4(6-16) \mathrm{mmHg}(\mathrm{p}<0,0(01)$. Calculated Qs decreased from $5,15 \pm 2,1(2,1-11,3) 1 / \mathrm{min} / \mathrm{m} 2$ to $3,6 \pm 1,0$ $(1,8-5,6) 1 / \mathrm{nin} / \mathrm{m} 2(\mathrm{p}<0,001)$. Mixed venous oxygen saturation $(66,4 \pm 7,4 \%$ vs. $65 \pm 7 \%)$ and mean systemic arterial pressure ( $73 \pm 8 \mathrm{mmHg}$ vs. $73 \pm 9 \mathrm{mmHg}$ ) remained unchanged. In $1 \mathrm{pt}$ an additional leak of the tunnel could not be closed because of an increase to more than $18 \mathrm{mmHg}$ of the mean pressure in the lateral tunnel during balloon test occlusion. In 2 pts residual leaks after umbrella and coil occlusion of a fenestration and an additional venous collateral channel were closed by means of coils after 16 and 21 months, respectively. At a follow-up of $42 \pm 23(7-99)$ months mean oxygen saturation measured by pulse oxymetry was $93 \pm 2(90-97) \%$. In 2 pts colour-coded Doppler echocardiography revealed a minimal residual right-to-left shunt. In 2 pts contrast echocardiography demonstrated the additional presence of intrapulmonary fistulas. All pts remained free from device migration, thrombembolic events and hemolysis.

Conclusion: After modified FO various right-to-left shunts between the systemic and pulmonary venous returns can be successfully closed using umbrella devices or coils to eliminate cyanosis and to reduce the risk of paradoxical embolism.

\section{P231}

Creation of an unrestrictive lasting atrial communication with a stent in infants

M. Getwillig, L. Mertens, D. Boshoff, B. EYskens

UZ Leuven, Letwent, B

A new technique is presented to create percutaneously an unrestrictive and lasting atrial communication in small children with complex congenital heart disease. 
Procedure: The atrial septal defect is balloon sized to determine the minimal required size of the stent at first inflation. A P104 stent (Palmaz, J\&J) is hand crimped at the distal end of a short tapered $8 \mathrm{~mm} / 2 \mathrm{~cm}$ Opta balloon. The mounted stent is advanced through a $7 \mathrm{~F}$ Mullins sheat until it straddles the atrial septum; this position is checked with fluoroscopy $30-30$ and TEE. The balloon is mildly inflated to open on both sides of the stent; the stent will flail on both ends; minor shifts in position can be made; the stent auto-centres across the septum. The stent is then fully deployed until $8 \mathrm{~mm}$ (nominal value of the balloon). The balloon is exchanged for a bigger balloon ( 10 or $12 \mathrm{~mm}$ ) to obtain a larger communication. The stent will then become very short $(<4 \mathrm{~mm})$. Patients \& results: This procedure was performed in 2 patients (age 2 and + months) with DORV and complex left heart hypoplasia; in both patients a Rashkind procedure and AP banding had been performed in the neonatal period. Both patients had developed retrograde pulmonary hypertension due to a shrinking ASD. By stenting the atrial septum, the gradient and turbulent flow between both atriae disappeared. The stent retained a very stable position without embolisation; thrombus formation or arrhythmia were not observed during 2-9 months follow-up. At explantation the stent was nicely overgrown by endothelium.

The procedure is technically complex and demanding. The interventionalist must avoid problems such as slippage of the stent on the balloon while advancing through the sheat, pushing the stent backward during expansion (septum primum, small left atrium or pulmonary vein, stent to proximal on balloon) or forward (sheat insufficiently withdrawn, stent to much crimped impeding distal opening of balloon, stent to distal on balloon).

Conclusion: Atrial stent dilation can provide a safe, lasting and unrestrictive atrial communication in infants. It is currently our treatment of choice when a necessary atrial communication becomes too restrictive.

\section{P232}

Device failure of tungsten coils implanted for the occlusion of collateral vessels

M. Peuster, V. Kaese, C. Fink, R. Boekenkamp, H. Bertram, H. Haferkamp, G. Hausdorf

Pedatric Cardiology, University of Goettingen, Goettingen, D

Purpose: To evaluate device failure and elevated serum tungsten levels in patients after implantation of tungsten coils (Mechanical Detachable Spirals (MDS); Balt Extrusion, Montmorency, France). Methods: Retrospective review of all patients with coil-occlusion of collateral vessels, patent ductus arteriosus, hepatic hemangioendothelioma and fistulae implanted between 1996 and 1999. Furthermore, toxicity assays with human cell cultures and material analysis of the MDS coils were performed.

Results: In vitro, human endothelial cells, human vascular smooth muscle cells and human fibroblasts were not adversely affected by elevated tungsten concentrations $(60500 \mathrm{~g} / \mathrm{l})$ in the culture medium. Decreased radiopacity of the coils was observed in 9 of 13 patients who had follow-up fluoroscopy during repeat cardiac catheterization. Repeat angiography of the (previously) coil-occluded vessel was performed in 7 patients, 5 patients had recanalization of the vessel. Serum levels were analyzed 6 to 35 months after the implantation in 8 patients. The mean serum tungsten concentration was $6,43 \mathrm{~g} / \mathrm{l}$ (range $2,0 \mathrm{~g} / \mathrm{l}-14,4 \mathrm{~g} /$; normal value $<0,2 \mathrm{~g} / \mathrm{l}$ )

Conclusion: Tungsten coils may dissolve over time and lead to markedly elevated serum tungsten levels with recanalization of previously occluded vessels. Despite no toxicity was encountered in cell culture assays, the clinical use of MDS coils can no longer be recommended. Thorough follow-up of patients treated with MDS coils is mandatory.
P233

Anterograde catheter interventional therapy for critical neonatal aortic stenosis

M. Peuster, S. Schoof, H. Bertram, C. Fink, R. Boekenkamp, C. Jux, G. Hausdorf

Pediatric Cardiology, University of Goettingen, Goettingen, D

Goal: To evaluate our experience with anterograde balloon dilation in patients with critical aortic stenosis.

Methods: Retrospective review of the angiograms, the hemodynamic measurements and echocardiolograms of all 17 patients treated at our institution between June 1996 and August 2000. The mean age was 12 days, mean body weight was $3,3 \mathrm{~kg}$. One patient had hypoplasia of the left ventricle (HLH), one patient was diagnosed with endocardial fibroelastosis (EFE). Prostaglandin E1 was administered in $7 / 17$ patients. All patients had severe left ventricular dysfunction and critical aortic stenosis as was documented by echocardiography. Inotropic support was necessary in $4 / 17$ patients. Results: In all 17/17 patients dilatation of the critical aortic stenosis was possible using an anterograde transvenous approach. In 16 patients a persistent foramen ovale was used for passage to the left atrium, transseptal puncture was performed in one patient. Angioplasty catheters of $3 \mathrm{~mm}$ to $8 \mathrm{~mm}$ diameters were used, in one patient a double-coronary angioplasty balloon technique was used. Periprocedural mortality was $0 \%$. Periinterventionally 2 patients became asystolic but sinus rhythm was quickly restored with single boluses of adrenalin and atropin. No perforation of the aortic valve occured. Mitral regurgitation did not increase after anterograde balloon dilatation of the aortic valve. The preinterventional mean systolic gradient was $73 \mathrm{mmHg}$. Balloon dilatation reduced the gradient to $37 \mathrm{mmHg}$. In 15 patients aortic regurgitation (AR) after balloon valvoloplasty was $<\mathrm{II}$, one patient had II AR and one patient III AR. Left ventricular function improved significantly in all patients after the dilatation. During the follow-up one patient with HLH died during a Daymus-Kaye-Stansel operation at 6 weeks, the patient with EFE died at 8 months of age.

Conclusion: Balloon dilation of critical aortic stenosis can be effectively and safely performed using an anterograde approach.

\section{P235}

Multiple interventional catheterization for treatment of complex cardiac anomalies

M. Szkutnik, J. Bialkouski, R. Bermudez Canete, J. Kusa,

P. Banaszak, I. Herraiz, J. Baranouski, C. E. Mullins

Silesian Center for Heart Disease, Zabrze, $P L$

Patients and method: 14 pts aged 13,6 (from 0.1 to 63) y with different complex anatomical anomalies findings were treated by at least two interventional catheterization (IC) during one session. There were: 4 pts with atrial serptal defect (ASD), 3 with coexisting valvular pulmonary stenosis (PS) and 1 - with recoarctation of aorta (CoA). In all Amplatzer Atrial Septal Occluder (ASO) were implanted after balloon walulo- or angioplasty (BAP). Out of 3 pts with patent ductus arteriosus (PDA) 2 had coexisting PS and 1- native CoA. Embolization of PDA with coils were applied after BAP. There were also 2 pts with postinfarct VSDs. One had double VSD (residual post surgery) and 1 had a coexisting critical stenosis of right coronary artery (RCA). Postinfarct VSDs were closed with $3 \mathrm{ASO}$ and a coronary stent was implanted to stenosed RCA. In $1 \mathrm{pt}$ with double ASD - 2 ASO were placed. In 1 pt post bidirectional Glenn with coexisting stenosis of left pulmonary artery and SVC-RPA anastomosis - 2 Palmaz stents were implanted. In $4,5 y$ old boy with pulmonary atresia (PA) with VSD and one coexisting MAPCA after surgically induced 
Blalock-Taussig anastomosis (BT) and opening of RVOT- both unnecessary vessels (MAPCA and BT) were embolized by coils. In $13 \mathrm{y}$ old girl with midaortic syndrome and right renal artery (RRA) stenosis - 3 Palmatz stent to stenosed thoracic and abdominal Ao were implanted and BAP of RRA was performed. In a $10 \mathrm{y}$ old cyanosed boy (sat 75\%) with AP with VSD and stenosed MAPCA's (the only source of pulmonary supply) 2 coronary stents were implanted to 2 MAPCA's.

Results: In all, but 1 pts procedures were terminated successfully with good hemodynamic result. Mean follow-up was 20 (from 2 to 54) months. In a $3,5 \mathrm{~kg}$ infant with slightly hypoplastic left ventricle it was impossible to close the ASD with a $5 \mathrm{~mm}$ ASO; nevertheless BAP of coexisting reCoA was successful.

Conclusion: Multiple interventional catheterization of some complex cardiac anomalies are an effective treatment allowing to avoid subsequent catheterizations, hospitalization and vascular trauma.

\section{P236}

Novel application of Amplatzer Atrial Septal - and Duct Occluders

J. Bialkouski, M. Szkutnik, J. Kusa, P. Banaszak, M. Zyla-Frycz, M. Zembala

Silesian Center For Heart Disease, Zabrze, PL

Background: Amplatzer Atrial Septal and Duct Occluders (ASO \& ADO) were applied routinely in our center to close 132 Atrial Septal Defects and 8 wide Patent Ductus Arteriosus. The unique construction of $\mathrm{ASO}$ and $\mathrm{ADO}$ permits embolization of other undesirable shunts, which is sometimes a life saving procedure.

Patients \& methods: Seven ASO were applied to close following defects: post infarct VSD in 5 pts (aged from 51 to 71 -mean $61 \mathrm{y}$ ), huge intrahepatic veno-venous fistula in a $5 \mathrm{y}$ old boy and post Fontan fenestration in $9 \mathrm{y}$ old girl. One ADO was implanted to proximal end of pulmonary arterio-venous (a-v)fistula in a 21 old man. In patients with VSD the procedure was performed between 1.5 and 12 months (mean $4.5 \mathrm{~m}$ ) after myocardial infarction. The stretched diameter of VSD ranged from 15 to 19 (mean 17) mm. One of the VSD pts had 2 residual postop VSD. All were in III/IV NYHA class. Pt with intrahepatic fistula was heavily cyanotic (sat $45-50 \%$ ), fistula's diameter was $20 \mathrm{~mm}$. Fontan fenestration had a $5 \mathrm{~mm}$ - and pulmonary a-v fistula a $10 \mathrm{~mm}$ of diameter. In all pts venous jugular approach was applied.

Results: All procedures but one were finished successfully. In 4 pts post infarct VSDs were closed with $5 \mathrm{ASO}$ (in postsurgical pt 2 ASO were applied) and immediate significant clinical improvement was achieved. The size of implanted devices ranged from 16 to 20 (mean 18) $\mathrm{mm}$. In one pt cannulation of VSD appeared impossible both from venous and arterial side, because of its oblique intraseptal trajectory (which was confirmed during subsequent operation). Both intrahepatic fistula and post Fontan fenestration were successfully closed with $20 \mathrm{~mm}$ and $5 \mathrm{~mm}$ ASO respectively. Congenital pulmonary arteriovenous fistula was embolized with $12 / 10 \mathrm{ADO}$. In the latter 3 cases the right to left shunt was permanently abolished and significant increase of saturation was achieved.

Conclusions: Implantation of Amplatzer occluders is a simple and reliable technique for closure of intra- or extra-cardiac undesirable shunts with low incidence of complication and high success rate.
P237

Long-term follow-up data of neonates with critical pulmonary stenosis and pulmonary atresia with intact ventricular septum

L. Krnyei, S. Millyi, L. Kirly, A. Szatmri

Hungarian Pediatric Heant Center, Budapest, $H$

Objectives: Data of neonates with critical pulmonary stenosis (cPS) or valvular pulmonary atresia, intact ventricular septum (PA) underwent balloon dilatation (BD), surgical valvulotomy (SV), surgical valvulotomy and Blalock-Taussig shunt $(\mathrm{S})(\mathrm{SV}+\mathrm{S})$ were evaluated and compared retrospectively:

Methods: Included: 1. cPS or PA Excluded: 1. hypoplastic/atretic TV 2. RV sinusoids. Period: January 1992-December 2001. 3. patient groups: $\mathrm{BD}, \mathrm{SV}, \mathrm{SV}+\mathrm{S}$. Mortality (M), reintervention rate (RR), reintervention free interval (RFI), and freedom from reintervention (FR) 1, 3 mos, 1, 3 and 6 years were assessed.

Results: Group of $\mathrm{BD}(\mathrm{n}=22): 18$ pts are free from reintervention (mean RFI: 2,6 years, range: $0,1-6,3$ yrs). 1 pt died related to BD. 3 pts required surgery: residual pulmonary stenosis $(n=2$, RFI: $22 ; 47$ days), severe hypoxemia after BD ( $n=1$, RFI: 4 days). 1 of this 3 died at the operation, another needed further reinterventions.

Group of SV $(\mathbf{n}=9): 1 \mathrm{pt}$ is free from reintervention (RFI: 9.1 years). 5 pts died in the early postoperative period. 3 pts needed reoperation for restenosis (RFI: 82, 85, 102 days), 2 of them died during surgery, 1 needed a second reoperation 5 yrs later.

Group of $\mathrm{SV}+\mathrm{S}(\mathrm{n}=7): 2$ pts required $\mathrm{BD}$ for restenosis (RFI: 2.1 and 1,1 yrs). 3 pts required surgery of LPA and closure of the BT shunt. (RFI: 4,8; 5,1; 4.5 years). 1 pt needed both (RFI: 71 days; 1.5 years). 1 pt underwent $\mathrm{BD}$ and coil embolisation of the BT shunt. (RFI: 4.9 years).

\begin{tabular}{lllllllll}
\hline & & & & & & & \\
\cline { 4 - 8 } & & & & & & & \\
& & RR & 1 month & 3 months & 1 year & 3 years & 6 years \\
& $(\%)$ & $(\%)$ & $(\%)$ & $(\%)$ & $(\%)$ & $(\%)$ & $(\%)$ \\
\hline $\mathrm{BD}$ & 4.5 & $14^{\#}$ & 90 & 85 & 85 & 85 & 85 \\
$\mathrm{SV}$ & $56^{\star}$ & 75 & 44 & 22 & 11 & 11 & 11 \\
$\mathrm{SV}+\mathrm{S}$ & 0 & 100 & 100 & 86 & 86 & 57 & 14 \\
\hline
\end{tabular}

* $\mathrm{P}<0,05$ vs group $\mathrm{BD}, \mathrm{SV}+\mathrm{S} .{ }^{*} \mathrm{p}<0,05$ vs group $\mathrm{SV}+\mathrm{S}, \mathrm{SV}$

Conclusions: 1. BD is effective, with good long-term result 2. Mortality of $\mathrm{SV}$ is significantly higher than of $\mathrm{BD}$ or $\mathrm{SV}+\mathrm{S}$ 3. RR was significantly higher in the surgical groups. $4 . \mathrm{SV}+\mathrm{S}$ had $100 \%$ RR

\section{P238}

\section{Clinical history after aortic balloon valvuloplasty}

M. Witsenburg, W.A. Helbing, M. Dalinghaus, R.M.F. Berger

Sophia Children's Hospital/Erasmus MC, Rotterdam, $N L$

Objective: Evaluation of clinical outcome after balloon aortic valvuloplasty (BAV).

Methods: Retrospective analysis of patient files in a tertiary referral center.

Results: Since 1986 BAV was used as primary treatment in all consecutive patients (pts) with severe aortic valve stenosis (VAS) and in pts with residual stenosis after surgery. In a 15 year period 82 pts ( 58 boys, 24 girls, age range 3 days $-15 \mathrm{yrs}$ ) underwent BAV for VAS. $38 / 82$ pts were infants. $18 / 82$ pts had undergone a previous surgical valvotomy. Follow-up (mean 7 yrs) was complete in 80 pts. Mortality concerned 8 pts, of whom 7 during infancy. Two infants died during the intervention, caused by aortic regurgitation in one and arterial bleeding in the other. The other 6 pts died because of 
cardiac failure in 4 , endocarditis in one and acute arrhythmia 11 yrs after BAV in one pt who refused surgery. Re-interventions were necessary in 40 pts during follow-up: repeat BAV in 11 pts, surgical aortic valvulotomy in 3 and valve replacement in 26 pts. The indication for valve replacement (pulmonary autograft) in these 26 pts was valve stenosis in $44 \%$, regurgitation in $33 \%$ and a both stenosis and regurgitation in the remaining. Of the $11 \mathrm{pts}$ that underwent a repeat BAV 4 required subsequent valve replacement during follow-up. At 5 yrs follow-up $53 \%$ of pts were free from any re-intervention. During the study period up endocarditis occurred in 4 pts which was fatal in one.

Conclusion: In infancy mortality and the need for re-interventions after BAV for VAS is considerable. After infancy BAV for VAS is a successful and low risk therapy at long term, postponing surgical intervention in the majority of pts.

\section{P239}

Catheter closure of patent foramen ovale for secondary prevention of tias and strokes: acute results and followup in 471 patients

L. Krumsdorf, K. Billinger, T. Trepels, S. Ostermayer, E. Zadan,

K. Horvath, H. Sievert

Cardiovascular Center Bethanien, Frankfurt, D

Background: Patients with transient ischemic attack (TIA) and stroke related to patent foramen ovale (PFO) are at risk of recurrence. Catheter closure of PFO has been proposed as an alternative to surgical closure and anticoagulation. So far information about the long-term results of this procedure is limited.

Methods: Since August 1994, catheter PFO closure was attempted in 471 patients aged 17 to 80 years (mean 4813 ). The PFO was suspected to have caused 1 to 9 paradoxical embolic events (stroke in 379, TIA in 254, prolonged ischemic neurological deficit in 19 and peripheral embolism in 31 patients). Other sources of embolism were not found. The annual recurrence rate after the first event and before PFO closure was $26 \%$. Balloon sizing of the PFO revealed a diameter between 3 and $21.2 \mathrm{~mm}$.

Results: PFO closure was successfully performed in all patients using 8 different closure devices. In 10 patients a second attempt was necessary; 9 patients received 2 occluders. In 7 patients surgery was performed; 1 patient needed emergeny because of pericardial tamponade and 1 died of endocarditis. During follow-up (0.03 to 71 months, mean 1415$) 13$ clinical events similar to embolic events occurred. Only 2 of these patients had a residual shunt. The annual recurrence rate of events was reduced from $26 \%$ before closure to $2.6 \%$ after closure

Conclusion: PFO closure is a safe and effective technique in preventing paradoxical embolism and may be an alternative to live-long anticoagulation. However, like in any interventional procedure severe complications may occur. Therefore, $\mathrm{PFO}$ closure should be considered investigational until data from randomized trials confirm its therapeutic value

\section{P240}

Advantages and limitations of ASD closure with the Amplatzer-occluder: single center experience in 209 consecutive adult patients

L. Krumsdorf, E. Zadan, K. Horvath, S. Ostermayer, T. Trepels,

K. Billinger, H. Sievert

Cardiovascular Center Bethanien, Frankfurt, D

Background: Atrial septal defect (ASD) closure has been reported as a suitable alternative to surgery in children. This is a report of the results in one of the world largest series of adult patients.
Methods: Between November 1997 and November 2001, catheter closure of an ASD was attempted with the Amplatzer ASD occluder in 209 consecutive adult patients (mean age, 4517 years; range, 13 to 90 ). These patients had an atrial septal defect with a diameter between 3 and $34.5 \mathrm{~mm}$ (21 $5 \mathrm{~mm}$ ). Qp:Qs ranged from 0.8 to $4.2(2.00 .6) .14$ patients had multiple defects. The systolic pulmonary artery pressure was $>30 \mathrm{mmHg}$ in 67 patients (32\%) and $>50 \mathrm{mmHg}$ in 19 patients $(9 \%)$.

Results: The implantation of the occluder was primarily successful in all 209; 188/209 procedures could be performed under local anesthesia. The device diameter varied from $12 \mathrm{~mm}$ to $40 \mathrm{~mm}$ (median $22 \mathrm{~mm}$ ). The mean procedure time was $4620 \mathrm{~min}$., the X-ray time $7.95 .4 \mathrm{~min}$. and the length of hospital stay 0.91 .1 days. Transesophageal echocardiography after implantation, at 2 weeks or at 6 months, showed complete closure of the defect in 204 of 209 patients. A thrombus could be observed in one case An asymptomatic embolization of the device occurred in 1 patient (surgery uneventful), pericardial effusion in 1 (asymptomatic) and air embolism in 1 (without clinical consequences). During follow-up (0.03 to 32 months), one patient developed pericardial effusion (pericardiocentesis successful, no sequelae) and in a second patient an arosion of the aortic root occurred (surgical explantation of the device successful). 9 patients developed atrial fibrillation (cardioversion has been successful in all of them).

Conclusion: Transcatheter ASD closure with the Amplatzer occluder is a reliable and very safe procedure with excellent short and medium term results even in adult patients. High closure rates of up to $98 \%$ can be achieved.

P241

Data of prospective angiographic follow up of patients underwent balloonangioplasty for native aortic coarctation

L. Ablonczy, A. Szatmri

Hungarian Pediatric Heart Center, Budapest, $H$

Background: Follow-up (FU) angiographic data of patients underwent native coarctation balloonangioplasty (NCBAP) is required. Control pressure measurements and angiography (CMA) is one of the "gold standards". Prospective study was performed to determine: 1. the frequency of recoarctation and complications after NCBAP, 2. optimal age for procedure.

Patients and method: Between 1994-2001,92 pts (12 pts $<1$ vear) underwent NCBAP, 43 were eligible for FU CMA, performed electively 24-48 months after NCBAP unless recoarctation was present earlier. Median age at CMA was 9 vears $(6$ months -17 years). Frequency of recoarctation, aneurysm, dissection in relation to the anatomic types were assessed. Aneurysm diameter $>30 \%$ of aortic arch was indicated for surgery. The ratio of ascending aorta/arch diameter (aa/arD) was measured by angiographic software before the intervention and at the time of the CMA

Results: Recoarctation (gradient $>20 \mathrm{mmHg}$ ) was found in $6 \mathrm{pts}$, 5 were $<6$ month age at NCBAP. Insignificant recoarctation (gradient $<20 \mathrm{mmHg}$ ) was in 10 pts. No gradient was found in $27 \mathrm{pts}$. In 14 pts coarctation was membraneous $(M)$. Two patients presented with mild aortic arch hypoplasia (aa/arD:2.20; 2.13 ), both required re-intervention. No change was observed in aa/arD at CMA (aa/arD mean at NCBAP: 1.52 (range: $1.03-2.20$ ), at CMA $1.52(1.07-2.13), \mathrm{p}=\mathrm{ns})$. Aneurysm were found in $10 \mathrm{pts}(23 \%)$, 8 of them older $>4$ years. Anatomic type of coarctation in 6 aneurysm cases were M. Surgery was required in 6 pts. Small dissection was present in $10 \mathrm{pts}(23 \%)$, in 4 together with aneurysm $(9 \%)$. Eight of 10 pts were $>4$ years old.

Conclusion: 1. CMA is useful to follow these pts. 2. Complications appeared mostly in $M$ type of coarctation. 3. Aneurysm and 
dissection are not rare complications after NCBAP. 4. Recoarctation after NCBAP performed at $<6$ months of age is frequent 5. Majority of aneurysms and dissections were found in pts $>4$ years. 6 . Therefore, the ideal age for NCBAP seems to be $>6$ month and $<4$ years of age.

\section{P242}

Transcatheter septal reduction for Hypertrophic Cardiomyopathy in children using radiofrequency energy - a pilot study

J.V. De Giovanni, J. Vettukattil, R.A. Edgar, J. Timmins

Heart Unit, Birmingham Children's Hospital, Birmingham, UK

Hypertrophic Cardiomyopathy (HOCM) is a disorder of amino acid sequence which manifests itself clinically by inappropriate myocardial hypertrophy, supernormal contraction and impaired diastolic compliance. In addition, there is variable, dynamic left ventricular outflow obstruction (LVOTO), mitral regurgitation as well as potential for arrhythmias, some of which can be fatal.

The LVOTO contributes to symptoms including reduced exercise tolerance, angina and syncope. Relief of LVOTO is indicated in the presence of symptoms in association with a significant gradient (arbitrarily taken at $50 \mathrm{mmHg}$ or more). Reduction in LVOTO can be achieved by surgery, pacing or by alcohol injection in a septal coronary artery. The latter is unsuitable for children, and we have look at an alternative transcatheter approach using radiofrequency (RF) energy to create septal lesions in order to reduce septal thickness and hence improve the LVOTO.

Four symptomatic patients with HOCM and significant LVOTO underwent haemodynamic evaluation at rest, following temporary pacing with variable AV delay as well as septal ablation using RF. Several RF lesions were made in the septum at the site of obstruction. These were guided using biplane fluoroscopy and trasoesophageal echocardiography; intracardiac electrograms were recorded to identify the position of the His potential. Cool-tip catheters were used in order to maximize the depth of the lesion. Full heparinisation took place during the procedure followed by oral aspirin.

Complications: One patient developed transient complete heart block and was paced as he showed a reduction in gradient with short AV delay pacing; two others were also paced for short AV delay in the absence of block. One patient had a large haematoma at the site of catheter entry and this resolved spontaneously.

There was no immediate change in the gradient but the Doppler gradient fell by $50 \%$ after $4-6$ weeks following the procedure. One patient is showing an increase in LVOTO 2 years after the original procedure.

Conclusion: Trancatheter septal ablation using RF for significant LVOTO in HOCM is promising as an alternative or adjunct to other treatment modalities. It is feasible in children and may be repeatable in case of recurrence. Further evaluation and follow-up is required.

\section{P243}

Bailout catheter interventions early after cardiac surgery C. Fink, M. Peuster, G. Hausdorf

Pediatric Cardiology, University of Goettingen, Goettingen, $D$

Aim: To report our experience with early postoperative bailout catheter interventional procedures in patients after cardiac surgery. Methods: Retrospective review of all 1048 patients undergoing catheter interventional procedures between April 1997 and October 2000. Patients were included if cardiac surgery had been performed within 14 days prior catheter interventional therapy. Patients: Mean age at interventional cardiac catheterization was 310 days (range 8 days 1584). Diagnoses: Tetralogy of Fallot $(\mathrm{n}=3)$, pulmonary atresia and VSD $(\mathrm{n}=3)$, hypoplastic left heart syndrome $(n=2)$, tricuspid atresia $(n=2)$, coarctation of the aorta $(n=1)$, absent right pulmonary artery and multifocal perfusion of the right lung $(n=1)$, univentricular heart $(n=1)$. Cardiac Surgery: Norwood type operation $(n=4)$, aortopulmonary shunt creation $(n=4)$, unifocalization of the pulmonary circulation ( $\mathrm{n}=2)$, RV-PA homograft $(\mathrm{n}=1)$, coarctation repair $(n=1)$, Fontan operation $(n=1)$. Catheter Interventions: Acute shunt obstructions were treated using balloon angioplasty in 3 patients, in 1 patient, stent implantation was performed. Obstruction of the pulmonary circulation after reconstructive surgery was performed using stent implantation in 3 patients, 2 patients were treated by balloon angioplasty. Pulmonary hypoperfusion was treated by stent implantation into stenotic collaterals after unifocalization in 1 patient. Obstruction of the reconstructed aortic arch was treated by balloon angioplasty in 1 patient. A obstructed Fontan fensetration was recanalized by balloon angioplasty in 1 patient. Gangrene of the right lower extremity after coartation repair was treated by balloon recanalization of the femoral artery.

Results: 12 of 14 interventions were successful and resulted in weaning from the respirator or extracorporeal support. In one patient, balloon angioplasty failed to restore pulmonary perfusion via an aorto-pulmonary shunt and the patient underwent successful surgical revision of the shunt. One patient after Norwood operation and re-coarctation died in cardiac failure despite extracorporeal circulation 4 days post interventionally.

Conclusion: Early postoperative catheter interventional therapy is a safe and effective alternative to surgical revision and is associated with low mortality.

\section{P244}

Transcatheter closure of medium and large size patent ductus arteriosus with the Amplatzer PDA Occcluding device: Short-term and medium-term results

F.F. Pinto, C. Trigo, G. Nogueira, L. Sousa, A. Agapito,

A.M. Antunes, S. Kaku

Hospital de Santa Marta - Servio de Cardiologia Peditrica, Lisboa, P

Purpose: The aim of this study was to assess the effectiveness of transcatheter occlusion of medium and large size patent ductus arteriosus (PDA) with the Amplatzer PDA Occluder (A-PDA-O), through the results of immediate and medium term follow-up. Introdution: Occlusion of Medium and large size PDA (diameter $>2 \mathrm{~mm}$ ) has been performed with several devices, with variable degrees of success and residual shunt ranging from 3 to $38 \%$. The A-PDA-O seems to be a safe and more effective device for this purpose.

Methods: For a period of three years, from November 1998 until December 2001, 20 patients (pts) were selected for PDA occlusion with the A-PDA-O. Group I: consisted of 16 pts with a mean age of 4,25 years and a mean weight of $14,5 \mathrm{Kg}$; Group II: consisted of 4 adult pts with a mean age of 41,25 years. Morphologically the most frequent PDA type was A-1, in 11 pts; 7 pts had A-1 type and 2 had $C$ type. The mean PDA diameter was $3,75 \mathrm{~mm}$ for Group I and $3,1 \mathrm{~mm}$ for Group II. Pts were evaluated immediately by angiography and at 24 hours, 6 and 12 months later clinically and by echocardiography looking for residual shunt and complications. Results: All selected pts were successfully occluded with the A-PDA-O. There were no immediate complications. Immediate angiographic results demonstrated complete occlusion in $50 \%$ pts for both Groups. Echocardiographic evaluation at 24 hours revealed an occlusion rate of $75 \%$. All pts were dismissed home the next day. At 6 months the occlusion rate was $100 \%$ evaluated by echocardiography. For a follow-up period of 13,5 months for 
Group I and 23 months for Group II, all pts are symptom free, with no medication, nor late complications such as stenosis at pulmonary arteries (PA mean velocity: Group $\mathrm{I}=1,12 \mathrm{~m} / \mathrm{seg}$; Group II $=1,11 \mathrm{~m} / \mathrm{seg}$ ) or descending aorta (Ao mean velocity: = Group I = 1,21 m/seg; Group II = 1,17 m/seg).

Conclusions: Transcatheter occlusion of medium and large size PDA using the A-PDA-O, seems to be a very safe and effective therapy both at immediately and medium-term follow-up.

\section{P245}

\section{Long-term follow-up after balloon valvuloplasty for} critical aortic stenosis

H.P. Gildein, G. Balling, A. Eicken, Th. Genz, W. Sebening, J. Hess

Deutsches Herzzentrum München, München, $D$

Since 1983 balloon valvuloplasty (BV) is available as nonsurgical treatment for critical aortic stenosis (AS). However, there is little information on medium- to long-term outcome in large study groups from single centres. We therefore looked at the outcome and follow-up treatment of all survivors with a potential followup period of at least 5 years after BV.

From March 1986 when BV of critical AS was initiated at the German Heart Centre until April 199739 neonates with critical AS underwent BV. There were 29 early survivors who formed the study group. Late mortality was $14 \%$ (4 of 29 patients). Two children died at home at the age of 3 months and 14 years. A 22-month-old boy could not be resuscitated from cardiac arrest when he was hospitalised for heart failure. Finally, a 7-month-old infant died after surgical relief of subvalvar mitral stenosis.

In the 25 surviving patients further interventions on the aortic valve were required in 9 children, 4 of whom had repeat BV between the ages of 2 months and 2 years, and in 2 patients surgical commissurotomy was performed as third procedure. In 5 cases a surgical intervention followed BV between the ages of 2 months and 1 year. Three children had mechanical aortic valve replacement, and in 2 children a Ross procedure was performed. Mitral valve surgery was necessary in 4 of the 9 patients with reintervention. Sixteen patients $(64 \%)$ remained free from reintervention after a follow-up period of 10539 months. The maximal Doppler gradient across the aortic valve was 24 to $62 \mathrm{mmHg}$ (mean 40 $14 \mathrm{mmHg}$ ). Only 3 children had aortic regurgitation > grade 2 on colour Doppler sonography.

In conclusion, BV of critical AS provides good long-term outcome with freedom from reintervention in $2 / 3$ of survivors at a mean follow-up period of 8 years. If surgery of the aortic valve is required replacement of the valve either with a mechanical prosthesis or with an autograft may be necessary in most cases. Concomitant mitral valve pathology may be present requiring surgical intervention.

\section{P246}

Stenting native coarctation of aorta in adolescents and adults: experience from a developing country

M. Sadiq, N.H. Malick, Z. Akram, J.V.D. Giovanni, S.A. Qureshi Punjab Institute of Cardiology \& Childrens' Hospital, Lahore, Pak

Background: In developing countries like Pakistan, significant number of adolescents and adults are diagnosed as having coarctation of aorta as diagnosis is often missed in early life. The traditional approach to such pts has been surgery and various techniques have been used to resect or patch it.

Objective: To assess the early and mid-term results of stenting as primary treatment in these pts.

Setting: A tertiary referral center for paediatric \& adult cardiology.
Patients \& Methods: We implanted Cheatham Platinum (CP) stents from NuMED with BIB balloon in 10 adolescents and adults with native coarctation from October 1999 to December 2001. All procedures were done under general anaesthesia. There were 6 males and 4 females, two of them real sisters. The median age was 24 years (range 16-40 yrs). The indication of intervention was systemic hypertension in all pts.

Results: The pre-stent systolic gradient was $50 \mathrm{mmHg}(40-71)$ and disappeared in all patients $(p<0.001)$, except one where a further dilation is planned 6 months later. The mean diameter at the narrowest point increased from mean of $5 \mathrm{~mm}$ to $19 \mathrm{~mm}(p<0.001)$ No vascular complications or thromboembolism occurred Transient abdominal pain and discomfort was observed in all patients. During a median follow up of 1.3 years $(0.1-2.3 \mathrm{yrs})$, none of the nine pts where complete relief was achieved has shown recoarctation on echocardiography. No adverse systemic hypertension has been noticed and 5 of the 9 patients are still on antihypertensive treatment.

Conclusions: Stent implantation in coarctation of aorta in adolescents and adults is an attractive alternative to surgery. Use of $\mathrm{CP}$ stents with BIB balloon allows a better control over site of placement and better stability. It achieves complete relief of obstruction with minimal complications and good intermediate term results.

\section{P247}

Matching equipment to anatomy: Preshaping the long sheath to close very large atrial septal defects

G. Butera, M. Chessa, R. Margherita Bini, D. Negura, M. Carminati Istituto Policlinico San Donato, S. Donato Milanese (MI), I

Background: Percutaneous closure of ASD is now widely used in clinical practice. However, size of the defect and/or deficiency of "rims" around the defect may be responsible for failure. Three hundred and forty-seven consecutive patients with an ASD were found suitable for percutaneous occlusion with an Amplatzer device. Of these 347 subjects, 131 had a stretched diameter $>24 \mathrm{~mm}$. Some pediatric patients had defects $<24 \mathrm{~mm}$ but these ASDs were very large if compared to the lenght of the interatrial septum. In four out of 131 , we failed the correct position of the device accross the defect using the standard techniques.

Methods: In patients with large ASD, when standard techniques failed we opened the ASO from the mouth of the right upper pulmonary vein. In four patients, due to absent or very deficient rims associated with the large size of the defect did not allow to position the device correctly by using these techniques. In order to achieve a position of the distal disc more perpendicular to the plane of the atrial septum we modified the technique as following: before inserting the long sheath into the body we gave a double curvature, $S$-shaped at the distal $10-15 \mathrm{~cm}$ of it. The first curvature was along the longitudinal axis of the long sheath; the second one was perpendicular to the first. Then, we proceeded in the stantard fashion. Results: By using the technique described above, complete occlusion of the ASD was obtained in four patients in whom standard techniques failed. After having attempted longtime using the standard techniques closure was rapidly obtained with this new technique $(6010 \mathrm{~min}$ vs. $123 \mathrm{~min})$. Neither complication nor residual shunt occurred. Mean follow-up was 3.8 months (from 2 to 5 months) and was uneventful.

Conclusions: A new expedient for very large and "difficult" ASD closure was successfully used in 4 patients in whom standard techniques failed. In these patients perfect closure was obtained modifying the long-sheath in order to obtain a more suitable alignement of the sheath with the atrial septum. Probably, having pre-shaped commercially available long sheaths should be desirable. 


\section{P248}

Adjustable, transvascular pulmonary artery banding with stabilized balloon catheters in swines

P. Zarmer, A. Gamillscheg, M. Zink, A. Beitzke

Department of Paediatric Cardiology, University of Graz, Graz, A

Surgical pulmonary artery banding (sPAB) is palliatively used to reduce pulmonary blood flow and intrapulmonary pressure. In some centres it is also employed in the training phase of the left ventricle before late switch operation. An adjustable banding mechanism and a less invasive procedure might support the patients outcome. Method: We report the results of eight swines (10 to $22 \mathrm{~kg}$ bodyweight) in which different balloon catheters (Baxter Swan Ganz $(\mathrm{n}=1)$, SIMS $(\mathrm{n}=1)$, Corodyn $(\mathrm{n}=4)$ and Tyshak II $(\mathrm{n}=2))$ were implanted into the pulmonary trunc to rise the right ventricular (RV) pressure. The catheter was introduced through the jugular vein, stabilized by a guide wire to avoid dislocation and reduce interference with the valves and fixed in the neck. Balloon diameter, RV pressure and transpulmonary pressure gradient were measured before and after balloon inflation by transesophageal echocardiography (TEE) and invasive pressure measurement. RV pressure was elevated to a mean of $39 \mathrm{mmHg}$ ( $\mathrm{n}=8$, std. dev. $6 \mathrm{mmHg}$, native RV pressure $25 \mathrm{mmHg}$ in mean, std. dev. $3 \mathrm{mmHg}$ ). Balloon filling was controlled daily, heparine and antibiotics administered. After one week measurements were repeated and angiography was performed. Then heart and lungs were explanted. Results: TEE showed a small pulmonary regurgitation, no relevant influence on tricuspid valve function and unaltered contractility. Angiography demonstrated symmetric lung perfusion and no vascular stenosis at the banding site. Elevated RV pressure was found in 4 pigs with inflated balloons $(\mathrm{p}=37 \mathrm{mmHg}$, std. dev. $10 \mathrm{mmHg}$ ), which went to normal values after balloon deflation. Two balloons ruptured during reinflation, but stayed in place. In all pigs a thin precipitating thrombus was found around the balloon. There was no thrombus found in the pulmonary arteries and around the guide wire. A slight myocardial dilation was found in 2 pigs.

Conclusion: With a stabilized balloon in the pulmonary trunc it is possible to achieve an hemodynamic gradient, which is adjustable according to echocardiographic or invasive measurements to the individual growth. Further investigations may prospectively help to replace $\mathrm{SAB}$ and possibly save some palliative surgery to our patients.

\section{P249}

Catheter closure of patent foramen ovale: world experience with the new helex occluder

U. Krumsdorf, N. Wilson, H. Sievert, T. Trepels, W.S. Hillis,

K. Billinger, E. Zadan, A. Salmon, M. Schneider, J. Hess, W. Sebening,

E. Zahn, P. Ewert, S. Qureshi, M. Carminati, J. De Giovanni,

F. Maymone-Martins, L. Latson

Cardiovascular Center Bethanien, Frankfurt, $D$

Background: Transcatheter closure of PFO has been proposed to prevent recurrences in patients with cryptogenic embolic events. We used the new Helex (W.L. Gore, Flagstaff, AZ) occluder for this purpose.

Methods: The Helex Septal Occluder is composed of 2 discs of expanded polytetrafluoroethylene (ePTEE) mounted on a nitinol wire frame. The device is introduced via a $9 \mathrm{~F}$ catheter avoiding the prior use of a long sheath and can be retrieved into the delivery system even after complete deployment. The device is very flexible and has a very low profile once deployed. Transcatheter PFO closure was attempted in 128 patients. The age ranged from 15 to 80 years (mean 5012 ). 175 embolic events occurred in these patients prior to PFO closure. The diameter of the PFO measured with a balloon catheter ranged from 3 to $23 \mathrm{~mm}$.

Results: PFO closure was successfully performed in all patients. The device diameter was $15 \mathrm{~mm}$ in $36,20 \mathrm{~mm}$ in $53,25 \mathrm{~mm}$ in 29 , $30 \mathrm{~mm}$ in 9 and $35 \mathrm{~mm}$ in 1 patient. $108 / 128$ procedures could be performed in local anesthesia. Mean X-ray time was $10 \mathrm{~min}$. including balloon sizing of the defect. Transesophageal echocardiogram (TEE) showed a residual shunt in 38 patients. One device embolized some hours after implantation to the aortic bifurcation (asymtomatic) and was easily retrieved with a snare. A larger Helex was implanted successfully. After 6 months $(n=72)$, only 8 patients had a residual shunt. During follow-up ( 0.03 to 23 months) no further embolic events and no other complications occurred.

Conclusion: PFO closure with the Helex occluder is a very safe procedure and prevents further embolic events. Advantages of this device compared with other devices are soft atraumatic edges, lack of risk for air embolism, complete removal at any stage during or after deployment, low thrombogenity, and high rate of complete closure at follow-up.

\section{P250}

Long-term results after aortic balloon dilation in neonates and infants

S. Giusti, I. Spadoni, G. Kraft, M. Carminati

Paediatric Cardiology, CNR, G. Pasquinucci Hospital, Massa, I

The late outcome of 32 patients undergoing consecutive successful aortic balloon dilation during the first year of life was examined. The mean age at the procedure was 86 days (range 2-365) and the mean weight $4.8 \mathrm{~kg}$ (range 2.1-10). Fifteen patients were in critical conditions before the balloon dilation and 5 had associated aortic coarctation. The valvuloplasty produced a gradient reduction of $67 \%$ (range $30-100 \%$ ) with a residual peak to peak gradient more than $30 \mathrm{mmHg}$ in 7 patients, mild aortic insufficiency in 11 and moderate in 5. The mean period of follow up was 6.9 years (range $1.2-14.4)$. Seven patients (24\%) developed early restenosis: 4 underwent repeated balloon dilation with good result, 2 surgical valvotomy with significant residual gradient and, in one case, severe insufficiency; in one case the restenosis was associated with moderate regurgitation and the patient underwent Ross operation 5 years after the balloon dilation. Long term follow-up showed residual gradient more than $50 \mathrm{mmHg}$ in 9 patients (31\%), moderate/severe aortic regurgitation in $7(24 \%)$ and mild aortic regurgitation in 13 . During the follow-up period 4 patients (14\%) underwent successfull Ross operation: 3 for stenosis and incompetence of the valve (one of them after surgical valvuloplasty) and 1 for severe incompetence. One patient with progressive increase of the gradient underwent successful balloon dilation 5 years after the first procedure. The other 25 patients $(86 \%)$ are doing well. Long term results of balloon dilation of severe aortic stenosis show a good quality of life without any other procedure in more than $70 \%$ of patients. In our series there is no late mortality. A second procedure (balloon dilation, surgical valvoplasty, Ross operation) was necessary in 8 patients (25\%). Balloon dilation of aortic valve is the more effective and safe palliative procedure for aortic stenosis, with excellent long term results, and remains the technique of choice for the conservative treatment of the aortic valve.

P251 (see abstract 45)

The new intratherapeutics doublestrut stent: in-vitro evaluation of stent geometry following overdilation and initial clinical experience in congenital heart disease F. Ing, J. Mathetwson, M. Cocalis, S. Kirkpatrick, K. Maginot, J. Perry Children's Hospital of San Diego, San Diego, USA 
P252

Implantation of large Amplatzer devices to close atrial septal defects in children: is it a safe practice?

M.J. Raboisson, M. Bourdages, E. Piette, P. De Guise, M. Brassard, J. Mir Hôpital Sainte Justine, Montral, CDN

Background and Objectives: Concerns have been expressed that devices with large disc diameters and profile, such as the Amplatzer, might distort, obstruct or compress various vital structures. These concerns are particularly justified in children, in whom large devices might interfere with the normal growth of structures such as the $\mathrm{A}-\mathrm{V}$ annuli or the aortic root. We thus aimed at verifying the short and mid-term tolerance of large Amplatzer devices implanted in patients with uncompleted growth.

Population and Method: From 12/97 to 06/01, 80 patients $<12$ yrs underwent percutaneous closure of an ASD with the Amplatzer device. The 20 patients with the largest devices (size of left atrial disc/size of patient $0,0370,003$ ) were reviewed clinically, echocardiographically and electrocardiographically. Mean age, weight and $\mathrm{Qp} / \mathrm{Qs}$ were 4,681,98yrs, $15,23,6 \mathrm{~kg}$ and 2,71,0 respectively. The ASD diameter and device size were $17,44.5 \mathrm{~mm}$ and 23,4 3,3 mm.

Results: No procedural complication was observed. The short term follow-up was complicated by $\mathrm{A}-\mathrm{V}$ conduction disturbances $(\mathrm{n}=3)$, pericardial effusion $(\mathrm{n}=5)$ and moderate mitral regurgitation $(\mathrm{n}=1)$. All these complications produced no symptoms and spontaneously recovered by one month. At last follow-up $(13,210,2$ months) there was no case of increased valvar regurgitation (compared to pre-implantation), systemic or pulmonary venous obstruction, significant rhythm or conduction disturbances, progressive aortic root distortion or any other complication. At immediate follow-up, proto-systolic contact between the left disc and the anterior mitral leaflet and straddling of the aortic root by the device were respectively observed in $50 \%$ and $60 \%$ of patients. By last follow-up, the prevalence of such anomalies was $20 \%$ and $45 \%$, while the mean distance between the left atrial disc and the plane of the mitral annulus increased from $0,92,2 \mathrm{~mm}$ to $2,12,0 \mathrm{~mm}(\mathrm{p}<0,01)$ and the amount of aortic straddling decreased from $2,12,6 \mathrm{~mm}$ to $0,82,3 \mathrm{~mm}$ ( $\mathrm{p}<0,01$ ).

Results: Large Amplatzer devices produce an increased amount of immediate complications, which were all asymptomatic and spontaneously recovered in our series. These devices do not seem to cause mid-term complications or progressive distortion of cardiac structures, even in a population of growing children, confirming the safety of this practice.

P253 (see Abstract 52)

Interventional catheterization procedures in the treatment of hypoplastic left heart syndrome

A. Lebing, G. Fischer, J. Stieh, J. Scheewe, A. Boening, H.H. Kramer

Department of Paediatric Cardiology, University of Kiel, Kiel, D

\section{P254}

Transcatheter occlusion of perimembranous ventricular septal defect with standard Amplatzer device

R.M. Bini, M. Chessa, G. Butera, A. Cazzaniga, G. Isgr, M. Ranuci, M. Carminati

Istituto Policlinico San Donato, S. Donato Milanese (MI), I

Background: Among the most recent accomplishment in the field of interventional cardiology, transcatheter closure of ventricular septal defects (VSD) is still under discussion. The Amplatzer VSD
Occluder (ASO-VASD), a double umbrella of nitinol structure with a Dacron mesh, available in sizes from 6 to $24 \mathrm{~mm}$, has made this procedure simpler and less risky. More and more centers are using the ASDO-VSD for occlusion of congenital or acquired (post infarction) muscular VSDs.

Methods and results: We have used the standard ASO-VASD in 7 patients, 2 males, with perimembranous VSD, usually considered a contraindication for the transcatheter approach, due to the proximity of the defect to the aortic valve. Patients age was: 5, 6, 8,9, 25,30 and 63 years. One patient had a coarctation of the aorta treated simultaneously by stent implantation. The selection of patients was made on 2-D echo and no patients considered suitable on 2-D echo was discarded on angiography, proving that 2-D echo is a reliable method to select patients. The procedure was carried out through the aorta without the need for an arterovenous circuit. The mean procedural and fluoroscopy time was 49 and 32 minutes respectively. There were no early nor short term ( 6 months) complications. The occlusion was complete without residual shunt in all 5 patients, 2 patients had a small residual shunt and mild aortic insufficiency was detected in 2.

Conclusions: Based on our initial experience select patients with perimembranous VSD may undergo transcatheter treatment with very low risk and good short term results. More data are necessary to compare results of transcatheter techniques with those of surgical closure or with those of natural history.

\section{P255}

Stenting the arterial duct in neonates

M. Gewillig, D. Boshoff, B. Eyskens, L. Mertens

Kindercardiologie, Leuven, $B$

3 neonates $(2,6 \mathrm{~kg}, 3,2 \mathrm{~kg}$ and $3,7 \mathrm{~kg})$ with hypoplastic right ventricle and critical pulmonary stenosis/atresia were treated with radiofrequency ablation and/or balloon dilation. Additional pulmonary flow was required; the duct was therefore stented transvenously (5F sheath; stent: $4 \mathrm{~mm}$, length $1821 \mathrm{~mm}$ Multi-link Tetra Stent). All patients entered the cathlab on IV prostaglandin; the infusion was stopped when the duct was crossed with a 0.014 wire; the stent was implanted after some ductal constriction $45 \mathrm{~min}$ later. Ideally 1 stent must cover the complete duct; the critical marker is the distal duct-aortic junction where the stent should not protrude into the aorta. The proximal end of the stent extended slightly into the pulmonary artery; at no point during follow-up this caused distortion or stenosis of the branch pulmonary arteries. $4 \mathrm{~mm}$ stents in neonates are slightly oversized and allowed generous pulmonary flow; some anti-failure medication was required for 2 months.

Invasive re-evaluation was performed in all 3 patients after 5 months. The pulmonary arteries had grown very nicely without any distortion; vascular capacitance and resistance were normal. The aortic lumen was not affected by the ductal stent. Significant peel formation had reduced the shunt size despite low dose acetylsalicylate $1 \mathrm{mg} / \mathrm{kg} / \mathrm{d}$. In 1 patient adequate right ventricular growth had occurred, making the stented duct redundant; in $2 / 3$ patients reintervention was needed after 5 months because of cyanosis (redilation of stent, followed by a Glenn shunt; the stent could easily be occluded surgically by external compression).

Conclusion: With current technology stenting the duct is a safe and efficient alternative to a surgical shunt, allowing for shorter hospitalisation, no distortion of the pulmonary arteries and ideal pulmonary flow (and resistance) until a Glenn shunt is needed, or right ventricular stroke volume has sufficiently increased. 


\section{P256}

Atrial morphology in hearts with congenitally corrected transposition: implications for ablationists

R. Juneja, E. Rowland, S. Y. Ho

National Heart \& Lung Institute, Imperial College, London, UK

Objective: In view of the possible need for septal puncture to access the left atrium and the occasional difficulty in cannulating the coronary sinus (CS), we investigated relevant anatomical features in the right atrium of hearts with congenitally corrected transposition (ccTGA).

Methods: Nine hearts with ccTGA in the setting of usual atrial arrangement and intact atrial septum were compared with 8 weightmatched normal hearts. All hearts were examined by studying the "septal" aspect of the right atrium with reference to the oval fossa. The anterior margin (A) was arbitrarily measured as the shortest distance from the oval fossa to the superior mitral/tricuspid annulus. The posterior margin $(\mathrm{P})$ was measured from the oval fossa to the posterior-most edge of the right atrial "septal" surface. The total "septal" surface width (W) was measured at the middle of the oval fossa. The dimensions of the stretched oval fossa and the CS isthmus were noted. Mann-Whitney test was used to compare absolute and indexed dimensions i.e. normalized to total width.

Results: The posterior margin in hearts with ccTGA was shorter than in controls $(6.32 .4 \mathrm{~mm}, 111.9 \mathrm{~mm}, \mathrm{p}<0.001$, normalized margin $\mathrm{p}=0.09$ ). The CS isthmus was also significantly shorter $(5.32 .7 \mathrm{~mm}, 11.42 .2 \mathrm{~mm}, \mathrm{p}<0.001)$. In two hearts with ccTGA the CS opening into right atrium was on the same side of the eustachian valve as the inferior caval vein.

Conclusions: The shorter posterior "septal" margin in hearts with cTGA may increase the risk of exiting the heart while performing septal puncture when pointing the needle posteriorly. The shorter CS isthmus and the abnormal location of CS opening in some of these hearts are important when contemplating radiofrequency ablation in this area.

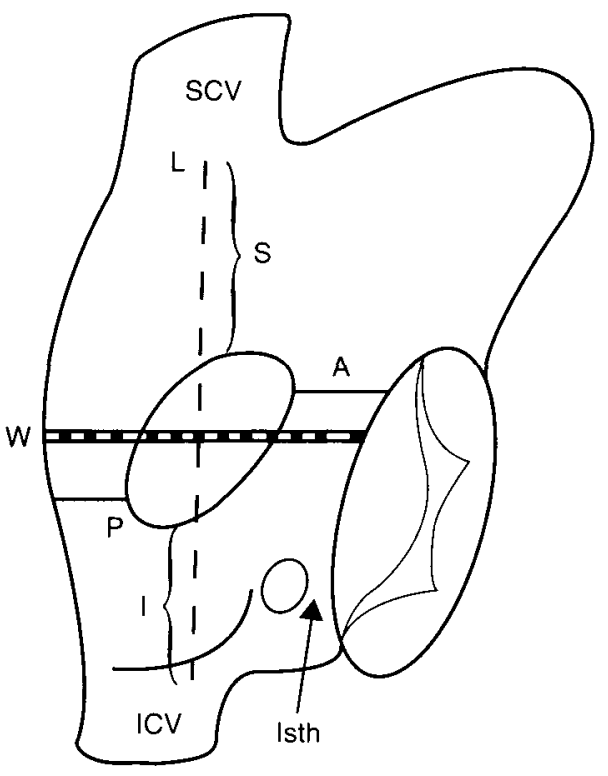

P257

Solid dilators to expand the treatment range of cuttingballoons when enlarging pressure resistant MAPCAs

M. Gewillig, L. Mertens, D. Boshoff

Kindercardiologie, Lewven, $B$

Major Aorta Pulmonary Collateral Arteries MAPCAs may have hypoplastic segments with very narrow stenosis, resulting in profound hypoperfusion of distal lung segments. Frequently these lesions are beyond surgical reach. Some lesions can be treated with balloon dilation and/or stent implantation only; however some lesions are resistant to dilation with pressures up to 25 atmospheres. Moreover, the use of such pressures in balloons frequently results in aneurysm formation or disruption of the adjacent vessel wall.

The Cutting BalloonTM (Boston Scientific, USA) has been shown to give safe and adequate relief of stenosis in MAPCAs; however the cutting-balloons are available only up to $4 \mathrm{~mm}$ diameter, which is small for MAPCAs perfusing many lung segments, especially in adolescents and adults. When a MAPCA is predilated with a $4 \mathrm{~mm}$ cutting balloon, stents frequently cannot be expanded beyond this size.

We therefore attempted to broaden the treatment range by adding 1 or 2 dilators 6-10 French (Mullins sheat, COOK) side-by-side of the cutting-balloon. A total of 5 stenosis in 3 patients were dilated (age, and). An additional vascular access was obtained in all patients (contralateral femoral artery or vein, transjugular or transhepatic) in order to limit vascular damage. The residual stenosis was easily dilated by inflating a $4.0 \mathrm{~mm}$ cutting-balloon TM, allowing final dilation or stenting up to $7 \mathrm{~mm}$. There were no adverse effects, except for transient tricuspid regurgitation while crossing the tricuspid valve with multiple catheters $\&$ sheaths

We conclude that adding solid dilators side-by-side of a cuttingballoon significantly enlarges the clinical spectrum of MAPCAs that can be treated percutaneously successfully.

\section{P258}

Implantation of flow reducing stents for treatment of pulmonary hypertension

I. Michel-Behnke, M. Schneider, M. Sigler, H. Akintwerk,

D. Scitranz

Pediatric Heart Center, Justus-Liebig University, Giessen,

Giessen, $D$

Background: Surgical bilateral pulmonary artery banding in congenital heart disease needs experience, and often results in too much or too less pulmonary blood flow. We describe a new interventional technique for lowering pulmonary artery pressure (PAP) by insertion of newly created flow reducing stents in a lamb model of pulmonary hypertension $(\mathrm{PH})$.

Method: In 6 newborn lambs $(\mathrm{m}=5,5 \mathrm{~kg}$ ) the arterial duct (DA) was stented by implantation of balloon expandable Jo-Stents, to a mean diameter of $7 \mathrm{~mm}$. In the same or a second procedure 1-4 days later, flow reducing stents with a diameter of $10 \mathrm{~mm}$ and a fenestration of $2 \mathrm{~mm}$ were inserted transvenously into the left or the right or both main pulmonary artery branches. PAP was registered before and after ductal stenting. Re-catherization was accomplished 46 weeks later and PAP in the pulmonary artery trunc and distal to the flow reducing stent was checked again. The lambs were sacrificed after the catherization and histological work up was performed.

Results: Ductal stenting resulted in PH in every studied animal at systemic or at least $60 \%$ of the systemic level. There was one procedural death due to arrhythmia, another lamb died, unexplained by the transcatheter procedure. The 4 surviving animals showed mild signs of congestive heart failure. Mean PAP decreased to $m=15-18 \mathrm{mmHg}$ distal to the flow reducing stents. Removal of the stents could be performed with only minor damage to the vessel wall. Histological work up showed endothelialization as well as an inflammatory reaction.

Conclusion: Ductal stenting is a reliable model of pulmonary hypertension in lambs. The new flow reducing stents allow predictable reduction of PAP in flow and pressure mediated PH. The 
method may be applied to human use, where excessive blood flow may induce an increase in pulmonary artery resistance. Easy stent extraction offers the possibility of stent removal during further steps of palliative or corrective surgery

\section{P259}

Long-term follow-up 12 months after implantation of a biodegradable iron-zinc hybrid coil into the subclavian artery of New Zealand white rabbits

M. Peuster, K. Seidler, C. Fink, M. Ehlerding, G. Hausdorf,

C. von Schnakenburg

Pedatric Cardiology, University of Goettingen, Goettingen, $D$

Objective: This study was conducted to evaluate the safety of a biodegradable iron/zinc hybrid coil in a rabbit model.

Methods: 30 New Zealand white rabbits. A femoral arterial cutdown and angiography of the subclavian artery was performed. In 15 rabbits conventional MWCE coils (Cook, Bjaeverskov, Denmark) were implanted, 15 rabbits received one to four hybrid iron/zinc coils. Residual patency of the vessel was documented by selective angiography. Blood samples were drawn before and 3, 6 and 12 months after the implantation. The rabbits were followed for $3(n=9), 6(n=9)$ and 12 months $(n=9)$.

Results: The primary occlusion rate was $100 \%$ for the MWCE coils, and $47 \%$ for the $\mathrm{Fe} / \mathrm{Zn}$ hybrid coils. 1 rabbit in the MWCE group died due to pneumonia, 1 rabbit in the hybrid coil group died early postoperatively due to peritonitis, another rabbit of the hybrid coil group was sacrificed prematurely due to progressive palsy of the upper extremity. There was patency of the coil-laden subclavian artery at follow-up angiography in $1 / 14$ rabbits of the MWCE coils and in 5/13 rabbits of the hybrid coil group. The mean serum levels for $\mathrm{Fe}$ were $31,39 \mathrm{ymol}(\mathrm{Zn} 49,18 \mathrm{ymol} / \mathrm{l})$ prior to implantation and $31,35 \mathrm{ymol} / 1(\mathrm{Zn} 46,20 \mathrm{ymol} / \mathrm{l})$ at the last follow-up in the hybrid coil group. In the MWCE group the mean serum levels for $\mathrm{Fe}$ were $31,12 \mathrm{ymol}\left(\mathrm{Z}_{\mathrm{n}} 46,17 \mathrm{ymol} / \mathrm{l}\right)$ prior to implantation and $25,94 \mathrm{ymol} / 1(\mathrm{Zn} 38,03 \mathrm{ymol} / \mathrm{l})$ at the last follow-up. No local toxicity was encountered on histopathologic cutting-grinding specimens of the coiled subclavian artery. No excessive inflammatory response to the coil was observed. Examination of the heart, lung, liver, spleen, ovaries and kidneys showed no macroscopic or histopathologic signs of systemic toxicity neither in the hybrid coil nor in the MWCE group.

Conclusion: Degradable $\mathrm{Fe} / \mathrm{Zn}$ hybrid coils show no local or systemic toxicity 3 to 12 months after implantation into the subclavian artery of New Zealand white rabbits. Implantation of $\mathrm{Fe} / \mathrm{Zn}$ coils does not result in elevated serum iron or zinc levels.

\section{P260}

MRI guided interventions: a new concept for automated tip detection of endovascular catheters

T. Kuehne, S. Weiss, M. Zenge, C. Lilje, J. Weil

University Hospital Hamburg, Pediatric Cardiology, Hamburg, D

Purpose: Fast and reliable definition of catheters and guide wires are crucial for accurate MRI guided cardiovascular interventions. So far, all MRI position monitoring techniques are confronted with (1) the challenge to generate sufficient contrast between the instrument and its surrounding anatomy or (2) a potential patient risk due to heating effects of radiofrequency conducting components. Purpose of this study was to construct and test an endovascular catheter for MRI guided intervention which (1) provides a fast and reliable identifiable MR signal, (2) is suited for projection based automated detection of the catheter tip and, (3) is bioelectrically safe.

Material and Methods: We introduce a concept based on a pair of resonance circuits $(\mathrm{RC})$ as fiducial markers of endovascular catheters. Each RC comprised a small copper coil and a microcapacitor. The coils were superimposed and positioned perpendicular to each other in order to decouple the RCs. Decoupling provides excitation of the fiducial marker at any catheter orientation. Two photodiodes were connected in parallel with the microcapacitors in order to optically detune the RCs, e.g. to switch the MR signal on and off. The photodiodes were connected over a fiber optic to a light source placed at the proximal end of the catheter. All catheters were tested in-vitro using a flow phantom simulating arterial and venous vasculature. MR imaging was performed on a 1.5 Tesla ACS-NT scanner (Philips, Best, NL) using an interactive real time imaging sequence.

Results: The fiducial marker provided a bright and sharp MR signal at any catheter orientation. The signal of the fiducial marker was fast and reliable identifiable against any background. Illumination of the photodiodes detuned the RCs, which in turn switched the MR signal off. By this means the tip of the catheter could be automatically localized using a projection based real-time tracking algorithm.

Conclusion: Endovascular catheters with integrated RCs are a promising tool for MRI guided cardiovascular interventions. The catheters are bioelectrically safe, provide excellent signal and can be automatically localized.

P261

The Buttonseal centering on demand device: combined American and European experience

R. Zamora, P.S. Rao, S. Sanhu, J. Chandar, M. Poursanov,

V. Varvarenko, E.B. Sideris

Division of Pediatric Cardiology, University of Arizona, Tucson, Arizona, USA

The Buttonseal centering on demand device (COD) has been implanted for secundum atrial septal defect (ASD) occlusion in European and American clinical trials, including an FDA supervised clinical trial, since 1998 . The COD is a polyurethane covered stainless steel device with low nickel content. It is a multipurpose occluding device used as centering in single defects and as non centering for fenestrated defects or for cases requiring septal rim augmentation. The COD can be also used in combination with an inverted counter-occluder (combination device) CODs were applied in 80 patients with single (73) or fenestrated (7) ASD secundum defects. The defect size varied from $6-30 \mathrm{~mm}$ and the device size from $25-60 \mathrm{~mm}$. Regular COD devices were applied in 60 cases and combination devices in 20 . Seventy five of the implantations resulted in effective occlusions (full occlusions and trivial shunts) and five in small to moderate residual shunts. The addition of the inverted counter-occluder improved the initial occlusion rate in $18 / 20$ cases used. Three cases with moderate residual shunts required re-interventions or surgery.

There were no serious complications on implantation or followup. There were no embolizations, unbuttonings or wire fractures. A thrombus was detected on an occluder by echo during implantation but disappeared with TPA treatment in a few hours.

In conclusion, the COD is a multipurpose device with high occlusion and low complication rates applicable to defects up to $30 \mathrm{~mm}$ with a good rim. The optional use of the inverted counteroccluder can increase the occlusion rates. 


\section{P262}

\section{Transcatheter patch occlusion of heart defects}

E.B. Sideris, S. Toumanides, B. Macuil, B. Alekyan, V. Varvarenko,

S. Moulopoulos

Athenian Institute of Pediatric Cardiology, Athens, GR

The effectiveness and safety of transcatheter patch (TP) heart defect occlusion was examined in the occlusion of various heart defects in 33 patients including: atrial septal defects (ASDs), perimembranous ventricular septal defects (VSDs) and large patent ductus arteriosus (PDA). Twenty five ASDs were repaired by TPs. Twenty three of them were inappropriate for disk device repair because of inadequate septal rim, or short septal length. The median age and defect size were 36 years and $26 \mathrm{~mm}$ respectively. All patients had effective occlusions, except for two with significant residual shunts; one of them received a second patch six months later with full occlusion. The occlusion status was maintained on follow-up (median 12 months) and the patched septum tended to become indistinguishable from the normal. Five VSIDs were repaired by TPs. All of them were inappropriate for disk device repair because of deficient subaortic rim. The median defect size was $5 \mathrm{~mm}$ and the median patient age 2 years. All VSDs had effective occlusions without complications. Three large PDAs with balloon diameters $12-22 \mathrm{~mm}$ were repaired by TPs after disk device failures. All PDAs had full occlusions; there were no complications. In conclusion, the TP appears effective and safe acutely and on short term follow-up in the occlusion of a variety of heart defects, mostly inappropriate for disk device repair. Despite the disadvantage of the 48 hour ambulation of the TP patient, large clinical trials comparing the TP to disk devices are justified.

\section{P263}

Percutaneous trans-aortic occlusion of patent ductus arteriosus using a new versatile angiographic and delivery catheter

A. Lorber, A.Z. Gazit, A. Khouri, Y. Schuratz, F. Freudental Department of Pediatric Cardiology, Rambam Medical Center, Haifa, IL

Objective: To evaluate the usefulness of Patent Ductus Arteriosus occlusion using a new catheter which combines good angiographic properties, easy manipulation across the duct, documentation of aortic and pulmonary artery pressure, and coil delivery for percutaneous trans-aortic ductal occlusion.

Design: Prospective study.

Setting: Single plain, $\mathrm{C}-\mathrm{arm}$, Siemens catheterization laboratory at the Rambam Medical Center, Technion Faculty of Medicine.

Patients: Thirty patients (Thirteen females, and seventeen males) who met the clinical and echocardiographic criteria for percutaneous closure of PDA were catheterized. The patients selected for this study had a small to moderate Patent Ductus Arteriosus with a conal or tubular shape, adequate aortic ampula and normal or mildly elevated pulmonary artery pressure. The median age was 4.8 years (Range: 10 months -20 years).

Intervention: Patients were catheterized by the trans-aortic approach using the new angiographic and coil delivery catheter, specially designed for this procedure. Patent ductus Arteriosus was closed in every case. A single pfm DuctOcclud coil was delivered in twenty eight patients. Two patients had pfm NitOcclud coils for ductal occlusion.

Measurements and main results: Screening time was measured and found to be $50 \%$ shorter then the measured screening time using other catheters in our laboratory. Only one catheter and one guide wire were used per procedure with no need for exchange wires or establishment of an arterio-venous loop. Procedure time was also reduced by $50 \%$. A minimal residual shunt demonstrated by color-flow Doppler mapping was present in two patients. Conclusions: The new combined angiographic and coil delivery catheter is most suitable for trans-aortic Patent Ductus Arteriosus coil occlusion, significantly shortening procedure and screening time.

\section{P264}

Transthoracic versus transesophageal echocardiografic findings in 233 consecutive patients before interventional ASD occlusion - a single center experience with four different devices

M. Vogt, J. Gerstl, M. Hauser, W. Sebening, Th. Genz, A. Eicken, J. Hess

German Heart Center Munich, Pediatric Cardiology, Munich, D

Between $7 / 96$ and $9 / 01,233$ consecutive patients were examined before interventional occlusion of ASD/PFO (simple ASD $n=155$, complex ASD $n=24$, PFO and stroke $n=54$ ).

The Aim of the study was to evaluate the sensititvity of TTE versus TEE comparing four echo items: size, position, number of ASD, size of rims. To compare echofindings in patients with and without device (native size, stretched ballon diameter). To define reasons for unsuccessful device implantation and to define specific advantages of different devices.

Results: TTE and TEE findings were equal in 70\% defining rim size, in $73 \%$ defining native defect size, in $76 \%$ defining position of the defect and in $83 \%$ defining number of ASDs. In $39 \%(\mathrm{n}=91)$ of the patients a device could not be implanted: in $46 \%$ defect size was too large, in $16 \%$ mutiple ASDs could not be occluded with one device and in 13\% rim size was too small. In 55 pts. the procedure was stopped after TEE, in 31 after ballon sizing and in 5 pts a device was removed in the same session. Occlusion rate rose from $38 \%$ with the AngelVings system, $30 \%$ with the CardioSeal Starflex to $73 \%$ with the use of Amplatzer devices. Native size and stretched diameter of ASD were significantly larger in patients with Amplatzer devices than in those with AngelWings, CardioSeal Starflex or Helex $(\mathrm{p}<0.05)$.

Conclusion: TEE is superior to TEE concerning native size, rim size, position and number of ASDs. Main reason for unsuccessful device implantation were large native size and multiple ASDs. The implementation of Amplatzer devices improved success rate from 30 to $73 \%$. As a consequence of this we use CardioSeal/Starflex in patients with multiple ASDs or PFO with septal aneuryms only

\section{P265}

What are the causes of non percutaneous closure of ASD with the Amplatzer Septal Occluder? Report of 121 consecutive patients

F. Godart, D. Satsou, A. Vincentelli*, C. Francart, G.M. Brevire, A. Prat*, C. Re'

Paediatric Cardiology, Cardiac Hospital, Lille, F

The purpose of this study is to evaluate the possible causes of non transcatheter closure of ASD with the Amplatzer Septal Occluder (ASO) over a period of 2 years.

From January 1999 to December 2000, 121 consecutive patients were referred for closure of ASD with significant left-to-right shunt. An attempt of transcatheter closure with the ASO was realised in 68 patients (Group I); sixty patients were surgically repaired (Group II)

The sex ratio was $2.5 \mathrm{female} / \mathrm{male}$. Age in Group I was $30 \pm 19$ years and in Group II $16 \pm 29$ years. ASD diameter (echo 
and/or stretched diameter) was $21.8 \pm 5.6 \mathrm{~mm}$ in Group I and $26.8 \pm 4.2 \mathrm{~mm}$ in Group II. ASD dimension during surgery was $33.0 \pm 8.1 \mathrm{~mm}$. ASO implantation succeeded in 61 patients $(90 \%)$. Causes of non-transcatheter closure were: insufficient rim $(\mathrm{n}=27)$ (superior, $\mathrm{n}=11$; inferior, $\mathrm{n}=7$; retro-aortic, $\mathrm{n}=6$; coronary sinus, $n=3$ ), associated partial anomalous venous drainage $(n=15)$, large defect related to the atrial length $(n=11)$, and multiple defects $(n=7)$. Patients of Group II had tendency to be younger (age $<18.3$ years), to have lower weight $(<40 \mathrm{~kg})$, higher QP/QS $(>2.03)$ and larger defect (stretched diameter $>25 \mathrm{~mm}$ ) but on multivariate analysis, no criteria reached significant statistical level.

Transcatheter closure of ASD feasible in $57 \%$ of patients referred for ASD closure without anomalous partial venous drainage. The main reason for failure is the absence of rim which is usually well evaluated by echocardiography. Absence of retro-aortic rim is no longer a contra-indication.

\section{P266}

Assessment of atrial septal defect size with 3D-echocardiography: comparison with balloon method. T. Abdel-Massih, Y. Aggoun, Y. Boudjemline, G. Agnoletti, M.Y. Dousteblazy, P. Bonhoeffer, D. Bonnet, D. Sidi, P. Acar Service de cardiologie pediatrique - Necker enfants malades, Paris, $F$

Transcatheter closure of atrial septal defect (ASD) is an alternative approach to surgery in selected patients. Balloon stretched diameter (BSD) is considered as the standard way of measuring ASD size. Three-dimensional transesophageal echocardiography (3DTEE) provides views of the ASD allowing its measurement and identifying its spatial relation with neighboring structures. In this study we aimed to compare the balloon and 3D-TEE methods to measure the ASD size before transcatheter closure. Seventy-six consecutive patients were enrolled for ASD device closure using the Amplatzer septal occluder. 3D-TEE and balloon sizing were adequately performed in 70 patients before the defect closure. The mean maximal diameter measured by 3D-TEE was $20 \star 15 \mathrm{~mm}$ (range 10 to 28 ) while the mean BSD was $22 \star 5 \mathrm{~mm}$ (range 9 to 31 ). When comparing the $3 \mathrm{D}-\mathrm{TEE}$ and transcatheter measurements, there was a good correlation between the two methods $(y=3.15+0.77 x ; r=0.8)$. The defect as viewed by $3 D-T E E$ was unique in 54 patients and multiple in 16 patients. In patients with a unique defect, the correlation between the two methods was high $(y=1.74+0.84 x ; r=0.85)$ while patients with multiple ASDs, the correlation was poor $(y=12.4+0.4 \mathrm{x} ; \mathrm{r}=0.45)$. 3D-TEE and transcatheter methods are two complementary techniques for the success of transcatheter ASDs closure.

\section{P267}

Covered stents in the treatment of early postoperative pulmonary artery obstruction after Fontan

O. Stumper, B. Nagel, T. Robertson, D.J. Barron, W.J. Brawn

Children's Hospital, Birmingham, UK

Pulmonary artery obstruction early after a Fontan procedure imposes significant perioperative morbidity and mortality. We postulated that such lesions may be amenable to early postoperative balloon angioplasty or transcatheter stent implantation.

Over a two year period 68 children underwent completion of an extracardiac Fontan procedure without early mortality. Two children had significant early postoperative problems attributable to raised right heart pressures and pulmonary artery obstruction.

Patient $\mathrm{Nr} 1$ was documented on catheter 17 days post procedure to have complete occlusion of the left PA. PV wedge angiograms showed patent distal vessels. The occluded area was perforated using a guidewire. This was followed by balloon angioplasty and transcatheter covered stent implantation. She made good recovery and was discharged on day 5 post procedure.

Patient Nr2 expressed signs of an excessive R-L shunt and pulmonary artery obstruction on Day 2 post extracardiac fenestrated Fontan procedure. Cardiac catheter documented distortion of the left pulmonary artery despite extensive surgical reconstruction. Transcatheter implantation of a covered stent established a very satisfactory morphology and flow to the left lung artery. The fenestration was reduced in size surgically. The patient made very rapid haemodynamic recovery following this

Conclusion: Early postoperative pulmonary artery obstruction or distortion after a Fontan procedure can be treated safely and effectively by transcatheter covered stent implantation. This technique should be preferred above surgical re-intervention.

\section{P268}

Covered stents fare better in maintaining patency of ductus arteriosus: preliminary results in newborn lambs S. Radio, S.F. Froemming, C. Shaffer UN/CU, Children's Hospital, Omaha, USA

Objective: Most patients with cyanotic congenital heart disease require systemic to pulmonary artery shunt (SPS) after birth. This palliation can be avoided if there is a device available to maintain patency of the ductus arteriosus (DA). The purpose of this study was to test short term patency of a new Amplatzer device (AMP) and a new covered stent in DA of lambs.

Methods: The AMP (1 cm long, $4 \mathrm{~mm}$ diameter) was made of Nitinol wires. The JOSTENT (16 $\mathrm{mm}$ length, $4 \mathrm{~mm}$ diameter) was made of stainless steel and covered with PTFE graft. All lambs were placed on Prostaglandin E1 before cardiac catheterization (CATH). Nine newborn lambs underwent CATH for placement of device. In 7 lambs, the DA was crossed with the help of a wire. The AMP was deployed using a $5 \mathrm{~F}$ sheath in 4 lambs. The JOSTENT was deployed in 3 lambs. Cineangiograms were obtained after placement of the devices and before euthanasia. The lambs were followed for two months.

Results: In all lambs, angiograms performed after placement of the device revealed good flow to the pulmonary arteries. In the lambs with AMP, cineangiograms after 2 months revealed near occlusion of the DA in 1 and $2 \mathrm{~mm}$ opening in the remaining 3. Gross examination revealed ingrowth of ductal tissue through the wire mesh and from the edge of the device. In the lambs with JOSTENT, the lumen was wide open after 2 months. There was no evidence of tissue ingrowth through the stent, but there was some ingrowth of tissue from the edge of the stent in 1 lamb.

Conclusions: Both devices maintained DA patency acutely. However, covered stents appear to maintain patency for longer intervals by preventing ingrowth of ductal tissue. Covered stents may be useful in selected patients to maintain patency of DA. The advantages include avoidance of thoractomy or sternotomy, possible cardiopulmonary bypass, and complications associated with SPS.

\section{P269}

A new era in the treatment of HLHS: transcatheter Stage I palliation and Stage III completion J.P. Cheatham, M. Galantowicz, K. Amplatz, A. Tower, C.S. Kleinman, H. Munro, K. Spalding, S. Hill, D. Hsu Nemours Cardiac Center, Orlando, Florida, USA

Hypoplastic Left Heart Syndrome (HLHS) is repaired by 3 staged open heart surgical procedures. In order to potentially improve 
survival \& outcome, as well as decreased exposure to blood in Jehovah Witness (JHW) pts, we offered transcatheter palliative treatment for Stage I \& completion for Stage III repair using custom made devices \& stents after appropriate IRB notification, consents, and manufacturer support were obtained.

Methods: From August through December 2001, we treated 5 newborns ( $3 \mathrm{JHW}$ ) for Stage I using either: 1) PDA stent (PDAS) followed by closed surgical RPA/LPA banding $(n=3)$; or 2 ) PDAS \& implantation of Amplatzer PA Band Devices (APABD) $(\mathrm{n}=2)$. Sixteen $\mathrm{mm}$ long IntraStents (TM) were expanded to 8 or $9 \mathrm{~mm}$ to maintain the PDA \& $6 \mathrm{~mm}$ APABD with two $2 \mathrm{~mm}$ fenestrations were implanted in the RPA/LPA. We also treated 2 infants $(1 \mathrm{JHW})$ for Stage III using covered NuMED CP stents (CCPS) to create a "lateral tunnel". An 8-zig $50 \mathrm{~mm} \& 8$-zig $55 \mathrm{~mm}$ CCPS were initially implanted \& expanded to $16 \mathrm{~mm} \mathrm{\&}$ $14 \mathrm{~mm}$ respectively. A shorter $34 \mathrm{~mm} \& 22 \mathrm{~mm}$ CCPS delivered coaxially were required for eventual success in both prs.

Results: PDAS were successfully implanted in all 5 newborns. Delivery of APABD was successful in both LPAs, but only 1 RPA. Transient decreased BP, decreased HR, or heart block occurred \&. required circulatory support. CCPS were successfully implanted in the 2 infants, completing the "lateral tunnel" with increased $\mathrm{O}_{2}$ sats $90-92 \%$.

Follow-up: Two newborns required later balloon atrial septostomy, 1 required balloon aortic angioplasty for re-CoA. Two newborns died: 1 from sepsis \& 1 at home with genetic \& other medical problems. One infant required re-expansion of CCPS to include the medial hepatic vein.

Summary: 1) Stage I palliation of HLHS using transcatheter techniques \& custom devices, with or without surgery, is possible. 2) Placement of APABD is technically challenging, causing hemodynamic instability. Better materials \& delivery systems are needed. 3) Completion of Stage III using CCPS is possible \& offers an alternative treatment in selected patients.

\section{P270}

Transcatheter arterial embolization of large and medium size pulmonary arteriovenous fistula using the Amplatzer Occluding devices: immediate and medium term results F.F. Pinto, L. de Sousa, C. Trigo, A. Agapito, A.Mata Antunes e Sashicanta Kaku

Hospital de Santa Marta - Servio de Cardiologia Peditrica, Lisboa, $P$

Purpose: The aim of this study was to assess the effectiveness of percutaneous arterial embolization for multiple PAVF with the Amplatzer Occluding devices; through the results of immediate and medium term follow-up.

Introduction: Pulmonary arteriovenous fistula (PAVF) of large and medium size may cause a number complications if left untreated. Transcatheter embolization of the feeding arteries is a relatively new approach and information concerning its efficacy and medium or long term results are scarce, particularly in patients with multiple PAVF. Transcatheter embolization of PAVF has been performed in our Center since 1987 using several occluding available devices: detetchable balloons; spring coils and most recently Amplatzer Occluding Devices (AOD).

Patients and methods: Transcatheter embolization of 15 medium and large size PAVF using AOD was performed in seven patients (three man and four women), between 1999 and 2001 who were followed-up for at least one year. All patients had multiple PAVF and three of them had already been previously treated with other embolization devices (two detachable balloons and 11 coils) which recanalized. The size of the 15 PAVF occluded ranged from 4 to $15 \mathrm{~mm}$ (mean $7.2 \mathrm{~mm}$ ); one patient had a huge fistula $(15-\mathrm{mm})$.
We used 11 PDA occluding devices and one septal occluding device. And one embolization was performed twice in five patients and once in two patients. All patients were recatheterized one year after the embolization.

Results: No severe complications were seen in the short-term follow-up. Arterial oxygen pressure improved in all patients without oxygen supplementation. All patients are well and alive after a follow-up ranging from one year to three years. All patients had immediate and complete occlusion of the shunt after embolization of the afferent arteries. Angiography at one year of follow-up disclosed complete occlusion of all treated PAVF and two new PAVF in two patients, which were occluded.

Conclusions: Transcatheter embolization of the feeding arteries of medium and large size PAVF with Amplatzer Occluding Devices is an efficacious and safe treatment, with excellent short and medium term results, specially in patients with multiple PAVF who are prone to acquire new fistula.

\section{P271}

Catheter closure of patent foramen ovale with the Amplatzer PFO occluder in 103 consecutive patients U. Krumsdorf, T. Trepels, K. Horvath, S. Ostermayer, K. Billinger, E. Zadan, H. Sievert

Cardiovascular Center Bethanien, Frankfurt, D

Background: Catheter closure of patent foramen ovale (PFO) has been proposed to prevent recurrent paradoxical embolism. We used the new Amplatzer PFO occluder in this indication.

Methods: Transcatheter PFO closure was attempted with the Amplatzer PFO occluder in 103 patients. The age ranged from 22 to 76 years (mean 48 12). 140 embolic events occurred in these patients prior to PFO closure. The diameter of the PFO ranged between 3 to $16.9 \mathrm{~mm}$ (mean 9 3) measured with balloon sizing. Results: The implantation of the device was successful in all patients. The device diameter was $25 \mathrm{~mm}$ in 96 and $35 \mathrm{~mm}$ in 7 patients. The procedure could be performed without general anesthesia in 100/103 patients. Mean fluoroscopy time was $5.74 .1 \mathrm{~min}$., including balloon sizing of the defect. Patients were discharged 0.8 0.2 days after treatment. Transoesophageal echocardiogram (TEE) showed a small residual shunt in 24 patients which persisted in 2 . A device perforation occurred in one patient after 24 hours (surgery uneventful). During follow-up of 0.03 to 26 months one recurrent embolic event occurred. 3 patients experienced paroxysmal atrial fibrillations (cardioversion and medical treatment successful in all of them). No thrombus formation could be detected.

Conclusion: PFO closure with the Amplatzer PFO occluder is a very safe procedure and prevents further embolic events. It can be performed as an outpatient procedure under local anesthesia. Acute and midterm complications are rare.

\section{P272}

Transcatheter closure of ventricular septal defects

J.V. De Giovanni, S. Thorne, M. Chaudhari, J. Vettukattil Birmingham Children's Hospital, Birmingham, UK

Between 1994 and 2001, 9 patients with a ventricular septal defect (VSD) were considered for catheter closure. Indications included haemodynamic reasons, haemolysis or potential for bacterial endocarditis.

Nine patients were considered, but the procedure was abandoned in 1 due to cardiovascular instability. The procedure was successful in 7 out of the 8 performed, with failure to satisfactorily deploy the device in 1 patient and this was retreived. 
The age ranged from 3 weeks to 62 years. A total of 11 devices were used in 8 patients, 1 in each of 6 patients, 2 in one and 3 in another. The devices used included 4 Rashkind double umbrella devices, 1 coil, 1 Amplatzer ASO and 5 Amplatzer VSD devices. Of the 8 patients in whom deployment was performed, 5 were native congenital, 1 was residual post-surgical, 1 post-infarct and post-surgical, and 1 post-traumatic and post-surgical. All VSDs were ballooned-sized to determined device size.

Complications: Transient bradycardia and hypotension were common. External cardiac massage was required in 2 , leading to abandonment of the procedure in 1 case; the latter had surgical repair and was found to have tears in the mitral and tricuspid valves, both of which were repaired. There were no other complications and all the successful ones showed clinical benefit.

Conclusion: Transcatheter closure of VSDs is becoming increasingly used particularly with specifically designed devices. There is a role for this in isolated VSDs, as part of a combined strategy with surgery or in residual post-operative defects. The procedure is, however, more complex than closure of atrial setal defects.

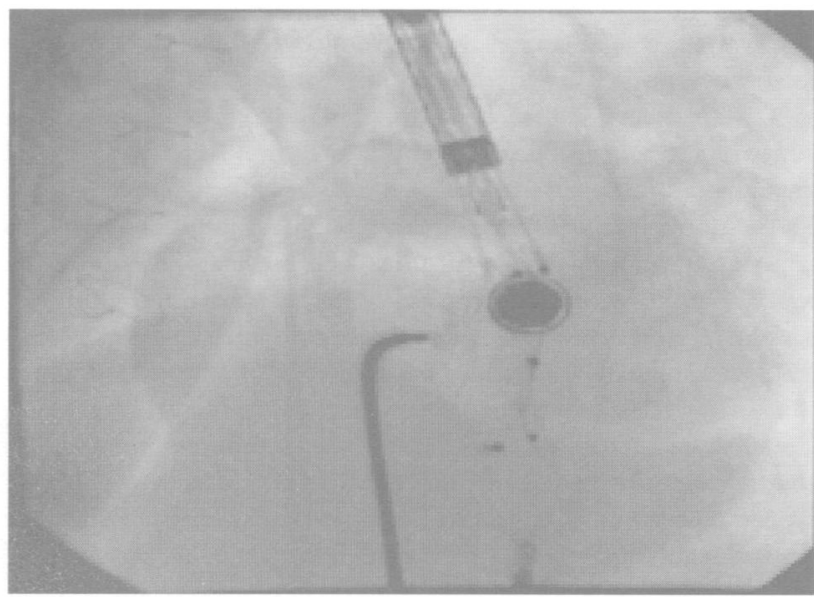

P273

What are the different occluders available for transcatheter closure of coronary artery fistulas? Experience with 3 different devices C. Rey, F. Godart, G.M. Brevire, C. Francart

Paediatric Cardiology, Lille, $F$

The purpose of this work is to report transcatheter closure of coronary artery fistulas with 3 different devices: detachable balloon, detachable coil, and Amplatzer Duct Occluder (ADO).

Nine cardiac catheterizations were performed in 7 patients (age 8 months to 40 years, mean 13.9 years, median 7 years). The coronary fistula involved the right coronary artery in 5 patients (drainage to the right atrium, $n=4$, and drainage to the right ventricle, $n=1$ ) and the left coronary artery (1 drainage to the right ventricle and 1 in the coronary sinus). All patients had a continuous murmur and the infant was under digitalis and diuretics. One child had undergone 2 years before unsuccessful surgical ligation of the fistula with a persistent shunt. One patient had transcatheter closure of both the coronary fistula and a patent ductus arteriosus. Transcatheter closure was realised under local anaesthesia except in the 3 youngest. Closure was realised with a silicone detachable balloon $(n=6)$, detachable coil $(n=2)$, and ADO $(n=1)$. One embolization of detachable balloon in pulmonary artery occurred after successful occlusion leading to subsequent implantation of $A D O$.
To conclude, occlusion of large fistulas was effective in 6 of 7 patients using 3 different devices. Devices were released after ECG control and angiography to confirm the correct placement. Choice of the devices is mainly related to the anatomy of the fistula.

\section{$\mathrm{P} 274$}

Use of a single Amplatzer device to close multiple ostium secundum atrial septal defects

J.B. Thambo, R. Ibrahim, P. De Guise, E. Piette, M.J. Raboisson, M. Brassard, J. Mir

Sainte Justine Hospital, Montral, Quebec, CAN; Bordeaux, F

Objective: To explore whether the closure of multiple ASDs with a single Amplatzer device is feasible, effective and safe.

Population and Method: We compared retrospectively the 25 patients in whom percutaneous closure of multiple ASDs was attempted between 12/1997 and 02/2001 (group I) with a control group of 25 patients with a single ASD (group II). Mean age $(9,4$ vs $5,8 \mathrm{yrs})$, weight $(31,5 \mathrm{vs} 19,4 \mathrm{~kg})$ and height $(123,6 \mathrm{vs} 108,6 \mathrm{~cm})$ were higher in group I, while the $\mathrm{Qp} / \mathrm{Qs}$ was comparable $(1,9 \pm 0,6$ vs $2,0 \pm 0,9)$. In group $I$, the mean number of defects was 2,6 (range: 2 to 4 ). The biggest defect at TEE measured in average $10,0 \mathrm{~mm}(2,5$ to 20$)$ and the smallest $3,7 \mathrm{~mm}$ (1 to 10). In group II, the defect measured $12,1 \mathrm{~mm}$ ( 6 to 28 ). In Group I, the device was deployed in the largest defect, clearly oversizing it (mean device size $18,9 \mathrm{~mm}$, range 12 to 30 ), covering the additional defects with the discs. In group II, the device was selected according to the stretched diameter of the defect (mean 16,9 mm, range 6 to 32 ).

Results: A single device was successfully deployed in $96 \%$ vs $100 \%$ patients respectively. Mean fluoroscopy time (23,8 vs. $20,2 \mathrm{~min}$ ) and procedure time $(78,2$ vs. $60,4 \mathrm{~min})$ were comparable. Immediate residual shunt was documented by TEE in $52 \%$ vs. $84 \%$ of cases respectively $(\mathrm{p}<0.05)$. At one year, however, a shunt was documented in only one patient in group I. Right ventricular dimension regressed in both groups, but more and faster in group II. The only complication was an intermittent 2 nd degree $\mathrm{A}-\mathrm{V}$ block in one patient in group $\mathrm{I}$, which resolved spontaneously.

Conclusion: Closure of multiple ASDs using a single Amplatzer device is safe and effective, yielding results comparable to single ASD. While a residual shunt is frequent immediately, it tends to become undetectable with time, as the device gets endothelialized, thus covering the additional defects. Multiple ASDs can be closed routinely percutaneously and we emphasise the usefulness of TEE to guide the closure.

\section{P275}

Long term result of balloon dilation of pulmonary valve stenosis in $\mathbf{1 0 0}$ neonates and children

R.S. Bader, S. Al Youssef

Hospital for Sick Children, Toronto, CDN

To evaluate the long term (up to 15 years) outcome for patients who had balloon pulmonary valvoplasty (BPV).

Patient records, catheterisation data, cineangiograms and echocardiograms of 100 consecutive children (mean age 4.14 years, range 1 day to 17 years) undergoing attempted BPV between August 1984 and December 1987 were reviewed. The follow up period varied between 7.47 years and 15 years, a mean of $13.5 \pm 2.56$. BPV was accomplished in $86(86 \%)$ of 100 attempts. Immediately after dilation, the transvalvular peak to peak systolic gradient 
decreased from $100.29 \pm 48.25 \mathrm{mmHg}$ (mean SD, range 30 to 235) to $19.51 \pm 8.96 \mathrm{mmHg}$ (range 10 to 47$)$, (p 0.0001) and right ventricular/femoral systolic pressure ratio decreased from $1.25 \pm 0.43$ (range 0.5 to 2.6 ) to $0.66 \pm 0.22$ (range $0.2-1$ ) (p 0.0001 ). Oxygen saturation measured by percutaneous oximetry increased from $80 \pm 7 \%$ to $92 \pm 4 \%$ (p 0.0001$)$. There were 14 primary failures $(14 \%)$ with residual valvular gradients $>36 \mathrm{mmHg} .13$ patients $(13 \%)$ had repeat balloon dilation.

At early follow up (1 year) after initial BPV, in 98 patients (98\%) mean gradient was $12.6 \mathrm{mmHg} \pm 16(\mathrm{p} 0.0001)$. At medium term follow-up (5 years) 75 patients $(75 \%)$ had a mean gradient of $14 \mathrm{mmHg} \pm 16 \mathrm{mmHg}$, at long term follow up (10 years) 62 patients $(62 \%)$ had a mean gradient of $15 \mathrm{mmHg} \pm 6 \mathrm{mmHg}$, at 15 years follow up 58 patients $(58 \%)$ had a mean gradient of $12 \mathrm{mmHg} \pm 2 \mathrm{mmHg}$. Five patients had successful surgical intervention due to dysplastic or non-valvular pulmonary stenosis. The success rate of BPV being $86 \%, 78 \%, 62 \%$ and $58 \%$ at $1,5,10$ and 15 years follow up.

BPV affords both acute and long term sustained haemodynamic relief of valvular obstruction in patients with moderate to severe pulmonary stenosis and offers an excellent alternative to open or closed heart surgery

\section{P276}

Transcatheter aortic valvuloplasty assisted by right ventricular pacing

F. Ing, N. Boramanand, J. Matheurson, K. Maginot, J. Perry

Children's Hospital of San Diego, San Diego, USA

Background: Transcatheter aortic valvuloplasty (TAV) is a wellestablished treatment modality for congenital aortic valve stenosis. However during dilation, inadvertent balloon movement (due to the contractile force of the left ventricle) can increase risk of unnecessary trauma to the aortic valve apparatus resulting in aortic insufficiency (AI). A new technique was developed to stabilize balloon position during dilation.

Methods: Standard transfemoral retrograde techniques were used for TAV. A pacing catheter was inserted into the right ventricle (RV) apex and just prior to balloon inflation, the heart was paced at a faster rate in order to decrease the stroke volume and left ventricle (LV) systolic pressure. The lower LV stroke volume and systolic pressure result in a more stable balloon position. Balloon inflation was carried out in the usual manner and following balloon deflation, RV pacing was discontinued. Echocardiographic and cath lab data were reviewed in all patients who underwent TAV assisted by RV pacing between 9/99 and 12/01.

Results: Fourteen patients underwent TAV assisted by RV pacing (32 inflations). Mean age and weight were 10.012 .3 years and $35.531 .1 \mathrm{~kg}$ respectively. The aortic valve gradient decreased $71 \%$ from 70.220 .5 to $20.09 .0 \mathrm{mmHg}$. The average balloon to annulus ratio was 0.940 .07 . RV pacing increased the heart rate by an average of $8029 \%$ and decreased LV systolic pressure by $3612 \%$. Balloon position remained stable during inflation in all except for one when there was loss of capture resulting in a premature ventricular contraction (PVC). Review of the fluoroscopic and hemodynamic recording of that inflation indicated the balloon "milked" forward during the PVC. Normal sinus rhythm returned in all cases immediately after RV pacing was discontinued. No change in AI or trace AI was seen in 11 pts. The development of mild AI was seen in $3(1+$ in 1 and $2+$ in 2 pts).

Conclusion: RV pacing during TAV is safe and stabilizes balloon position during aortic valve dilation. Larger series and longer follow-up are warranted to confirm that stable balloon position during TAV decreases the incidence of aortic insufficiency.

\section{P277}

Long term follow-up of transvenous cardiac pacing in children. Nine-year experience: 155 patients, 232 interventions and 1587 outpatient visits

A. Celiker, O. Baspinar, S. Ozer

Department of Pediatric Cardiology, Hacettepe Medical School, Ankara, TR

Background: Permanent transvenous cardiac pacing has become a frequently used therapeutic modality. The aims of this study were to asses morbidity, mortality and long-term prognosis of children. Methods: A hundred fifty five patients (male $40 \%$, female $60 \%$ ), mean age, 9.14 .7 years, ( 9 months to 22 years), mean body weight, $28.814 .9 \mathrm{~kg}$, $(6.4$ to $80 \mathrm{~kg})$ who received permanent endocardial pacemakers from January, 1993, to December, 2001 were reveiwed retrospectively using patient records. Ventricular pacing was used in 123 patients, $70.6 \%$ of cases $(64.3 \%$ with rate response capabilities), single lead VDD pacing was the pacing mode in 22 patients, $12.6 \%$ of the cases. Atrial pacing was 13 patients, $7.4 \%$ and dual chamber devices were implanted to $16,9.1 \%$ of patients. The main indication for pacing was atrioventricular block (AVB) followed by sinus node dysfunction (SND). The incidence of AVB, postoperation was $49.6 \%$, congenital was $24.5 \%$, acquired and post $A V$ node ablation was in $4.5 \%$ patients, combination of AVB and SND was in $3.2 \%$ patients. The incidence of SND was $21.9 \%$. Subclavian puncture $(96,1 \%)$ and cephalic cutdown $(3,8 \%)$ were used for lead implantation in our patients. Electrodes implanted were of 3 types: $55.1 \%$ steroid eluting active, $35.8 \%$ steroid eluting passive, and $9.1 \%$ non-steroid eluting passive fixation. The mean follow-up period was 37.728 .9 months (range 1 to 120). During follow-up, 77 patients required revision and 46 patients had 22 minor and 43 major complications. There were a total of 6 deaths, 3 of which were direct related to the patients underlying cardiac disease.

Conclusions: Transvenous permanent pacemaker insertion in children can be used safely and efficaciously, as with all invasive procedures, complications are possible and sometimes lead or generator revision may be necessary.

\section{P278}

Cardiac catheterization is feasible and safe in patients on extracorporeal life support after cardiac surgery for congenital heart disease

C. Fink, M. Peuster, G. Hausdorf

Pedatric Cardiology, University of Goettingen, Goettingen, $D$

Objective: To report our experience with cardiac catheterization in patients on extracorporeal life support (ECMO) after cardiac surgery.

Methods: Review of all cardiac catheterizations in patients on ECMO after cardiac surgery.

Patients: Mean age at catheterization was 145 days (range 4332 days) Diagnoses: d-TGA $(n=4)$, Bland-White Garland (BWG)Syndrome $(n=1)$, Tetralogy of Fallot $(n=1)$, double-outlet right ventricle with hypoplastic left ventricle $(n=1)$, total anomalous pulmonary venous drainage (TAPVD) $(\mathrm{n}=1)$.

Cardiac Surgery: Arterial switch operation $(n=4)$, transanular patch reconstruction of the right ventricular outflow tract $(n=1)$, Takeuchi repair of BWG-syndrome $(n=1)$, Glenn operation $(\mathbf{n}=1)$. ECMO was continued postoperatively since weaning was not possible in the operating room.

Results: In the patient after patch reconstruction of the pulmonary bifurcation a severe obstruction of the left pulmonary artery was identified 5 days postoperatively and successfully treated with stent implantation, the patient was weaned from ECMO 2 days postinterventionally. In the patient after Takeuchi repair of $\mathrm{BWG}$ syndrome, a stenotic left coronary artery could be identified and 
the patient was re-operated successfully, ECMO was stopped 7 days after reoperation. In the 4 patients after arterial switch operation, a stenotic coronary artery could be excluded in 3 patients. Weaning from ECMO was possible 6 and 7 days postoperatively when cardiac recompensation occurred, one patient died from cardiac failure 4 days postoperatively. 1 patient showed an obstruction of the left coronary artery and was reoperated successfully, however, severe RSV infection complicated the postoperative course and the patient died from respiratory failure. In the patient with correction of TAPVD cardiac catheterization could exclude an obstruction of the anastomosis but revealed severe pulmonary hypertension which was treated successfully by inhalation of nitric oxide and weaning from the ECMO was possible 3 days post catheterization. In the patient after Glenn operation, numerous venous collaterals were identified postoperatively and treated successfully by coilocclusion, ECMO could be stopped 6 days postinterventionally. Conclusion: In patients on extracorporeal life support after surgery for congenital heart disease, cardiac catheterization can be safely performed. It may improve outcome by timely diagnosis of the underlying pathology. Interventional therapy can be successfully performed in selected patients on ECMO.

\section{P279}

\section{Transcatheter occlusion of multiple atrial septal defects using the Amplatzer Occluder W.A.K. Radtke, V. Bandisode, M. Dilawar, W. Gottliebson, G. Shirali Medical Lniversity of South Carolina, Charleston, USA}

In order to determine efficacy and safety of transcatheter occlusion of multiple atrial septal defects (ASD), we report the single center experience with device occlusion of multiple ASD using the Amplatzer occluder. Twenty-seven patients were considered and underwent transesophageal echocardiography. Device implantation was attempted in 23 patients aged 2-50 years (median 10.3 years), weighing 10.3-100.5 kg (median $33.0 \mathrm{~kg}$ ) and accomplished in all. $\mathrm{Qp} / \mathrm{Qs}$ was $0.4-6.0$ (mean 2.1). Echo diameter of the individual defects ranged from 2 to $21 \mathrm{~mm}$. Four patients had three defects. Stretched diameters of the dominant defects were $6.5-31 \mathrm{~mm}$ (median $17.5 \mathrm{~mm}$ ). Device size ranged from $5 \mathrm{~mm}$ to $32 \mathrm{~mm}$ and two devices were placed in eight patients. Devices were placed in the dominant or central defect if a single occluder was likely to cover all defects. In marginal cases, a guide wire was kept in the second defect. Otherwise, two devices were placed in sequence. Selective cannulation of specific defects occasionally was difficult. Procedure time was 90-199 min (median $122 \mathrm{~min}$ ) with 12.5-37.5 min (median $20.6 \mathrm{~min}$ ) of fluoro time. There were no device embolizations or other major complications. One patient developed transient AV block that resolved spontaneously and atrial flutter was induced in one patient that converted spontaneously. Complete occlusion of all defects was achieved in 14/23 (61\%) at $24 \mathrm{hrs}$, in $16 / 22(73 \%)$ at 6 months and in $15 / 18(83 \%)$ after 1 year. All residual shunts were $3 \mathrm{~mm}$ or less in diameter. There were no late complications. We conclude that multiple ASD can safely and effectively be closed using the Amplatzer Occluder. Device size selection and placement depends on individual septal anatomy.

\section{P280}

Is echocardiographically guided closure of atrial septal defects suited for routine use?

I. Daehnert, A. Moeckel, P. Kinzel, P. Schneider, H.J. Haeusler

Klinik fuer Kinderkardiologie, Herzzentrum Leipzig, Leipzig, $D$

Aim: A method of echo-guided closure of atrial septal defects (ASD) using the Amplatzer septal occluder (ASO) without the use of fluoroscopy was described by Ewert et al. in
1999. However, it is still not known whether it is suited for routine use.

Methods: All children with simple ASD intended to have interventional closure after April 1st, 2000 were enrolled in a prospective protocol. Catheterization included standard pressure registrations and oxymetric shunt calculation. Correct catheter positions were confirmed by transthoracic echo. Balloon sizing and ASO implantation were performed under transesophageal echo-guidance.

Results: 53 patients (pts.), age 10 months to 20 (median 8.2) years, body weight 7.2 to 84 (median 26) $\mathrm{kg}$, have been enrolled so far ASD was excluded in one and judged too large for intervention after balloon sizing in five pts. ASD closure was attempted in $47 \mathrm{pts}$. and completed without fuoroscopy in 34 pts. In 13 pts. fluoroscopy had to be used because of problems with configuration or positioning of the device, successful ASD closure was possible in 10 of them In the remaining three pts. the device was withdrawn because no complete occlusion resp. no stable position was obtained.

Discussion: Fluoroscopy is not necessary for hemodynamic assessment and balloon sizing of ASD in children. The total occlusion rate in this series was $83 \%$ of intended and $94 \%$ of attempted implantation procedures. Fluoroscopy could be avoided in $75 \%$ of the pts. However, echocardiographically guided closure was feasible in only $77 \%$ of the successfully managable defects.

Conclusion: Radiation exposure of staff and pts. can be diminished by echo guided ASD-closure, which will be the method of choice in this institution. However, we will not hesitate to use fluoroscopy whenever there are problems.

\section{P281}

Migraine following device closure of atrial septal defect C. Duke, S.A. Qureshi, E. Rosenthal

Guy's Hospital, London, UK

Background: Transcatheter device closure of right-to-left shunting ASD/PFOs has been shown to reduce migraine severity. However, new onset migraine has been observed after ASD device implantation. We investigated how transcatheter ASD closure affected the frequency and severity of migraine in a consecutive series of patients

Methods: A structured questionnaire was used to conduct a telephone interview of patients who underwent ASD/PFO device closure between 1995 and 2001.

Results: 96 patients underwent device closure; 83 could be contacted and interviewed. $15 / 83$ patients $(18 \%)$ had a history of migraine before the procedure. Of these, $3 / 15(20 \%)$ had improved symptoms, $3 / 15(20 \%)$ had unchanged symptoms and $9 / 15(60 \%)$ had more frequent and severe migraines following device implantation. 6 previously unaffected patients $(7 \%)$ developed migraine. The 15 patients with exacerbated or new symptoms were all adults; indications for closure were left-to-right shunt in 8, TIA in 1, TIA and left-to-right shunt in 3 , stroke in 2 and decompression sickness in 1; there was an aura without headache in 3 and aura with headache in 12. Exacerbated or new migraine occurred in $11 / 41(27 \%)$ Amplatzer devices, 3/36 (8\%) CardioSEAL devices and $1 / 4(25 \%)$ Helex devices. In most affected patients, symptoms began either immediately or within days of the procedure and were initially daily or several times per week. $5 / 15$ patients described severe migraine that mostly resolved after 1 month and 10/15 described a protracted course with a gradual reduction in symptoms over many months, with most patients having some symptoms at latest follow-up (longest 2 years).

Conclusions: Migraine occurs relatively frequently in patients with unoperated ASDs (18\%). Exacerbated or newly precipitated migraine occurred in $18 \%$ of patients after device implantation, with initially intense symptoms improving gradually over many 
months in the majority. The effect is confined to the adult population and is seen with different device designs. It may be secondary to platelet activation on the left atrial disc of the device, with serotonin release affecting the cerebral vasculature. Those with a history of migraine may have cerebral vessels that react more markedly to the putative precipitating factor.

\section{P282}

\section{Adequate left ventricular preparation allows for} arterial switch despite late referral

A.F. Corno, M. Hurni, M. Payot, N. Sekarkki, P. Tozzi,

L.K. von Segesser

Centre Hospitalier Universitaire Vaudois, Lausanne, $\mathrm{CH}$

Objective. To evaluate the feasibility of arterial switch for TGA after late referral.

Methods. From March 2000 to August 2001 six children underwent arterial switch for TGA after LV preparation because of late referral. Mean age at referral was 8.3 months ( $3-25$ months), mean body weight $5.3 \mathrm{~kg}(3.7-9.3 \mathrm{~kg})$, mean oxygen saturation $57 \%$ $(50-72 \%)$.

Associated defects: restrictive VSD (3), aortic coarctation (1), partial anomalous pulmonary venous connection (1). Mean interval between referral and arterial switch was 3.7 months (1-7 months), with a mean of 1.5 (up to 3 ) surgical procedures for left ventricular preparation, including combinations of ASD creation (4), pulmonary artery banding (5) and systemic-to-pulmonary shunt (3). Ejection and shortening fraction (EF and SF), LV diastolic diameter and volume, RV and LV wall thickness, and RV/LV ratio were evaluated by echocardiography at referral, immediately before and one week after arterial switch.

Results. All children are alive and well, with a mean follow-up of 12 months (4-21 months). The values of $R V$ and $L V$ wall thickness, $\mathrm{RV} / \mathrm{LV}$ wall ratio, $\mathrm{LV}$ diastolic diameter and volume at referral, before and one week after arterial switch are reported in the Table. In all children LV function was adequate after arterial switch, with a mean $\mathrm{EF}=79.3 \%(66-87 \%)$ and a mean $\mathrm{SF}=41.7 \%(30-49 \%)$. Conclusions. Despite late referral, inadequate LV wall thickness and volume, children with TGA can undergo successful arterial switch, provided that an adequate LV preparation is performed through several surgical steps, with echocardiographic monitoring of the LV morphology and function.

\section{P283}

\section{Surgical treatment of primary cardiac tumors in} early infancy and childhood

M. Padalino, C. Basso, G. Sualuto Moreolo, V. Vida, O. Milanesi,

G. Thiene, D. Casarotto, G. Stellin

Institute of Pathological Anatonty, University of Padua, Padova, I

Objective: Primary heart tumors in infancy are rare. Despite their heterogenous nature and clinical features, surgical treatment is advocated when symptoms or hemodynamic impairment are present.

Materials and methods: From October 1985 to April 2000, seven children ( 3 males, 4 females, age ranging 5 days to 6.7 years, median 112 days) with a clinical diagnosis of cardiac mass were addressed to surgery. Diagnosis was made by two dimensional echocardiography and Doppler in all, while additional cardiac imaging was required in two patients.

Results: Complete surgical excision of the cardiac mass was possible in six patients, while orthotopic heart transplantation was performed in one. There were no surgical deaths. Microscopic study revealed fibroma in 2, myxoma in 2 , and hamartoma, multiple rhabdomyomas and teratoma in one each. One patient died due to cerebral neoplasm 3 years and 2 months after cardiac transplantation. At a mean follow up of 64.3 months (range 14-180 months), all the survivors are asymptomatic and well. There were no late reoperations. Two dimensional echocardiography showed good right and left ventricular function in all and no signs of residual or recurrent tumor.

Conclusion: Surgical excision of obstructive neoplastic masses in childhood is safely feasible. Cardiac transplantation is indicated when the tumor invades extensively the ventricular cavity and/or walls. While two-dimensional echocardiography remains a reliable diagnostic tool, a definite diagnosis of tumor histotype still deserves a thorough pathologic characterization.

\section{P284}

Ten year experience with mitral valve replacement in children under 5 years of age

$S$. Vobecky, A. Fournier

Hôpital Sainte Justine, Montréal, $C D N$

Objective: Mitral valve replacement (MVR) in children is a surgical challenge because it is often associated with other anomalies and reported morbidity and mortality are high

Methods: Retrospective review of children with MVR under 5 years of age during last ten years. Data are expressed as mean and standard deviations.

Results: Between January 1992 and July 200112 patients had a MVR at 3117 months (7-59) and at a weight of $10.63 .5 \mathrm{~kg}$ $(6.1-15.3)$ and a BSA of $0.490 .12 \mathrm{~m}(0.33-0.64)$. Mitral stenosis was the indication in 6 patients $(50 \%)$ and mitral insufficiency in the others. Among patients with stenosis, $3(50 \%)$ had a previous coarctation repair, one had an unsuccessful percutaneous balloon dilatation and 2 had no previous intervention; at MVR, 2 had a concomitant sub-aortic myomectomy. Among patients with insufficiency, one had an acute endocarditis with papillary muscle rupture and $5(83 \%)$ had previous intracardiac repair: 4 atrioventricular septal defect and one sub-aortic stenosis. Only $3(25 \%)$ patients were event free prior to MVR. The smallest valve implanted was a custom made $15 \mathrm{~mm}$. The CPB time was $121.546 .4 \mathrm{~min}$ $(72-249)$ and the ischemia time $68.719 .4 \mathrm{~min}(40-102)$. The follow-up was 38.622.4 months (13-88). There were no hospital deaths. One child with pulmonary hypertension eventually died after 26 months and one death was from leukaemia in a Down's syndrome patient after 45 months. One patient required a heart transplant for viral myocarditis 13 months post MVR. One patient had a supra annular scar tissue resection after 17 months and his valve was left in place. All patients were on oral anticoagulation with an INR $>3.5$ and no thrombo-embolic nor hemorragic event was recorded even though the patient with endocarditis had cerebral false aneurysms.

Conclusion: With a better survival in infants, it is important to achieve a MVR with a very low operative mortality in a young child contrary to reported data and valve related morbidity was low in our experience. A longer follow-up will answer the question of reoperation free interval and the next valve size that could be inserted.

\section{P285}

Simplified single patch technique for the repair of complete atrio-ventricular septal defect

D. Anil Kumar, R.N. Suresh Kumar, V.R. Pillai, C. G. Venkitachalam, Y.A. Nazer, T.B. Cartmill, I.M. Rao

Department of Cardiac Surgery, Abu Dhabi, UAE

We present our experience with the simplified single patch technique for the repair of complete atrio-ventricular septal defect 
avoiding the use of ventricular septal patch material. Between March 1998 and September 2001, fifteen infants underwent repair of complete atrio-ventricular septal defect with the use of this technique. The mean age at operation was six months (range 4 to 8 months) and the mean weight was $5.4 \mathrm{~kg}$ (range 4.1 to $7.7 \mathrm{~kg}$ ). Downs syndrome was present in eleven infants. Repair was done in all infants by direct suturing of the common atrio-ventricular valve leaflets to the crest of the ventricular septum, using interrupted $6-0$ gore tex sutures with pericardial pledgets placed on the right ventricular side. These were simultaneously passed through the leading edge of the pericardial patch that was used to close the defect in the artial septal component. In all cases, the cleft in the mitral component was repaired and no division of the valve leaflets was necessary. The technique was employed in all cases irrespective of the size of the defect in the ventricular septal component. There was no mortality; there were no pulmonary arterial hypertensive crises or heart block. The mean follow up was 13.2 months (range 2 to 42 months). One patient underwent mitral valve replacement after one year due to severe mitral regurgitation. The remaining fourteen patients had no significant residual ventricular septal defect or left ventricular outflow tract obstruction on echocardiography. Mitral valve regurgitation was assessed as mild in all the fourteen patients. In conclusion, repair of complete atrio-ventricular septal defect by direct suturing of the atrio-ventricular valve leaflets to the crest of the ventricular septum by this technique simplifies the repair, minimises ischemic time, is easily reproducible and less time consuming. It does not interfere with valve function and does not cause left ventricular outflow tract obstruction. The early results are encouraging.

\section{P286}

Long-term results of a modified repair technique in Ebstein's anomaly

N. Nagdyman, P. Ewert, E. Riesenkampff, R. Hetzer, P.E. Lange

Deutsches Herzzentrum Berlin, Berlin, $D$

Objective: Surgical procedure in Ebstein's malformation is done by a modified repair technique at our institution. This technique restructures the valve mechanism at the level of the true tricuspid anulus by using the most mobile leaflet for valve closure without plication of the atrialized chamber. We present our long-term results of this reconstruction technique.

Methods: Between October 1988 and February 2000, tricuspid valve repair was performed in 22 patients (pts) with Ebstein's anomaly ( 15 female, 7 male; 2 to 55 years, median 17 years). Indication for operation was congestive heart failure of various degree, and tricuspid incompetence with grade II ( $2 \mathrm{pts})$, grade III (15 pts) and grade III (5 pts). Additional congenital malformations were repaired simultaneously. Follow-up period was 9 years (range 1.5 to 13 years). All patients were followed up at least once a year. Results: We observed no operative death, one pt died of recurrent sepsis 2 months after the operation, another patient died of congestive heart failure within two weeks after the operation. One case of late death occured three years after the operation due to severe mitral valve insufficiency and congestive heart failure.

New York Heart Association functional class improved from preoperatively 3.1 to postoperatively 1.7 ( $p<0.001$ ). Tricuspid valve incompetence decreased from a mean grade of 3.1 to 1.7 $(\mathrm{p}<0.001)$. No aneurysm of the former atrialized right ventricle was observed. Systemic arterial saturation improved significantly (91\% to $98 \%, p<0.001)$.

Conclusion: These long-term results demonstrate good clinical outcome, even in cases where tricuspid valve replacement could have been discussed.

\section{P287}

Early and late outcome after different modifications of the Fontan procedure

R.L.E. van Loon, M. Dalinghaus, A.J.J.C. Bogers, R.M.F. Berger

Sophia Children's Hospital, Rotterdam, The Netherlands, Rotterdam, NL

Background: Early ventricular volume unloading, through staging of the Fontan procedure in patients with a single ventricle, has been suggested to be beneficial to outcome.

Objectives: Assessment of early and late outcome of pediatric patients who underwent different modifications of the Fontan procedure

Methods and results: We retrospectively reviewed the clinical records of 114 consecutive pediatric patients who underwent a Fontan procedure at our center in the period 1978 to 2001 Median length of follow-up was 5.4 years (range 0 to 22.8 years). Median age at follow-up was 7.3 years (range 0.4 to 26.8 years). Male : female ratio was $2: 1$. Diagnoses included tricuspid atresia $(n=25)$, double inlet left ventricle $(n=25)$, pulmonary atresia with intact septum $(\mathrm{n}=10)$, hypoplastic left heart syndrome $(\mathrm{n}=9)$ and other complex univentricular heart malformations $(\mathrm{n}=45)$. Surgical procedures prior to the Fontan procedure were performed in $83 / 114$ patients $(73 \%)$. Three modifications of the Fontan procedure were utilized: atriopulmonary connection $(\mathrm{n}=30)$, single-stage total cavopulmonary connection $(\mathrm{n}=21)$ and staged total cavopulmonary connection $(n=63)$. Of the last mentioned patients 45 had completed their staged total cavopulmonary connection. The surgical approach evolved during the study period, from single staged procedures early in the experience, to staged later in the experience. Median age at completion of the Fontan procedure was 3.2 years (range 0.4 to 17 years).

Thirty-four of 114 patients (30\%) died. Of these deaths 53\% $(\mathrm{n}=18)$ occurred within 30 days after surgery. No mortality was seen from nine years postoperatively. In a representative subset of patients 51/56 (91\%) were in NYHA I/II at a median follow-up of 5.8 years. Mortality was associated with the type of surgical procedure: lowest mortality occurred after the staged Fontan procedure (see graph). Younger age at single staged procedures was associated with increased early mortality. Univariate risk factor analysis indicated that cardiac diagnosis and ventricular morphology were not correlated with mortality.

Conclusions: In our experience, staging of the Fontan procedure had a better outcome compared to non-staged procedures. In the total patient group, mortality occurred mainly in the early postoperative period. Surviving patients in general were in good functional condition.

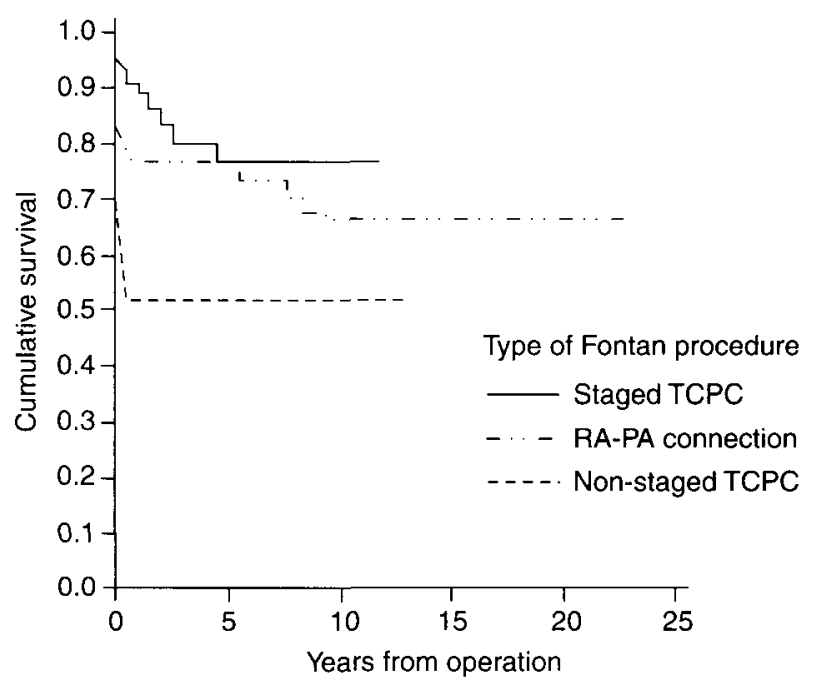




\section{P288}

Minimal invasive approaches in pediatric cardiac surgery

A.R. Tiete, J.S. Sachweh, R. Kozlik-Feldmann, H. Netz,

B. Reichart, S.H. Daebritz

LMU München, Klinikum Grosshadern, Dept. of Cardiac Surgery, Munich, $D$

Objective: Full midline sternotomy is the standard approach for the correction of congenital heart defects. However, minimallyinvasive approaches will more and more be favoured in special cases with regards to better cosmetic results.

Methods: Between July 1999 and April 2001, 25 pediatric patients (9 male, 16 female) were operated on using minimally invasive approaches. Indications for surgery were: atrial septal defect ( $\mathrm{n}=19,2$ with partial anomalous pulmonary venous connection), ventricular septal defect $(n=2)$, ostium primum defect $(n=2)$, intermediate-type atrioventricular canal $(\mathrm{n}=1)$ and Tetralogy of Fallot $(n=1)$. Mean age was 5.3 years (range 2.1 months -14.8 years), mean weight was $19.6 \mathrm{~kg}$ (range $4.2-63 \mathrm{~kg}$ ). In female patients with any kind of atrial septal defect we preferred a right limited anterolateral thoracotomy. A ministernotomy was performed in male patients, in patients under 6 months of age, and for the repair of malformations other than atrial septal defects. All patients were cannulated via the thoracotomy or ministernotomy without additional incisions particularly in the groin.

Results: There were no deaths and no perioperative complications. Mean operation time was $3.23 \mathrm{~h}$ (range $2-5.15 \mathrm{~h}$ ). Twelve patients were extubated immediatly after surgery, mean ventilation time in the other 13 patients was $12.1 \mathrm{~h}$ (range 2-7h). Mean ICU-stay was 1.50 .75 days (range 1-4 days), mean hospitalisation was 8.32 .2 days (range 5-14 days). The only postoperative complication was a pneumothorax. Follow- up with a mean of 4.84 .6 months was uneventful.

Conclusions: Intracardiac repair for some congenital heart defects can safely and efficaciously be performed via minimally-invasive approaches. Indications are expanding towards more complex defects. Exposure for precise repair is good and additional incisions for cannulation can be avoided. The cosmetic results are excellent.

\section{P289}

Intraoperative stenting: advantages of a combined surgical and catheter interventional approach R. Bokenkamp, N.A. Blom, D. De Wolf, K. Francois, M. G. Hazenkamp Leiden University Medical Center, Leiden, NL

Stenting of stenotic pulmonary arteries has become an accepted catheter intervention in children. Intraoperative stenting can be an attractive alternative in patients requiring cardiac surgery, in infants or in those with limited vascular access.

Methods: Intraoperative stenting was performed in six children. All patients (pts) had stenosed pulmonary arteries and had undergone previous cardiac surgery. Median age was 12 months (range 1 week-12 yrs), median weight was $8 \mathrm{~kg}$ (range 2,1-20 kg). In 4 pts with PA/VSD stenting was combined with biventricular repair or unifocalisation. In one pt $(2.1 \mathrm{~kg})$ intraoperative stenting was performed to relief severe bilateral branch obstruction in the early postoperative phase after truncus repair. In one pt, a year after operation of IAA type B, stenting was combined with retrieval of a dislodged interventionally placed stent. Stents were placed under fluoroscopy $(\mathrm{n}=1)$ or direct surgical vision $(\mathrm{n}=5)$ while patients were on CBP. The paediatric cardiologist performed stent selection and inflation. Palmaz-Schatz and coronary artery stents were used. Balloon size ranged from 4 to $8 \mathrm{~mm}$. All patients received antiplatelet therapy.
Results: 10 stents were placed successfully. There was no early mortality and the postoperative course was uncomplicated. All patients clinically improved after the operation. One pt with PA/VSD died 8 month later during biventricular repair. Patency of the stents was demonstrated either by angiography or echocardiography. Redilatation was necessary twice in one pt. Follow-up ranged from 2 month to $5 \mathrm{yrs}$.

Conclusion: Intraoperative stenting of the pulmonary arteries is a fast and highly effective technique. It combines the advantages of an interventional technique and direct surgical exposure.

\section{P290}

Surgical outcomes in 74 patients with atrial isomerism

B.H.P. Nagel, J. Paul, D. Barron, J. G. C. W'right, J. Giovanni,

W.J. Brawn, O. Stumper

University Children's Hospital, Essen, D

Over a 15 year period 74 patients with atrial isomerism underwent surgery at our institution. The spectrum of complex cardiac and extracardiac anomalies, the surgical results and the causes for late mortality were analysed.

All patients with either left (LAI) or right (RAI) atrial isomerism were identified from a surgical cardiac database between 1985 and 2000. Data was analysed from casenotes, catheter reports, operation notes and from echo and angiography recordings.

37 patients had LAI and 37 patients had RAI. Cardiac defects included interrupted IVC $(86 \%$ in LAI vs. $0 \%$ in RAI), anomalous pulmonary venous drainage ( $27 \%$ vs. $85 \%)$, obstructed pulmonary veins (OPV) $(0 \%$ vs. $45 \%)$, single ventricle physiology $(54 \%$ vs. $81 \%)$, common atrioventricular valve ( $89 \%$ vs. $86 \%$ ), ventriculoarterial discordance $(30 \%$ vs. $77 \%$ ), pulmonary outflow obstruction (POO) (41\% vs. $86 \%$ ). Extracardiac defects were encountered in $30 \%$ vs. $19 \%$ of patients. Overall surgical mortality rate in LAI was $24 \%$ ( $18 \%$ for biventricular vs. $30 \%$ for single ventricle repair), in RAI $38 \%(50 \%$ vs. $35 \%)(p<0.05)$. Mean age at first intervention was 0.9 y. (range $0.01-13.8$ ) in LAI and 0.5 y. $(0-4.8)$ in RAI (n.s.); mean age at death was $3.9 \mathrm{y} .(0.01-13.9)$ and $1.6 \mathrm{y} .(0.01-7.1)$ (n.s.). Causes for late mortality ( $5 / 9$ in LAI and $8 / 14$ in RAI) were sepsis $(n=4)$, heart failure due to severe AV valve regurgitation $(n=2)$, failing cavopulmonary shunt or Fontan $(\mathrm{n}=2)$, persistent pulmonary hypertension due to recurrent OPV $(n=4)$ and sudden death of unknown cause $(n=1)$. Of the patients with obstructed TAPVD $(n=10)$ all 6 patients requiring BT shunt for POO died. Of the 4 patients without significant POO only 2 survived. Overall survival at $1,5,10$ and 15 years were $86 \%, 83 \%, 78 \%$ and $51 \%$ in LAI and $78 \%, 64 \%, 58 \%$ and $58 \%$ in RAI. Survival rate at 15 years did not differ significantly between the groups. The only independent risk factor for mortality was obstructed pulmonary veins. Conclusions: Surgical long term outcome of infants and children with LAI and RAI do nor differ and remain unfavourable. Pulmonary venous obstruction in RAI is a serious problem. Major causes for late mortality are sepsis and recurrent pulmonary vein obstruction.

\section{P291}

Outcome and right ventricular growth potential after surgical decompression for pulmonary atresia with intact ventricular septum

B. Ganev, G. Christov, S. Lazarov, V. Pilossoff

National Heart Hospital, Sofia, BG

Objective: To perform a retrospective analysis of the outcome after right ventricular decompression with transannular patch (RVD-TAP) for pulmonary atresia with intact ventricular septum 
(PA/IVS); to evaluate right ventricle growth and its relation to reaching definite two-ventricle circulation.

Patients and methods: Nineteen consecutive patients (pts) with PA/IVS were operated on between 1988 and 1998. Eight of them underwent RVD-TAP and are included in the study. The median age at operation was 15 days (4-58). Two pts had previous procedures: one had unsuccessful transcatheter balloon valvotomy and another - ineffective surgical valvotomy. Two pts received concomitant/subsequent systemic-to-pulmonary artery (SP) shunt. In the rest $6 / 8$ pts PDA was maintained postoperatively with intravenous PgE1 for median 7, 5 days (3-21). Patients who survived the early postoperative period were followed up at about $1,6,12$ mo and yearly thereafter for median 62,5 months (45-90). Tricuspid valve (TV) Z-value is calculated retrospectively on the basis of echocardiography measurements. Two-ventricle repair is defined as absence of residual RV outflow tract obstuction and intra- or extracardiac shunts plus pulmonary circulation entirely provided by RV.

Results: Four of the patients died in the early postoperative period 3 pts on 1-st postoperative day with low cardiac output and 1pt on 30-th postoperative day with sepsis. The patient with previous balloon pulmonary valvotomy and both pts with SP shunt are among non-survivors. There are no late deaths. Preoperative TV Z-value is $=-2,0$. There is no statistically significant difference in the TV $Z$-value between survivors and non-survivors. The absolute TV dimensions increased with age but TV Z-value tends to decrease. All survivors did not have residual RVOT obstruction and reached twoventricle circulation with spontaneous $\mathrm{PFO}$ closure after median 30 mo (7-75) and PDA closure after median 1,85 mo $\langle 0,5-20)$. Conclusions: RVD-TAP provides long term survival for PA/IVS with normal or near normal RV despite the still high early postoperative mortality. RV growth rate is lower than body growth rate. Despite that two ventricle circulation in cases without surgical SP shunt can be achieved with spontaneous closure of PDA and PFO. Absence of residual RVOT obstruction is an obligatory condition.

\section{P292}

Surgical repair of mitral valve anomalies in pediatric age R. M. Bini, A. Giamberti, G. Pom, G.F. Butera, M. Chessa,

E. Mazza, A. Cazzaniga, N. Carano, A. Agnetti, M. Carminati,

A. Frigiola, U. Squarcia

Istituto Policlinico San Donato, S. Donato Milanese (MI), I

Between January 1990 and December 2001, 35 patients (1 mo-14 yrs, mean $4.5 \mathrm{yrs}$ ), were referred to our center for treatment of MV anomalies. Of these, 12 pts had residual MV dysfunction after correction of A-V canal and 23 had primitive congenital MV disease. Ten pts had MV insufficiency ( $6 \mathrm{MV}$ prolapse, 3 dysplastic leaflet and annulus dilation, 1 isolated cleft of anterior leaflet). Thirteen pts had MV stenosis ( 5 parachute valve, 6 dysplastic valves, 2 supravalvar rings). Associated cardiac defects were present in 13 cases.

Procedures: Mitral insufficiency (10 pts): suture of cleft 1, Alfieri technique 2, Annuloplasty 3, Annuloplasty and other manoeuvres on chordae and papillary muscles 4. Mitral stenosis (13): Resection of supravalvar rings 2, Splitting of papillary muscles and commissurotomy 5, Commissurotomy and fenestration of chordae and/or splitting of papillaty muscles 3, Ross-Kabbani procedure 1, Mitral valve replacement 2

Results: There were $3(13 \%)$ post operative deaths: 3 early deaths, one because of residual severe MV stenosis and low cardiac output and one for intestinal hemorrage; one $5 \mathrm{wks}$ p.o. due to neurological complication following acute LV failure.
Follow-Up: Twenty pts were available for F/U at $2-107$ mos (mean 38 mos). Of these 14 are in NYHA functional class 1,3 in class 2 and 2 in class 3 . One pt required MV replacement 1 yr p.o. The echocardiograms shows mild MV regurgitation in 12 pts, moderate regurgitation in 4 and moderate stenosis and regurgitation in $1 \mathrm{pt}$. Two pts with valve replacement received a mechanical prostheses and are under oral anticoagulation. Freedom from reintervention is 0.85 after the first yr and remain stable thereafter Conclusion: Different surgical techniques are available in congenital MV insufficiency and stenosis. Newer techniques such as the Ross-Kabbani procedure could represent the best option in selected cases, in order to avoid mechanical valves and to allow tissue growth.

\section{P293}

Outcome after two-coronary repair of anomalous left coronary artery from the pulmonary artery without mitral valve repair

G. Christov, Ts. Loukanov, S. Lazarov, P. Mitev, I. Velkovski, A. Lacheva, V. Pilossoff

National Heart Hospital, Sofia, BG

Objective: To perform a single centre retrospective study of the outcome after two-coronary repair of anomalous left coronary artery from the pulmonary artery (ALCAPA).

Patients \& methods: All ten consecutive patients (pts) with ALCAPA operated on between 1987 and 2001 are included in the study. Median age at diagnosis was 10,5 mo $(3,3-72)$, six being under $1 \mathrm{y}$. The median interval between diagnosis and operation was 2,0 mo $(0,1-14,0)$. Median age at operation was $16,5 \mathrm{mo}$ (4-84). Median follow-up period is $3,9 \mathrm{y}(0,9-13,8)$ to the median age of $7,0 y(1,7-16,1)$. Diagnosis was made by echocardiography and confirmed by angiography. Eight pts had congestive heart failure. Nine pts had depressed ejection fraction (EF) and left ventricle (LV) dilatation. Eight pts had mitral regurgitation (MR) severe in 2 , moderate in 4 , mild or trivial in 2 . All pts had ECG changes both deep Q and ST-T type in 5, only deep Q type in 3 and only ST-T type in 2 . All pts underwent two-coronary repair - direct aortic implantation in 6pts and intrapulmonary baffle (Takeuchi) repair in 4 pts. None received mitral valve (MV) repair.

Results: One patient could not be weaned from cardiopulmonary bypass after direct aortic implantation and died of LCA ostial stenosis with acute myocardial ischemia. Median ICU stay was $10 \mathrm{~d}$ (6-17). Median mechanical ventilation was $72 \mathrm{~h}(20-264)$. Till 30th postoperative day EF did not change significantly; LV diameter decreased significantly with normalization in 5pts; MR regressed - none had moderate or severe MR, 3 had mild, 3 trivia MR; 3 of 4 pts with Takeuchi repair had mild supravalvar pulmonary stenosis. At the end of follow-up all have normal functional capacity, all except 2 have normal EF (respectively 60 and 61\%); all have normal LV diameter; $1 \mathrm{pt}$ has mild, 5 trivial MR: supravalvar pulmonary stenosis remains mild; 3 pts have ECG changes of ST-T type. All 9 survivors are free from reoperations. Conclusion: This confirms very good short-term and long-term results of two-coronary repair of ALCAPA without MV repair In our cases MR regressed rapidly postoperatively.

P294

Anatomical repair of complete atrioventricular septal defect

Hani K. Najm, MD, Msc, Michael Godman MD

King Abdulaziz Cardiac Center, Riyadh, SAU

Background: A better understanding of the morphology of complete atrioventricular septal defects (CAVSD) has impacted on 
surgical techniques and results. Competency of the left atrioventricular valve plays an important role in the outcome of repair of these defects. On some occasions the leaflet tissue is deficient and repair becomes difficult. We present a modified technique for the repair of this defect.

Material and method: This technique was performed on 16 infants with CAVSD at a mean age of 5 months. The procedure is performed on standard cardiopulmonary bypass with cardioplegic arrest. The right atrium is opened and the anatomy is evaluated. Both superior and inferior leaflets are divided routinely to completely expose the inlet VSD. An autologous pericardial patch, sized precisely, is sutured to the right side of the ventricular septum leaving 3-4 $\mathrm{mm}$ of extra patch beyond the plane of the annulus to augment the divided leaflet tissue of the left $A-V$ valve. The cleft is closed. A second autologous pericardial atrial septal patch is attached on the body of the VSD patch apical to the plane of the annulus, thereby allowing 3-4 mm of the VSD patch to augment the left $\mathrm{AV}$ valve. The remainer of the patch is sutured to the edge of the atrial septal defect.

Results: There were no deaths among these infants. At postoperative echo there was no significant postoperative left $A V$ valve regurgitation, residual VSD's or left ventricular outflow tract obstruction. The contribution of the patch-augmented left AV valve to competency is clearly seen. The right AV valve is apically offset as in normal subjects.

Conclusions: The use of this modified technique yields good anatomical repair by providing good exposure and by allowing reconstruction of both AV valves independent of the other. This technique is in particular helpful in cases of deficient left $\mathrm{AV}$ valve tissue.

\section{P295}

Inhaled nitric oxide (INO) in association with ventricular assist device (VAD) in paediatric patients with cardiogenic shock after cardiac surgery

E. Mazza, A. Ballotta, C. Panzeri

Istituto Policlinico San Donato, S. Donato Milanese (MI), I

Purpose: After surgical repair of congenital heart defects in infants very severe cardiac failure can occur with a refractory low cardiac output state requiring some form of extracorporeal life support. In our experience the best results are with univentricular device and when the failure is biventricular we try to assist left ventricle with a VAD and the right with INO. We describe 4 cases in which we have associated INO and VAD for cardiogenic shock after cardiac surgery.

Methods: We descibe 3 cases. 1) First case: tetralogy of Fallot of six months of age with perioperative severe left ventricular failure and moderate RV failure with the impossibility of weaning from CPB. We support the left ventricle with LVAD (centrifugal pump) and RV with INO (15 $5 \mathrm{ppm})$. 2) Second case: patients of 5 years of age with destrocardia, ventricular septal defect and severe mitral regurgitation with biventricular heart failure because of postoperative myocardial infarction refractory to any medical treatment. The supporting system consisted in LVAD and INO. 3) Third case: partial atrioventricular septal defect of 4 years of age with the same problems of the third (postoperative myocardial infarction). LVAD and INO were the support system.

Results: the duration of VAD therapy was 3 days in the first case, 4 days in the second and the third. The INO therapy was stopped 2 days after VAD removal in all the cases. All the 3 patients have been discharged. In a early follow-up they are alive and in good shape.
Conclusion: Peri- and postoperative heart failure is often biventricular, however taking the patient in LVAD, right ventricle might be supported reducing the pulmonary vascular resistance and reducing the afterload and improving the performance with iNO that increase LVAD flow index as well: this could be a strong point in the decision of implanting monoventricular instead of a biventricular (or ECMO) supporting circulatory device.

\section{P296}

Evolution of blood lactate levels after cardiac surgery in infants and neonates - correlations with cardiovascular risk

J. Fragata, G. Nogueira, P. Coelho, N. Banazol, M. Primo, A. Ferro,

N.D. Silva, I. Fragata, J. Roquette

Hospital de Santa Marta, Lishoa, $P$

Background: High lactate levels have been associated with increased morbidity and mortality in different forms of shock and, recently, were shown to correlate with mortality after cardiac surgery in infancy.

Objectives: Establish the evolution pattern of blood lactate levels at different times during the post-operative period of cardiac surgery in neonates and infants and to correlate those levels with both mortality and cardiovascular morbidity.

Methods: We retrospectively studied 51 pts with a mean age of 6,2 months ( 3 days -24 months) including 18 neonates $5,1-2,5 \mathrm{Kg}$ weight, operated under extracorporeal circulation and hypother$\mathrm{mia}$, by the same team. Lactate levels $(\mathrm{mg} / \mathrm{d}$ ) were evaluated on admission to the ITU and at $6,12,24$ hours post-op. Blood lactate measured at $6 \mathrm{hr}$ post-op was correlated with blood $\mathrm{pH}$ and Bicarbonat, as well as with the combined inotrope dose (Dopamine + Dobutamine) in $\mathrm{mcg} / \mathrm{Kg} / \mathrm{m}$ at the same time. Every morbidity and mortality event was registered and correlated to the maximum lactate level.

Results: Lactate levels dropped steadily from $51,3-36,8 \mathrm{mg} / \mathrm{dl}$, on ICU admission, to $22,8-31.9 \mathrm{mg} / \mathrm{dl}$ at $24 \mathrm{~h} \mathrm{PO}(\mathrm{p}=0,004)$ in the 45 pts with no morbidity or mortality events. Lactate levels above $100 \mathrm{mg} / \mathrm{dl}$, at any time $(\mathrm{n}=6)$, were associated with at least one adverse post-operative event - low cardiac output, bleeding/tamponade or cardiac arrest and in three pts that died lactate levels reached $150 \mathrm{mg} / \mathrm{dl}$ and over. Correlation of lactate levels with $\mathrm{pH}$ and bicarbonate blood levels was weak, but its correlation with the total dose of inotopic drugs at $6 \mathrm{~h}$ post-operatively was significant: $\mathrm{r}=0,52(\mathrm{p}=0,005)$.

Conclusions: Increased blood lactate levels after open heart surgery in infants are associated with higher morbidity and mortality rates. Fluctuations in lactate levels followed the hemodynamic course closely and seemed more sensitive to access tissue hypoperfusion than traditionally used markers. Unexpected upward variations, even in the absence of other signs, should be taken as a serious indicator and prompt to immediate diagnostic or therapeutic action.

\section{P297}

Right ventricular to pulmonary artery shunt instead of modified Blalock Taussig shunt improves diastolic systemic and cerebral flow in newborns after the Norwood procedure

G. Tulzer, R Mair, R. Gitter, E Lechner, E Sames, G Geiselseder Children's Heart Center Linz, Linz, A

In newborns, who underwent a Norwood procedure due to a functionally univentricular heart combined with aortic arch hypoplasia, pulmonary blood flow is usually established via a 
modified Blalock Taussig shunt (BTS). The lower pulmonary vascular resistance causes a diastolic run-off into the pulmonary vascular bed, leading to low diastolic systemic pressures and thus decreased coronary perfusion pressure. Especially with large shunts, the single ventricle faces a decreasing coronary perfusion with increasing workload. This has been attributed to contribute to the early and late mortality and morbidity after stage I palliation. Placement of a right ventricular to pulmonary shunt (RV/PA) avoids this diastolic run-off and should therefore improve systemic diastolic flow. To assess differences in hemodynamics echocardiographic data of 16 newborns, who underwent a Norwood procedures was analyzed. 8 newborns with a BTS were compared to 8 newborns with RV/PA Shunt ( $5 \mathrm{~mm}$ diameter). Between the groups there was no significant difference in weight, preoperative complications or bypass time. There was no significant $A V$ valve or pulmonary valve regurgitation except in 1 case with $\mathrm{RV} / \mathrm{PA}$ Shunt. Echocardiography was carried out, when children were extubated and in a hemodynamically stable condition. There were no significant differences in ventricular inflow and outflow velocities or in shortening fraction. Doppler examination of descending aortic flow showed holodiastolic reversal of flow in all children with BTS but holodiastolic antegrade flow in $7 / 8$ children with RV/PA Shunt. Doppler interrogation of the arteria cerebri anteror showed significantly lower pulsatility index due to higher enddiastolic and mean velocities in the group with RV/PA Shunts. Measured gradients across the RV/PA Shunt were $3,46 \pm 0,7 \mathrm{~m} / \mathrm{s}$ (mean \pm std dev) suggesting normal pulmonary artery pressures. Right ventricular to pulmonary artery shunt instead of modified Blalock Taussig shunt improves diastolic systemic and cerebral flow in newborns after the Norwood procedure and should thus improve coronary and brain perfusion leading to a more stable hemodynamic situation after stage I palliation.

\section{P298}

Takayasu's arteritis in children: short and midterm outcome after surgical and interventional procedures A. Buerge, B. Faidutti, B. Friedli, D. Didier, M. Beghetti, A. Kalangos, E. Jaeggi

Pediatric Cardiology, University Children's Hospital, Geneva, $\mathrm{CH}$

There is little information on reconstructive arterial surgery for Takayasu's disease (TI) in children. Since 1989, 9 children
(5 male, 4 female; mean: $12.7+2.6$ years of age) with TD were referred to our center. Baseline cardio-vascular assessment by angiography $(n=7)$ or angio-IRM $(n=6)$ were performed in all: in 2 patients the disease was confined to the thoracic part of the aorta $(\mathrm{T})$, in 4 to the abdominal aorta (A), and 3 had combined thoraco-abdominal aortic disease (TA). In addition, between 2 to 7 medium-sized branch arteries were affected: stenotic lesions predominated, and included complete occlusion of abdominal arteries in 3 patients. Seven children underwent complex surgical procedures, 1 had balloon dilatation of renal and mesenteric arteries, and 1 a combined surgical-interventional approach. Both T patients underwent homograft replacement of the aortic arch or the ascending aorta. Of the 6 operated A and TA cases, 2 underwent thoraco-abdominal bypass surgery with a Goretex tube, 4 had abdominal aorta replacement with a Dacron (3) or homograft, and 5 had interposition grafts for various peripheral stenosis. There were no peri-operative deaths. The arterial hypertension resolved in all and the cardiac function normalized in 2 of 3 cases with dilative cardiomyopathy prior to surgery. However, 3 months to 12 years after intervention, $1 \mathrm{~T}$ patient had died suddenly and 2 other had non-fatal disease progression, of whom 1 required surgical re-intervention.

Conclusion. All patients in our series were amenable to surgery or catheter intervention, with $0 \%$ perioperative mortality and a satisfactory short and mid-term outcome.

P299 (see Abstract 26)

Serious complications after implantation of SynerGraft valved conduits in the pulmonary position

U. Salzer-Muhar, E. Kitzmiler, M. Marx, M. Schlemmer, P. Sinon, G. Weigel, G. Amann

Division of Pediatric Cardiology, Department of Pediatrics, Vienna, $A$ 